\title{
ECOLOGICAL STUDIES RELATED TO THE CONSTRUCTION OF THE DEFENSE WASTE PROCESSING FACILITY \\ ON THE SAVANIAH RIVER SITE
}

FY-1993 AMNUAL REPORT

\author{
Division of Wildife Ecology and Toxicology \\ Savannah River Ecology Laboratory \\ University of Georgia \\ Drawex E \\ Aiken, SC 29802
}

November 1994

Program Coordinator and SREL Contact: Laura I. Janecek

DE-AC09-76SR00-819 
TABLE OF CONTENTS

I. INTRODUCTION AND OVERVIEW...................... 1

LITERATURE CITED........................... 4

II. WATER QUALITY MONITORING OF PERIPHERAL STREAMS

INTRODUCTION............................. 6

METHODS.................................. 6

site selection........................ 6

sampling........................... 8

Data analysis........................... 9

RESULTS AND DISCUSSION....................... 13

Introduction. ......................... 13

McQueen Branch.......................... 15

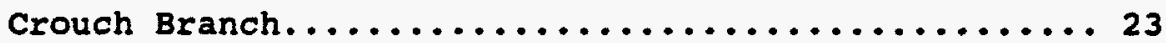

Upper Three Runs Creek.................... 31

SUMMARY................................. 39

CONCLUSION................................. 40

LITERATURE CITED.......................... 41

APPENDIX A (DWPF WATER QUALITY DATA--1983 TO 1993)....... 43

APPENDIX B (SUMMARY OF STATISTICAL TESTS) $\ldots \ldots \ldots \ldots \ldots \ldots \ldots 84$

APPENDIX C (SUMMARY FOR EACH CONSTRUCTION PERIOD) ........ 86

III. AMPHIBIAN USE OF BREEDING SITES CREATED AS EXPERIMENTAL MITIGATION FOR WETLAND LOSS...................... 96

INTRODUCTION.............................. 96

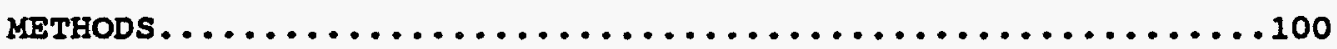

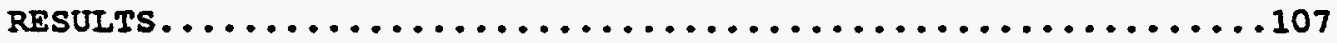

Discussion..................................... 113

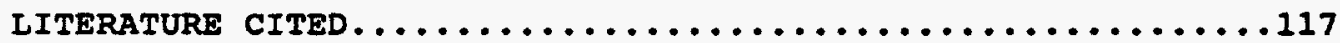




\section{DISCLAIMER}

This report was prepared as an account of work sponsored by an agency of the United States Government. Neither the United States Government nor any agency thereof, nor any of their employees, make any warranty, express or implied, or assumes any legal liability or responsibility for the accuracy, completeness, or usefunness of any information, apparatus, product, or process disclosed, or represents that its use would not infringe privately owned rights. Reference herein to any specific commercial product, process, or service by trade name, trademark, manufacturer, or otherwise does not necessarily constitute or imply its endorsement, recommendation, or favoring by the United States Government or any agency thereof. The views and opinions of authors expressed herein do not necessarily state or reflect those of the United States Government or any agency thereof. 


\section{DISClamMER}

Portions of this document may be illegible in electronic image products. Images are produced from the best available original document. 


\section{FOREWARD}

Construction of the Defense Waste Processing Facility (DWPF) on the Savannah River Site (SRS) began during FY-1984. The Savannah River Ecology Laboratory (SREL) has completed 15 years of ecological studies related to the construction of the DWPF complex. Prior to construction, the 600-acre site (S-Area) contained a Carolina bay and the headwaters of a stream. Research conducted by the SREL has focused primarily on four questions related to these wetlands: 1) Prior to construction, what fauna and flora were present at the DWPF site and at similar, yet undisturbed, alternative sites? 2) By comparing the Carolina bay at the DWPF site (Sun Bay) with an undisturbed control Carolina bay (Rainbow Bay), what effect is construction having on the organisms that inhabited the DWPF site? 3) By comparing control streams with streams on the periphery of the DWPF site, what effect is construction having on the peripheral streams? 4) How effective have efforts been to lessen the impacts of construction, both with respect to erosion control measures and the construction of "refuge ponds" as alternative breeding sites for amphibians that formerly bred at sun Bay?

Through the long-term census taking of biota at the DWPF site and Rainbow Bay, SREL has been evaluating the impact of construction on the biota and the effectiveness of mitigation efforts. Similarly, the effects of erosion from the DWPF site on the water quality of S-Area peripheral streams are being assessed. This research provides supporting data relevant to the National Environmental Policy Act (NEPA) of 1969, the Endangered species Act of 1973, Executive Orders 11988 (Floodplain Management) and 11990 (Protection of Wetlands), and United States Department of Energy (DOE) Guidelines for Compliance with Floodplain/Wetland Environmental Review Requirements (10 CFR $1022)$. 


\section{INTRODUCTION AND OVERVIEW}

David E. Scott and Joseph H.K. Pechmann

The Savannah River Ecology Laboratory initiated ecological studies related to the construction of the DWPF on the SRS in FY-1979. Two areas have been used for biological surveys and long-term monitoring: the DWPF construction site (S-Area and $\mathrm{z}$-Area), and two control sites (Rainbow Bay and Tinker Creek). The Rainbow Bay study area and $s$-Area are located within $5 \mathrm{~km}$ of each other on the SRS (Fig. I-1), and both once contained Carolina bays which were very similar ecologically (SREL 1980). One goal of the SREL's faunal studies is to compare the natural variation in amphibian populations at the Rainbow Bay control site to the variation observed at the human-altered site (Sun Bay, formerly on the DWPF construction site). Amphibian populations exhibit large year-to-year variation in population size and breeding success, thus long-term studies are necessary to separate natural variation from variation due to human perturbations (Pechmann et al. 1991, 1993; Vitt 1981, Vitt et al. 1982).

Pre-construction biological surveys included data on vegetation, birds, mammals, amphibians, reptiles, fish and several invertebrate groups (SREL 1979, 1980). No species on the Federal Endangered or Threatened lists were found on either site, but several plants and animals of threatened or specialconcern status in South Carolina were present (SREL 1980, Vitt 1981) and the gopher frog (Rana areolata) is currently being considered for federal listing. DWPF construction began in FY-1984. Continuing studies are directed towards assessing construction impacts on the biota, and towards modeling the effects of alteration of wetland hydroperiod on the biota. Primary emphasis is being placed on evaluating the effectiveness of mitigation measures undertaken by the DOE. Also, a special automated audio-monitoring technique is being tested at Rainbow Bay (and other SRS habitats). This technique will greatly enhance site assessment for amphibians. 


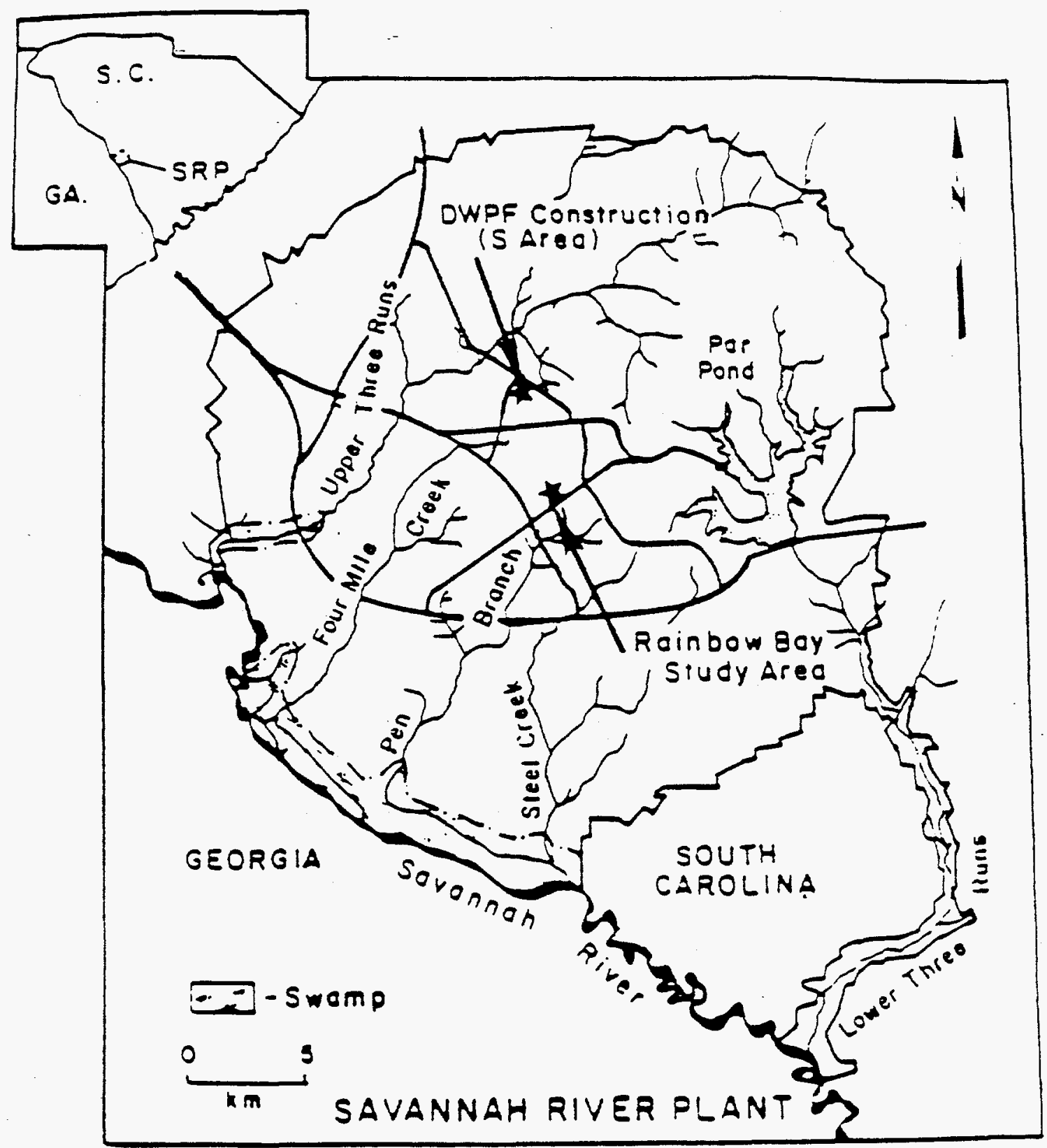

Figure I-1 Locations of the DWPF construction site (S-Area) and the control site (Rainbow Bay) on the Savannah River Site. 
SREL began baseline water quality monitoring on $s$-Area peripheral streams in November 1982 (prior to construction) to quantify natural variation in water quality parameters. Sampling has continued to the present on the streams that drain the DWPF site (Upper Three Runs Creek, McQueen Branch, and Crouch Branch; Figure II-1) and on a nearby, unimpacted blackwater stream, Tinker Creek. Erosion resulting from DWPF construction potentially could affect the productivity and biotic diversity of McQueen Branch, Crouch Branch and Upper Three Runs Creek (U.S. DOE 1982). Results of a baseline survey of macroinvertebrates in these streams were reported in Pechmann at al. (1984). Chapter II of this report contains the FY-1993 water quality results which were obtained during the continuing DWPF construction.

In FY-1984, the DWPF construction eliminated sun Bay in S-Area. Carolina bays are productive, natural wetlands which serve as important breeding sites for many species of amphibians (Bennett et al. 1979, Gibbons and Semlitsch 1981, Sharitz and Gibbons 1982). Amphibians are the most prevalent group of vertebrates on both the Rainbow Bay control site and the DWPF site (SREL 1980). A major objective of the SREL studies has been to evaluate the effects of the 1088 of Sun Bay on the breeding success of amphibians in S-Area (Pechmann et al. 1985, scott et al. 1986). In an experimental attempt to mitigate the loss of the natural breeding habitat in S-Area (i.e., Sun Bay), four refuge ponds were constructed. Only three of these are currently in operation, because of the loss of one due to unanticipated construction activities. A summary of refuge pond captures is presented in Chapter III. 


\section{IITERATURE CITED}

Bennett, S.H., J.W. Gibbons and J. Glanville. 1979. Terrestrial activity, abundance and diversity of amphibians in differently managed forest types. Am. Midl. Nat. 103:412-416.

Gibbons, J.W. and R.D. Semlitsch. 1981. Terrestrial drift fences with pitfall traps: an effective technique for quantitative sampling of animal populations. Brimleyana 7:1-16.

Pechmann, J.H.K., R.D. Semlitsch, R.M. Lew and D.T. Mayack. 1984. Ecological Studies Related to the Construction of the Defense Waste Processing Facility on the Savannah River Plant; FY-1983-84 Annual Report. NTIS publ. SREL-17, UC-66e. 166 pp.

Pechmann, J.H.K., D.E. Scott and J.N. Knox. 1985. Ecological Studies Related to Congtruction of the Defense Waste Processing Facility on the Savannah River Plant. FY-1985 Annual Report. NTIS publ. SREL-19, UC-66e. 77 pp.

Pechmann, J.H.K., D.E. Scott, J.H. MCGregor, R.A. Estes and A.C. Chazal. 1993. Ecological Studies Related to Construction of the Defense Waste Processing Facility on the Savannah River Plant. FY-1989 and FY-1990 Annual Report. NTIS publ. SREL-66, UC-66e. 103 pp.

Pechmann, J.H.K., D.E. scott, R.D. Semlitsch, J.P. Caldwell, L. J. Vitt, and J. W. Gibbons. 1991. Declining amphibian populations: the problem of separating human impacts from natural fluctuations. Science 253:892-895.

Savannah River Ecology Laboratory. 1979. Ecological inventory for the Proposed AFR Site. Final Report, January 31, 1979. Report No. EY-76-C-09-0819.

Savannah River Ecology Laboratory. 1980. Annual Report, FY-1980. A biological 
inventory of the proposed site of the Defense Waste Processing Facility on the Savannah River Plant in Aiken, South Carolina. NTIS publ. SREL-7 UC-66e. $179 \mathrm{pp}$.

Scott, D.E., J.H.K. Pechmann, J.N. Knox, R.A. Esteg, and A.M. Dancewicz. 1986. Ecological studies Related to the Construction of the Defense Waste Processing Facility on the Savannah River Plant. FY-1986 Annual Report. Savannah River Ecology Laboratory. NTIS publ. SREL-32 UC-66e. 103 pp.

Sharitz, R.R. and J.W. Gibbons. 1982. The ecology of southeastern shrub bogs (Pocosins) and Carolina bays: a community profile. Fish and wildlife Service, U.S. Department of the Interior, Washington, D.C. FWS-OBS82/04. 93 pp.

United States Department of Energy. 1982. Final Environmental Impact Statement, Defense Waste Processing Facility, Savannah River Plant, Aiken, South Carolina. NTIS publ. DOE/EIS-0082 UC-70.

Vitt, L.J. 1981. Annual Report, FY-1981. A biological inventory of the proposed site of the Defense Waste Processing Facility on the Savannah River Plant in Aiken, South Carolina NTIS publ. SREL-8 UC-66e. 86 pp.

Vitt, L.J., R.D. Semlitsch and M.I. Cothran. 1982. Annual Report, FY-1982. A biological inventory of the proposed site of the Defense Waste Processing Facility on the Savannah River Plant in Aiken, South Carolina. NTIS publ. SREL-13 UC-66e. 146 pp. 


\section{WATER QUALITY MONITORING OF PERIPHERAI STREAMS}

Tracy K. Lynch and David E. Scott

\section{INTRODUCTION}

The Savannah River Ecology Laboratory initiated a water quality monitoring program in November 1982 to assess the potential impact of the DWPF construction activities on peripheral streams. Upper Three Runs (UTR) Creek, which receives $s-$ and $z$-area drainage, is the only major stream on the Savannah River site that has not been impacted significantly by thermal discharge.

In FY-1983 before construction began, baseline information was collected on the natural water quality characteristics of all streams that could be impacted plus additional control sites: UTR Creek, Crouch Branch, McQueen Branch, and Tinker Creek, a major upgtream tributary of UTR. Rough grading of the construction site began on 15 September 1983 (Pechmann et al. 1984). Data gathered after ground-breaking through september 1987 have been used to evaluate initial impacts during construction and the effectiveness of erosion control measures. Data collected from October 1987 to the present are used to assess stream recovery and the continued effectiveness of erosion control measures; major earthmoving and site preparation activities had been completed by this date.

\section{METHODS}

Site selection

The four streams mentioned above are part of the DWPF watershed (Fig. II-1). McQueen Branch is the principle drainage tributary from the construction area. Crouch Branch receives the outflow from DWPF sediment basin 1. These tributaries are the two primary streams leaving $s-$ and $z$-area, and are the streams most likely to be impacted by construction activity. Both Crouch Branch and McQueen Branch flow into UTR Creek and impacts on them could 
OWPF WATER QUALITY MONITORING SITES.
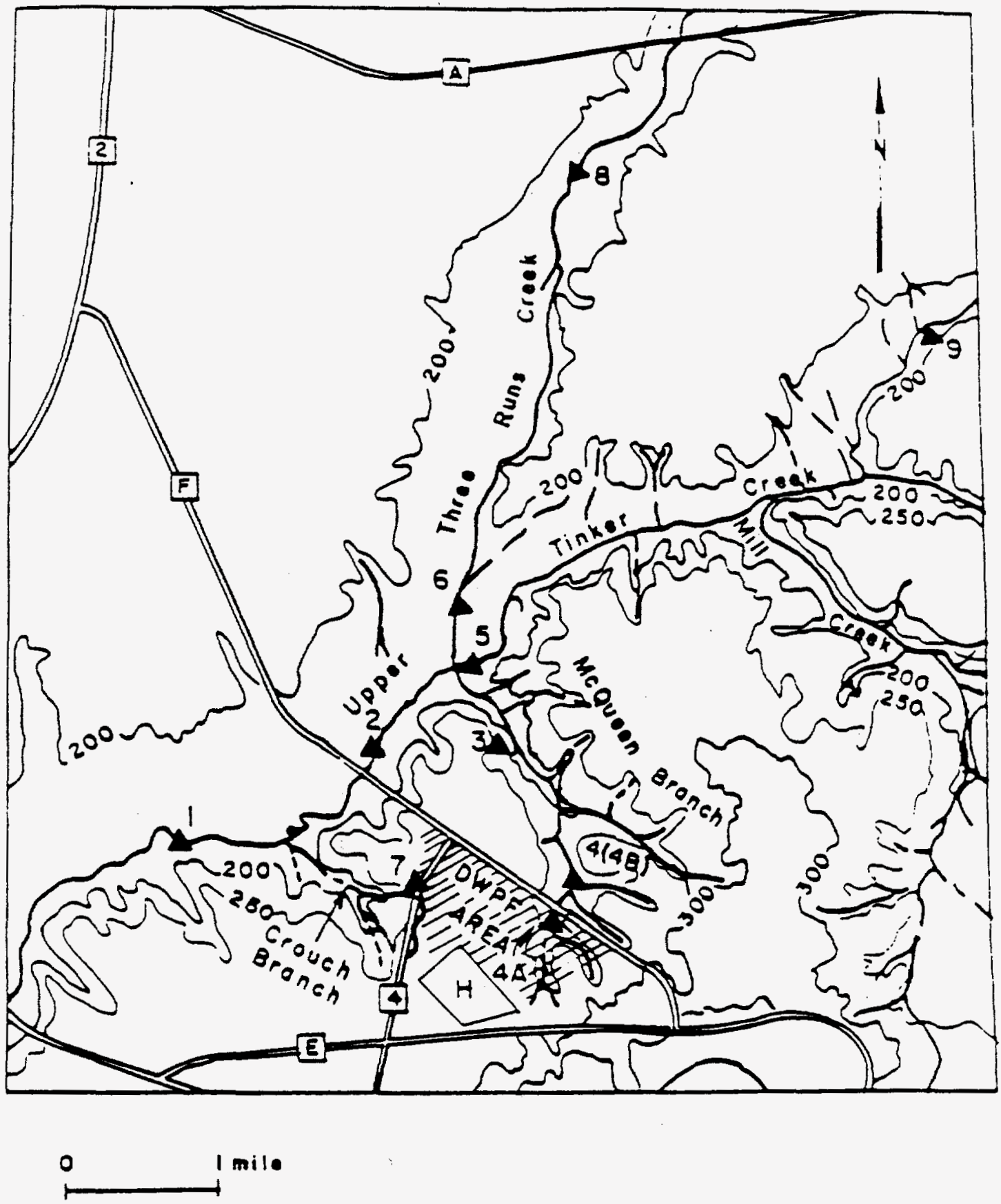

Figure II-1. DWPF Water Quality monitoring sites. 
potentially affect water quality in UTR creek. Tinker creek also flows into UTR just above the confluence of McQueen Branch and UTR. Similar in size to UTR Creek at their confluence, Tinker serves as an unimpacted control stream within the watershed. The SREL monitoring program previously focused on 10 sample sites in the UTR watershed; however, only nine sites are sampled currently (Fig. II-1). Two sites ( 3 and 4) are located on McQueen Branch. Site 3 is located approximately $2 \mathrm{~km}$ downstream of the construction area, and site 4 is located on the south side of road F. A third site on this Creek was sampled between November 1984 and September 1986 (Scott et al.1988). Site 7 is located on Crouch Branch at Road 4 approximately 122 m downstream from the outflow of sedimentation basin 1. Site 7 was sampled twice in FY-1982 and deactivated until FY-1986 after which it was sampled regularly. Sites 1 and 2 are located on UTR Creek below the confluence with Mcqueen Branch, and two more sites (sites 6 and 8 ) are located on UTR Creek above this confluence. Two gites (sites 5 and 9 ) are monitored on Tinker Creek upstream from the construction area. The latter four sites (sites 6, 8, 5, and 9) are unimpacted control sites.

\section{Sampling}

From the second to the seventh year of sampling (November 1983 September 1989), water quality monitoring was conducted monthly. During FY1983 (November 1982 - September 1983), sampling was conducted more frequently than in later years and with an emphasis on sampling during and after rainfall for the purpose of establishing existing water quality characteristics. Due to budgetary cuts, monitoring ceased between october 1989 and February 1990; however, monthly sampling resumed in March 1990. Thus, the first year (FY1983) includes data for 11 months and during FY- 1990 streams were sampled for seven months. Water quality measures from the remaining years are based on 12 months.

SREL personnel measured the following water quality variables: total suspended solids (TSS), percent ash, turbidity, and specific conductance. Total suspended solids is a measure of the dry weight of nonfilterable residue 
in each sample. Percent ash is a measure of the inorganic component of the TSS. The ratio of inorganic to organic matter may change relative to the specific stream inputs, e.g., eroded clays vs. leaf litter. Turbidity is based on measurements of refracted light and indicates the relative amount of undissolved particles in a stream sample. Specific conductance measures the ability of a sample to carry an electrical current and depends mostly on the level of dissolved salts (ions) present in the water. Stream profiles and flow measures were added to the routine sampling in october 1985 at three sites: 3 and 4 (Mcqueen Branch), and 9 (Tinker Creek).

Until February 1985, specific conductance was measured with a field conductivity bridge. Samples since February 1985 were analyzed in the laboratory using a sybron PM-10CB conductivity bridge or an Orion Research Conductivity Meter Model 101 (25 C). Turbidity was determined in the laboratory using a nephelometer which measured in Nephelometric Turbidity Units (NTU). Samples were analyzed for TSS and ash weight using EPA approved methods (US EPA, 1983). A rain gauge was placed adjacent to the DWPF construction site and monitored daily. Stream velocity ( $\mathrm{cm}$ per second) was measured using a Marsh-MCBirney portable flow meter. Stream velocity and flow were used to calculate TSS load.

In FY-1986 four plots were established on McQueen Branch to inventory the particle size composition and to observe composition changes over time. Based on the analysis of 2 years of such data a recommendation to terminate that portion of the DWPF monitoring project was made (Scott et al.1988) and adopted.

\section{Data Analysis}

For the statistical analysis of the water quality data, sampling sites were grouped into six watershed locations: the two McQueen Branch sites $(3$ and 4; McQueen), the two Tinker Creek sites (5 and 9 ; Tinker), the two UTR Creek sites above McQueen Branch ( 6 and 8 ; UTR-above), the UTR Creek site below Mcqueen Branch (2; UTR-middle), the Crouch Branch site (7; Crouch), and the UTR Creek site below Crouch Branch (1; UTR-below). General patterns observed 
in the data suggested further grouping of data based on rainfall one day prior to sampling. Data were grouped into three classes based on rainfall during the previous day: rainfall $\leq 0.1 \mathrm{~cm}$ (No Rain), rainfall $>0.1 \mathrm{~cm}$ but $\leq 1.0 \mathrm{~cm}$ (Low Rain), and rainfall $>1.0 \mathrm{~cm}$ (Bigh Rain, see scott et al. 1988). Data also were categorized based on the stages of the construction project: Before (1983), During (FY-1984 to FY-1987), and After (FY-1988 to FY-1993) Construction (see Introduction).

Analysis of variance (ANOVA) was used to assess the impact of DWPF construction on $s-$ and $z$-Area stream water quality. In order to increase homogeneity of variance and normality in the data, log transformations were performed on TSS, turbidity, specific conductivity, TSS load (kg per day per km2), and rainfall data. The transformations were made by adding 1.0 to each observation and then taking the natural $\log$ of the sum. The transformed variables and percent ash measurements were used in ANOVA models to test for effects of DWPF construction activities in each rainfall class (NO, Low, and High Rain).

Within the ANOVA models, a number of hypotheses were tested for each rainfall class. For each water quality variable (e.g., log TSS), statiatical tests determined whether or not there was a significant location effect (e.g., Did the locations differ in their level of TSS averaged across all construction periods?), a significant construction period effect (e.g., Did the construction periods differ in their level of TSS averaged across all locations?), and a significant location-by-construction interaction (e.g., Did TSS levels at some locations respond differently over the construction periods than at other locations?). This last test, the test of a location-by-construction interaction effect, is the primary test of whether construction activity has affected water quality in the DWPF peripheral streams.

This interaction test is depicted graphically in Figure II-2. In the example, a comparison of stream 1 vs. stream 2 reveals that stream 2 has higher levels of the measured variable, but both streams respond the same over 
the three construction periods, i.e., there is no location-by-construction interaction effect because the lines are parallel. However, when stream 1 is compared to stream 3, stream response is not the same during the construction periods; stream 3 increases more in the After Construction period than stream 1 , i.e., there is an interaction effect because the lines are not parallel. Uring the ANOVA models, specific comparisons were made between control locations (UTR-above and Tinker) and impacted locations (McQueen, Crouch, UTRmiddle, and UTR-below). These tests (statistical contrasts) were limited to particular comparisons of interest: UTR-above v8. UTR-middle, UTR-above vs. UTR-below, Tinker vs. McQueen, and Tinker vs. Crouch. Comparisons were made over three time intervals (Before vs. After, Before vs. During, and During vs. After Construction). Due to small sample sizes all Crouch Branch samples and all turbidity samples taken in the Before Construction period were omitted from the statigtical analyses. Data were analyzed using sas version 6.07 statistical package (SAS Institute Inc. 1985a, b). In the following results and discussion section graphical representations of the data accompany and illustrate statistical outcomes and trends. In addition, Appendix A lists numeric summaries of the water quality data by fiscal year and rainfall class, Appendix B listg $P$ values of ANOVA main effects, and Appendix C summarizes construction period means. 


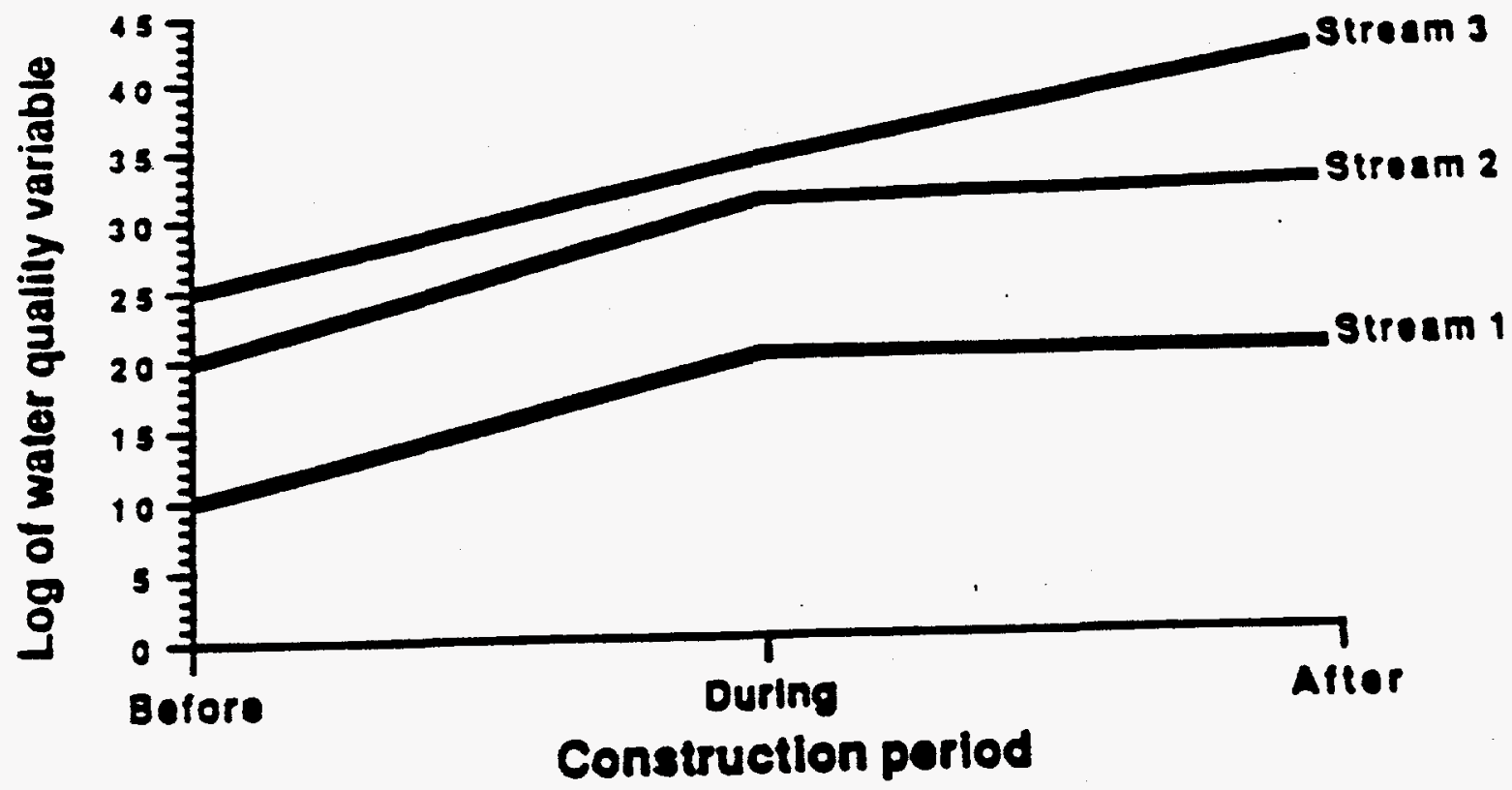

Figure II-2. Water quality variable (log-transformed) plotted for three construction periods (schematic representation of the interaction test in analysis of variance models). 


\section{RESULTS AND DISCUSBION}

\section{Introduction}

Log TSS values for each location were plotted against log rainfall levels one day prior to sampling for each construction period (Fig. II-3). These graphs illustrate several points. First, levels of Tss increase as rainfall increases, for all locations across all time periods. Second, TSs levels are generally higher During (DC) and After (AC) Construction than Before (BC) DWPF Construction, even in the No Rain class. These observations hold for streams that receive drainage directly from $s$ - and z-areas (Crouch Branch and McQueen Branch) as well as control locations (UTR-above and Tinker Creek). In addition, TSS levels in the smaller streams were higher than the larger streams irrespective of the construction period.

In both the No Rain and High Rain classes, the main effect of location was aignificant for TSS, opecific conductivity, percent ash, and turbidity. In the Low Rain class the location effect was also significant for all variables except TSS (Appendix B). In general, locations differed in their levels of all water quality variables averaged across construction periods.

The construction periods differed in their level of a given variable averaged over all locations. Construction period significantly affected levels of all water quality variables in the No Rain class. In general, in the No Rain class levels of the water quality variables in the s-Area peripheral streams were elevated in the During Construction Period, and have since declined. In the Low Rain class only specific conductance levels were significantly affected by construction period. In the High Rain class, only turbidity levels were significantly influenced by the construction period; effects on other variables were non-significant, perhaps due to the extreme variation in levels of all variables under high rainfall conditions. For the No Rain class, interpretation of the significant location and construction main effects is confounded by the significant location-by construction interaction.

Given these relationships, the location-by-construction period 


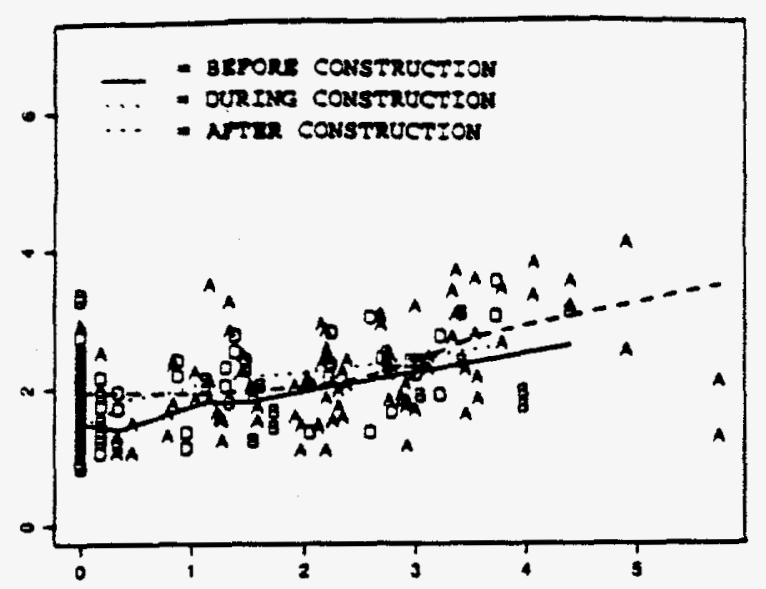

UTR MIDDLE

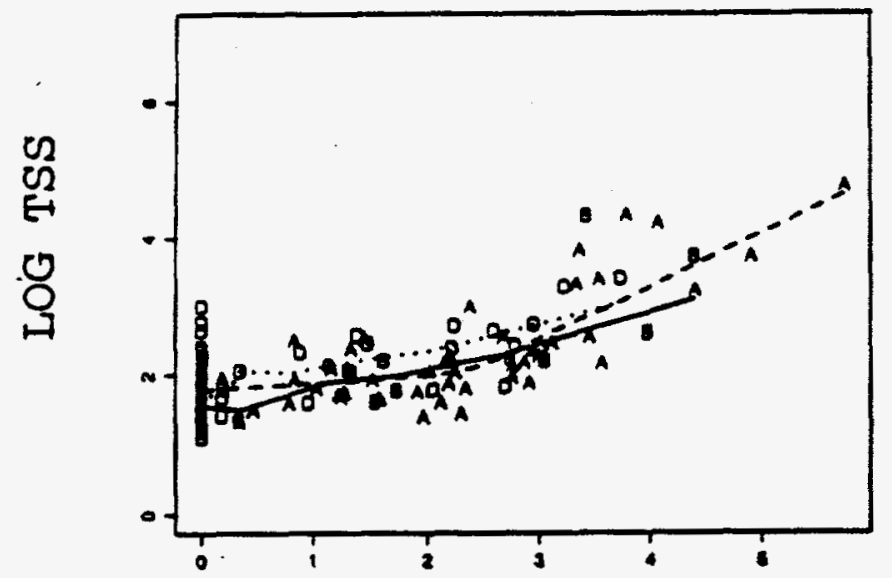

MCQUEEN BRANCB

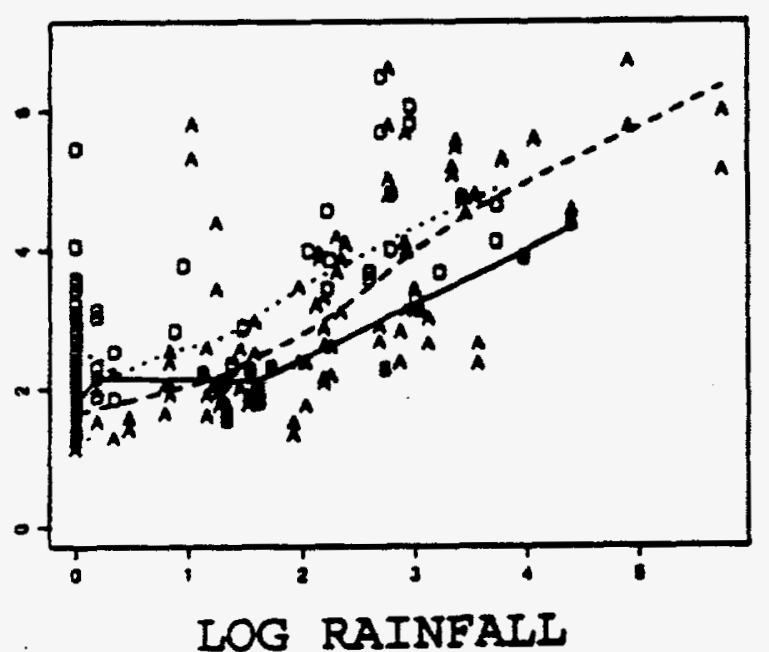

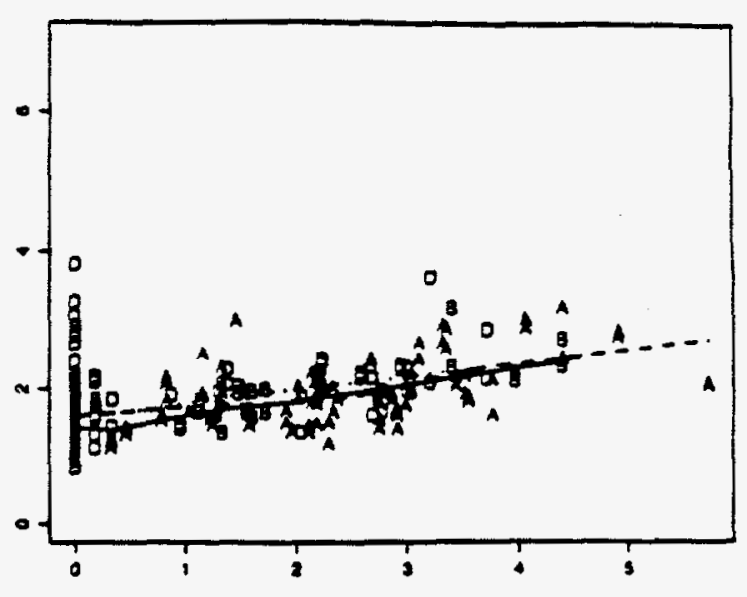

UTR BELON

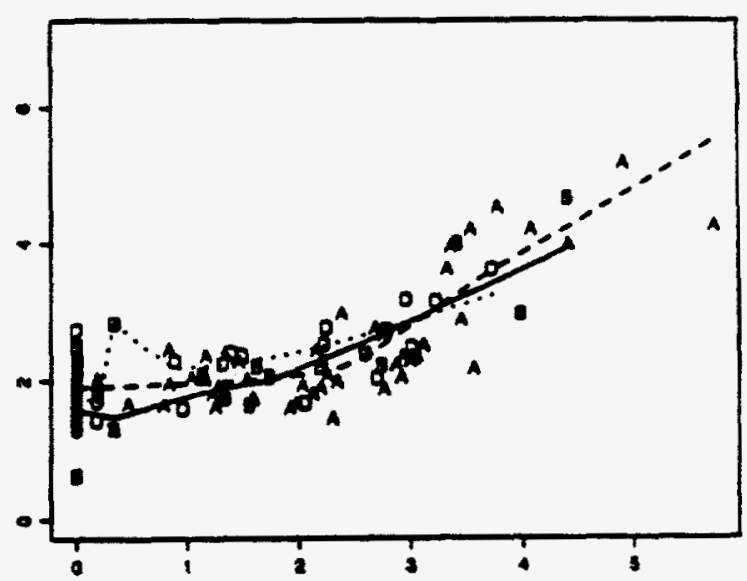

CROOCH BRANCH

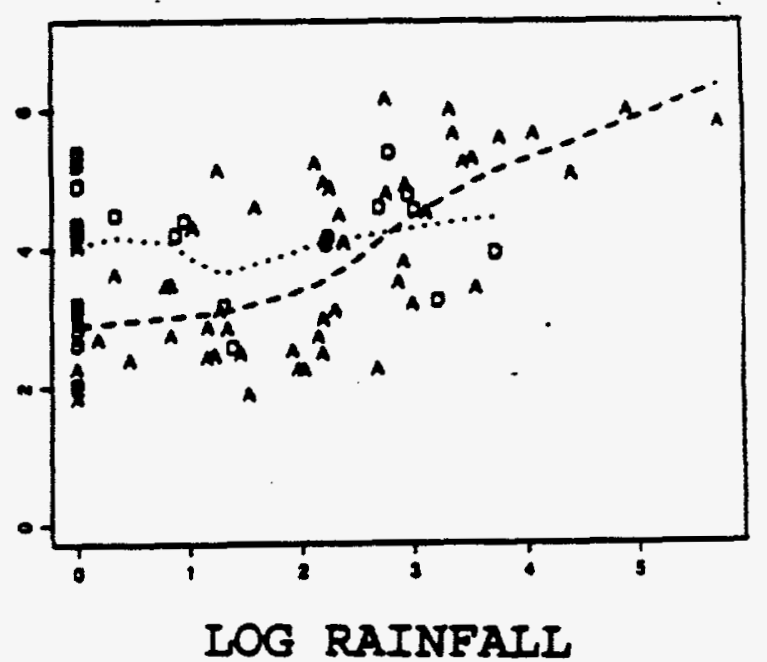

Pigure II-3. Log TSS levels plotted versus rainfall for each location. 
interaction term was used to test the effect of DWPF construction on stream water quality (as described in methods). Results of the ANOVA contrasts are listed and discussed by stream, beginning with the primary impact streams, McQueen Branch and Crouch Branch, and concluding with UTR Creek. Means for each construction period in each rainfall class are reported in Appendix $c$.

\section{McQueen Branch}

Water quality variables (i.e., TSs, turbidity, specific conductance, and percent ash) in McQueen Branch were compared to levels in the control stream, Tinker Creek, (Fig. II-4). TSS and turbidity levels rise more sharply as rainfall increases and percent ash levels are higher overall in McQueen Branch than in Tinker Creek. This difference is apparently a function of stream order; as a smaller steam, McQueen Branch is "flashier." The degree to which the overall water quality of McQueen Branch was influenced by DWPF construction activity was assessed using the specific statistical contrast tests.

TSS (Fig. II-5): No Rain - TSS levels rose moderately in Tinker Creek over the three construction periods. Levels in McQueen Branch were higher than the control BC, and increased significantly more DC $(p<0.005)$. In the After Construction period, TSS levels in McQueen Branch were reduced significantly compared to the decline observed in rinker creek $(p<0.0001)$, and returned to levels similar to McQueen Branch before construction (BC $x=5.38 ; A C x=$ 5.99).

Low Rain - TSS levels were higher in McQueen Branch than the control for all construction periods. TSS levels increased between BC and DC and declined slightly AC in both McQueen Branch and Tinker Creek. There were no significant differences in the response profiles of the two streams over time.

High Rain - TSS levels rose very slightly in Tinker Creek over the construction periods; levels increased in McQueen Branch DC, but declined slightly AC. Although the increase in TSS from BC to DC was more rapid in McQueen Branch than in Tinker Creek $(p<0.05)$, the decline from DC to AC 

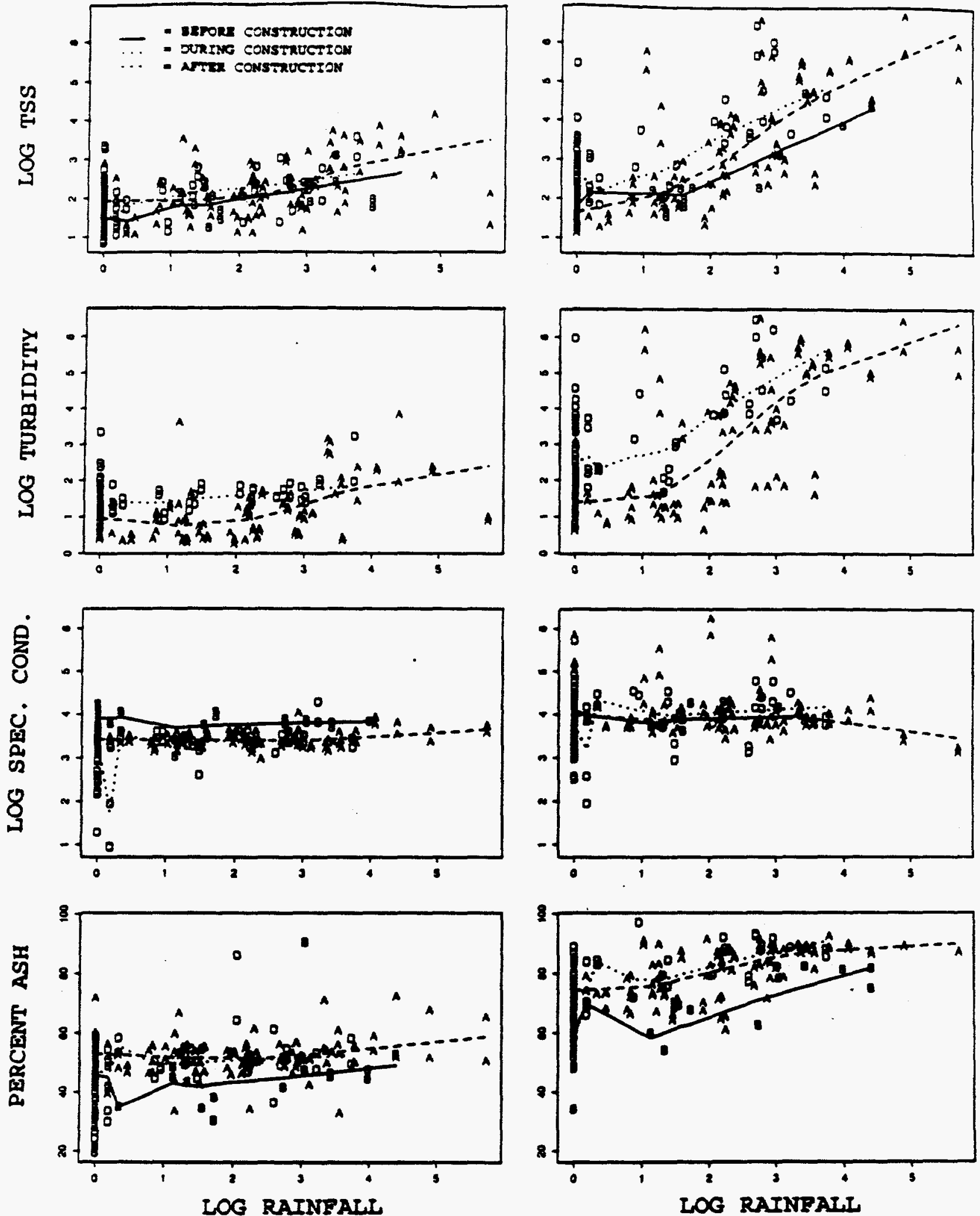

Figure II-4. Log TSs, log turbidity, log specific conductance, and percent ash plotted versus log rainfall for Tinker Creek and McQueen Branch. 

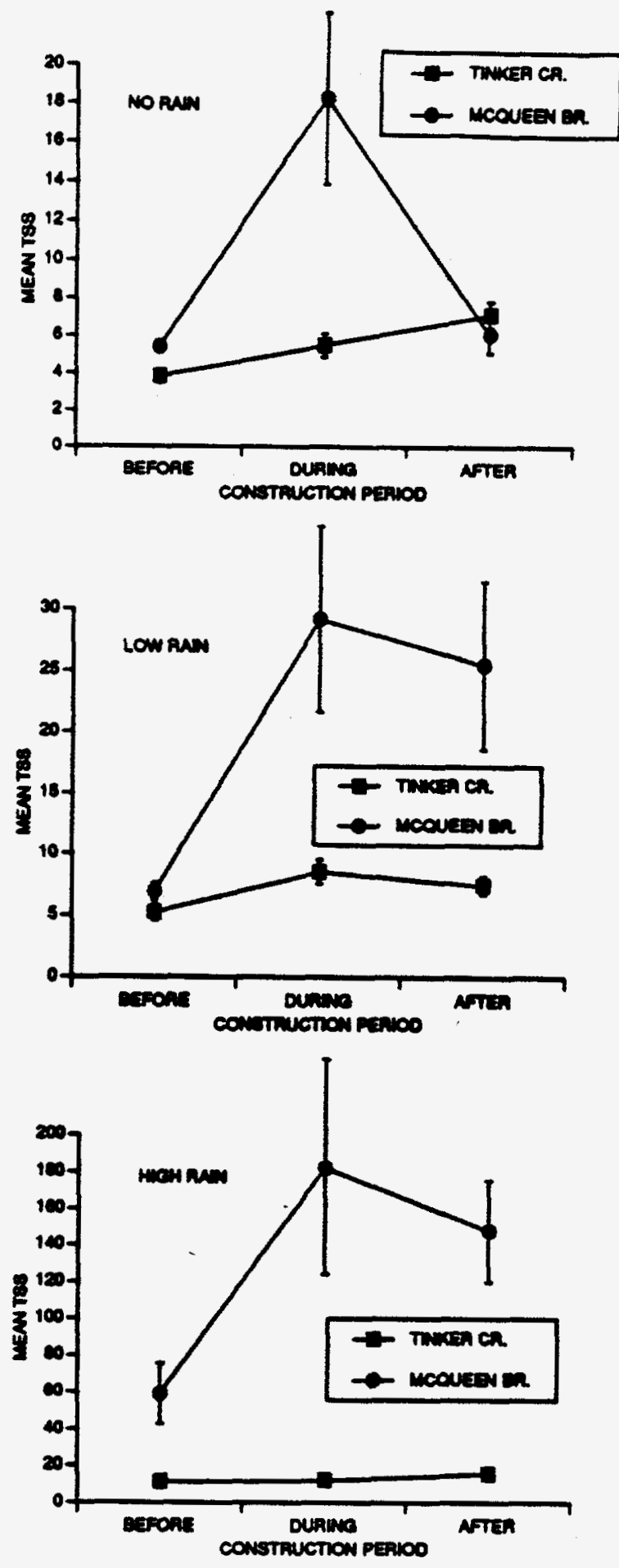

Figure II-5. Comparison of mean TSS levels in Tinker Creek (control) and Mcqueen Branch during the three construction periods. 
tended to be sigificantly greater $(p<0.08)$. Overall, there was no gignificant difference in the response profiles of McQueen Branch and Tinker Creek from BC to $A C$.

TURBIDITY (Fig. II-6): No Rain - Turbidity levels were higher BC and AC in McQueen Branch than Tinker Creek. Both creeks showed increases DC; however McQueen Branch decreased significantly more AC than Tinker Creek $(p<0.001)$.

Low and High Rain - Tinker Creek shows virtually no change in turbidity levels over time; McQueen Branch levels declined in the AC period compared to DC. The slight declines in turbidity in Mcqueen Branch from DC to AC were no different from the response profile of the control stream for either rainfall class $(p>0.20)$.

SPECIFIC COADUCTANCE (Fig. II-7): No Rain - McQueen Branch specific conductance was higher than Tinker Creek during all construction periods. Specific conductance levels in Tinker Creek fell from BC to DC, but the response proflle in Mcqueen Branch during the same time interval showed significantly less of a decline $(p<0.01)$. Specific conductance levels in both creeks increased at the same rate from DC to AC. Comparing responses from $B C$ to AC, McQueen Branch and Tinker Creek responded differently ( $P<0.01$ ), with levels in McQueen increasing as levels in Tinker decreased.

Low Rain - Specific conductance was higher during all construction periods in Mcqueen Branch than in Tinker Creek. Levels decreased DC and rose slightly AC in Tinker, while levels increased moderately throughout all time periods in McQueen Branch. The specific conductance response profile in McQueen Branch differed significantly from Tinker Creek from BC to AC (p < $0.03)$.

High Rain - McQueen's Branch specific conductance was higher than Tinker Creek for all construction periods. Specific conductance levels in Tinker Creek declined throught both time intervals. McQueen Branch levels rose slightly DC and fell slightly AC. There was no significant difference in the 

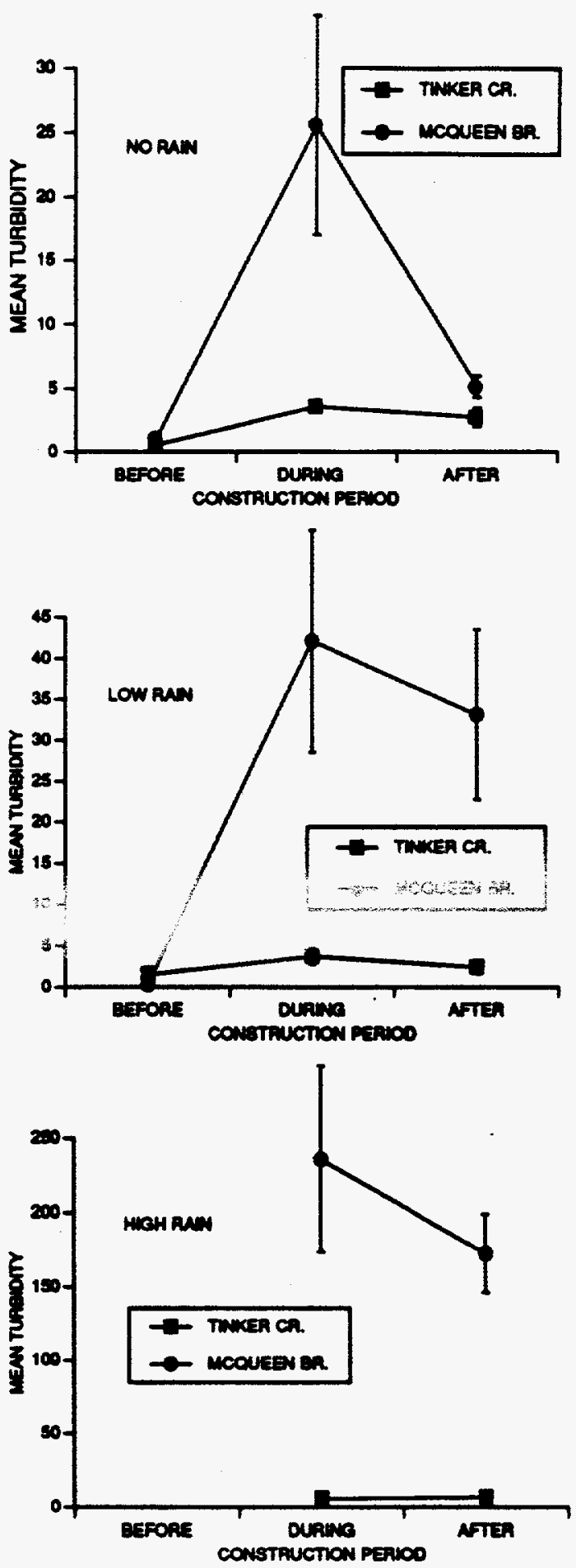

Figure II-6, Comparison of the nean turbidity levels in Iinker creek (control) and McQueen Branch during the three construction periods. 

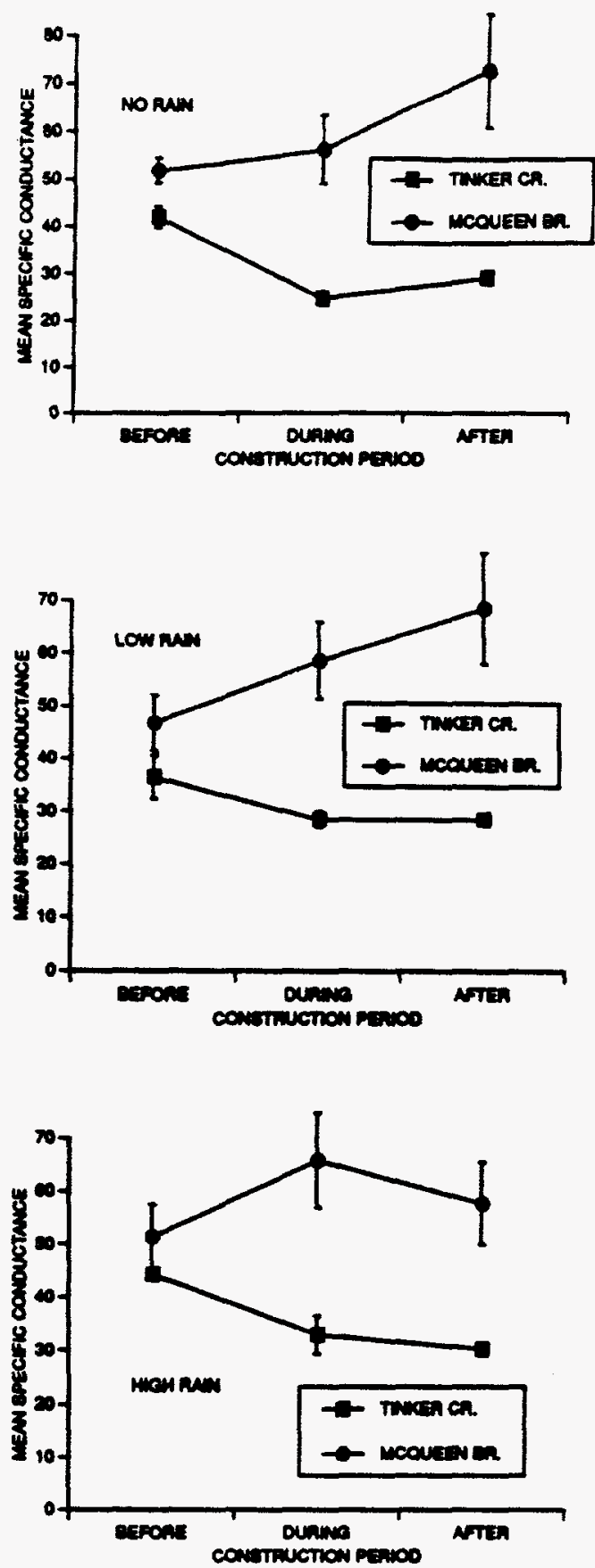

Figure II-7. Comparison of mean specific conductance levels in Tinker Creek (control) and Mcqueen Branch during three construction periods. 

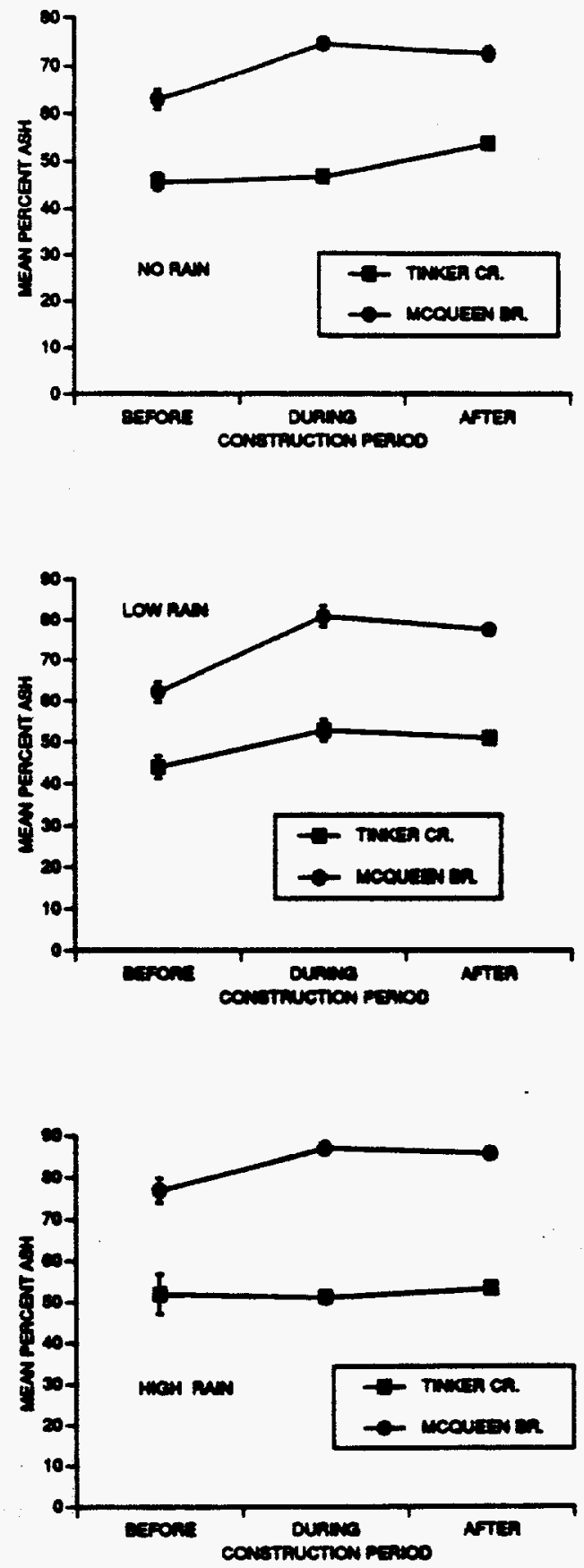

Figure II-8. Comparison of mean percent ash levels in Tinker Creek (control) and Kcqueen Branch during three construction periods. 
overall responses between the two streams.

PERCENT ASH (Fig. II-8): No Rain - Percent ash levels increased moderately in Tinker Creek over the three construction periods. Levels in Mcqueen Branch began higher than Tinker Creek and rose significantly more in the DC period ( $p$ $<0.001)$. Response profiles from DC to AC were also different $(p<0.001)$; percent ash continued to rise in Tinker Creek, but showed a slight decline in McQueen Branch.

Low Rain - Percent ash levels increased DC and decreased slightly AC in both Mcqueen Branch and Tinker Creek. Levels in Mcqueen Branch were higher than Tinker Creek over all time periods. There were no significant differences in responses between McQueen Branch and Tinker Creek over construction periods.

High Rain - Levels of percent ash in Tinker Creek decreased DC and increased slightly AC. Levels in McQueen Branch were higher than Tinker Creek BC and increased significantly DC $(p<0.02)$. Both streams had increased percent ash AC when compared to BC levels; the overall increase in McQueen Branch was significantly greater than the increase at Tinker Creek $(p<0.05)$. The results listed above provide evidence that Mcqueen Branch clearly was affected by DWPF construction-site runoff between the BC and DC period. These effects are evident in the TSS, specific conductivity and percent ash variables. However, there is also evidence that impacts have been ameliorated in the years since construction was completed. Turbidity and TSS levels under No Rain conditions were similar AC to BC levels (Figure II-5 and II-6). Under Low and High Rain conditions, however, TSS levels appear to remain elevated. Levels of other water quality variables also remained elevated AC in McQueen Branch in most cases; generally there was a slight decrease from DC levels. Given 10 possible comparisons of overall response profiles between BC and AC periods, four showed a significant location-by-construction interaction, indicating that McQueen Branch responded differently between BC and AC periods than Tinker Creek. Six comparisons were significant between the BC and DC periods. Three (of 12 possible, including turbidity) comparisons were 
significant between the DC and $A C$ periods and all of these occurred under No Rain conditions. The differences may be attributed to increases in the control stream versus little to no increase (and a decrease in one instance) in McQueen Branch. Some recovery of the tributary seems evident in the AC period.

\section{Crouch Branch}

Crouch Branch is the second primary impact site below the DWPF construction area. Like McQueen Branch, it is a small tributary that readily fills under High Rain conditions. Few data are available for this site BC; however, DC and AC data are used to test the location-by-construction period interaction between Crouch Branch and Tinker Creek for each water quality variable (Fig. II-9).

TSS (Fig. II-10): Wo Rain - Tinker Creek showed a slight increase in TSS from DC to AC. Crouch Branch TSS levels were considerably higher DC and AC although Crouch Branch showed a decrease AC. The difference in TSS level response between DC and AC for the two streams was gignificant $(p<0.0001)$. AC levels remained higher in Crouch Branch than in Tinker Creek.

Low and High Rain - The TSS response profiles for Crouch Branch and Tinker Creek were similar for both rain classes. In the Low Rain class, TSS levels in Crouch Branch decreased slightly AC compared to DC, as did levels in Tinker Creek. In the High Rain class, TSS levels in both creeks rose from the DC to the AC period. There was no significant location-by-construction period interaction for either rain class.

TURBIDITY (Fig. II-11): No Rain - Both Tinker Creek and Crouch Branch turbidity levels decreased between the DC and AC periods with Crouch Branch decreasing significantly more sharply ( $p<0.0001$ ) than Tinker Creek. Turbidity levels in Crouch Branch were higher than in the control stream during both time periods.

Low Rain - Turbidity levels in Crouch Branch and Tinker Creek decreased AC from the DC period; the response profiles of the two streams were not significantly different. 
High Rain - Tinker Creek turbidity levels remained relatively constant, while Crouch Branch levels increased from the DC to AC period. These responses were not significantly different, however.

SPECIFIC CONDUCTANCE (Fig. II-12): No, Low, and High Rain - Tinker Creek specific conductance levels remained relatively constant from the DC to the AC period under all rainfall conditions. For the No and Low Rain classes, specific conductance levels in Crouch Branch also were constant. Under High Rain conditions levels in Crouch Branch decreased slightly; response profiles of the two creeks were not significantly different.

PERCENT ASH (Fig. II-13): No Rain - Percent ash levels increased in Tinker Creek from the DC to the AC period. Levels in Crouch Branch decreased significantly during that same period compared to Tinker creek ( $p<0.001)$.

Low Rain - Both Tinker Creek and Crouch Branch showed slight decreases in percent ash levels from DC to the AC period; reaponse profiles were no different.

High Rain - Crouch Branch percent ash levels were considerably higher DC and AC than Tinker Creek. Levels in both creeks increased slightly AC from DC levels. Changes were non-significant.

Because few data are available for Crouch Branch in the Before Construction period, and because Crouch Branch is the smallest stream in the DWPF watershed, conclusions about overall changes in the stream are difficult to draw. However, the data do provide considerable evidence that Crouch Branch has been affected adversely by DWPF construction. First, for the High Rain class, levels of TSS, turbidity and percent ash increased in Crouch Branch during the AC period. Secondly, DC and AC levels in Crouch Branch are higher than every other sampled location in the watershed for each parameter measured in every rain class. Thirdly, significant decreases in TSS, turbidity, and percent ash levels are evident in the No Rain class during the AC period. Without the erosion input that occurs with low and high rainfall, Crouch Branch appears to show better water quality levels. Because it lies below a sedimentation basin, Crouch Branch water quality is a good measure of the 

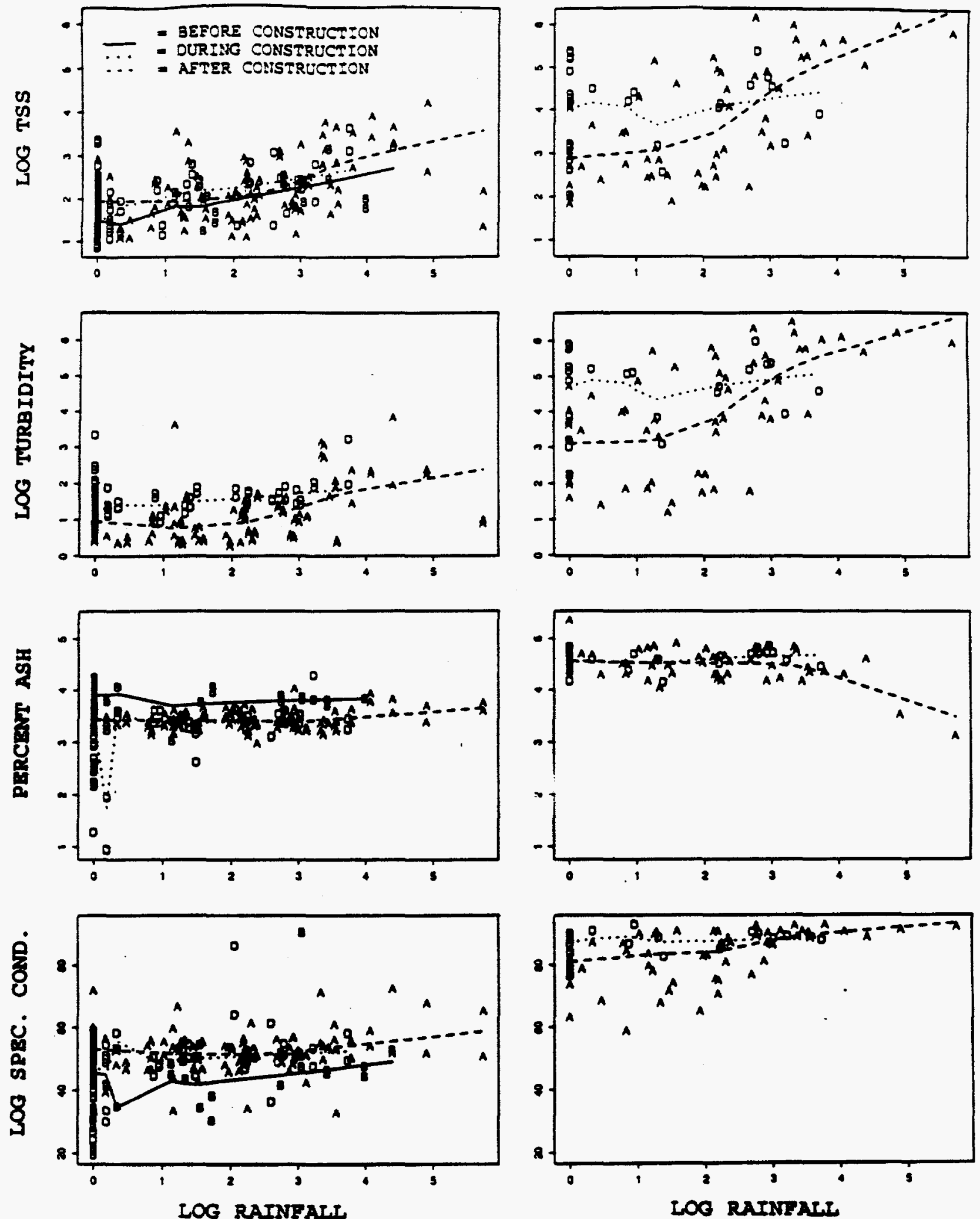

Figure II-9. Iog ISs, $\log$ turbidity, $\log$ specific conductance, and percent ash levels plotted versus rainfall for Tinker Creek and Crouch Branch. 

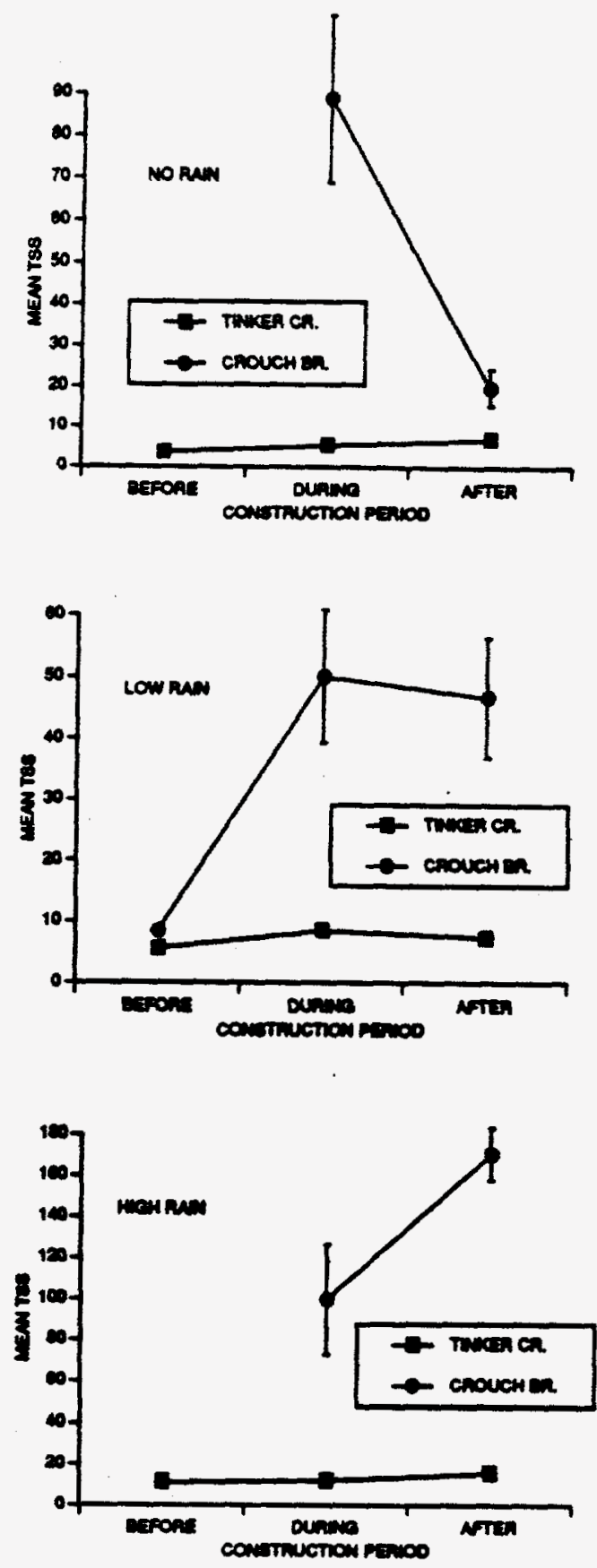

Figure II-10. Comparison of mean Tss levels in Tinker Creak (control) and Crouch Branch during three construction periods. 

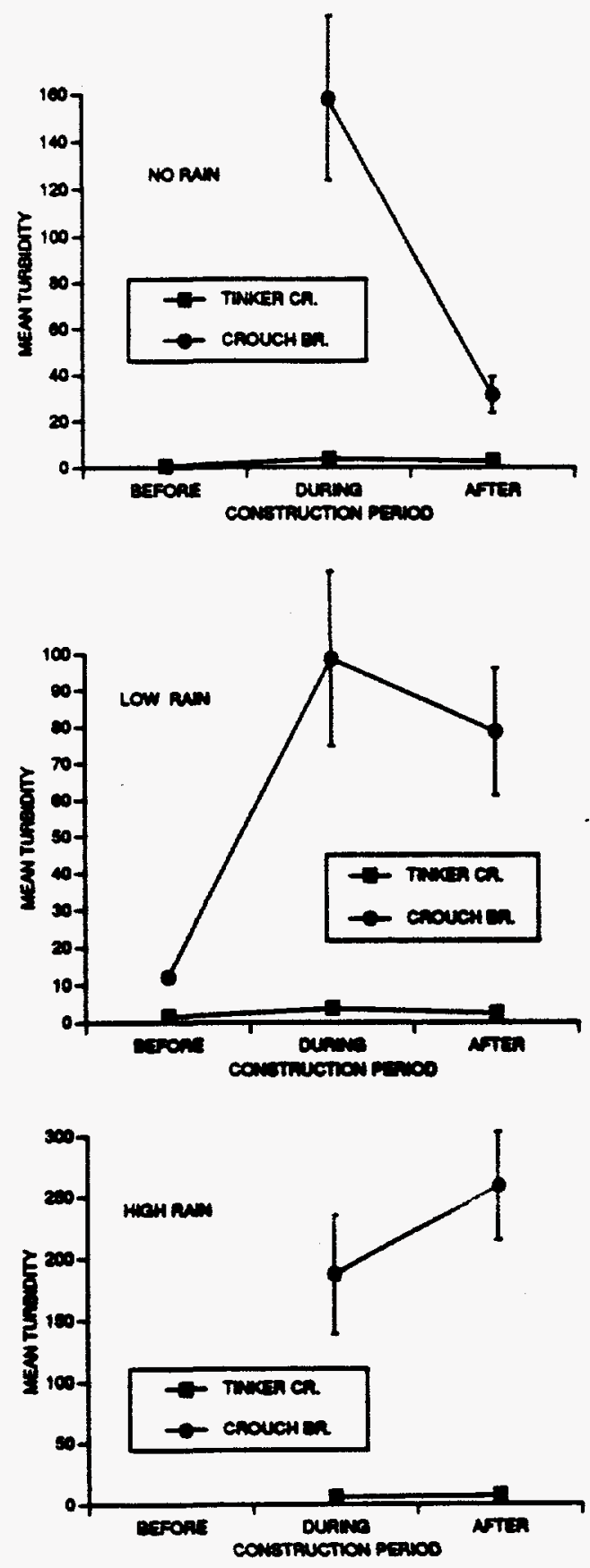

Figure II-11. Comparison of mean turbidity levels in Tinker Creek (control) and Crouch Branch during three construction periods. 

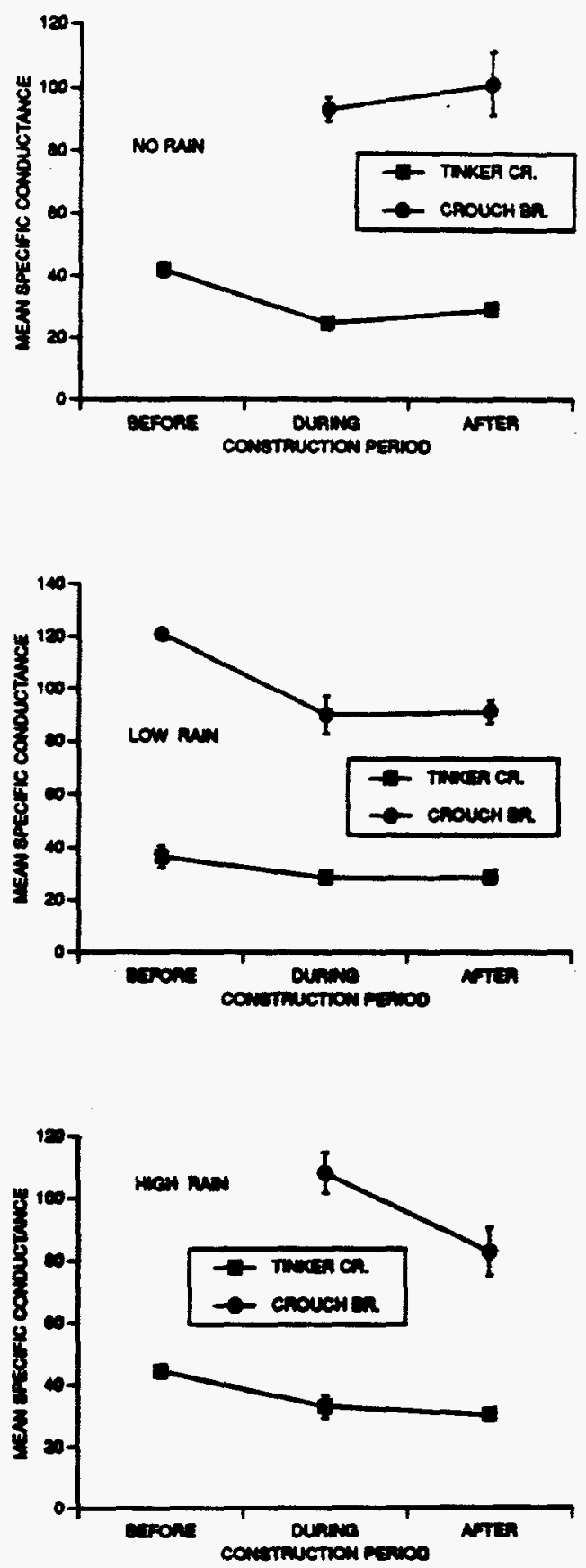

Figure II-12. Comparison of mean specific conductance levels in Tinker Creek (control) and Crouch Branch during three construction periods. 

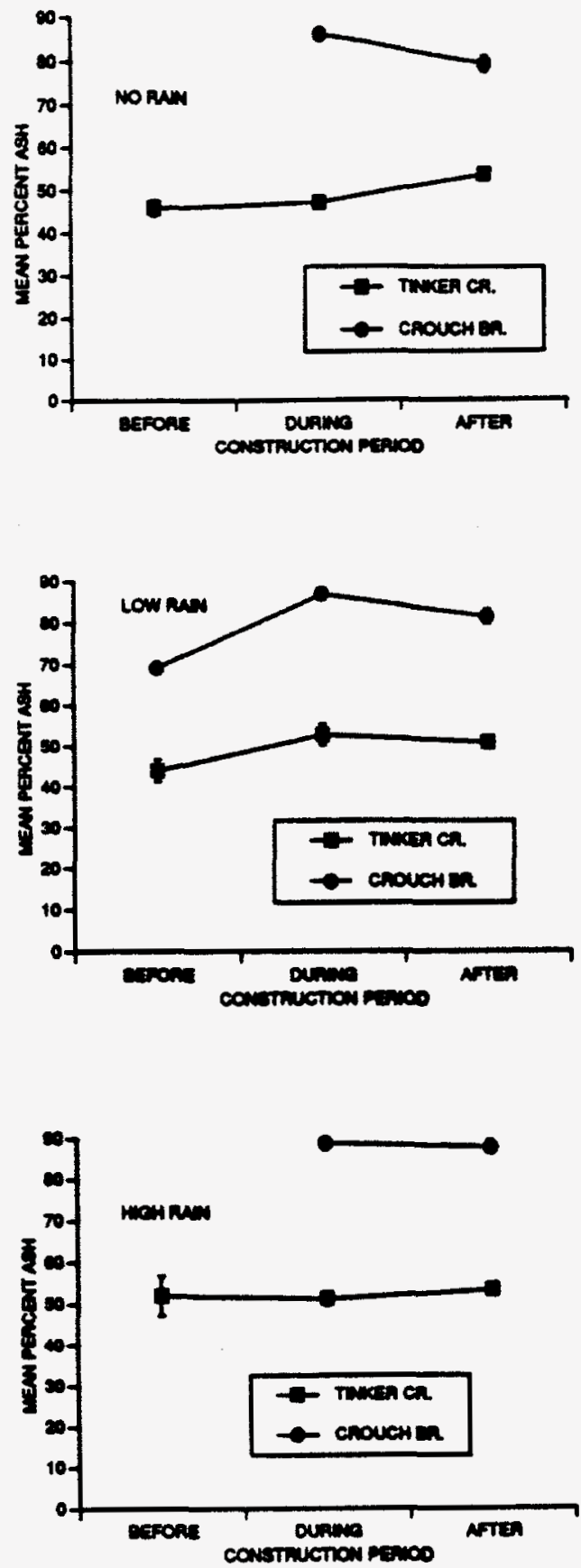

Figure II-13. Comparison of mean percent ash levels in Tinker Creek (control) and Crouch Branch during three construction periods. 
effectiveness of the basin's performance. As the basin fills with sediment it becomes less able to hold construction area runoff under rainy conditions, and consequently higher silt levels will occur in the stream below. The elevated levels of TSS and turbidity in Crouch Branch may reflect this condition. 
Upper Three Runs Creek

A principle concern of this monitoring program is to assess the potential impacts of DWPF construction on water quality in UTR Creek. Two contrast pairs were made: UTR-above vs. UTR-middle (effects below the McQueen Branch and Tinker creek confluences) and UTR-above vs. UTR-below (effects below the confluence of Crouch Branch; Figs. II-14 and II-15). TSS (Fig. II-16): No and Low Rain - Trends in the three UTR Creek locations were similar. TSS levels rose DC and fell AC except for after-construction Tss levels in UTR-below which increased slightly in No Rain conditions. Some trends hinted at potential erosional input to UTR; UTR-middle and UTR-below TSS levels were higher than UTR-above levels during all periods, levels were most different in the $A C$ period under baseflow (No Rain) conditions, and the largest difference occurred between UTR-below (which received inputs from McQueen Branch, Crouch Branch, and Tinker Creek) and the control location (UTR-above) during the AC period. In addition, all AC levels were higher than BC levels, but elevated levels also occurred in the control section of the stream (UTR-above). No response profiles were significantly different between the three UTR locations.

High Rain - UTR-above showed little change in TsS level over the three construction periods and had the lowest Tss levels of all three sites during all time periods. UTR-middle and -below showed a decrease DC in TSS levels, but both increased during the AC period to levels comparable to BC at each location. Changes were non-significant.

TURBIDITY (Pig. II-17) No and Iow Rain - All locations on UTR Creek showed a non-significant decrease in turbidity AC from DC levels.

Bigh Rain - Turbidity levels increased in UTR-above and -below from the DC to AC period, while levels in UTR-middle decreased. These responses were also non significant.

SPECIFIC CONDUCTAUCE (Fig. II-18): No and Low Rain - For all locations on UTR Creek, specific conductance decreased from the BC to DC period and increased slightly AC. Levels AC were below levels BC. No changes were statistically significant. 

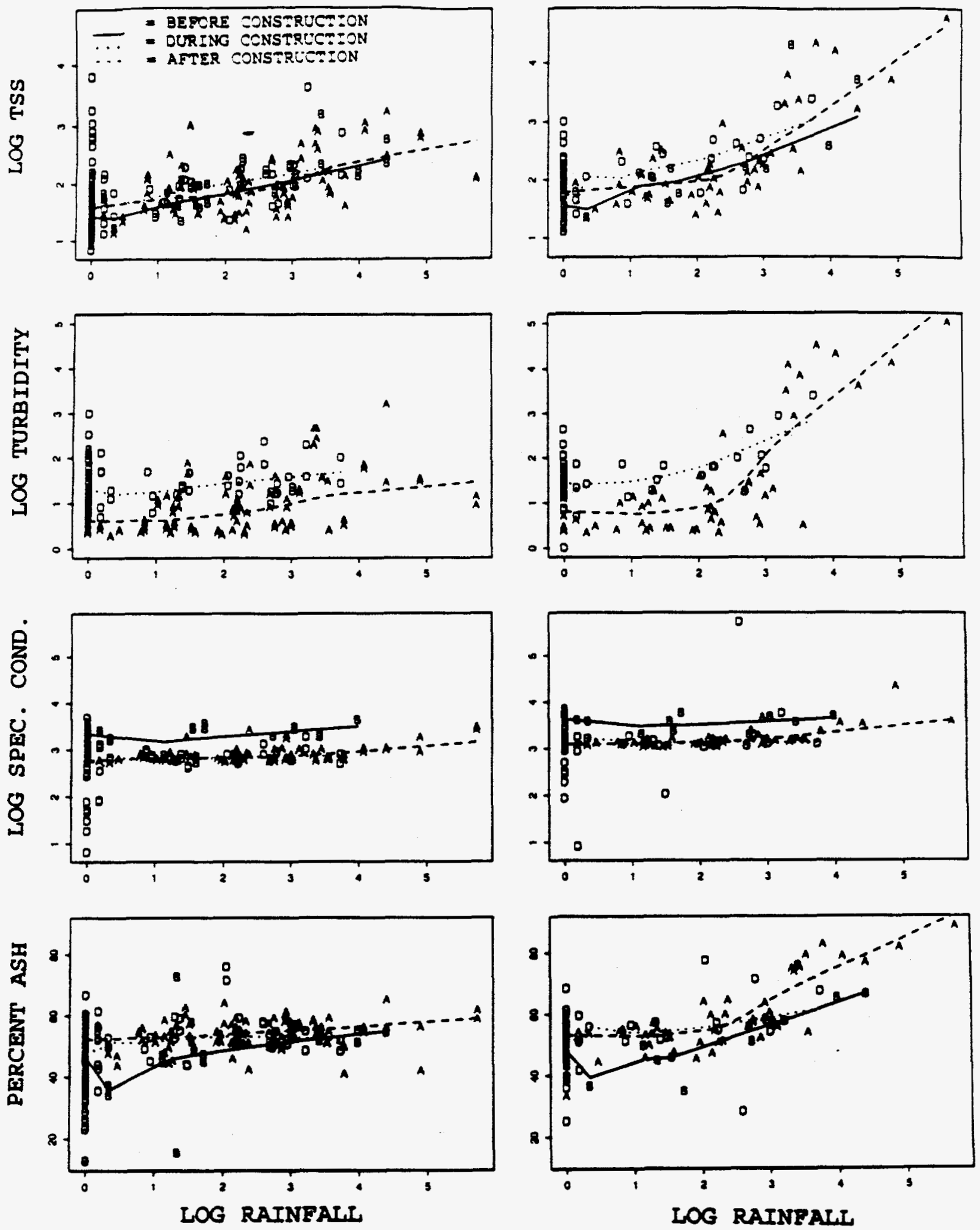

Figure II-14. Log Tss, $\log$ turbidity, $\log$ specific conductance, and percent ash levels plotted versus rainfall for UTR-above and UTR-aiddle. 

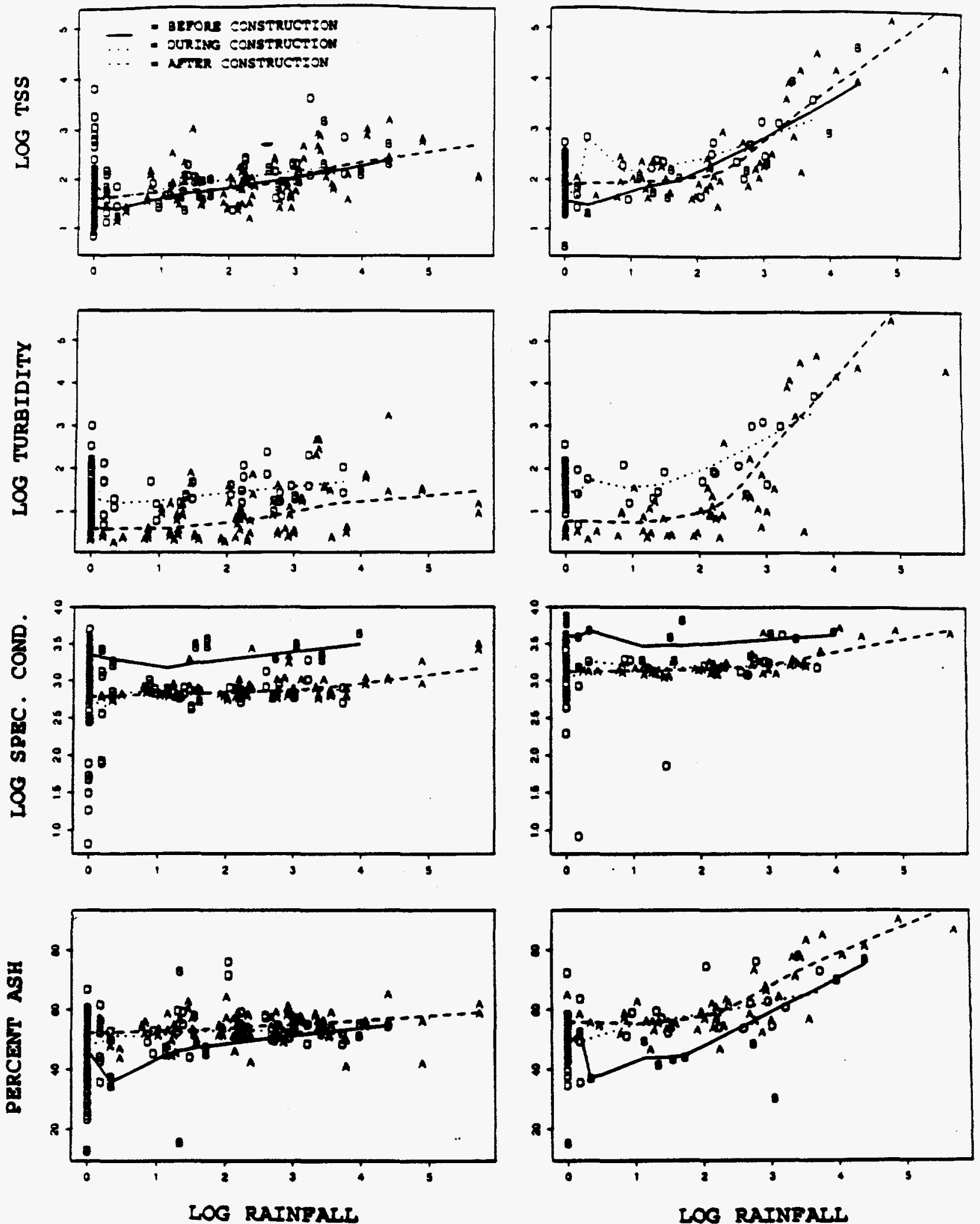

Figure II-15. Log TSS, log turbidity, log specific conductance, and percent ash levels plotted versus rainfall for UIR-above and UIR-balow. 

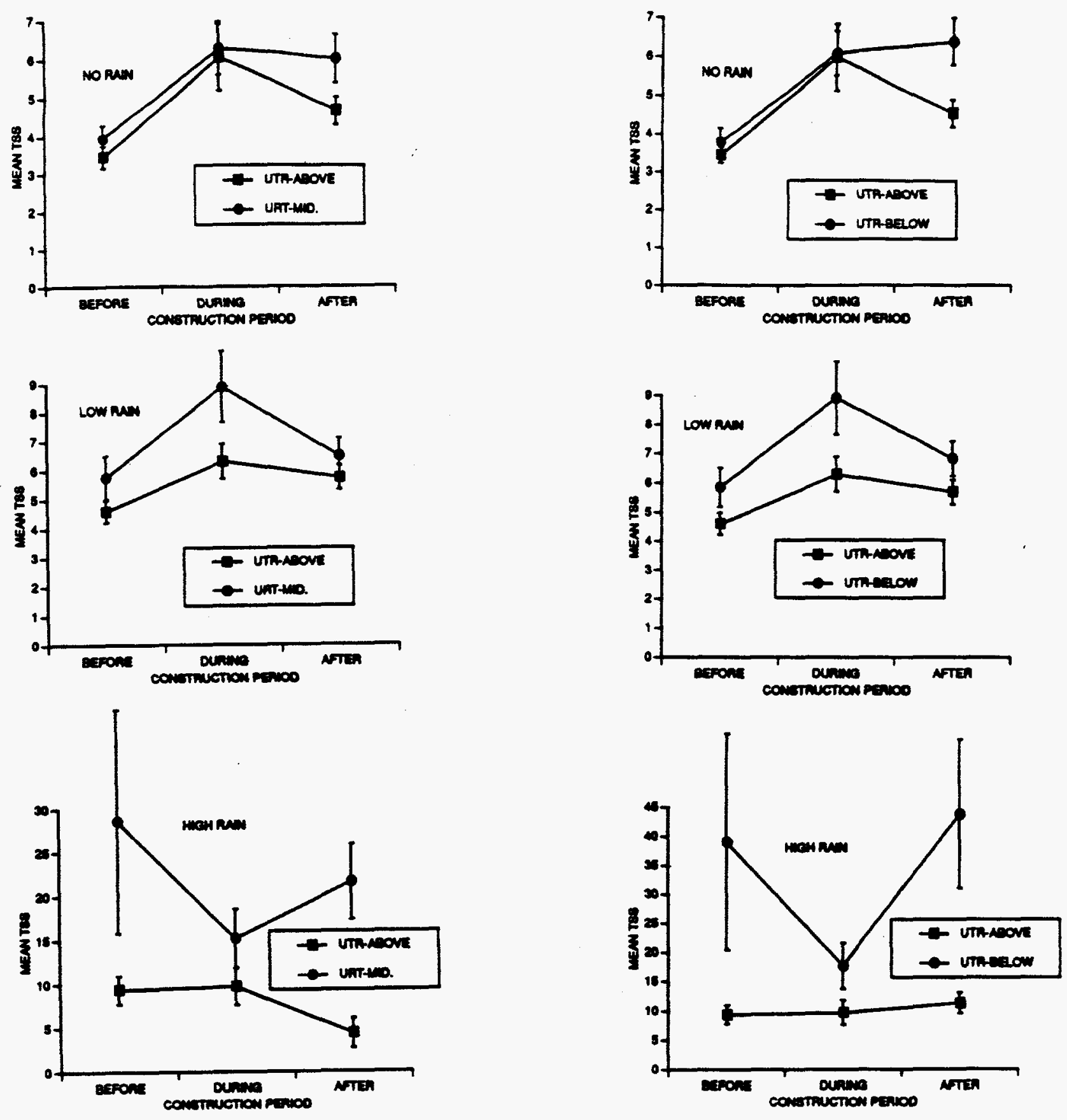

Figure II-16. Comparison of mean TSS levels in Upper Three Runs Creek-above (control) and Upper Three Runs Creak-niddle and -below during three construction periods. 

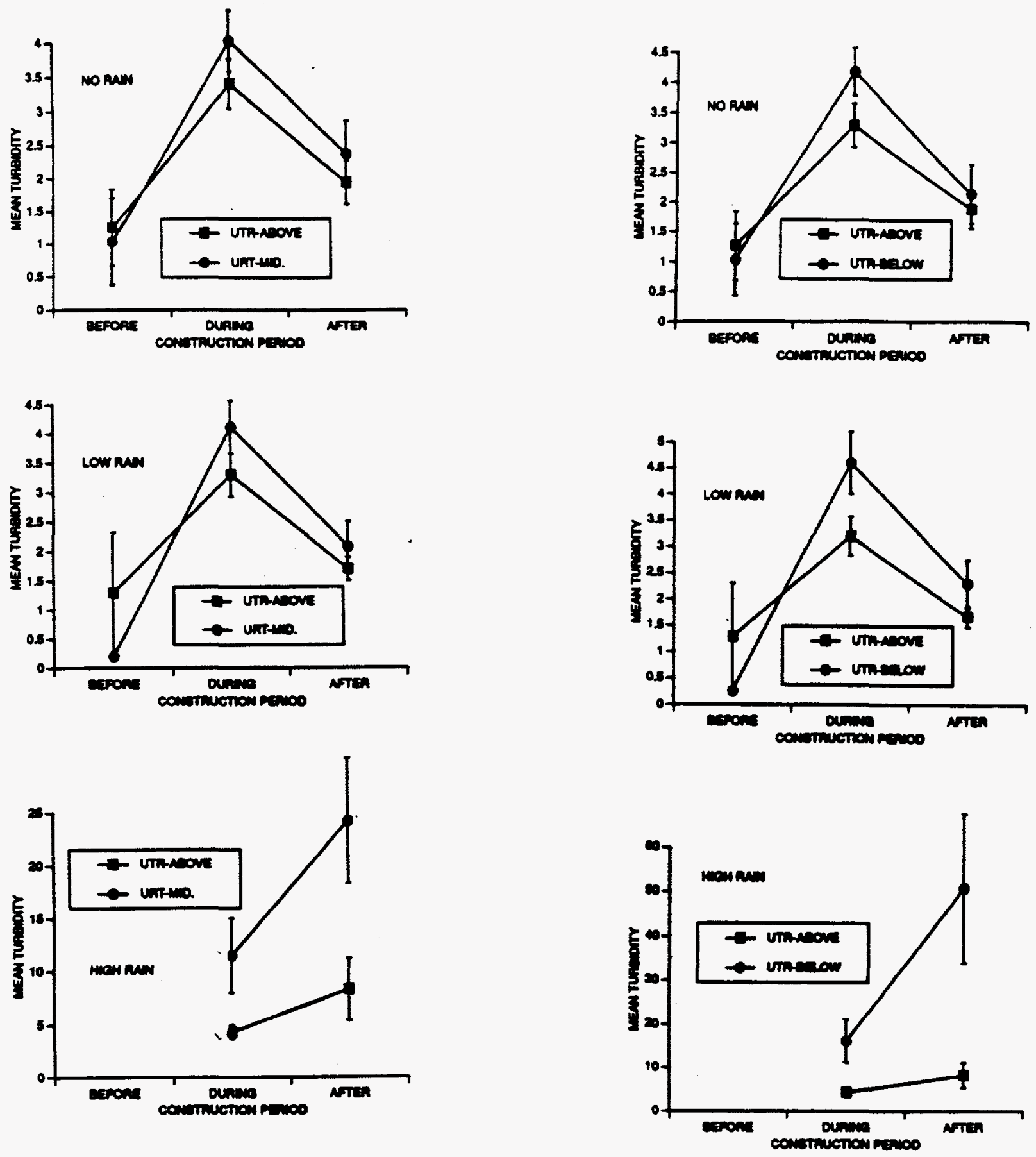

Figure II-17. Comparison of mean turbidity levels in Upper Three Runs Creekabove (control) and Upper Three Runs Creek-middle and -below during three construction periods. 

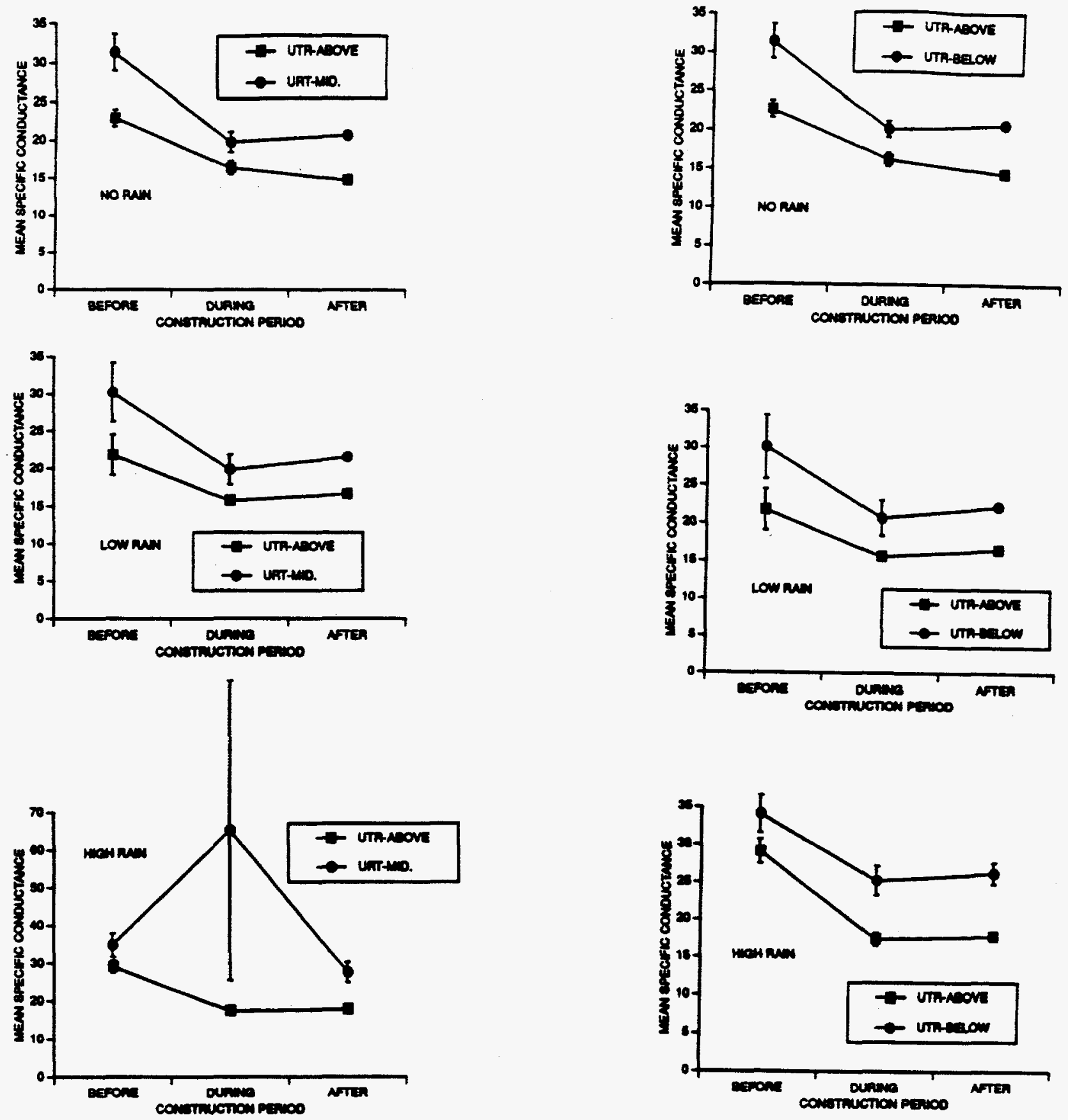

Figure II-18. Comparison of mean specific conductance levels in Upper Three Runs Creek-above (control) and Upper Three Runs Creek-niddle and -below during three construction periods. 
High Rain - UTR-above and -below decreased from BC to DC and increased AC. UTR-middle showed an increase DC followed by a decrease AC to BC levels. The only significant interaction involved the UTR-above and middle sites during the $\mathrm{BC}$ and $\mathrm{DC}$ periods.

PERCENTI ASE

UIR-Above Ys. UTR-Kiddle (Fig. II-19): No and Low Rain - Both locations (UTR-above and UTR middle) showed increasing percent ash levels over the three periods except levels at UTR-middle decreased slightly between DC and AC. Percent ash at UTR-middle was higher BC and levels at UTR-above were higher AC. No changes were significant.

High Rain - Percent ash levels at both UTR-above and -middle decreased DC and rose during the AC period to levels higher than the BC levels. The changes were non-significant.

UMR-gbove Yg. UTR-Below: No Rain - Percent ash levels in UTR-above and -below followed parallel patterns of increase during the three periods. Percent ash levels for UTR-above remained below that of UTR-below.

Low Rain - Percent ash levels in UTR-below increased DC and decreased AC while UTR-above increased over all construction periods. Both locations ended with percent ash levels above BC levels.

High Rain - Percent ash levels in UTR-above decreased DC and rose only slightly AC. Levels in UTR-below increased significantly DC (p < 0.03 ) and increased further AC. When compared to UTR-above, both of these changes were significantly different (BC to DC, $p<0.003$; DC to AC, $p<0.004$ ).

The data collected over the eleven years of this study show an increase in TSS, turbidity, and percent ash levels in UTR Creek; however, this increase cannot be attributed to inputs from McQueen Branch and Crouch Branch alone. Comparisons of UTR Creek above the construction site to two locations below did not provide evidence that the $s-$ and $z$-area construction raised Tss or other parameter levels in UTR Creek. One possible explanation for the observed decrease in water quality in UTR Creek is the effects of additive inputs. Possible contributors to increased TSS levels begides DWPF construction might 

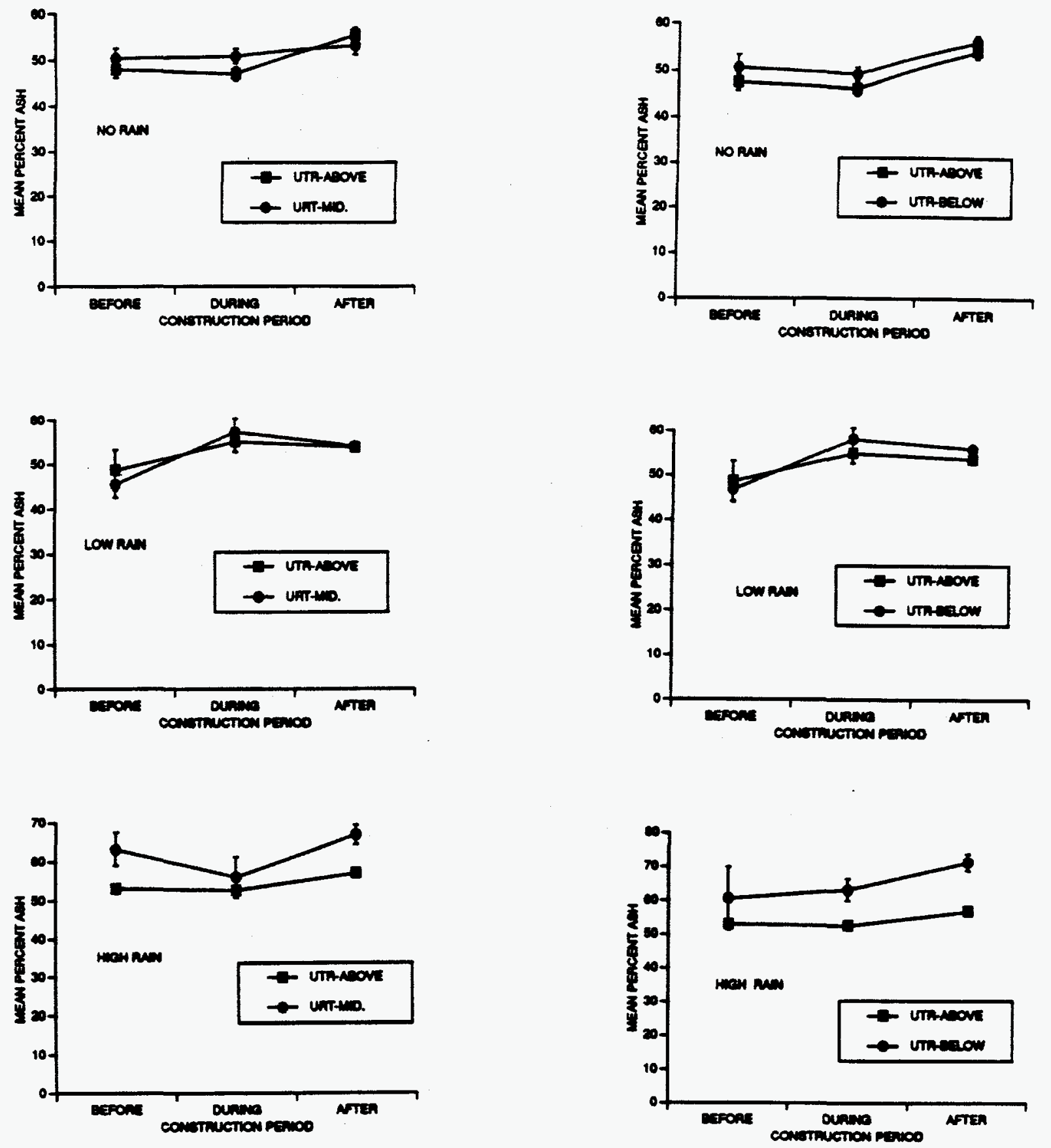

Figure II-19. Comparison of mean percent ash levels in Upper Three Runs Creek-above (control) and Upper Three Runs Creek-middle and -below during three construction periods. 
be: increases in off-plant construction activities upstream on UTR creek, increases due to clogged road drainage, flash flooding, or other unnoted disturbances along the creek drainage. A combination of such activities, in conjunction with the s- and z-area input, may have contributed to this alteration in water quality over time. For non-point pollutants, such as erosion, multiple effects are difficult to identify and accurately assess; however, based on this study, water quality in UTR Creek has not been significantly affected by the construction site input from Mcqueen Branch or Crouch Branch.

\section{SUMMARY}

FY-1993 concludes eleven years of water quality monitoring in the DWPF watershed. Based on the data collected in that period several conclusions can be drawn:

1. TSS levels at all locations have risen during the sample period and have generally remained at higher levels than during the pre-construction period.

2. The small tributarieg below the construction site, Crouch Branch and McQueen Branch, contained significantly elevated TSS levels during the construction period; post-construction period effects are still evident under low and high rainfall conditions.

3. In general, during the after-construction period, levels of the water quality variables have decreased under No Rain conditions but remain elevated under High Rain conditions. This may indicate some recovery under baseflow conditions, but erosional inputs may still occur during periods of heavy rainfall.

4. Statistical evidence over the eleven year period does not reveal that the DWPF construction-site runoff has impacted UTR Creek significantly. 


\section{CONCLUSION}

In a 1986 article Kenneth L. Dickson, former president of the Society of Environmental Toxicology and Chemistry, suggested that rather than toxic chemicals, non-point pollutants such as siltation are causing the greatest harm to the aquatic environment (Dickson 1986). He states that one source of silt contamination arises from "poor erosion control practices at construction sites." Based on data from the DWPF watershed samples between 1982 and 1993 , it appears that although extensive impact to UTR Creek has been minimized, elevated TSS and other parameter levels continue to persist in tributaries below construction-site sedimentation basins. Further attention to erosion control measures, such as dredging clogged sediment basins, is recommended to improve conditions for these aquatic tributaries. 


\section{LITERATURE CITED}

Chambers, John M., W. S Cleveland, B. Kleiner, and P. A. Tukey, 1983. Graphical Methods for Data Analysis. Bell Telephone Laboratories Inc. Murray Hill, N.J. 395pp.

Dickson, Kenneth L., 1986. Neglected and forgotten contaminants affecting aquatic life. Environ. Toxicol. and Chem.5: 939-940.

Pechmann, J. H. K., R. D. Semlitsch, R. M. Lew, and D. T. Mayack, 1984. Ecological Studies Related to Construction of the Defense Waste Processing Facility on the Savannah River Plant. FY 1983-84 Annual Report. Savannah River Ecology Laboratory NTIS publ. SREL-17UC-66e, $166 \mathrm{pp}$.

Pechmann, J. H. K., D. E. Scott, H. H. MeGregor, R. A. Estes and A. C. Chazal, 1993. Ecological Studies Related to the Construction of the Defense Waste Processing Faf ity on the Savannah River Site. FY 1989-90 Annual Report. Savannah River Ecology Laboratory NTIS publ. SREL-46-UC-66e, 103pp.

SAS Institute, Inc., 1985a. SAS User's Guide: Basics, Version 5 Edition. SAS Institute Inc., Cary, N.C. 1290pp.

SAS Institute, Inc., 1985b. SAS User's Guide: Statistics, Version 5 Edition. SAS Ingtitute Inc., Cary, N.C. 956pp.

Scott, David E., J. H. K. Pechmann, J. N. Knox, R. A. Estes, and J. H. McGregor, 1988. Ecological Studies Related to the Construction of the Defense Waste Processing Facility on the Savannah River Plant. FY-1987 and FY-1988 Annual Report. Savannah River Ecology 
Laboratory. NTIS publ. SREL-43-UC-66e, $108 \mathrm{pp}$.

United States Environmental Protection Agency, 1983. Methods for chemical analysis of water and wastes. Environmental Monitoring and Support Laboratory. Cincinnati, OH. 
APPENDIX A

DWPF Water Quality Data

FY-1983 to FY-1993

(summarized by rainfall class and location) 


\begin{tabular}{|c|c|c|c|c|c|}
\hline VARIABLE & MEAN & $\begin{array}{l}\text { STD ERROR } \\
\text { OF MEAN }\end{array}$ & $\begin{array}{l}\text { MAXIMUM } \\
\text { VALUE }\end{array}$ & $\begin{array}{l}\text { MINIMUM } \\
\text { VALUE }\end{array}$ & \\
\hline TSS & 3.42 & 0.24 & 7.20 & 1.53 & 33 \\
\hline LTSS & 1.44 & 0.05 & 2.10 & 0.93 & 33 \\
\hline TURB & 6.00 & 2.00 & 8.00 & 4.00 & 2 \\
\hline LTURB & 1.90 & 0.29 & 2.20 & 1.61 & 2 \\
\hline PTSASH & 44.18 & 1.53 & 56.56 & 12.60 & 33 \\
\hline SCOND & 26.44 & 1.04 & 35.29 & 15.25 & 26 \\
\hline LSCOND & 3.29 & 0.04 & 3.59 & 2.79 & 26 \\
\hline & D RAII & LL FY-1983 & TINKER CK --- & & \\
\hline TSS & 3.82 & 0.36 & 11.14 & 1.81 & 3 \\
\hline LTSS & 1.51 & 0.06 & 2.50 & 1. & 3 \\
\hline TURB & 1.00 & & 1.00 & 1.00 & \\
\hline LTURB & 0.69 & & 0.69 & 0.69 & \\
\hline PTSASH & 42.54 & 1.52 & 52.30 & 19.40 & 3 \\
\hline SCOND & 49.55 & 2.04 & 68.64 & 32.81 & 25 \\
\hline LSCOND & 3.90 & 0.04 & 4.24 & 3.52 & 25 \\
\hline
\end{tabular}

NO RAINFALL FY-1983 MCQUEEN BR

$\begin{array}{lrrrrr}\text { TSS } & 5.38 & 0.31 & 8.56 & 3.37 & 22 \\ \text { LTSS } & 1.83 & 0.05 & 2.26 & 1.47 & 22 \\ \text { TURB } & 5.00 & 0.00 & 5.00 & 5.00 & 2 \\ \text { LTURB } & 1.79 & 0.00 & 1.79 & 1.79 & 2 \\ \text { PTSASH } & 58.64 & 1.75 & 69.86 & 34.11 & 22 \\ \text { SCOND } & 58.89 & 3.90 & 81.59 & 32.38 & 15 \\ \text { LSCOND } & 4.06 & 0.07 & 4.41 & 3.51 & 15\end{array}$

NO RAINFALL FY-1983 UTR CK MID.

$\begin{array}{lrrrrr}\text { TSS } & 4.04 & 0.42 & 8.58 & 2.02 & 17 \\ \text { LTSS } & 1.57 & 0.07 & 2.26 & 1.11 & 17 \\ \text { TURB } & 5.00 & - & 5.00 & 5.00 & 1 \\ \text { LTURB } & 1.79 & - & 1.79 & 1.79 & 1 \\ \text { PTSASH } & 47.39 & 1.13 & 52.67 & 36.60 & 15 \\ \text { SCOND } & 36.50 & 1.99 & 46.17 & 23.11 & 13 \\ \text { LSCOND } & 3.61 & 0.06 & 3.85 & 3.18 & 13\end{array}$

NO RAINFALL FY- 1983 CROUCH BR

TSS
LTSS
TU RB
LTU RB
PTSASH
SCOND
LSCOND




\begin{tabular}{lccccr} 
VARIABLE & MEAN & $\begin{array}{l}\text { STD ERROR } \\
\text { OF MEAN }\end{array}$ & $\begin{array}{l}\text { MAXIMUM } \\
\text { VALUE }\end{array}$ & $\begin{array}{l}\text { MINIMUM } \\
\text { VALUE }\end{array}$ & N \\
& & NO RAINFALL FY-1983 & UTR BR BELOW- & & \\
\hline TSS & 4.10 & 0.49 & 9.02 & 0.89 & 17 \\
LTSS & 1.56 & 0.09 & 2.30 & 0.64 & 17 \\
TURB & 5.00 & & 5.00 & 5.00 & 1 \\
LTURB & 1.79 & & 1.79 & 1.79 & 1 \\
PTSASH & 45.73 & 2.33 & 55.47 & 15.09 & 16 \\
SCOND & 36.60 & 2.05 & 47.33 & 23.96 & 14 \\
LSCOND & 3.61 & 0.06 & 3.88 & 3.22 & 14
\end{tabular}

NO RAINFALL FY-1984 UTR CK ABOVE

$\begin{array}{lrrrrr}\text { TSS } & 3.14 & 0.31 & 6.35 & 1.79 & 20 \\ \text { LTSS } & 1.37 & 0.07 & 1.99 & 1.02 & 20 \\ \text { TURB } & 2.87 & 0.41 & 7.00 & 1.00 & 18 \\ \text { LTURB } & 1.27 & 0.09 & 2.08 & 0.69 & 18 \\ \text { PTSASH } & 39.31 & 1.49 & 49.48 & 24.25 & 18 \\ \text { SCOND } & 13.14 & 2.19 & 30.48 & 1.26 & 20 \\ \text { LSCOND } & 2.39 & 0.17 & 3.45 & 0.81 & 20\end{array}$

NO RAINFALL FY-1984 TINKER CK

$\begin{array}{lrrrrr}\text { TSS } & 3.50 & 0.43 & 6.65 & 1.32 & 18 \\ \text { LTSS } & 1.43 & 0.09 & 2.04 & 0.84 & 18 \\ \text { TURB } & 4.62 & 1.54 & 27.00 & 1.00 & 16 \\ \text { LTURB } & 1.48 & 0.15 & 3.33 & 0.69 & 16 \\ \text { PTSASH } & 39.32 & 2.11 & 54.40 & 24.20 & 16 \\ \text { SCOND } & 13.96 & 2.10 & 31.34 & 1.54 & 18 \\ \text { LSCOND } & 2.48 & 0.18 & 3.48 & 0.93 & 18\end{array}$

NO RAINFALL FY-1984 MCQUEEN BR

\begin{tabular}{lrrrrr}
\hline TSS & 27.69 & 11.26 & 233.82 & 2.74 & 20 \\
LTSS & 2.75 & 0.22 & 5.46 & 1.32 & 20 \\
TURB & 41.67 & 21.35 & 390.00 & 1.00 & 18 \\
LTURB & 2.66 & 0.34 & 5.97 & 0.69 & 18 \\
PTSASH & 72.51 & 1.70 & 85.02 & 58.12 & 18 \\
SCOND & 36.30 & 14.28 & 305.50 & 6.02 & 20 \\
LSCOND & 3.20 & 0.16 & 5.73 & 1.95 & 20 \\
& & & & & \\
\hline TSS & NO RAINFALL & FY-1984 & UTR CK MID. & & \\
LTSS & 4.77 & 0.84 & 9.52 & 2.01 & 10 \\
TURB & 1.67 & 0.14 & 2.35 & 1.10 & 10 \\
LTURB & 3.78 & 0.87 & 9.00 & 0.00 & 9 \\
PTSASH & 1.40 & 0.23 & 2.30 & 0.00 & 9 \\
SCOND & 45.02 & 2.15 & 53.11 & 38.71 & 9 \\
LSCOND & 14.74 & 3.49 & 40.18 & 1.50 & 10 \\
& 2.52 & 0.24 & 3.72 & 0.91 & 10
\end{tabular}


VARIABLE

$\begin{array}{llll} & \text { STD ERROR } & \text { MAXIMUM } & \text { MINIMUM } \\ \text { MEAN } & \text { OF MEAN } & \text { VALUE } & \text { VALUE }\end{array}$

$\mathbf{N}$

NO RAINFALL FY-1984 CROUCH BR

TSS
LTSS
TURB
LTURB
PTSASH
SCOND
LSCOND

--

NO RAINFALL FY-1984

$\begin{array}{lrr}\text { TSS } & 4.95 & 0.75 \\ \text { LTSS } & 1.72 & 0.12 \\ \text { TURB } & 4.01 & 0.53 \\ \text { LTURB } & 1.56 & 0.12 \\ \text { PTSASH } & 42.90 & 2.77 \\ \text { SCOND } & 14.85 & 2.22 \\ \text { LSCOND } & 2.62 & 0.21\end{array}$

NO RAINFALL FY-1985

TSS

6.83

LTSS

TURB

1.83

3.49

1.57

LTURB

1.40

0.17

0.63

PTSASH

42.55

SCOND

19.71

0.11

3.00

3.45

1.64

0.07

UTR BR BELOW

LSCOND

NO RAINFALL FY- 1985

TSS
LTSS
TURB
LTURB
PTSASH
SCOND
LSCOND
6.84
1.86
1.40
3.72
0.14
1.45
0.54
48.20
0.10
25.97
1.71
3.21
2.41
0.13

8.82

$2.86 \quad 10$

2.28

7.00

1.35

2.08

58.28

29.32

3.41

1.00

10

0.69

9

34.38

9

1.49

10

0.91

10

UTR CK ABOVE

$\begin{array}{rrr}\text { K ABOVE } & & \\ 24.90 & 1.30 & 16 \\ 3.25 & 0.83 & 16 \\ 11.50 & 1.50 & 16 \\ 2.53 & 0.92 & 16 \\ 57.00 & 12.87 & 16 \\ 39.37 & 11.85 & 15 \\ 3.70 & 2.55 & 15\end{array}$

NO RAINFALL FY-1985

$\begin{array}{lrr}\text { TSS } & 8.13 & 0.83 \\ \text { LTSS } & 2.17 & 0.09 \\ \text { TURB } & 12.43 & 2.77 \\ \text { LTURB } & 2.44 & 0.16 \\ \text { PTSASH } & 69.84 & 1.62 \\ \text { SCOND } & 58.18 & 8.88 \\ \text { LSCOND } & 3.99 & 0.14\end{array}$

MCQUEEN BR

$\begin{array}{rrr}27.60 & 1.40 & 19 \\ 3.35 & 0.88 & 19 \\ 10.80 & 1.20 & 19 \\ 2.47 & 0.79 & 19 \\ 59.00 & 32.77 & 19 \\ 34.97 & 5.93 & 14 \\ 3.58 & 1.94 & 14\end{array}$

12.18

2.58

32.00

3.50

79.30

121.23

4.81
4.70

1.74

5.20

1.82

60.73

22.73

3.17
11

11

11

11

11

10 


\begin{tabular}{lcr} 
VARIABLE & MEAN & $\begin{array}{r}\text { STD ERRO } \\
\text { OF MEAN }\end{array}$ \\
& & \\
\hline TSS & NO RAINFALL & FY-1985 \\
LTSS & 6.49 & 0.99 \\
TURB & 1.94 & 0.13 \\
LTURB & 3.92 & 0.53 \\
PTSASH & 1.55 & 0.10 \\
SCOND & 48.33 & 3.14 \\
LSCOND & 19.45 & 0.84 \\
& 3.01 & 0.05
\end{tabular}

NO RAINFALL FY-1985

$\begin{array}{lrr}\text { TSS } & 35.00 & 17.71 \\ \text { LTSS } & 3.34 & 0.48 \\ \text { TURB } & 79.77 & 45.59 \\ \text { LTURB } & 4.06 & 0.57 \\ \text { PTSASH } & 85.43 & 1.50 \\ \text { SCOND } & 75.26 & 8.21 \\ \text { LSCOND } & 4.36 & 0.11\end{array}$

NO RAINFALL FY-1985

$\begin{array}{lcc}\text { TSS } & 5.79 & 0.78 \\ \text { LTSS } & 1.86 & 0.11 \\ \text { TURB } & 4.21 & 0.60 \\ \text { LTURB } & 1.60 & 0.11 \\ \text { PTSASH } & 50.19 & 1.36 \\ \text { SCOND } & 20.79 & 0.99 \\ \text { LSCOND } & 3.07 & 0.05\end{array}$

NO RAINFALL FY-1986

TSS

LTSS

TURB

LTURB

PTSASH

SCOND

LSCOND
8.93

2.01

4.03

1.45

54.76

16.99

2.89
2.39

0.16

0.96

0.12

1.21

0.39

0.02
MAXIMUM

VALUE

UTR CK MID.

13.00

2.64

6.90

2.07

57.00

21.93

3.13

CROUCH BR

$$
\begin{array}{r}
70.00 \\
4.26
\end{array}
$$

170.00

5.14

88.40

92.26

4.54

UTR CK BELOW

$$
\begin{array}{r}
9.50 \\
2.35 \\
7.80 \\
2.17 \\
58.00 \\
24.87 \\
3.25
\end{array}
$$

MINIMUM

VALUE

$\mathbf{N}$

$\begin{array}{rr}2.60 & 10 \\ 1.28 & 10 \\ 2.10 & 10 \\ 1.13 & 10 \\ 25.09 & 10 \\ 13.76 & 9 \\ 2.69 & 9\end{array}$

$\begin{array}{rrr}9.50 & 3.10 & 10 \\ 2.35 & 1.41 & 10 \\ 7.80 & 2.60 & 10 \\ 2.17 & 1.28 & 10 \\ 58.00 & 43.80 & 10 \\ 24.87 & 14.90 & 9 \\ 3.25 & 2.77 & 9\end{array}$

UTR CK ABOVE

43.90

3.80

19.00

2.99

12.80

3

2.623

$23.30 \quad 3$

3.193

$83.60 \quad 3$

$63.83 \quad 3$

4.17

3

NO RAINFALL FY-1986

$\begin{array}{lrr}\text { TSS } & 6.37 & 1.38 \\ \text { LTSS } & 1.82 & 0.14 \\ \text { TURB } & 3.22 & 0.49 \\ \text { LTURB } & 1.36 & 0.10 \\ \text { PTSASH } & 50.28 & 1.90 \\ \text { SCOND } & 33.21 & 1.01 \\ \text { LSCOND } & 3.53 & 0.03\end{array}$

66.70

20.30

3.06

$2.90 \quad 18$

$1.36 \quad 18$

$1.30 \quad 18$

$0.83 \quad 18$

$44.80 \quad 18$

$14.40 \quad 18$

$2.73 \quad 18$

TINKER CK

$\begin{array}{rrr}25.60 & 2.20 & 17 \\ 3.28 & 1.16 & 17 \\ 9.70 & 0.90 & 17 \\ 2.37 & 0.64 & 17 \\ 56.70 & 30.80 & 17 \\ 41.70 & 26.70 & 17 \\ 3.75 & 3.32 & 17\end{array}$




\begin{tabular}{lrr} 
VARIABLE & MEAN & \multicolumn{1}{c}{$\begin{array}{l}\text { STD ERRO } \\
\text { OF MEAN }\end{array}$} \\
& & \\
\hline TSS & 16.02 & 3.57 \\
LTSS & 2.67 & 0.20 \\
TURB & 19.89 & 6.20 \\
LTURB & 2.71 & 0.28 \\
PTSASH & 78.86 & 2.37 \\
SCOND & 85.50 & 9.91 \\
LSCOND & 4.41 & 0.11
\end{tabular}

NO RAINFALL FY-1986
2.09

0.21

1.17

0.18

2.68

1.00

0.04
TSS

LTSS

TURB

LTURB

PTSASH

SCOND

LSCOND

8.33

2.07

4.72

1.61

55.48

23.82

3.21

NO RAINFALL FY-1986

TSS

LTSS

TURB

LTURB

PTSASH

SCOND

LSCOND 105.13

4.28

177.28

4.79

86.40

97.33

4.58

MAXIMUM

VALUE

MINIMUM

VALUE

$\mathbf{N}$

MCQUEEN BR

$\begin{array}{rrr}35.50 & 5.10 & 9 \\ 3.60 & 1.81 & 9 \\ 56.00 & 6.40 & 9 \\ 4.04 & 2.00 & 9 \\ 89.00 & 65.70 & 9 \\ 133.20 & 54.00 & 9 \\ 4.90 & 4.01 & 9\end{array}$

UTR CK MID.

19.20

3.01

3.10

1.41

1.40

13.00

0.88

2.64

44.40

68.40

30.00

3.43

20.80

3.08

CROUCH BR

$\begin{array}{rrr}217.10 & 16.10 & 9 \\ 5.38 & 2.84 & 9 \\ 365.00 & 19.00 & 9 \\ 5.90 & 2.99 & 9 \\ 90.00 & 76.40 & 9 \\ 125.20 & 76.70 & 9 \\ 4.84 & 4.35 & 9\end{array}$

NO RAINFALL FY-1986

TSS

LTSS

TURB

LTURB

PTSASH

SCOND

LSCOND

$\begin{array}{rl}7.04 & 1.33 \\ 1.99 & 0.15 \\ 4.60 & 1.09 \\ 1.61 & 0.16 \\ 54.24 & 2.59 \\ 24.68 & 1.24 \\ 3.24 & 0.04\end{array}$

NO RAINFALL FY-1987

TSS

LTSS

TURB

LTURB

PTSASH

SCOND

LSCOND
5.00

1.76

2.70

1.19

52.68

16.85

2.87
0.61

0.10

0.77

0.18

1.67

0.93

0.05
UTR CK BELOW

14.30
2.73
12.00
2.56
72.10
33.20
3.53

4.30
2.73

$3.30 \quad 9$

1.469

$2.20 \quad 9$

1.169

$45.30 \quad 9$

$21.70 \quad 9$

3.12

UTR CK ABOVE

$\begin{array}{rrr}7.80 & 3.20 & 8 \\ 2.17 & 1.44 & 8 \\ 7.30 & 0.90 & 8 \\ 2.12 & 0.64 & 8 \\ 61.50 & 45.50 & 8 \\ 21.70 & 14.60 & 8 \\ 3.12 & 2.75 & 8\end{array}$




\section{VARIABLE}

\section{MEAN}

STD ERROR

NO RAINFALL FY-1987

$\begin{array}{lrr}\text { TSS } & 4.36 & 0.45 \\ \text { LTSS } & 1.65 & 0.08 \\ \text { TURB } & 2.21 & 0.29 \\ \text { LTURB } & 1.14 & 0.10 \\ \text { PTSASH } & 51.21 & 1.90 \\ \text { SCOND } & 28.86 & 1.55 \\ \text { LSCOND } & 3.39 & 0.05\end{array}$

NO RAINFALL FY-1987

TSS

LTSS

TURB

LTURB

PTSASH

SCOND

LSCOND

11.13

2.37

14.13

2.41

80.60

68.90

4.21
2.36

0.19

4.86

0.28

1.96

6.73

0.10
NO RAINFALL FY-1987

TSS
LTSS
TURB
LTURB
PTSASH
SCOND
LSCOND

5.20

1.80

2.98

1.31

57.38

22.60

3.16

NO RAINFALL

91.50

3.91

LTSS

TURB

LTURB

PTSASH

SCOND

LSCOND
179.50

4.44

86.17

96.57

4.58
0.74

0.12

0.89

0.22

1.42

1.07

0.05
MAXIMUM

VALUE

TINKER CR

$\begin{array}{rrr}6.10 & 2.80 & 8 \\ 1.96 & 1.34 & 8 \\ 3.40 & 1.00 & 8 \\ 1.48 & 0.69 & 8 \\ 58.30 & 42.90 & 8 \\ 35.50 & 24.30 & 8 \\ 3.59 & 3.23 & 8\end{array}$

MCQUEEN BR

22.00
3.14
40.50
3.73
85.00
101.50
4.63

UTR CK MID.

6.80

2.05

5.40

1.86

60.00

24.70

3.25

MINIMUM

VALUE

$\mathbf{N}$ 
50

VARIABLE

MEAN

STD ERROR

MAXIMUM

VALUE

MINIMUM

OF MEAN

VALUE

$\mathbf{N}$

NO RAINFALL FY-1988 UTR CK ABOVE

$\begin{array}{lrr}\text { TSS } & 4.27 & 0.86 \\ \text { LTSS } & 1.60 & 0.16 \\ \text { TU RB } & 1.37 & 0.23 \\ \text { LTURB } & 0.84 & 0.10 \\ \text { PTSASH } & 53.30 & 1.40 \\ \text { SCOND } & 14.70 & 0.33 \\ \text { LSCOND } & 2.75 & 0.02\end{array}$

NO RAINFALL FY-1988

TSS

7.82

2.30

LTSS

2.01

0.25

TURB

2.63

0.59

LTURB

1.21

0.18

PTSASH

57.08

2.99

SCOND

31.82

1.53

LSCOND

3.49

0.05

7.90

2.19

2.106

2.20

1.136

1.16

0.706

57.40

16.10

0.53

47.60

14.006

2.84

2.716

TINKER CK

$\begin{array}{rrr}17.50 & 2.50 & 6 \\ 2.92 & 1.25 & 6 \\ 4.50 & 0.80 & 6 \\ 1.70 & 0.59 & 6 \\ 71.70 & 52.00 & 6 \\ 36.10 & 27.70 & 6 \\ 3.61 & 3.36 & 6\end{array}$

NO RAINFALL FY-1988

TSS

LTSS

TURB

LTURB

PTSASH

SCOND

LSCOND
5.80

1.82

6.90

1.77

75.37

137.72

4.71

NO RAINFALL

\section{TSS}

LTSS

TURB

LTURB

PTSASH

SCOND

LSCOND
5.73

1.87

2.00

1.07

48.07

20.80

3.08
1.36

0.20

3.02

0.32

3.14

46.21

0.28

MCQUEEN BR

10.30
2.42
21.10
3.09
85.40
349.00
5.86

2.106

1.136

1.706

0.996

66.706

$58.10 \quad 6$

4.086

NO RAINFALL FY-1988

TSS

LTSS

TURB

LTURB

PTSASH

SCOND

LSCOND
13.60

2.57

21.37

2.90

78.10

106.30

4.66
4.17

0.36

8.64

0.49

2.42

12.23

0.12

UTR CK MID.

7.60

2.15

2.70

1.31

55.60

22.90

3.17
3.303

1.463

1.003

0.693

$33.30 \quad 3$

$19.40 \quad 3$

3.013

CROUCH BR

18.40

2.97

36.00

3.61

81.90

120.00

4.80
5.303

1.843

6.103

1.963

73.603

$81.90 \quad 3$

4.423 
VARIABLE

TSS

LTSS

TURB

LTURB

PTSASH

SCOND

LSCOND
MEAN

NO RAINFALL FY-1988

$\begin{array}{rr}5.43 & 0.87 \\ 1.84 & 0.15 \\ 1.90 & 0.47 \\ 1.04 & 0.18 \\ 53.80 & 2.66 \\ 21.47 & 1.08 \\ 3.11 & 0.05\end{array}$

NO RAINFALL FY-1989

$\begin{array}{lrr}\text { TSS } & 3.60 & 0.43 \\ \text { LTSS } & 1.50 & 0.10 \\ \text { TURB } & 0.73 & 0.26 \\ \text { LTURB } & 0.50 & 0.13 \\ \text { PTSASH } & 49.87 & 1.71 \\ \text { SCOND } & 14.92 & 0.33 \\ \text { LSCOND } & 2.77 & 0.02\end{array}$

NO RAINFALL FY-1989

$\begin{array}{lrr}\text { TSS } & 5.67 & 1.63 \\ \text { LTSS } & 1.76 & 0.23 \\ \text { TURB } & 1.63 & 0.55 \\ \text { LTURB } & 0.85 & 0.21 \\ \text { PTSASH } & 52.52 & 2.48 \\ \text { SCOND } & 30.68 & 1.44 \\ \text { LSCOND } & 3.45 & 0.05\end{array}$

NO RAINFALL FY-1989

$\begin{array}{lrr}\text { TSS } & 5.25 & 1.13 \\ \text { LTSS } & 1.75 & 0.18 \\ \text { TURB } & 6.90 & 2.41 \\ \text { LTURB } & 1.87 & 0.27 \\ \text { PTSASH } & 76.90 & 2.04 \\ \text { SCOND } & 92.83 & 24.14 \\ \text { LSCOND } & 4.39 & 0.24\end{array}$

NO RAINFALL FY-1989

TSS

LTSS

TURB

LTURB

PTSASH

SCOND

LSCOND

\begin{abstract}
4.23
\end{abstract}
1.62

1.07

0.66

53.03

22.17

3.14
0.94

0.18

0.57

$0: 26$

0.67

1.62

0.07
UTR CK BELOW

6.50
2.01
2.60
1.28
57.40
23.50
3.20

3.703

1.553

1.003

0.693

48.603

$19.80 \quad 3$

3.033

UTR CK ABOVE

$\begin{array}{rrr}\text { ABOVE } & & \\ 4.50 & 2.10 & 6 \\ 1.70 & 1.13 & 6 \\ 2.00 & 0.32 & 6 \\ 1.10 & 0.28 & 6 \\ 57.50 & 46.50 & 6 \\ 16.40 & 14.20 & 6 \\ 2.86 & 2.72 & 6\end{array}$

TINKER CK

$\begin{array}{rcc}\text { CK } & & \\ 12.50 & 1.90 & 6 \\ 2.60 & 1.06 & 6 \\ 3.30 & 0.38 & 6 \\ 1.46 & 0.32 & 6 \\ 60.00 & 43.2 & 6 \\ 34.80 & 27.10 & 6 \\ 3.58 & 3.34 & 6\end{array}$

MCQUEEN BR

9.10

2.31

18.00

2.94

83.50

180.00

5.20

$3.34 \quad 6$

MAXIMUM MINIMUM

VALUE N

$\mathbf{N}$


52

\begin{tabular}{lcr} 
VARIABLE & MEAN & $\begin{array}{r}\text { STD ERROR } \\
\text { OF MEAN }\end{array}$ \\
& & \\
\hline TSS & 32.93 & 14.33 \\
LTSS & 3.22 & 0.63 \\
TURB & 66.63 & 30.87 \\
LTURB & 3.73 & 0.84 \\
PTSASH & 86.13 & 1.88 \\
SCOND & 99.60 & 4.33 \\
LSCOND & 4.61 & 0.04
\end{tabular}

NO RAINFALL FY-1989

$\begin{array}{lrr}\text { TSS } & 5.33 & 1.39 \\ \text { LTSS } & 1.79 & 0.25 \\ \text { TURB } & 1.48 & 0.92 \\ \text { LTURB } & 0.78 & 0.34 \\ \text { PTSASH } & 58.80 & 3.05 \\ \text { SCOND } & 23.17 & 1.72 \\ \text { LSCOND } & 3.18 & 0.07\end{array}$

NO RAINFALL FY-1990

$\begin{array}{lrr}\text { TSS } & 4.85 & 0.49 \\ \text { LTSS } & 1.75 & 0.09 \\ \text { TURB } & 1.03 & 0.19 \\ \text { LTURB } & 0.69 & 0.09 \\ \text { PTSASH } & 55.92 & 1.24 \\ \text { SCOND } & 15.30 & 0.35 \\ \text { LSCOND } & 2.79 & 0.02\end{array}$

NO RAINFALL FY-1990

$\begin{array}{lrr}\text { TSS } & 9.93 & 0.93 \\ \text { LTSS } & 2.37 & 0.09 \\ \text { TURB } & 2.32 & 0.32 \\ \text { LTURB } & 1.17 & 0.13 \\ \text { PTSASH } & 52.18 & 2.74 \\ \text { SCOND } & 32.28 & 1.72 \\ \text { LSCOND } & 3.50 & 0.05\end{array}$

NO RAINFALL FY-1990

$\begin{array}{lrr}\text { TSS } & 3.92 & 0.48 \\ \text { LTSS } & 1.57 & 0.09 \\ \text { TURB } & 3.02 & 0.31 \\ \text { LTURB } & 1.38 & 0.08 \\ \text { PTSASH } & 75.58 & 1.75 \\ \text { SCOND } & 54.82 & 4.54 \\ \text { LSCOND } & 4.01 & 0.08\end{array}$

CROUCH BR

MAXIMUM MINIMUM

VALUE VALUE N

$\begin{array}{rrr}55.40 & 6.30 & 3 \\ 4.03 & 1.99 & 3 \\ 110.00 & 6.90 & 3 \\ 4.71 & 2.07 & 3 \\ 88.80 & 82.50 & 3 \\ 107.00 & 92.00 & 3 \\ 4.68 & 4.53 & 3\end{array}$

UTR CK BELOW

$\begin{array}{rrr}7.40 & 2.70 & 3 \\ 2.13 & 1.31 & 3 \\ 3.30 & 0.43 & 3 \\ 1.46 & 0.36 & 3 \\ 64.90 & 55.60 & 3 \\ 26.60 & 21.30 & 3 \\ 3.32 & 3.10 & 3\end{array}$

UTR CK ABOVE

$\begin{array}{rrr}\text { ABOVE } & & \\ 6.40 & 3.30 & 6 \\ 2.00 & 1.46 & 6 \\ 1.60 & 0.52 & 6 \\ 0.96 & 0.42 & 6 \\ 60.90 & 52.40 & 6 \\ 16.40 & 14.40 & 6 \\ 2.86 & 2.73 & 6\end{array}$

TINKER CK

$\begin{array}{rrr}12.20 & 6.40 & 6 \\ 2.58 & 2.00 & 6 \\ 2.80 & 0.72 & 6 \\ 1.34 & 0.54 & 6 \\ 56.70 & 39.30 & 6 \\ 39.30 & 27.70 & 6 \\ 3.70 & 3.36 & 6\end{array}$

MCQUEEN BR

5.90

1.93

2.506

1.256

4.20

2.006

1.65

1.106

81.80

70.506

75.10

45.406

4.33

3.846 
53

VARIABLE

MEAN

STD ERROR

OF MEAN

MAXIMUM

VALUE

MINIMUM

VALUE

$\mathbf{N}$

NO RAINFALL FY-1990 UTR CK MID.

$\begin{array}{lrr}\text { TSS } & 6.87 & 0.44 \\ \text { LTSS } & 2.06 & 0.06 \\ \text { TURB } & 1.68 & 0.42 \\ \text { LTURB } & 0.96 & 0.17 \\ \text { PTSASH } & 55.13 & 1.51 \\ \text { SCOND } & 20.73 & 0.63 \\ \text { LSCOND } & 3.08 & 0.03\end{array}$

7.40

2.12

2.20

1.16

58.10

21.60

3.12

6.00

1.95

0.84

0.61

53.20

19.50

3.02

NO RAINFALL FY-1990

TSS

LTSS

TURB

LTURB

PTSASH

SCOND

LSCOND
27.00

3.02

29.97

3.02

74.13

130.63

4.78
15.82

0.55

14.76

0.74

5.48

42.14

0.32

CROUCH BR

$\begin{array}{rrr}58.50 & 8.70 & 3 \\ 4.09 & 2.27 & 3 \\ 55.00 & 3.90 & 3 \\ 4.02 & 1.59 & 3 \\ 80.30 & 63.20 & 3 \\ 212.00 & 70.90 & 3 \\ 5.36 & 4.26 & 3\end{array}$

NO RAINFALL FY-1990 UTR CK BELOW

$\begin{array}{lcc}\text { TSS } & 8.47 & 1.82 \\ \text { LTSS } & 2.21 & 0.18 \\ \text { TURB } & 1.26 & 0.47 \\ \text { LTURB } & 0.78 & 0.20 \\ \text { PTSASH } & 57.23 & 0.67 \\ \text { SCOND } & 21.77 & 0.54 \\ \text { LSCOND } & 3.12 & 0.02\end{array}$

12.10

2.57

2.20

1.16

58.50

22.40

3.15

6.60

2.03

3

0.71

0.54

56.20

20.70

3.08

NO RAINFALL FY-1991 UTR CK ABOVE

$\begin{array}{lrr}\text { TSS } & 2.91 & 0.10 \\ \text { LTSS } & 1.36 & 0.03 \\ \text { TURB } & 0.52 & 0.01 \\ \text { LTURB } & 0.42 & 0.01 \\ \text { PTSASH } & 49.55 & 2.56 \\ \text { SCOND } & 15.18 & 0.23 \\ \text { LSCOND } & 2.78 & 0.01\end{array}$

3.16

1.43

0.55

0.44

54.60

15.70

2.82

$\begin{array}{rr}2.71 & 4 \\ 1.31 & 4 \\ 0.48 & 4 \\ 0.39 & 4 \\ 43.70 & 4 \\ 14.70 & 4 \\ 2.75 & 4\end{array}$

NO RAINFALL FY-1991 TINKER CK

$\begin{array}{lrrrrr}\text { TSS } & 2.79 & 0.35 & 3.47 & 1.91 & 4 \\ \text { LTSS } & 1.32 & 0.10 & 1.50 & 1.07 & 4 \\ \text { TURB } & 0.54 & 0.06 & 0.67 & 0.40 & 4 \\ \text { LTURB } & 0.43 & 0.04 & 0.51 & 0.34 & 4 \\ \text { PTSASH } & 51.33 & 2.48 & 58.10 & 46.60 & 4 \\ \text { SCOND } & 29.25 & 1.30 & 32.80 & 26.70 & 4 \\ \text { LSCOND } & 3.41 & 0.04 & 3.52 & 3.32 & 4\end{array}$


54

VARIABLE

MEAN

STD ERROR

OF MEAN

MAXIMUM

MINIMUM

VALUE

VALUE

$\mathbf{N}$

NO RAINFALL FY-1991

$\begin{array}{lrr}\text { TSS } & 3.29 & 0.24 \\ \text { LTSS } & 1.45 & 0.06 \\ \text { TURB } & 1.24 & 0.12 \\ \text { LTURB } & 0.80 & 0.06 \\ \text { PTSASH } & 71.65 & 1.90 \\ \text { SCOND } & 39.65 & 0.82 \\ \text { LSCOND } & 3.70 & 0.02\end{array}$

MCQUEEN BR

NO RAINFALL FY-1991 UTR CK MID.

$\begin{array}{lrrrrr}\text { TSS } & 3.29 & 0.11 & 3.40 & 3.18 & 2 \\ \text { LTSS } & 1.46 & 0.03 & 1.48 & 1.43 & 2 \\ \text { TURB } & 0.56 & 0.07 & 0.62 & 0.49 & 2 \\ \text { LTURB } & 0.44 & 0.04 & 0.48 & 0.40 & 2 \\ \text { PTSASH } & 47.65 & 3.25 & 50.90 & 44.40 & 2 \\ \text { SCOND } & 20.95 & 0.85 & 21.80 & 20.10 & 2 \\ \text { LSCOND } & 3.09 & 0.04 & 3.13 & 3.05 & 2\end{array}$

NO RAINFALL FY- 1991 CROUCH BR

$\begin{array}{lrrrrr}\text { TSS } & 11.83 & 1.81 & 13.63 & 10.02 & 2 \\ \text { LTSS } & 2.54 & 0.14 & 2.68 & 2.40 & 2 \\ \text { TURB } & 5.63 & 2.58 & 8.20 & 3.05 & 2 \\ \text { LTURB } & 1.81 & 0.41 & 2.22 & 1.40 & 2 \\ \text { PTSASH } & 75.50 & 7.00 & 82.50 & 68.50 & 2 \\ \text { SCOND } & 76.95 & 4.85 & 81.80 & 72.10 & 2 \\ \text { LSCOND } & 4.35 & 0.06 & 4.42 & 4.29 & 2\end{array}$

NO RAINFALL FY-1991 UTR CK BELOW-

$\begin{array}{lrrrrr}\text { TSS } & 4.10 & 0.16 & 4.25 & 3.94 & 2 \\ \text { LTSS } & 1.63 & 0.03 & 1.66 & 1.60 & 2 \\ \text { TURB } & 0.66 & 0.07 & 0.72 & 0.59 & 2 \\ \text { LTURB } & 0.50 & 0.04 & 0.54 & 0.46 & 2 \\ \text { PTSASH } & 55.50 & 0.90 & 56.40 & 54.60 & 2 \\ \text { SCOND } & 21.15 & 0.65 & 21.80 & 20.50 & 2 \\ \text { LSCOND } & 3.10 & 0.03 & 3.13 & 3.07 & 2\end{array}$

NO RAINFALL FY-1992 UTR CK ABOVE

$\begin{array}{lr}\text { TSS } & 3.71 \\ \text { LTSS } & 1.54 \\ \text { TURB } & 0.44 \\ \text { LTURB } & 0.36 \\ \text { PTSASH } & 41.85 \\ \text { SCOND } & 15.15 \\ \text { LSCOND } & 2.78\end{array}$

0.46

0.10

0.04

4.17

3.25

2

1.64

1.45

0.47

0.40

0.39

0.34

43.40

1.55

0.15

15.30

40.30

15.00

2.79

2.77

0.01 
VARIABLE

MEAN

STD ERROR
OF MEAN

MAXIMUM

MINIMUM

VALUE

VALUE

$\mathbf{N}$

-NO RAINFALL FY-1992 TINKER CK

TSS
LTSS
TURB
LTURB
PTSAS
SCOND
LSCOND

4.55

1.71

0.53

0.42

$\mathrm{H} 47.6 \mathrm{O}$

25.40

3.27
0.43

0.08

0.02

0.01

0.50

2.20

0.08
4.97

1.79

0.54

0.43

48.10

27.60

3.35
4.12

1.63

0.51

0.41

47.10

23.20

3.19

\section{2}

2

2

2

2

2

NO RAINFALL FY-1992 MCQUEEN BR

TSS
LTSS
TURB
LTURB
PTSASH
SCOND
LSCOND

$\begin{array}{rl}4.12 & 0.67 \\ 1.62 & 0.13 \\ 1.28 & 0.42 \\ 0.81 & 0.19 \\ 55.95 & 0.85 \\ 53.30 & 7.50 \\ 3.98 & 0.14\end{array}$

0.67

0.13

4.79

3.45

1.49

1.76

0.86

1.70

0.62

0.99

56.80

55.10

60.80

4.12

45.80

3.85

2

NO RAINFALL FY-1992 UTR CK MID.

TSS
LTSS
TURB
LTURB
PTSASH
SCOND
LSCOND

4.00
1.61
0.46
0.38
39.00
20.40
3.06

--
--
--
--
--
--

$\begin{array}{rrr}4.00 & 4.00 & 1 \\ 1.61 & 1.61 & 1 \\ 0.46 & 0.46 & 1 \\ 0.38 & 0.38 & 1 \\ 39.00 & 39.00 & 1 \\ 20.40 & 20.40 & 1 \\ 3.06 & 3.06 & 1\end{array}$

NO RAINFALL FY-1992

$\begin{array}{lr}\text { TSS } & 20.27 \\ \text { LTSS } & 3.06 \\ \text { TURB } & 39.00 \\ \text { LTURB } & 3.69 \\ \text { PTSASH } & 78.60 \\ \text { SCOND } & 78.20 \\ \text { LSCOND } & 4.37\end{array}$

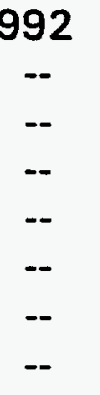

CROUCH BR

$\begin{array}{rrr}\text { BR } & \\ 20.27 & 20.27 & 1 \\ 3.06 & 3.06 & 1 \\ 39.00 & 39.00 & 1 \\ 3.69 & 3.69 & 1 \\ 78.60 & 78.60 & 1 \\ 78.20 & 78.20 & 1 \\ 4.37 & 4.37 & 1\end{array}$

NO RAINFALL FY-1992

$\begin{array}{lr}\text { TSS } & 4.51 \\ \text { LTSS } & 1.71 \\ \text { TURB } & 0.51 \\ \text { LTURB } & 0.41 \\ \text { PTSASH } & 48.60 \\ \text { SCOND } & 20.80 \\ \text { LSCOND } & 3.08\end{array}$

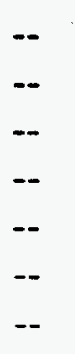

UTR CK BELOW

$\begin{array}{rrr}4.51 & 4.51 & 1 \\ 1.71 & 1.71 & 1 \\ 0.51 & 0.51 & 1 \\ 0.41 & 0.41 & 1 \\ 48.60 & 48.60 & 1 \\ 20.80 & 20.80 & 1 \\ 3.08 & 3.08 & 1\end{array}$


VARIABLE

MEAN

STD ERROR MAXIMUM MINIMUM OF MEAN VALUE VALUE N

-NO RAINFALL FY-1993 UTR CK ABOVE

$\begin{array}{lrrrrr}\text { TSS } & 6.19 & 0.81 & 9.97 & 3.17 & 8 \\ \text { LTSS } & 1.93 & 0.11 & 2.40 & 1.43 & 8 \\ \text { TURB } & 4.58 & 0.45 & 6.70 & 3.40 & 8 \\ \text { LTURB } & 1.70 & 0.08 & 2.04 & 1.41 & 8 \\ \text { PTSASH } & 63.21 & 3.58 & 83.90 & 55.00 & 8 \\ \text { SCOND } & 13.85 & 0.07 & 14.10 & 13.60 & 8 \\ \text { LSCOND } & 2.70 & 0.01 & 2.71 & 2.68 & 8\end{array}$

NO RAINFALL FY-1993 TINKER CK

$\begin{array}{lrrrrr}\text { TSS } & 6.94 & 1.13 & 13.19 & 3.45 & 8 \\ \text { LTSS } & 2.00 & 0.14 & 2.65 & 1.49 & 8 \\ \text { TURB } & 5.19 & 0.49 & 7.80 & 3.60 & 8 \\ \text { LTURB } & 1.80 & 0.08 & 2.17 & 1.53 & 8 \\ \text { PTSAS } & 55.69 & 3.10 & 74.90 & 49.00 & 8 \\ \text { SCOND } & 24.64 & 0.95 & 28.70 & 21.40 & 8 \\ \text { LSCOND } & 3.24 & 0.04 & 3.39 & 3.11 & 8\end{array}$

NO RAINFALL FY-1993 MCQUEEN BR

$\begin{array}{lrrrrr}\text { TSS } & 9.43 & 3.14 & 28.07 & 3.13 & 8 \\ \text { LTSS } & 2.11 & 0.24 & 3.37 & 1.42 & 8 \\ \text { TURB } & 6.09 & 0.80 & 11.00 & 4.30 & 8 \\ \text { LTURB } & 1.92 & 0.10 & 2.48 & 1.67 & 8 \\ \text { PTSASH } & 69.61 & 2.58 & 82.40 & 60.50 & 8 \\ \text { SCOND } & 41.63 & 3.93 & 60.10 & 30.80 & 8 \\ \text { LSCOND } & 3.72 & 0.09 & 4.11 & 3.46 & 8\end{array}$

NO RAINFALL FY-1993 UTR CK MID.

$\begin{array}{lrrrrr}\text { TSS } & 7.93 & 1.49 & 11.17 & 5.33 & 4 \\ \text { LTSS } & 2.15 & 0.17 & 2.50 & 1.85 & 4 \\ \text { TURB } & 4.95 & 0.55 & 6.40 & 3.80 & 4 \\ \text { LTURB } & 1.77 & 0.09 & 2.00 & 1.57 & 4 \\ \text { PTSASH } & 58.33 & 2.04 & 64.00 & 54.30 & 4 \\ \text { SCOND } & 18.80 & 0.94 & 21.60 & 17.60 & 4 \\ \text { LSCOND } & 2.98 & 0.05 & 3.12 & 2.92 & 4\end{array}$

NO RAINFALL FY-1993 CROUCH BR

$\begin{array}{lrrrrr}\text { TSS } & 10.42 & 2.12 & 16.01 & 6.17 & 4 \\ \text { LTSS } & 2.38 & 0.18 & 2.83 & 1.97 & 4 \\ \text { TURB } & 18.10 & 4.42 & 27.00 & 8.40 & 4 \\ \text { LTURB } & 2.86 & 0.26 & 3.33 & 2.24 & 4 \\ \text { PTSASH } & 82.48 & 4.50 & 95.90 & 76.70 & 4 \\ \text { SCOND } & 89.02 & 21.04 & 130.70 & 51.20 & 4 \\ \text { LSCOND } & 4.41 & 0.25 & 4.88 & 3.96 & 4\end{array}$


VARIABLE

MEAN

STD ERROR MAXIMUM MINIMUM

OF MEAN VALUE VALUE N

NO RAINFALL FY-1993

TSS

LTSS

TURB

LTURB

PTSASH

SCOND

LSCOND
7.72

2.15

4.35

1.56

60.30

18.78

2.98
0.83

0.09

1.18

0.31

2.59

0.28

0.01
UTR CK BELOW-

10.12

2.41

6.20

1.97

67.70

19.50

3.02
$6.40 \quad 4$ 2.004 0.904 $0.64 \quad 4$ $55.80 \quad 4$ $18.20 \quad 4$ 2.954 
VARIABLE

MEAN

STD ERROR

OF MEAN
MAXIMUM MINIMUM

VALUE

VALUE

$\mathbf{N}$ LOW RAINFALL FY-1983 UTR CK ABOVE

$\begin{array}{llllrr}\text { TSS } & 4.85 & 0.41 & 6.97 & 2.86 & 10 \\ \text { LTSS } & 1.74 & 0.07 & 2.08 & 1.35 & 10 \\ \text { TURB } & -- & -- & -- & -- & 0 \\ \text { LTURB } & -- & - & -- & -. & 0 \\ \text { PTSASH } & 47.73 & 5.67 & 72.64 & 15.38 & 8 \\ \text { SCOND } & 23.84 & 3.06 & 34.49 & 14.19 & 8 \\ \text { LSCOND } & 3.16 & 0.13 & 3.57 & 2.72 & 8\end{array}$

LOW RAINFALL FY-1983 TINKER CK

$\begin{array}{lcl}\text { TSS } & 5.20 & 0.63 \\ \text { LTSS } & 1.78 & 0.11 \\ \text { TURB } & -- & -- \\ \text { LTURB } & -- & - \\ \text { PTSASH } & 42.14 & 3.16 \\ \text { SCOND } & 38.99 & 4.98 \\ \text { LSCOND } & 3.64 & 0.14\end{array}$

LOW RAINFALL FY-1983

$\begin{array}{lcl}\text { TSS } & 6.93 & 0.75 \\ \text { LTSS } & 2.04 & 0.10 \\ \text { TURB } & -- & -- \\ \text { LTURB } & -- & - \\ \text { PTSASH } & 61.00 & 3.19 \\ \text { SCOND } & 50.07 & 5.96 \\ \text { LSCOND } & 3.91 & 0.11\end{array}$

MCQUEEN BR

7.41

2.13

$-$

54.67

57.31

4.07
$2.45 \quad 8$

1.248

$--\quad 0$

$-0$

$30.42 \quad 7$

$19.61 \quad 7$

3.037

LOW RAINFALL FY-1983 UTR CK MID.

\begin{tabular}{lr} 
TSS & 6.16 \\
LTSS & 1.94 \\
TURB & -- \\
LTURB & - \\
PTSASH & 43.81 \\
SCOND & 33.02 \\
LSCOND & 3.51 \\
& \\
\hline TSS & \\
LTSS & -- \\
TURB & -- \\
LTURB & -- \\
PTSASH & -- \\
SCOND & -- \\
LSCOND & -- \\
& -
\end{tabular}

6.16

0.76

0.11

1.94

$\overline{3} .81$

33.02

3.51
$-$

3.16

.60

0.10
8.97

2.30

--

69.10

70.36

4.27
$3.75 \quad 7$

1.567

$-\infty \quad 0$

$-$.

$54.12 \quad 5$

$36.47 \quad 5$

3.625

OW RAINFALL FY-1983 CROUCH BR 
VARIABLE

STD ERROR

MEAN

OF MEAN

MAXIMUM

MINIMUM

VALUE

VALUE

$\mathbf{N}$

LOW RAINFALL FY-1983 UTR BR BELOW

$\begin{array}{llllrl}\text { TSS } & 6.15 & 0.73 & 8.02 & 4.18 & 5 \\ \text { LTSS } & 1.94 & 0.11 & -2.20 & 1.65 & 5 \\ \text { TURB } & -- & -- & - & - & 0 \\ \text { LTURB } & -- & -- & -- & 0 \\ \text { PTSASH } & 44.41 & 1.73 & 49.35 & 41.34 & 4 \\ \text { SCOND } & 32.64 & 4.63 & 44.71 & 25.31 & 4 \\ \text { LSCOND } & 3.49 & 0.13 & 3.82 & 3.27 & 4\end{array}$

LOW RAINFALL FY-1984 UTR CK ABOVE

$\begin{array}{lrrrrr}\text { TSS } & 6.30 & 0.37 & 6.66 & 5.93 & 2 \\ \text { LTSS } & 1.99 & 0.05 & 2.04 & 1.94 & 2 \\ \text { TURB } & 3.45 & 0.85 & 4.30 & 2.60 & 2 \\ \text { LTURB } & 1.47 & 0.19 & 1.67 & 1.28 & 2 \\ \text { PTSASH } & 43.92 & 0.07 & 43.99 & 43.84 & 2 \\ \text { SCOND } & 12.88 & 0.25 & 13.13 & 12.62 & 2 \\ \text { LSCOND } & 2.63 & 0.02 & 2.65 & 2.61 & 2\end{array}$

LOW RAINFALL FY-1984 TINKER CK

$\begin{array}{lrrrrr}\text { TSS } & 9.47 & 0.73 & 10.19 & 8.74 & 2 \\ \text { LTSS } & 2.35 & 0.07 & 2.42 & 2.28 & 2 \\ \text { TURB } & 5.10 & 0.50 & 5.60 & 4.60 & 2 \\ \text { LTURB } & 1.80 & 0.08 & 1.89 & 1.72 & 2 \\ \text { PTSASH } & 47.48 & 2.86 & 50.34 & 44.62 & 2 \\ \text { SCOND } & 17.59 & 4.96 & 22.54 & 12.62 & 2 \\ \text { LSCOND } & 2.89 & 0.27 & 3.16 & 2.61 & 2\end{array}$

LOW RAINFALL FY-1984 MCQUEEN BR

$\begin{array}{lrr}\text { TSS } & 16.91 & 0.27 \\ \text { LTSS } & 2.89 & 0.02 \\ \text { TURB } & 19.00 & 1.00 \\ \text { LTURB } & 2.99 & 0.05 \\ \text { PTSASH } & 69.40 & 0.89 \\ \text { SCOND } & 22.68 & 4.43 \\ \text { LSCOND } & 3.15 & 0.19\end{array}$

$\begin{array}{rrr}17.18 & 16.64 & 2 \\ 2.90 & 2.87 & 2 \\ 20.00 & 18.00 & 2 \\ 3.04 & 2.94 & 2 \\ 70.28 & 68.51 & 2 \\ 27.12 & 18.25 & 2 \\ 3.34 & 2.96 & 2\end{array}$

LOW RAINFALL FY-1984 UTR CK MID.

$\begin{array}{lr}\text { TSS } & 10.51 \\ \text { LTSS } & 2.44 \\ \text { TURB } & 5.20 \\ \text { LTURB } & 1.82 \\ \text { PTSASH } & 52.33 \\ \text { SCOND } & 6.51 \\ \text { LSCOND } & 2.02\end{array}$

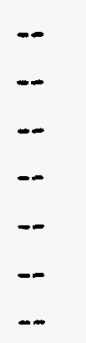

$\begin{array}{rrr}10.51 & 10.51 & 1 \\ 2.44 & 2.44 & 1 \\ 5.20 & 5.20 & 1 \\ 1.82 & 1.82 & 1 \\ 52.33 & 52.33 & 1 \\ 6.51 & 6.51 & 1 \\ 2.02 & 2.02 & 1\end{array}$


VARIABLE

MEAN

STD ERROR

MAXIMUM

OF MEAN

VALUE

MINIMUM

VALUE

$\mathbf{N}$

LOW RAINFALL FY-1984 CROUCH BR

TSS

LTSS

TURB

LTU RB

PTSASH

SCOND

LSCOND

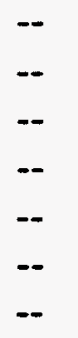

LOW RAINFALL FY-1984

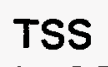

LTSS

TURB

LTURB

PTSASH

SCOND

LSCOND
9.64

2.36

5.80

1.92

52.28

5.40

1.86

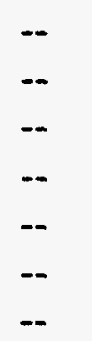

$-$

$-$

$-$

$-$

$-$
LOW RAINFALL FY-1985

TSS

LTSS

TURB

LTURB

PTSASH

SCOND

LSCOND
4.73

1.72

3.80

1.56

65.47

17.86

2.94
0.92

0.18

0.42

0.09

8.29

0.49

0.03

LOW RAINFALL FY-1985

TSS

LTSS

TURB

LTURB

PTSASH

SCOND

LSCOND
6.90

2.00

4.43

1.69

61.40

32.93

3.52
1.51

0.23

0.32

0.06

9.23

2.08

0.06

UTR CK ABOVE

9.64

2.36

5.80

1.92

52.28

5.40

1.86
9.641

$2.36 \quad 1$

$5.80 \quad 1$

1.921

$52.28 \quad 1$

$5.40 \quad 1$

$1.86 \quad 1$

LOW RAINFALL FY-1985

TSS

LTSS

TURB

LTURB

PTSASH

SCOND

LSCOND
34.75

3.42

33.60

3.48

75.65

68.55

4.17
18.75

0.58

11.60

0.35

3.75

25.14

0.38
5.70

1.90

4.40

1.69

75.90

19.15

3.00
$2.90 \quad 3$

1.363

2.993

1.383

$49.10 \quad 3$

$17.10 \quad 4$

$2.90 \quad 4$

TINKER CK

10.10

2.41

5.30

1.84

86.20

36.84

3.63

MCQUEEN BR

53.50

4.00

45.20

3.83

79.40

93.69

4.55
$2.90 \quad 4$

1.364

3.904

1.594

$44.60 \quad 4$

$27.82 \quad 4$

3.364 
VARIABLE

STD ERROR

MEAN

OF MEAN

MAXIMUM

VALUE

MINIMUM

VALUE N

LOW RAINFALL FY-1985 UTR CK MID.

$\begin{array}{lrr}\text { TSS } & 7.05 & 2.15 \\ \text { LTSS } & 2.05 & 0.27 \\ \text { TURB } & 4.70 & 0.70 \\ \text { LTURB } & 1.73 & 0.12 \\ \text { PTSASH } & 64.35 & 13.25 \\ \text { SCOND } & 20.46 & 0.73 \\ \text { LSCOND } & 3.07 & 0.03\end{array}$

LOW RAINFALL FY-1985 CROUCH BR

$\begin{array}{lr}\text { TSS } & 65.30 \\ \text { LTSS } & 4.19 \\ \text { TURB } & 158.00 \\ \text { LTURB } & 5.07 \\ \text { PTSASH } & 86.70 \\ \text { SCOND } & 78.71 \\ \text { LSCOND } & 4.38\end{array}$

65.30

4.19

2.32

5.40

1.86

77.60

21.19

3.10

$4.90 \quad 2$

1.772

4.002

1.612

$51.10 \quad 2$

19.732

3.032

--
--
--
--
--
--
--

1985
2.25
0.31
1.25
0.19
1.65
1.05
0.04

TSS

LOW RAINFALL FY-1985

$\begin{array}{lrr}\text { TSS } & 6.55 & 2.25 \\ \text { LTSS } & 1.96 & 0.31 \\ \text { TURB } & 5.75 & 1.25 \\ \text { LTURB } & 1.89 & 0.19 \\ \text { PTSASH } & 62.75 & 11.65 \\ \text { SCOND } & 24.74 & 1.05 \\ \text { LSCOND } & 3.25 & 0.04\end{array}$

LOW RAINFALL FY-1986

TSS

10.10

2.41

0.30

LTSS

5.90

1.92

LTURB

57.00

15.75

2.82

0.03

0.90

0.13

2.20

SCOND

0.15

LSCOND

0.01

158.00

5.07

86.70

78.71

4.38

65.301

4.191

$158.00 \quad 1$

5.071

$86.70 \quad 1$

$78.71 \quad 1$

$4.38 \quad 1$

UTR BR BELOW-

$\begin{array}{rrr}8.80 & 4.30 & 2 \\ 2.28 & 1.67 & 2 \\ 7.00 & 4.50 & 2 \\ 2.08 & 1.70 & 2 \\ 74.40 & 51.10 & 2 \\ 25.79 & 23.61 & 2 \\ 3.29 & 3.21 & 2\end{array}$

UTR CK ABOVE

$\begin{array}{rrr}10.40 & 9.80 & 2 \\ 2.43 & 2.38 & 2 \\ 6.80 & 5.00 & 2 \\ 2.05 & 1.79 & 2 \\ 59.20 & 54.80 & 2 \\ 15.90 & 15.60 & 2 \\ 2.83 & 2.81 & 2\end{array}$

LOW RAINFALL FY-1986 TINKER CK

$\begin{array}{lrr}\text { TSS } & 12.50 & 3.10 \\ \text { LTSS } & 2.58 & 0.23 \\ \text { TURB } & 4.75 & 0.15 \\ \text { LTURB } & 1.75 & 0.03 \\ \text { PTSASH } & 50.80 & 1.80 \\ \text { SCOND } & 30.40 & 1.60 \\ \text { LSCOND } & 3.45 & 0.05\end{array}$

15.60

9.402

2.81

2.342

4.90

4.602

1.77

1.722

52.60

$49.00 \quad 2$

32.00

$28.80 \quad 2$

3.50

3.392 


\begin{tabular}{lccccc} 
VARIABLE & MEAN & $\begin{array}{c}\text { STD ERROR } \\
\text { OF MEAN }\end{array}$ & $\begin{array}{c}\text { MAXIMUM } \\
\text { VALUE }\end{array}$ & $\begin{array}{c}\text { MINIMUM } \\
\text { VALUE }\end{array}$ & N \\
& & LOW RAINFALL & FY-1986 & MCOUEEN BR & \\
\hline TSS & 46.40 & -- & 46.40 & 46.40 & 1 \\
LTSS & 3.86 & -- & 3.86 & 3.86 & 1 \\
TURB & 79.00 & -- & 79.00 & 79.00 & 1 \\
LTURB & 4.38 & -- & 4.38 & 4.38 & 1 \\
PTSASH & 88.40 & -- & 38.40 & 88.40 & 1 \\
SCOND & 66.60 & -- & 66.60 & 66.60 & 1 \\
LSCOND & 4.21 & -- & 4.21 & 4.21 & 1
\end{tabular}

LOW RAINFALL FY-1986 UTR CK MID.

$\begin{array}{lrr}\text { TSS } & 13.90 & - \\ \text { LTSS } & 2.70 & - \\ \text { TURB } & 5.10 & - \\ \text { LTURB } & 1.81 & - \\ \text { PTSASH } & 54.70 & - \\ \text { SCOND } & 22.10 & - \\ \text { LSCOND } & 3.14 & -\end{array}$

--
--
--
--
--
-

13.90
2.70
5.10
1.81
54.70
22.10
3.14

13.90

2.70

5.10

1.81

54.70

22.10

3.14

LOW RAINFALL FY-1986 CROUCH BR

$\begin{array}{lrrr}\text { TSS } & 62.50 & - & 62.50 \\ \text { LTSS } & 4.15 & -- & 4.15 \\ \text { TURB } & 110.00 & -- & 110.00 \\ \text { LTURB } & 4.71 & -- & 4.71 \\ \text { PTSASH } & 86.20 & -- & 86.20 \\ \text { SCOND } & 104.60 & -- & 104.60 \\ \text { LSCOND } & 4.66 & -- & 4.66\end{array}$

62.50

4.15

110.00

4.71

86.20

104.60

4.66

LOW RAINFALL FY-1986 UTR CK BELOW

\begin{tabular}{lr}
\hline TSS & 14.80 \\
LTSS & 2.76 \\
TURB & 5.70 \\
LTURB & 1.90 \\
PTSASH & 54.10 \\
SCOND & 22.50 \\
LSCOND & 3.16
\end{tabular}

$$
\begin{array}{r}
14.80 \\
2.76 \\
5.70 \\
1.90 \\
54.10 \\
22.50 \\
3.16
\end{array}
$$

$\begin{array}{rr}14.80 & 1 \\ 2.76 & 1 \\ 5.70 & 1 \\ 1.90 & 1 \\ 54.10 & 1 \\ 22.50 & 1 \\ 3.16 & 1\end{array}$

LOW RAINFALL FY-1987 UTR CK ABOVE

$\begin{array}{lrrrrr}\text { TSS } & 5.95 & 0.75 & 8.80 & 3.10 & 8 \\ \text { LTSS } & 1.89 & 0.12 & 2.28 & 1.41 & 8 \\ \text { TURB } & 2.36 & 0.25 & 3.40 & 1.20 & 8 \\ \text { LTURB } & 1.19 & 0.08 & 1.48 & 0.79 & 8 \\ \text { PTSASH } & 53.51 & 1.63 & 59.60 & 45.20 & 8 \\ \text { SCOND } & 15.49 & 0.39 & 17.20 & 13.90 & 8 \\ \text { LSCOND } & 2.80 & 0.02 & 2.90 & 2.70 & 8\end{array}$


VARIABLE MEAN

LOW RAINFALL

$\begin{array}{lrl}\text { TSS } & 8.13 & 1.52 \\ \text { LTSS } & 2.09 & 0.20 \\ \text { TURB } & 2.74 & 0.28 \\ \text { LTURB } & 1.30 & 0.08 \\ \text { PTSASH } & 50.23 & 0.96 \\ \text { SCOND } & 8.38 & 1.29 \\ \text { LSCOND } & 3.37 & 0.04\end{array}$

LOW RAINFALL FY- 1987

$\begin{array}{lrr}\text { TSS } & 28.67 & 12.24 \\ \text { LTSS } & 2.95 & 0.37 \\ \text { TURB } & 45.81 & 22.71 \\ \text { LTURB } & 3.06 & 0.52 \\ \text { PTSASH } & 84.46 & 3.15 \\ \text { SCOND } & 64.83 & 7.92 \\ \text { LSCOND } & 4.14 & 0.13\end{array}$

LOW RAINFALL FY-1987 UTR CK MID.

$\begin{array}{lrr}\text { TSS } & 8.13 & 1.77 \\ \text { LTSS } & 2.15 & 0.21 \\ \text { TURB } & 3.33 & 0.66 \\ \text { LTURB } & 1.43 & 0.15 \\ \text { PTSASH } & 55.65 & 1.33 \\ \text { SCOND } & 22.35 & 1.08 \\ \text { LSCOND } & 3.15 & 0.05\end{array}$

LOW RAINFALL FY-1987

\section{TSS}

LTSS

TURB

LTURB

PTSASH

SCOND

LSCOND
43.23

3.55

81.00

4.14

87.33

89.20

4.48
15.74

0.42

31.75

0.44

2.24

9.94

0.12
MAXIMUM

FY-1987 TINKER CK

MCQUEEN BR

CROUCH BR

MINIMUM

VALUE

$\mathbf{N}$

$\begin{array}{rrr}15.00 & 2.10 & 8 \\ 2.77 & 1.13 & 8 \\ 3.90 & 1.50 & 8 \\ 1.59 & 0.92 & 8 \\ 54.70 & 46.90 & 8 \\ 35.70 & 24.40 & 8 \\ 3.60 & 3.23 & 8\end{array}$

$\begin{array}{rrr}95.10 & 6.80 & 7 \\ 4.57 & 2.05 & 7 \\ 165.00 & 4.40 & 7 \\ 5.11 & 1.69 & 7 \\ 97.20 & 75.30 & 7 \\ 93.10 & 39.80 & 7 \\ 4.54 & 3.71 & 7\end{array}$

LOW RAINFALL FY-1987

$\begin{array}{lrr}\text { TSS } & 8.45 & 1.64 \\ \text { LTSS } & 2.19 & 0.21 \\ \text { TURB } & 3.55 & 0.81 \\ \text { LTURB } & 1.47 & 0.16 \\ \text { PTSASH } & 58.65 & 0.57 \\ \text { SCOND } & 22.30 & 1.05 \\ \text { LSCOND } & 3.15 & 0.04\end{array}$

$\begin{array}{rr}3.90 & 4 \\ 1.59 & 4 \\ 2.10 & 4 \\ 1.13 & 4 \\ 51.70 & 4 \\ 20.30 & 4 \\ 3.06 & 4\end{array}$

$\begin{array}{rrr}80.70 & 12.10 & 4 \\ 4.40 & 2.57 & 4 \\ 165.00 & 21.00 & 4 \\ 5.11 & 3.09 & 4 \\ 92.90 & 82.60 & 4 \\ 108.60 & 61.80 & 4 \\ 4.70 & 4.14 & 4\end{array}$

UTR CK BELOW

$\begin{array}{rrr}11.50 & 3.90 & 4 \\ 2.53 & 1.59 & 4 \\ 5.90 & 2.30 & 4 \\ 1.93 & 1.19 & 4 \\ 59.50 & 57.00 & 4 \\ 25.40 & 20.90 & 4 \\ 3.27 & 3.09 & 4\end{array}$


64

\begin{tabular}{lccccc} 
VARIABLE & MEAN & $\begin{array}{c}\text { STD ERROR } \\
\text { OF MEAN }\end{array}$ & $\begin{array}{c}\text { MAXIMUM } \\
\text { VALUE }\end{array}$ & $\begin{array}{c}\text { MINIMUM } \\
\text { VALUE }\end{array}$ & $\mathbf{N}$ \\
\hline TSS & \multicolumn{1}{c}{ VOW RAINFALL } & FY-1988 & UTR CK ABOVE & & \\
LTSS & 4.51 & 0.40 & 6.70 & 3.30 & 8 \\
TURB & 1.69 & 0.07 & 2.04 & 1.46 & 8 \\
LTURB & 2.00 & 0.30 & 3.90 & 1.20 & 8 \\
PTSASH & 1.07 & 0.09 & 1.59 & 0.79 & 8 \\
SCOND & 55.64 & 1.57 & 64.20 & 51.20 & 8 \\
LSCOND & 15.94 & 0.60 & 19.40 & 14.40 & 8 \\
& 2.83 & 0.03 & 3.02 & 2.73 & 8
\end{tabular}

LOW RAINFALL FY-1988 TINKER CK

$\begin{array}{lrrrrr}\text { TSS } & 5.49 & 0.78 & 8.40 & 2.00 & 8 \\ \text { LTSS } & 1.81 & 0.14 & 2.24 & 1.10 & 8 \\ \text { TURB } & 2.10 & 0.19 & 2.90 & 1.40 & 8 \\ \text { LTURB } & 1.12 & 0.06 & 1.36 & 0.88 & 8 \\ \text { PTSASH } & 52.25 & 0.85 & 55.50 & 48.10 & 8 \\ \text { SCOND } & 30.48 & 1.34 & 37.40 & 26.20 & 8 \\ \text { LSCOND } & 3.44 & 0.04 & 3.65 & 3.30 & 8\end{array}$

LOW RAINFALL FY-1988 MCQUEEN BR

$\begin{array}{lrrrrr}\text { TSS } & 87.18 & 41.82 & 331.20 & 4.80 & 8 \\ \text { LTSS } & 3.63 & 0.51 & 5.81 & 1.76 & 8 \\ \text { TURB } & 128.86 & 61.45 & 495.00 & 3.20 & 8 \\ \text { LTURB } & 3.89 & 0.59 & 6.21 & 1.44 & 8 \\ \text { PTSASH } & 85.91 & 1.48 & 91.10 & 79.20 & 8 \\ \text { SCOND } & 192.93 & 58.20 & 510.00 & 50.30 & 8 \\ \text { LSCOND } & 4.95 & 0.30 & 6.24 & 3.94 & 8\end{array}$

LOW RAINFALL FY-1988 UTR CK MID.

$\begin{array}{lrrrrr}\text { TSS } & 5.38 & 0.51 & 6.70 & 4.30 & 4 \\ \text { LTSS } & 1.84 & 0.08 & 2.04 & 1.67 & 4 \\ \text { TURB } & 2.58 & 0.50 & 4.00 & 1.70 & 4 \\ \text { LTURB } & 1.25 & 0.13 & 1.61 & 0.99 & 4 \\ \text { PTSASH } & 57.05 & 2.44 & 64.20 & 53.50 & 4 \\ \text { SCOND } & 22.13 & 0.53 & 23.70 & 21.50 & 4 \\ \text { LSCOND } & 3.14 & 0.02 & 3.21 & 3.11 & 4\end{array}$

LOW RAINFALL FY-1988 CROUCH BR

$\begin{array}{lrrrrr}\text { TSS } & 96.50 & 35.53 & 167.50 & 8.40 & 4 \\ \text { LTSS } & 4.15 & 0.66 & 5.13 & 2.24 & 4 \\ \text { TURB } & 175.65 & 66.18 & 300.00 & 8.60 & 4 \\ \text { LTURB } & 4.60 & 0.80 & 5.71 & 2.26 & 4 \\ \text { PTSASH } & 88.45 & 1.73 & 90.70 & 83.30 & 4 \\ \text { SCOND } & 117.60 & 5.41 & 127.00 & 102.00 & 4 \\ \text { LSCOND } & 4.77 & 0.05 & 4.85 & 4.63 & 4\end{array}$


VARIABLE

STD ERROR

OF MEAN
MAXIMUM
VALUE
MINIMUM

VALUE

$\mathbf{N}$

LOW RAINFALL FY-1988 UTR CK BELOW

$\begin{array}{lrrrrr}\text { TSS } & 5.55 & 0.54 & 6.70 & 4.10 & 4 \\ \text { LTSS } & 1.87 & 0.09 & 2.04 & 1.63 & 4 \\ \text { TURB } & 2.58 & 0.45 & 3.70 & 1.80 & 4 \\ \text { LTURB } & 1.25 & 0.12 & 1.55 & 1.03 & 4 \\ \text { PTSASH } & 59.10 & 1.56 & 62.70 & 56.10 & 4 \\ \text { SCOND } & 22.93 & 0.43 & 24.20 & 22.30 & 4 \\ \text { LSCOND } & 3.17 & 0.02 & 3.23 & 3.15 & 4\end{array}$

LOW RAINFALL FY-1989 UTR CK ABOVE

$\begin{array}{lrlrrr} & & & & \\ \text { TSS } & 3.60 & 0.38 & 5.90 & 2.30 & 8 \\ \text { LTSS } & 1.50 & 0.08 & 1.93 & 1.19 & 8 \\ \text { TURB } & 0.59 & 0.12 & 1.40 & 0.36 & 8 \\ \text { LTURB } & 0.45 & 0.06 & 0.88 & 0.31 & 8 \\ \text { PTSASH } & 52.21 & 0.92 & 56.90 & 48.50 & 8 \\ \text { SCOND } & 16.15 & 0.29 & 17.20 & 15.10 & 8 \\ \text { LSCOND } & 2.84 & 0.02 & 2.90 & 2.78 & 8\end{array}$

LOW RAINFALL FY-1989 TINKER CK

\begin{tabular}{lrrrrr}
\hline TSS & 8.23 & 3.51 & 32.50 & 3.20 & 8 \\
LTSS & 1.94 & 0.24 & 3.51 & 1.44 & 8 \\
TURB & 5.41 & 4.38 & 36.00 & 0.43 & 8 \\
LTURB & 1.01 & 0.39 & 3.61 & 0.36 & 8 \\
PTSASH & 52.94 & 2.03 & 61.50 & 46.30 & 8 \\
SCOND & 33.33 & 1.15 & 37.50 & 28.90 & 8 \\
LSCOND & 3.53 & 0.03 & 3.65 & 3.40 & 8
\end{tabular}

LOW RAINFALL FY-1989 MCQUEEN BR

$\begin{array}{lrr}\text { TSS } & 23.78 & 7.01 \\ \text { LTSS } & 2.91 & 0.31 \\ \text { TURB } & 40.80 & 12.11 \\ \text { LTURB } & 3.35 & 0.37 \\ \text { PTSASH } & 85.01 & 1.42 \\ \text { SCOND } & 60.84 & 2.36 \\ \text { LSCOND } & 4.12 & 0.04\end{array}$

64.10
4.18
110.00
4.71
88.50
75.70
4.34

4.10
4.18

10.00

4.71

5.70

4.34

$\begin{array}{rr}4.00 & 8 \\ 1.61 & 8 \\ 4.00 & 8 \\ 1.61 & 8 \\ 76.10 & 8 \\ 53.50 & 8 \\ 4.00 & 8\end{array}$

LOW RAINFALL FY-1989 UTR CK MID.

$\begin{array}{lrr}\text { TSS } & 4.55 & 0.81 \\ \text { LTSS } & 1.68 & 0.14 \\ \text { TURB } & 1.12 & 0.56 \\ \text { LTURB } & 0.66 & 0.23 \\ \text { PTSASH } & 52.88 & 2.52 \\ \text { SCOND } & 23.48 & 0.45 \\ \text { LSCOND } & 3.20 & 0.02\end{array}$

6.90

2.07

2.80

1.34

59.60

24.60

3.24

3.20

1.44

0.41

0.34

47.60

22.70

3.17

4
4
4
4
4
4
4


66

\begin{tabular}{lrr} 
VARIABLE & MEAN & $\begin{array}{r}\text { STD ERROR } \\
\text { OF MEAN }\end{array}$ \\
& & \multicolumn{2}{c}{ LOW RAINFALL } & FY \\
\hdashline TSS & 79.73 & 39.19 \\
LTSS & 3.95 & 0.57 \\
TURB & 148.75 & 70.35 \\
LTURB & 4.58 & 0.56 \\
PTSASH & 86.48 & 2.53 \\
SCOND & 116.75 & 7.51 \\
LSCOND & 4.76 & 0.06
\end{tabular}

MAXIMUM MINIMUM
VALUE

$\mathbf{N}$

LOW RAINFALL FY-1989

$\begin{array}{lrl}\text { TSS } & 4.93 & 0.68 \\ \text { LTSS } & 1.76 & 0.12 \\ \text { TURB } & 1.39 & 0.77 \\ \text { LTURB } & 0.74 & 0.27 \\ \text { PTSASH } & 57.03 & 1.66 \\ \text { SCOND } & 24.18 & 0.45 \\ \text { LSCOND } & 3.23 & 0.02\end{array}$

LOW RAINFALL FY-1990

$\begin{array}{lrr}\text { TSS } & 6.48 & 0.92 \\ \text { LTSS } & 1.97 & 0.13 \\ \text { TURB } & 1.20 & 0.30 \\ \text { LTURB } & 0.74 & 0.14 \\ \text { PTSASH } & 54.88 & 0.91 \\ \text { SCOND } & 15.48 & 0.19 \\ \text { LSCOND } & 2.80 & 0.01\end{array}$

LOW RAINFALL FY-1990

$\begin{array}{lrl}\text { TSS } & 12.55 & 3.07 \\ \text { LTSS } & 2.47 & 0.24 \\ \text { TURB } & 2.54 & 0.63 \\ \text { LTURB } & 1.17 & 0.24 \\ \text { PTSASH } & 52.88 & 1.35 \\ \text { SCOND } & 34.47 & 1.58 \\ \text { LSCOND } & 3.56 & 0.04\end{array}$

LOW RAINFALL FY-1990 MCQUEEN BR

$\begin{array}{lrl}\text { TSS } & 5.97 & 1.54 \\ \text { LTSS } & 1.84 & 0.19 \\ \text { TURB } & 3.64 & 1.02 \\ \text { LTURB } & 1.41 & 0.22 \\ \text { PTSASH } & 74.80 & 2.65 \\ \text { SCOND } & 58.18 & 3.25 \\ \text { LSCOND } & 4.07 & 0.06\end{array}$

13.10

2.64

7.70

2.16

83.70

67.30

4.22
3.30
1.46
0.51
0.41
54.60
23.30
3.19

16.60

2.87

31.00

3.47

80.80

103.00

4.64

UTR CK BELOW

$\begin{array}{rrr}6.60 & 3.30 & 4 \\ 2.03 & 1.46 & 4 \\ 3.70 & 0.51 & 4 \\ 1.55 & 0.41 & 4 \\ 61.90 & 54.60 & 4 \\ 25.00 & 23.30 & 4 \\ 3.26 & 3.19 & 4\end{array}$

UTR CK ABOVE

9.20
2.32
2.20
1.16
57.00
16.10
2.84

3.50

1.50

0.41

0.34

4

4

4

4

4

4

4

TINKER CK

$\begin{array}{rrr}25.20 & 4.00 & 6 \\ 3.26 & 1.61 & 6 \\ 4.30 & 0.73 & 6 \\ 1.67 & 0.55 & 6 \\ 56.50 & 48.10 & 6 \\ 39.90 & 29.50 & 6 \\ 3.71 & 3.42 & 6\end{array}$

$51.30 \quad 6$

$14.80 \quad 6$

$2.76 \quad 6$

6
6
6
6
6
6
6


VARIABLE

MEAN

STD ERROR

OF MEAN

MAXIMUM

MINIMUM

VALUE

VALUE

$\mathbf{N}$

LOW RAINFALL FY-1990 UTR CK MID.

$\begin{array}{lrl} & & \\ \text { TSS } & 7.73 & 1.54 \\ \text { LTSS } & 2.13 & 0.20 \\ \text { TURB } & 1.31 & 0.65 \\ \text { LTURB } & 0.76 & 0.27 \\ \text { PTSASH } & 52.03 & 3.27 \\ \text { SCOND } & 20.53 & 0.26 \\ \text { LSCOND } & 3.07 & 0.01\end{array}$

9.70

2.37

4.70

3

2.60

1.74

0.47

3

1.28

0.39

3

55.30

45.50

21.00

20.10

3.09

3.05

3

3

3

3

LOW RAINFALL FY- 1990 CROUCH BR

$\begin{array}{lrr}\text { TSS } & 15.57 & 2.11 \\ \text { LTSS } & 2.79 & 0.13 \\ \text { TURB } & 21.57 & 6.54 \\ \text { LTURB } & 3.00 & 0.37 \\ \text { PTSASH } & 69.30 & 2.95 \\ \text { SCOND } & 74.70 & 14.00 \\ \text { LSCOND } & 4.29 & 0.18\end{array}$

LOW RAINFALL FY-1990

$\begin{array}{lrl}\text { TSS } & 7.67 & 1.85 \\ \text { LTSS } & 2.10 & 0.25 \\ \text { TURB } & 1.68 & 0.57 \\ \text { LTURB } & 0.93 & 0.25 \\ \text { PTSASH } & 55.33 & 1.19 \\ \text { SCOND } & 21.87 & 0.50 \\ \text { LSCOND } & 3.13 & 0.02\end{array}$

UTR CK BELOW

9.90
2.39
2.40
1.22
57.30
22.60
3.16

18.80

2.99

30.00

3.43

75.00

102.00

4.63

11.60

2.53

8.70

2.27

65.10

55.70

4.04

3

3

3

3

3

3

3

3

LOW RAINFALL FY-1991 UTR CK ABOVE

\begin{tabular}{lcc} 
& \multicolumn{1}{c}{ LOW RAINFALL } & FY- \\
\hline TSS & 7.78 & 1.39 \\
LTSS & 2.04 & 0.14 \\
TURB & 1.63 & 0.41 \\
LTURB & 0.85 & 0.12 \\
PTSASH & 53.08 & 1.37 \\
SCOND & 16.19 & 0.77 \\
LSCOND & 2.83 & 0.04
\end{tabular}

19.03

3.00

5.60

1.89

62.70

25.80

3.29

4.00

1.61

0.55

0.44

53.20

20.90

3.09

LOW RAINFALL FY-1991 TINKER CK

\begin{tabular}{lrr} 
& LOW RAINFALL & FY \\
\hline TSS & 7.79 & 1.31 \\
LTSS & 2.02 & 0.15 \\
TURB & 1.23 & 0.25 \\
LTURB & 0.73 & 0.10 \\
PTSASH & 48.16 & 1.85 \\
SCOND & 27.41 & 0.98 \\
LSCOND & 3.34 & 0.03
\end{tabular}

17.75

2.93

3.50

1.50

55.60

32.80

3.52

2.91

1.36

0.35

0.30

45.50

14.90

2.77
14

14

14

14

14

14

14 


\begin{tabular}{lccccc} 
VARIABLE & MEAN & $\begin{array}{c}\text { STD ERROR } \\
\text { OF MEAN }\end{array}$ & $\begin{array}{c}\text { MAXIMUM } \\
\text { VALUE }\end{array}$ & $\begin{array}{c}\text { MINIMUM } \\
\text { VALUE }\end{array}$ & N \\
\hline TSS & 16.80 & 4.10 & & & \\
LTSS & 2.62 & 0.19 & 51.24 & 4.86 & 14 \\
TURB & 6.86 & 3.28 & 49.9 & 1.77 & 14 \\
LTURB & 1.64 & 0.20 & 3.91 & 1.60 & 14 \\
PTSASH & 71.97 & 2.13 & 91.70 & 0.96 & 14 \\
SCOND & 42.28 & 2.12 & 61.60 & 30.00 & 14 \\
LSCOND & 3.75 & 0.05 & 4.14 & 3.44 & 14
\end{tabular}

LOW RAINFALL FY-1991 UTR CK MID.

$\begin{array}{lrl}\text { TSS } & 6.91 & 0.98 \\ \text { LTSS } & 2.01 & 0.13 \\ \text { TURB } & 1.22 & 0.20 \\ \text { LTURB } & 0.77 & 0.10 \\ \text { PTSASH } & 51.56 & 1.56 \\ \text { SCOND } & 20.34 & 0.22 \\ \text { LSCOND } & 3.06 & 0.01\end{array}$

LOW RAINFALL FY-1991 CROUCH BR

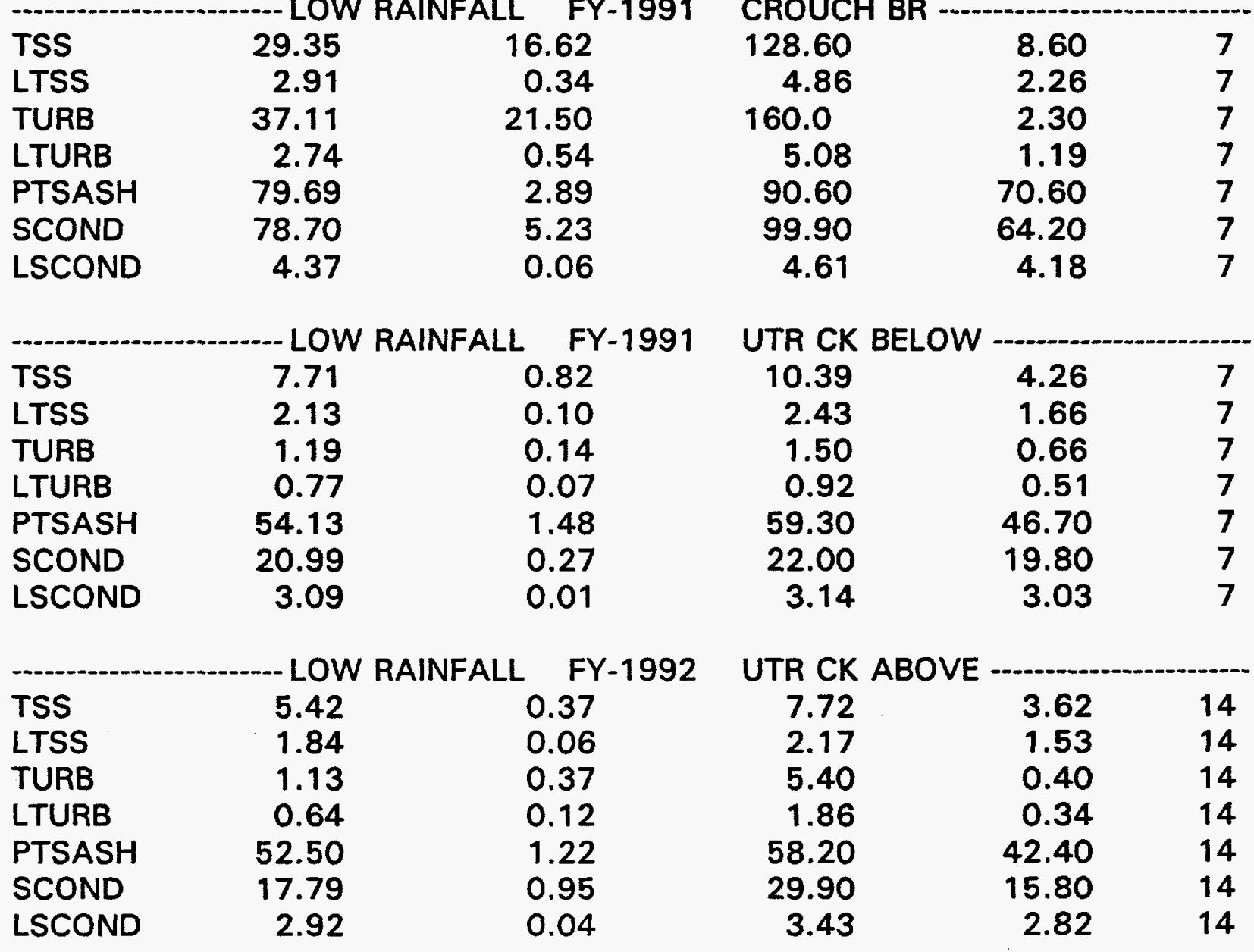


VARIABLE

\section{STD ERROR}

MAXIMUM

OF MEAN

VALUE

MINIMUM

VALUE

$\mathbf{N}$

LOW RAINFALL FY-1992 TINKER CK

$\begin{array}{lrl}\text { TSS } & 6.63 & 0.72 \\ \text { LTSS } & 1.97 & 0.10 \\ \text { TURB } & 1.42 & 0.35 \\ \text { LTURB } & 0.78 & 0.12 \\ \text { PTSASH } & 52.02 & 1.50 \\ \text { SCOND } & 26.20 & 0.91 \\ \text { LSCOND } & 3.30 & 0.04\end{array}$

LOW RAINFALL FY-1992

10.40
2.43
4.50
1.70
66.70
30.40
3.45

2.73

1.32

0.37

0.31

42.60

18.60

2.98

14

14

14

14

14

14

14

TSS
LTSS
TURB
LTURB
PTSASH
SCOND
LSCOND

18.20

5.47

2.54

23.27

0.24

2.16

9.78

75.88

0.38

1.94

45.40

3.82

2.22

0.04

LOW RAINFALL FY-1992 UTR CK MID.

$\begin{array}{lrr}\text { TSS } & 7.82 & 2.01 \\ \text { LTSS } & 2.06 & 0.19 \\ \text { TURB } & 2.50 & 1.56 \\ \text { LTURB } & 0.90 & 0.29 \\ \text { PTSASH } & 55.64 & 1.91 \\ \text { SCOND } & 21.86 & 0.48 \\ \text { LSCOND } & 3.13 & 0.02\end{array}$

LOW RAINFALL FY-1992 CROUCH BR

$\begin{array}{lrr}\text { TSS } & 34.26 & 11.09 \\ \text { LTSS } & 3.23 & 0.35 \\ \text { TURB } & 52.04 & 19.92 \\ \text { LTURB } & 3.28 & 0.55 \\ \text { PTSASH } & 79.71 & 4.03 \\ \text { SCOND } & 82.71 & 3.43 \\ \text { LSCOND } & 4.42 & 0.04\end{array}$

LOW RAINFALL FY-1992

\begin{tabular}{|c|c|c|c|c|c|}
\hline & - LOW & FY-1992 & UTR CK & & \\
\hline TSS & 8.18 & 1.90 & 18.6 & 4.13 & 7 \\
\hline LTSS & 2.12 & 0.17 & 2.98 & 1.64 & 7 \\
\hline TURB & 2.70 & 1.65 & 12.50 & 0.49 & 7 \\
\hline LTURB & 0.97 & 0.29 & 2.60 & 0.40 & 7 \\
\hline PTSASH & 56.34 & 2.24 & 63.40 & 46.60 & 7 \\
\hline SCOND & 22.60 & 0.59 & 24.60 & 20.20 & 7 \\
\hline LSCOND & 3.16 & 0.03 & 3.24 & 3.05 & 7 \\
\hline
\end{tabular}

87.21

4.48

140.00

4.95

88.60

93.40

4.55
4.18

1.64

1.50

0.92

66.30

37.70

3.66
14

14

14

14

14

14

14 
VARIABLE MEAN

STD ERROR
OF MEAN

MAXIMUM
VALUE

MINIMUM

VALUE

$\mathbf{N}$

LOWRAINFALL FY-1993 UTR CK ABOVE

$\begin{array}{lrrrrr}\text { TSS } & 4.79 & 0.35 & 6.21 & 3.60 & 8 \\ \text { LTSS } & 1.74 & 0.06 & 1.98 & 1.53 & 8 \\ \text { TURB } & 3.45 & 0.75 & 8.50 & 2.10 & 8 \\ \text { LTURB } & 1.42 & 0.13 & 2.25 & 1.13 & 8 \\ \text { PTSASH } & 56.34 & 1.79 & 64.50 & 47.60 & 8 \\ \text { SCOND } & 17.06 & 0.60 & 19.50 & 14.80 & 8 \\ \text { LSCOND } & 2.89 & 0.03 & 3.02 & 2.76 & 8\end{array}$

LOW RAINFALL FY-1993 TINKER CK

$\begin{array}{lrrrrr}\text { TSS } & 4.38 & 0.64 & 7.66 & 2.12 & 8 \\ \text { LTSS } & 1.63 & 0.12 & 2.16 & 1.14 & 8 \\ \text { TURB } & 2.85 & 0.26 & 4.30 & 2.10 & 8 \\ \text { LTURB } & 1.33 & 0.06 & 1.67 & 1.13 & 8 \\ \text { PTSAS } & 47.93 & 2.41 & 53.40 & 32.00 & 8 \\ \text { SCOND } & 24.66 & 0.93 & 28.40 & 21.30 & 8 \\ \text { LSCOND } & 3.24 & 0.04 & 3.38 & 3.10 & 8\end{array}$

LOW RAINFALL FY-1993 MCQUEEN BR

$\begin{array}{lrr}\text { TSS } & 6.87 & 1.28 \\ \text { LTSS } & 1.98 & 0.16 \\ \text { TURB } & 12.93 & 2.74 \\ \text { LTURB } & 2.50 & 0.20 \\ \text { PTSASH } & 78.39 & 1.68 \\ \text { SCOND } & 40.74 & 1.97 \\ \text { LSCOND } & 3.72 & 0.05\end{array}$

LOW RAINFALL FY-1993 UTR CK MID.

$\begin{array}{lrrrrr}\text { TSS } & 4.58 & 0.57 & 5.83 & 3.08 & 4 \\ \text { LTSS } & 1.70 & 0.11 & 1.92 & 1.41 & 4 \\ \text { TURB } & 3.55 & 0.52 & 4.80 & 2.60 & 4 \\ \text { LTURB } & 1.50 & 0.11 & 1.76 & 1.28 & 4 \\ \text { PTSASH } & 54.55 & 1.25 & 57.10 & 52.20 & 4 \\ \text { SCOND } & 21.06 & 1.10 & 23.50 & 18.20 & 4 \\ \text { LSCOND } & 3.09 & 0.05 & 3.20 & 2.95 & 4\end{array}$

LOW RAINFALL FY-1993 CROUCH BR

$\begin{array}{lrr}\text { TSS } & 29.98 & 8.41 \\ \text { LTSS } & 3.32 & 0.28 \\ \text { TURB } & 56.25 & 14.30 \\ \text { LTURB } & 3.93 & 0.30 \\ \text { PTSASH } & 85.75 & 3.44 \\ \text { SCOND } & 88.85 & 16.16 \\ \text { LSCOND } & 4.45 & 0.17\end{array}$

$\begin{array}{rrr}52.50 & 12.90 & 4 \\ 3.98 & 2.63 & 4 \\ 91.00 & 21.00 & 4 \\ 4.52 & 3.09 & 4 \\ 90.70 & 75.60 & 4 \\ 135.00 & 59.50 & 4 \\ 4.91 & 4.10 & 4\end{array}$


VARIABLE

MEAN

STD ERROR MAXIMUM MINIMUM OF MEAN VALUE VALUE $\mathbf{N}$

LOW RAINFALL FY-1993 UTR CK BELOW

$\begin{array}{lr}\text { TSS } & 4.84 \\ \text { LTSS } & 1.75 \\ \text { TURB } & 4.35 \\ \text { LTURB } & 1.63 \\ \text { PTSASH } & 56.73 \\ \text { SCOND } & 21.78 \\ \text { LSCOND } & 3.12\end{array}$

0.66

0.12

0.93

0.18

1.18

1.24

6.31

1.99

6.10

1.96

60.10

24.40

3.23

$3.38 \quad 4$

1.484

$2.70 \quad 4$

1.314

$54.60 \quad 4$

0.06 $18.50 \quad 4$ 2.974 
STD ERROR MAXIMUM MINIMUM

VARIABLE MEAN

OF MEAN VALUE VALUE N

HIGH RAINFALL FY-1983 UTR CK ABOVE

$\begin{array}{llllrr}\text { TSS } & 9.36 & 1.62 & 22.20 & 5.26 & 10 \\ \text { LTSS } & 2.25 & 0.13 & 3.14 & 1.83 & 10 \\ \text { TURB } & -- & -- & -- & -- & 0 \\ \text { LTURB } & - & - & -- & -- & 0 \\ \text { PTSASH } & 53.04 & 0.74 & 57.07 & 49.62 & 10 \\ \text { SCOND } & 29.15 & 1.62 & 37.09 & 25.41 & 7 \\ \text { LSCOND } & 3.40 & 0.05 & 3.64 & 3.27 & 7\end{array}$

HIGH RAINFALL FY-1983 TINKER CK

$\begin{array}{lccccc}\text { TSS } & 11.15 & 2.10 & 21.79 & 4.76 & 9 \\ \text { LTSS } & 2.39 & 0.16 & 3.13 & 1.75 & 9 \\ \text { TURB } & -- & -- & -- & -- & 0 \\ \text { LTURB } & -- & - & -- & -- & 0 \\ \text { PTSASH } & 52.00 & 4.96 & 90.54 & 41.53 & 9 \\ \text { SCOND } & 44.37 & 1.05 & 48.26 & 39.63 & 7 \\ \text { LSCOND } & 3.81 & 0.02 & 3.90 & 3.70 & 7\end{array}$

HIGH RAINFALL FY-1983 MCQUEEN BR

$\begin{array}{lccccc}\text { TSS } & 59.04 & 16.31 & 114.97 & 8.89 & 6 \\ \text { LTSS } & 3.81 & 0.38 & 4.75 & 2.29 & 6 \\ \text { TURB } & -- & -- & -- & -- & 0 \\ \text { LTURB } & -- & -- & -- & -- & 0 \\ \text { PTSASH } & 76.81 & 3.06 & 82.34 & 62.59 & 6 \\ \text { SCOND } & 51.28 & 6.23 & 63.57 & 43.36 & 3 \\ \text { LSCOND } & 3.94 & 0.11 & 4.17 & 3.79 & 3\end{array}$

HIGH RAINFALL FY-1983 UTR CK MID.

$\begin{array}{lccccc}\text { TSS } & 28.59 & 12.81 & 73.74 & 8.00 & 5 \\ \text { LTSS } & 3.01 & 0.43 & 4.31 & 2.20 & 5 \\ \text { TURB } & -- & -- & -- & -- & 0 \\ \text { LTURB } & -- & -- & -- & 5 & 0 \\ \text { PTSASH } & 63.25 & 4.27 & 75.96 & 5.97 & 5 \\ \text { SCOND } & 34.80 & 3.19 & 39.69 & 25.84 & 4 \\ \text { LSCOND } & 3.56 & 0.10 & 3.71 & 3.29 & 4\end{array}$

HIGH RAINFALL FY-1983 CROUCH BR

TSS
LTSS
TU RB
LTU RB
PTSASH
SCOND
LSCOND


VARIABLE MEAN

STD ERROR
OF MEAN
MAXIMUM
VALUE
MINIMUM

VALUE

$\mathbf{N}$

HIGH RAINFALL FY-1983

$\begin{array}{lcc}\text { TSS } & 39.01 & 18.60 \\ \text { LTSS } & 3.24 & 0.48 \\ \text { TURB } & -- & - \\ \text { LTURB } & -- & -- \\ \text { PTSASH } & 60.64 & 9.35 \\ \text { SCOND } & 34.19 & 2.50 \\ \text { LSCOND } & 3.55 & 0.08\end{array}$

UTR CK BELOW

$\begin{array}{ccc}105.70 & 8.12 & 5 \\ 4.67 & 2.21 & 5 \\ -- & -- & 0 \\ -- & -- & 0 \\ 78.03 & 29.90 & 5 \\ 38.17 & 27.01 & 4 \\ 3.67 & 3.33 & 4\end{array}$

HIGH RAINFALL FY- 1984 UTR CK ABOVE

$\begin{array}{lcc} & & \text { HIGH RAINFALL } \\ \text { TSS } & 8.03 & 0.32 \\ \text { LTSS } & 2.20 & 0.03 \\ \text { TURB } & 7.55 & 2.15 \\ \text { LTURB } & 2.11 & 0.26 \\ \text { PTSASH } & 57.75 & 0.29 \\ \text { SCOND } & 19.47 & 2.18 \\ \text { LSCOND } & 3.01 & 0.11\end{array}$
8.34
2.23
9.70
2.37
58.03
21.65
3.12

7.71

2.16

5.40

1.86

57.46

17.28

2.91

HIGH RAINFALL FY-1984

$\begin{array}{lrr} & 11.34 & 8.42 \\ \text { TSS } & 2.20 & 0.83 \\ \text { LTSS } & 3.60 & 0.10 \\ \text { TURB } & 1.53 & 0.02 \\ \text { LTURB } & 48.79 & 12.49 \\ \text { PTSASH } & 21.38 & 0.09 \\ \text { SCOND } & 2.11 & <0.01\end{array}$

TINKER CK

$\begin{array}{rrr}19.76 & 2.92 & 2 \\ 3.03 & 1.37 & 2 \\ 3.70 & 3.50 & 2 \\ 1.55 & 1.50 & 2 \\ 61.29 & 36.30 & 2 \\ 21.47 & 21.30 & 2 \\ 3.11 & 3.10 & 2\end{array}$

HIGH RAINFALL FY-1984

$\begin{array}{lrr}\text { TSS } & 37.35 & 2.01 \\ \text { LTSS } & 3.65 & 0.05 \\ \text { TURB } & 54.00 & 8.00 \\ \text { LTURB } & 4.00 & 0.15 \\ \text { PTSASH } & 78.00 & 0.92 \\ \text { SCOND } & 23.52 & 1.69 \\ \text { LSCOND } & 3.20 & 0.07\end{array}$

MCQUEEN BR

$\begin{array}{rrr}39.36 & 35.34 & 2 \\ 3.70 & 3.59 & 2 \\ 62.00 & 46.00 & 2 \\ 4.14 & 3.85 & 2 \\ 78.91 & 77.08 & 2 \\ 25.21 & 21.83 & 2 \\ 3.27 & 3.13 & 2\end{array}$

HIGH RAINFALL FY- 1984 UTR CK MID.

$\begin{array}{lr}\text { TSS } & 12.89 \\ \text { LTSS } & 2.63 \\ \text { TURB } & 6.40 \\ \text { LTU RB } & 2.00 \\ \text { PTSASH } & 28.16 \\ \text { SCOND } & 303.71 \\ \text { LSCOND } & 5.72\end{array}$

12.89

2.63

6.40

2.00

28.16

303.71

5.72

$\begin{array}{rr}12.89 & 1 \\ 2.63 & 1 \\ 6.40 & 1 \\ 2.00 & 1 \\ 28.16 & 1 \\ 303.71 & 1 \\ 5.72 & 1\end{array}$


VARIABLE MEAN

STD ERROR
OF MEAN
MAXIMUM
VALUE
MINIMUM

VALUE

$\mathbf{N}$

HIGH RAINFALL FY-1984 CROUCH BR

TSS
LTSS
TURB
LTURB
PTSASH
SCOND
LSCOND

HIGH RAINFALL

TSS

LTSS

TURB

LTURB

PTSASH

SCOND

LSCOND
9.82

2.38

6.90

2.07

53.87

21.60

3.12

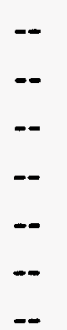

HIGH RAINFALL FY-1986

TSS

LTSS

TURB

LTURB

PTSASH

SCOND

LSCOND
14.30

2.48

4.60

1.63

52.75

18.80

2.96
6.96

0.37

1.49

0.23

1.43

2.44

0.12

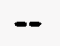

$-$

$-$

$-$

$--$

HIGH RAINFALL FY-1986

TSS

9.73

LTSS

TURB

LTURB

PTSASH

SCOND

LSCOND

2.32

4.68

1.70

51.13

44.03

3.74

1.94

0.18

0.82

0.15

1.21

9.82

0.21

HIGH RAINFALL FY-1986

$\begin{array}{lr}\text { TSS } & 32.45 \\ \text { LTSS } & 3.49 \\ \text { TURB } & 53.00 \\ \text { LTURB } & 3.95 \\ \text { PTSASH } & \mathbf{8 5 . 4 5} \\ \text { SCOND } & 75.10 \\ \text { LSCOND } & 4.31\end{array}$

6.55

0.20

14.00

0.27

3.25

14.40

0.19

FY-1984

UTR CK BELOW

$\begin{array}{rrr}9.82 & 9.82 & 1 \\ 2.38 & 2.38 & 1 \\ 6.90 & 6.90 & 1 \\ 2.07 & 2.07 & 1 \\ 53.87 & 53.87 & 1 \\ 21.60 & 21.60 & 1 \\ 3.12 & 3.12 & 1\end{array}$

UTR CK ABOVE

$\begin{array}{rrr}35.10 & 6.10 & 4 \\ 3.59 & 1.96 & 4 \\ 9.00 & 2.60 & 4 \\ 2.30 & 1.28 & 4 \\ 55.00 & 48.60 & 4 \\ 25.50 & 14.90 & 4 \\ 3.28 & 2.77 & 4\end{array}$

TINKER CK

$\begin{array}{rrr}14.90 & 5.80 & 4 \\ 2.77 & 1.92 & 4 \\ 6.50 & 3.00 & 4 \\ 2.01 & 1.39 & 4 \\ 53.40 & 47.70 & 4 \\ 71.50 & 27.40 & 4 \\ 4.28 & 3.35 & 4\end{array}$

MCQUEEN BR

$\begin{array}{rrr} & & \\ 39.00 & 25.90 & 2 \\ 3.69 & 3.29 & 2 \\ 67.00 & 39.00 & 2 \\ 4.22 & 3.69 & 2 \\ 88.70 & 82.20 & 2 \\ 89.50 & 60.70 & 2 \\ 4.51 & 4.12 & 2\end{array}$


VARIABLE MEAN

\section{STD ERROR \\ OF MEAN}
MAXIMUM
VALUE

MINIMUM

VALUE

$\mathbf{N}$

HIGH RAINFALL FY-1986

$\begin{array}{lrr} & & \\ \text { TSS } & 17.70 & 7.90 \\ \text { LTSS } & 2.83 & 0.45 \\ \text { TURB } & 11.40 & 6.60 \\ \text { LTURB } & 2.35 & 0.59 \\ \text { PTSASH } & 55.95 & 1.85 \\ \text { SCOND } & 31.70 & 10.30 \\ \text { LSCOND } & 3.44 & 0.33\end{array}$

UTR CK MID.

25.60

3.28

9.80

2.38

18.00

4.80

1.76

2.94

54.10

57.80

42.00

21.40

3.76

3.11

2
2
2
2
2
2
2

HIGH RAINFALL

$\begin{array}{lrr}\text { TSS } & 59.35 & 34.45 \\ \text { LTSS } & 3.90 & 0.65 \\ \text { TU RB } & 130.00 & 80.00 \\ \text { LTURB } & 4.64 & 0.71 \\ \text { PTSASH } & 88.75 & 0.45 \\ \text { SCOND } & 103.70 & 7.80 \\ \text { LSCOND } & 4.65 & 0.07\end{array}$

FY- 1986 CROUCH BR

$$
\begin{array}{r}
93.80 \\
4.55 \\
210.00 \\
5.35 \\
89.20 \\
111.50 \\
4.72
\end{array}
$$

$\begin{array}{rr}24.90 & 2 \\ 3.25 & 2 \\ 50.00 & 2 \\ 3.93 & 2 \\ 88.30 & 2 \\ 95.90 & 2 \\ 4.57 & 2\end{array}$

HIGH RAINFALL

$\begin{array}{lrr}\text { TSS } & 16.70 & 5.70 \\ \text { LTSS } & 2.82 & 0.33 \\ \text { TURB } & 11.55 & 7.45 \\ \text { LTURB } & 2.31 & 0.68 \\ \text { PTSASH } & 57.60 & 3.10 \\ \text { SCOND } & 30.35 & 5.95 \\ \text { LSCOND } & 3.43 & 0.19\end{array}$

FY-1986

UTR CK BELOW

22.40
3.15
19.00
2.99
60.70
36.30
3.62

11.00

2.48

2

4.10

1.63

54.50

24.40

3.23

2

2

2

2

2

HIGH RAINFALL

$\begin{array}{lrr}\text { TSS } & 7.88 & 1.33 \\ \text { LTSS } & 2.11 & 0.14 \\ \text { TURB } & 3.41 & 0.52 \\ \text { LTURB } & 1.44 & 0.11 \\ \text { PTSASH } & 51.28 & 0.66 \\ \text { SCOND } & 16.15 & 0.54 \\ \text { LSCOND } & 2.84 & 0.03\end{array}$

FY-1987 UTR CK ABOVE

15.90
2.83
6.50
2.01
54.80
18.30
2.96

4.00

8

8

1.61

8

8

8

8

8

HIGH RAINFALL

$\begin{array}{lrr}\text { TSS } & 13.71 & 3.88 \\ \text { LTSS } & 2.51 & 0.23 \\ \text { TURB } & 7.49 & 2.78 \\ \text { LTURB } & 1.94 & 0.22 \\ \text { PTSASH } & 51.99 & 1.25 \\ \text { SCOND } & 29.86 & 1.54 \\ \text { LSCOND } & 3.42 & 0.05\end{array}$

FY-1987 TINKER CK

34.20
3.56
24.00
3.22
58.20
35.60
3.60

$\begin{array}{rr}4.20 & 7 \\ 1.65 & 7 \\ 3.20 & 7 \\ 1.44 & 7 \\ 49.10 & 7 \\ 24.50 & 7 \\ 3.24 & 7\end{array}$


VARIABLE MEAN

\section{STD ERROR \\ OF MEAN}

MAXIMUM
VALUE

MINIMUM

VALUE

$\mathbf{N}$

HIGH RAINFALL FY- 1987 MCQUEEN BR

$\begin{array}{lrrrrr}\text { TSS } & 256.04 & 75.18 & 655.00 & 54.00 & 8 \\ \text { LTSS } & 5.20 & 0.33 & 6.49 & 4.01 & 8 \\ \text { TURB } & 327.44 & 75.05 & 650.00 & 88.50 & 8 \\ \text { LTURB } & 5.56 & 0.28 & 6.48 & 4.49 & 8 \\ \text { PTSASH } & 89.70 & 0.96 & 93.20 & 85.40 & 8 \\ \text { SCOND } & 75.55 & 10.09 & 118.30 & 41.70 & 8 \\ \text { LSCOND } & 4.28 & 0.13 & 4.78 & 3.75 & 8\end{array}$

HIGH RAINFALL FY-1987 UTR CK MID.

$\begin{array}{lrrrrr}\text { TSS } & 14.65 & 5.16 & 29.10 & 5.20 & 4 \\ \text { LTSS } & 2.59 & 0.33 & 3.40 & 1.82 & 4 \\ \text { TURB } & 12.83 & 5.81 & 29.00 & 2.50 & 4 \\ \text { LTURB } & 2.34 & 0.45 & 3.40 & 1.25 & 4 \\ \text { PTSASH } & 63.05 & 3.74 & 71.30 & 55.80 & 4 \\ \text { SCOND } & 22.50 & 1.26 & 25.90 & 20.10 & 4 \\ \text { LSCOND } & 3.15 & 0.05 & 3.29 & 3.05 & 4\end{array}$

HIGH RAINFALL FY-1987 CROUCH BR

$\begin{array}{lrrrrr}\text { TSS } & 119.75 & 34.79 & 215.50 & 50.40 & 4 \\ \text { LTSS } & 4.67 & 0.30 & 5.38 & 3.94 & 4 \\ \text { TURB } & 216.75 & 60.07 & 390.00 & 97.00 & 4 \\ \text { LTURB } & 5.26 & 0.28 & 5.97 & 4.58 & 4 \\ \text { PTSASH } & 88.78 & 0.86 & 90.50 & 86.50 & 4 \\ \text { SCOND } & 110.55 & 9.58 & 127.20 & 84.30 & 4 \\ \text { LSCOND } & 4.70 & 0.09 & 4.85 & 4.45 & 4\end{array}$

HIGH RAINFALL FY-1987 UTR CK BELOW

$\begin{array}{lrrrrr}\text { TSS } & 20.15 & 6.48 & 36.80 & 6.60 & 4 \\ \text { LTSS } & 2.89 & 0.34 & 3.63 & 2.03 & 4 \\ \text { TURB } & 20.78 & 7.56 & 40.00 & 3.10 & 4 \\ \text { LTURB } & 2.80 & 0.49 & 3.71 & 1.41 & 4 \\ \text { PTSASH } & 68.40 & 3.56 & 76.10 & 62.10 & 4 \\ \text { SCOND } & 23.73 & 1.26 & 26.30 & 20.60 & 4 \\ \text { LSCOND } & 3.20 & 0.05 & 3.31 & 3.07 & 4\end{array}$

HIGH RAINFALL FY-1988 UTR CK ABOVE

$\begin{array}{lrl}\text { TSS } & 13.34 & 1.97 \\ \text { LTSS } & 2.54 & 0.18 \\ \text { TURB } & 8.76 & 2.20 \\ \text { LTURB } & 2.04 & 0.24 \\ \text { PTSASH } & 58.07 & 1.08 \\ \text { SCOND } & 19.25 & 1.21 \\ \text { LSCOND } & 2.99 & 0.06\end{array}$

22.40

3.15

24.00

3.22

65.20

26.90

3.33
3.10

1.41

1.50

0.92

53.80

14.70

2.75 
VARIABLE MEAN

\section{STD ERROR OF MEAN}

MAXIMUM

MINIMUM

VALUE

VALUE

$\mathbf{N}$

HIGH RAINFALL FY-1988 TINKER CK

$\begin{array}{lrr}\text { TSS } & 24.28 & 4.44 \\ \text { LTSS } & 2.99 & 0.27 \\ \text { TURB } & 14.34 & 4.05 \\ \text { LTURB } & 2.40 & 0.29 \\ \text { PTSASH } & 57.46 & 2.55 \\ \text { SCOND } & 36.80 & 3.54 \\ \text { LSCOND } & 3.59 & 0.10\end{array}$

45.00

3.83

2.20

10

45.00

1.16

10

3.83

1.60

72.40

0.96

55.30

49.90

10

4.03

22.30

10

3.15

10

HIGH RAINFALL FY-1988 MCQUEEN

$\begin{array}{lrr}\text { TSS } & 162.56 & 30.03 \\ \text { LTSS } & 4.86 & 0.26 \\ \text { TURB } & 237.30 & 39.69 \\ \text { LTURB } & 5.27 & 0.24 \\ \text { PTSASH } & 88.39 & 0.43 \\ \text { SCOND } & 101.16 & 28.62 \\ \text { LSCOND } & 4.40 & 0.20\end{array}$

HIGH RAINFALL FY-1988 UTR CK MID.

$\begin{array}{lrr}\text { TSS } & 34.22 & 9.90 \\ \text { LTSS } & 3.38 & 0.32 \\ \text { TURB } & 40.92 & 11.91 \\ \text { LTURB } & 3.43 & 0.47 \\ \text { PTSASH } & 73.66 & 2.63 \\ \text { SCOND } & 30.18 & 3.20 \\ \text { LSCOND } & 3.42 & 0.11\end{array}$

HIGH RAINFALL FY-1988

$\begin{array}{lrr}\text { TSS } & 250.94 & 47.84 \\ \text { LTSS } & 5.45 & 0.20 \\ \text { TURB } & 430.00 & 74.10 \\ \text { LTURB } & 6.01 & 0.17 \\ \text { PTSASH } & 90.26 & 0.67 \\ \text { SCOND } & 108.32 & 10.34 \\ \text { LSCOND } & 4.67 & 0.10\end{array}$

273.90

5.62

395.00

5.98

90.10

328.00

5.80

66.90

4.22

74.00

4.32

78.90

38.40

3.67

CROUCH BR

398.30

5.99

670.00

6.51

92.60

128.50

4.86
22.00

3.14

35.00

3.58

86.40

46.70

3.86
10

10

10

10

10

10

10

HIGH RAINFALL FY-1988 UTR CK BELOW

$\begin{array}{lrr}\text { TSS } & 43.46 & 9.73 \\ \text { LTSS } & 3.63 & 0.33 \\ \text { TURB } & 50.88 & 12.37 \\ \text { LTURB } & 3.68 & 0.46 \\ \text { PTSASH } & 74.80 & 2.69 \\ \text { SCOND } & 32.50 & 3.30 \\ \text { LSCOND } & 3.49 & 0.10\end{array}$

66.60

4.21

78.50

4.38

81.10

40.20

3.72
9.10

2.31

4.10

1.63

63.70

22.10

3.14
5

5

5

5

5

5

5 


\section{STD ERROR MAXIMUM MINIMUM \\ OF MEAN \\ VALUE \\ VALUE}

$\mathbf{N}$

HIGH RAINFALL FY-1989 UTR CK ABOVE

$\begin{array}{lrrrrr}\text { TSS } & 5.69 & 0.55 & 7.80 & 3.10 & 10 \\ \text { LTSS } & 1.87 & 0.09 & 2.17 & 1.41 & 10 \\ \text { TURB } & 2.39 & 0.47 & 4.00 & 0.60 & 10 \\ \text { LTURB } & 1.12 & 0.16 & 1.61 & 0.47 & 10 \\ \text { PTSASH } & 51.95 & 1.51 & 59.20 & 41.00 & 10 \\ \text { SCOND } & 16.89 & 0.57 & 19.40 & 14.80 & 10 \\ \text { LSCOND } & 2.88 & 0.03 & 3.02 & 2.76 & 10\end{array}$

HIGH RAINFALL FY-1989 TINKER CK

$\begin{array}{lrrrrr} & & & & & \\ \text { TSS } & 14.24 & 3.32 & 35.30 & 4.10 & 10 \\ \text { LTSS } & 2.54 & 0.20 & 3.59 & 1.63 & 10 \\ \text { TURB } & 4.52 & 0.71 & 9.60 & 2.20 & 10 \\ \text { LTURB } & 1.64 & 0.12 & 2.36 & 1.16 & 10 \\ \text { PTSASH } & 53.53 & 1.10 & 60.90 & 48.70 & 10 \\ \text { SCOND } & 30.99 & 1.40 & 38.30 & 26.30 & 10 \\ \text { LSCOND } & 3.46 & 0.04 & 3.67 & 3.31 & 10\end{array}$

HIGH RAINFALL FY-1989 MCQUEEN BR

$\begin{array}{lrrrrr} & & & & \\ \text { TSS } & 217.39 & 62.32 & 42.50 & 92.00 & 10 \\ \text { LTSS } & 5.16 & 0.20 & 6.61 & 4.53 & 10 \\ \text { TURB } & 254.00 & 49.38 & 680.00 & 140.00 & 10 \\ \text { LTURB } & 5.43 & 0.14 & 6.52 & 4.95 & 10 \\ \text { PTSASH } & 88.73 & 0.84 & 92.50 & 82.60 & 10 \\ \text { SCOND } & 54.99 & 5.90 & 95.90 & 41.40 & 10 \\ \text { LSCOND } & 3.98 & 0.09 & 4.57 & 3.75 & 10\end{array}$

HIGH RAINFALL FY-1989 UTR CK MID.

$\begin{array}{lrrrrr}\text { TSS } & 26.00 & 13.14 & 76.00 & 6.10 & \mathbf{5} \\ \text { LTSS } & 2.87 & 0.44 & 4.34 & 1.96 & \mathbf{5} \\ \text { TURB } & 31.90 & 16.27 & 89.00 & 3.20 & \mathbf{5} \\ \text { LTURB } & 2.84 & 0.62 & 4.50 & 1.44 & 5 \\ \text { PTSASH } & 70.64 & 5.40 & 82.90 & 57.40 & 5 \\ \text { SCOND } & 25.48 & 1.20 & 28.70 & 23.30 & \mathbf{5} \\ \text { LSCOND } & 3.27 & 0.04 & 3.39 & 3.19 & \mathbf{5}\end{array}$

HIGH RAINFALL FY-1989 CROUCH BR

$\begin{array}{lrrrrr}\text { TSS } & 246.32 & 59.55 & 466.50 & 119.70 & 5 \\ \text { LTSS } & 5.41 & 0.22 & 6.15 & 4.79 & 5 \\ \text { TURB } & 362.00 & 58.77 & 560.00 & 210.00 & 5 \\ \text { LTURB } & 5.84 & 0.16 & 6.33 & 5.35 & 5 \\ \text { PTSASH } & 91.22 & 0.65 & 92.90 & 89.60 & 5 \\ \text { SCOND } & 91.32 & 11.19 & 126.00 & 63.40 & 5 \\ \text { LSCOND } & 4.50 & 0.12 & 4.84 & 4.17 & \mathbf{5}\end{array}$


VARIABLE MEAN

STD ERROR
OF MEAN

HIGH RAINFALL

$\begin{array}{lrr}\text { TSS } & 39.16 & 17.33 \\ \text { LTSS } & 3.24 & 0.50 \\ \text { TURB } & 45.82 & 21.33 \\ \text { LTURB } & 3.19 & 0.64 \\ \text { PTSASH } & 75.04 & 5.12 \\ \text { SCOND } & 25.98 & 1.12 \\ \text { LSCOND } & 3.29 & 0.04\end{array}$

HIGH RAINFALL FY-1990

$\begin{array}{lrr}\text { TSS } & 14.80 & 0.70 \\ \text { LTSS } & 2.76 & 0.04 \\ \text { TURB } & 3.55 & 0.15 \\ \text { LTURB } & 1.51 & 0.03 \\ \text { PTSASH } & 49.10 & 7.10 \\ \text { SCOND } & 21.60 & 3.50 \\ \text { LSCOND } & 3.11 & 0.16\end{array}$

HIGH RAINFALL FY-1990

TSS

LTSS

TURB

LTURB

PTSASH

SCOND

LSCOND
36.25

3.34

9.05

2.30

59.80

33.85

3.54
24.35

0.78

0.75

0.07

8.00

5.45

0.16
MAXIMUM

VALUE
MINIMUM

VALUE

$\mathbf{N}$

UTR CK BELOW

$\begin{array}{rrr}93.60 & 5.50 & 5 \\ 4.55 & 1.87 & 5 \\ 105.00 & 2.80 & 5 \\ 4.66 & 1.34 & 5 \\ 85.00 & 56.40 & 5 \\ 29.30 & 23.90 & 5 \\ 3.41 & 3.21 & 5\end{array}$

UTR CK ABOVE

$\begin{array}{rrr}15.50 & 14.10 & 2 \\ 2.80 & 2.71 & 2 \\ 3.70 & 3.40 & 2 \\ 1.55 & 1.48 & 2 \\ 56.20 & 42.00 & 2 \\ 25.10 & 18.10 & 2 \\ 3.26 & 2.95 & 2\end{array}$

HIGH RAINFALL FY-1990 MCQUEEN BR

$\begin{array}{lrr}\text { TSS } & 571.10 & 250.70 \\ \text { LTSS } & 6.24 & 0.47 \\ \text { TURB } & 455.00 & 180.00 \\ \text { LTURB } & 6.04 & 0.42 \\ \text { PTSASH } & 89.15 & 0.25 \\ \text { SCOND } & 30.90 & 2.40 \\ \text { LSCOND } & 3.46 & 0.08\end{array}$

HIGH RAINFALL FY-1990

TINKER CK

$\begin{array}{rrr}60.60 & 11.90 & 2 \\ 4.12 & 2.56 & 2 \\ 9.80 & 8.30 & 2 \\ 2.38 & 2.23 & 2 \\ 67.80 & 51.80 & 2 \\ 39.30 & 28.40 & 2 \\ 3.70 & 3.38 & 2\end{array}$

$\begin{array}{rrr}821.80 & 320.40 & 2 \\ 6.71 & 5.77 & 2 \\ 635.00 & 275.00 & 2 \\ 6.46 & 5.62 & 2 \\ 89.40 & 88.90 & 2 \\ 33.30 & 28.50 & 2 \\ 3.54 & 3.38 & 2\end{array}$

\begin{tabular}{lrl} 
& & HIGH RAINFALL \\
\hline TSS & 40.20 & - \\
LTSS & 3.72 & - \\
TURB & 60.00 & - \\
LTURB & 4.11 & - \\
PTSASH & 81.80 & - \\
SCOND & 76.10 & - \\
LSCOND & 4.35 & -
\end{tabular}

UTR CK MID.

$\begin{array}{rrr}40.20 & 40.20 & 1 \\ 3.72 & 3.72 & 1 \\ 60.00 & 60.00 & 1 \\ 4.11 & 4.11 & 1 \\ 81.80 & 81.80 & 1 \\ 76.10 & 76.10 & 1 \\ 4.35 & 4.35 & 1\end{array}$


80

VARIABLE MEAN

\section{STD ERROR \\ OF MEAN}

MAXIMUM
VALUE

MINIMUM

VALUE

$\mathbf{N}$

HIGH RAINFALL FY- 1990

$\begin{array}{lrl}\text { TSS } & 401.00 & -- \\ \text { LTSS } & 6.00 & -- \\ \text { TURB } & 490.00 & -- \\ \text { LTURB } & 6.20 & -- \\ \text { PTSASH } & 91.30 & -- \\ \text { SCOND } & 32.90 & -- \\ \text { LSCOND } & 3.52 & --\end{array}$

CROUCH BR

401.00

6.00

$401.00 \quad 1$

490.00

6.20

91.30

32.90

3.52

$\begin{array}{rr}4.00 & 1 \\ 490.00 & 1 \\ 6.20 & 1 \\ 91.30 & 1 \\ 32.90 & 1 \\ 3.52 & 1\end{array}$

HIGH RAINFALL

TSS

LTSS

TURB

LTURB

PTSASH

SCOND

LSCOND
180.30

5.20

240.00

5.48

90.20

38.90

3.69
FY-1990

--

HIGH RAINFALL

\subsection{2}

2.04

1.44

0.84

58.52

20.48

3.01

\subsection{6}

0.13

0.32

0.14

1.03

3.27

0.14

HIGH RAINFALL FY-1991

TSS

LTSS

TURB

LTURB

PTSASH

SCOND

LSCOND
10.00

2.20

1.69

0.93

54.65

30.88

3.45
3.02

0.28

0.41

0.15

2.54

2.65

0.08

HIGH RAINFALL FY-1991

TSS

LTSS

TURB

LTURB

PTSASH

SCOND

LSCOND
158.85

4.42

122.03

4.16

82.27

35.13

3.54
65.20

0.58

48.15

0.60

1.89

4.65

0.13
UTR CK BELOW

180.30

5.20

240.00

5.48

90.20

38.90

3.69
180.30

5.20

240.00

5.48

90.20

38.90

3.69
4.14

1.64

0.49

0.40

54.60

14.70

2.75

1
1
1
1
1
1
1

UTR CK ABOVE

10.34
2.43
2.30
1.19
61.80
32.10
3.50

TINKER CK

20.65
3.08
3.00
1.39
65.30
41.80
3.76

2.70

1.31

0.60

0.47

48.30

25.20

3.27

MCQUEEN BR

402.10

6.00

290.00

5.67

87.20

50.50

3.94
13.69

2.69

5.20

1.82

75.70

21.90

3.13
6

6

6

6

6

6

6 
VARIABLE MEAN

STD ERROR

MAXIMUM

MINIMUM

VALUE

VALUE

$\mathbf{N}$

HIGH RAINFALL FY-1991 UTR CK MID

$\begin{array}{lrr}\text { TSS } & 44.35 & 35.72 \\ \text { LTSS } & 3.06 & 0.87 \\ \text { TURB } & 51.09 & 49.46 \\ \text { LTURB } & 2.27 & 1.39 \\ \text { PTSASH } & 67.77 & 10.55 \\ \text { SCOND } & 25.63 & 4.73 \\ \text { LSCOND } & 3.25 & 0.17\end{array}$

115.70

4.76

5.55

3

150.00

1.88

3

49.46

1.39

5.02

0.68

88.80

35.10

0.52

55.80

20.70

3.08

3

3

4.73

3.59

3

3

3

HIGH RAINFALL FY-1991 CROUCH BR

\begin{tabular}{|c|c|c|}
\hline TSS & 130.08 & 103.9 \\
\hline LTSS & 3.97 & 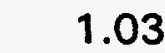 \\
\hline TURB & 152.67 & 115 \\
\hline $\begin{array}{l}\text { LTURB } \\
\text { PTSASH }\end{array}$ & $\begin{array}{r}4.01 \\
85.47\end{array}$ & \\
\hline SCOND & 60.30 & 2 \\
\hline LSCOND & 3.95 & \\
\hline
\end{tabular}

HIGH RAINFALL FY-1991

$\begin{array}{lrr}\text { TSS } & 30.79 & 20.15 \\ \text { LTSS } & 3.03 & 0.66 \\ \text { TURB } & 25.94 & 24.04 \\ \text { LTURB } & 2.11 & 1.12 \\ \text { PTSASH } & 71.10 & 8.32 \\ \text { SCOND } & 26.50 & 5.45 \\ \text { LSCOND } & 3.28 & 0.18\end{array}$

337.00

5.82

8.53

3

380.00

2.25

3

5.94

5.00

3

92.20

1.79

3

95.20

76.90

3

4.57

21.60

3

3.12

3

UTR CK BELOW

$\begin{array}{rrr}\text { TR CK BELOW } & & \\ 70.80 & 6.68 & 3 \\ 4.27 & 2.04 & 3 \\ 74.00 & 0.92 & 3 \\ 4.32 & 0.65 & 3 \\ 86.80 & 58.50 & 3 \\ 37.40 & 21.00 & 3 \\ 3.65 & 3.09 & 3\end{array}$

HIGH RAINFALL FY-1992 UTR CK ABOVE

$\begin{array}{lrl}\text { TSS } & 7.06 & 1.08 \\ \text { LTSS } & 2.04 & 0.12 \\ \text { TURB } & 1.14 & 0.31 \\ \text { LTURB } & 0.70 & 0.13 \\ \text { PTSASH } & 55.51 & 1.16 \\ \text { SCOND } & 17.79 & 0.67 \\ \text { LSCOND } & 2.93 & 0.04\end{array}$

13.20
2.65
2.70
1.31
59.20
19.90
3.04

4.75

8

1.75

8

0.48

0.39

49.00

8

HIGH RAINFALL FY-1992

$\begin{array}{lrr}\text { TSS } & 8.98 & 2.23 \\ \text { LTSS } & 2.18 & 0.18 \\ \text { TURB } & 1.23 & 0.25 \\ \text { LTURB } & 0.76 & 0.12 \\ \text { PTSASH } & 47.81 & 2.53 \\ \text { SCOND } & 27.05 & 1.08 \\ \text { LSCOND } & 3.33 & 0.04\end{array}$

2.23

0.18

0.25

0.12

2.53

15.30

2.79

3
3
3
3
3
3


82

VARIABLE MEAN

\section{STD ERROR \\ OF MEAN}

MAXIMUM

VALUE
MINIMUM

VALUE

$\mathbf{N}$

HIGH RAINFALL FY-1992 MCQUEEN BR

$\begin{array}{lrrrrr}\text { TSS } & 16.69 & 2.46 & 30.30 & 9.62 & 8 \\ \text { LTSS } & 2.81 & 0.13 & 3.44 & 2.36 & 8 \\ \text { TURB } & 18.09 & 6.42 & 53.00 & 4.00 & 8 \\ \text { LTURB } & 2.56 & 0.33 & 3.99 & 1.61 & 8 \\ \text { PTSASH } & 82.44 & 1.98 & 87.90 & 71.40 & 8 \\ \text { SCOND } & 45.18 & 2.29 & 58.30 & 36.30 & 8 \\ \text { LSCOND } & 3.82 & 0.05 & 4.08 & 3.62 & 8\end{array}$

HIGH RAINFALL FY-1992 UTR CK MID

$\begin{array}{lrrrrr}\text { TSS } & 8.75 & 0.72 & 10.80 & 7.71 & 4 \\ \text { LTSS } & 2.27 & 0.07 & 2.47 & 2.16 & 4 \\ \text { TURB } & 1.63 & 0.49 & 2.70 & 0.67 & 4 \\ \text { LTURB } & 0.91 & 0.19 & 1.31 & 0.51 & 4 \\ \text { PTSASH } & 55.63 & 1.78 & 60.50 & 52.20 & 4 \\ \text { SCOND } & 23.55 & 0.61 & 25.30 & 22.60 & 4 \\ \text { LSCOND } & 3.20 & 0.02 & 3.27 & 3.16 & 4\end{array}$

HIGH RAINFALL FY-1992 CROUCH BR

$\begin{array}{lrr}\text { TSS } & 44.08 & 15.40 \\ \text { LTSS } & 3.67 & 0.29 \\ \text { TURB } & 67.75 & 20.80 \\ \text { LTURB } & 4.12 & 0.25 \\ \text { PTSASH } & 86.70 & 2.08 \\ \text { SCOND } & 84.83 & 9.22 \\ \text { LSCOND } & 4.44 & 0.10\end{array}$

89.90

4.51

130.00

4.88

90.80

110.00

4.71

$\begin{array}{rr}23.50 & 4 \\ 3.20 & 4 \\ 43.00 & 4 \\ 3.78 & 4 \\ 81.10 & 4 \\ 67.70 & 4 \\ 4.23 & 4\end{array}$

HIGH RAINFALL FY-1992 UTR CK BELOW

$\begin{array}{lrrrrr}\text { TSS } & 9.05 & 0.82 & 11.40 & 7.70 & 4 \\ \text { LTSS } & 2.30 & 0.08 & 2.52 & 2.16 & 4 \\ \text { TURB } & 1.99 & 0.59 & 3.60 & 0.74 & 4 \\ \text { LTURB } & 1.03 & 0.20 & 1.53 & 0.55 & 4 \\ \text { PTSASH } & 57.55 & 2.53 & 64.50 & 52.40 & 4 \\ \text { SCOND } & 22.88 & 0.80 & 24.80 & 21.10 & 4 \\ \text { LSCOND } & 3.17 & 0.03 & 3.25 & 3.10 & 4\end{array}$

HIGH RAINFALL FY-1993 UTR CK ABOVE

$\begin{array}{lrr}\text { TSS } & 21.46 & 7.99 \\ \text { LTSS } & 2.84 & 0.25 \\ \text { TURB } & 27.30 & 13.57 \\ \text { LTURB } & 2.85 & 0.34 \\ \text { PTSASH } & 65.20 & 4.15 \\ \text { SCOND } & 17.73 & 0.59 \\ \text { LSCOND } & 2.93 & 0.03\end{array}$

75.70
4.34
120.00
4.80
86.70
20.00
3.04

6.53

8

2.02

8

5.00

1.79

8

54.90

8

0.03

15.00

8

8

$2.77 \quad 8$


STD ERR OF ME
MAXIMUM

VALUE
MINIMUM

VALUE

$\mathbf{N}$

HIGH RAINFAL

$\begin{array}{lr}\text { TSS } & 10.17 \\ \text { LTSS } & 2.34 \\ \text { TURB } & 8.74\end{array}$

EY-1993
12
14
16
12
259
171
0.36

TINKER CK

19.94

3.04

5.66

1.90

8

13.00

2.64

LTURB

2.23

PTSASH

51.91

62.40

24.86

34.80

LSCOND

3.24

3.58

4.80

1.76

39.10

20.70

3.08

8

8

8

8

8

8

HIGH RAINFALL FY-1993 MCQUEEN BR

$\begin{array}{lrr} & & \\ \text { TSS } & 93.56 & 50.26 \\ \text { LTSS } & 3.66 & 0.50 \\ \text { TURB } & 122.63 & 61.06 \\ \text { LTURB } & 4.02 & 0.48 \\ \text { PTSASH } & 84.75 & 2.36 \\ \text { SCOND } & 34.21 & 2.92 \\ \text { LSCOND } & 3.54 & 0.08\end{array}$

$\begin{array}{rrr}\text { MCQUEEN BR } & & \\ 422.20 & 8.62 & 8 \\ 6.05 & 2.26 & 8 \\ 510.00 & 13.00 & 8 \\ 6.24 & 2.64 & 8 \\ 93.70 & 76.40 & 8 \\ 45.40 & 25.00 & 8 \\ 3.83 & 3.26 & 8\end{array}$

HIGH RAINFALL FY-1993 UTR CK MID

$\begin{array}{lrr} & & \\ \text { TSS } & 15.31 & 4.23 \\ \text { LTSS } & 2.70 & 0.24 \\ \text { TURB } & 18.75 & 7.11 \\ \text { LTURB } & 2.83 & 0.30 \\ \text { PTSASH } & 66.60 & 6.22 \\ \text { SCOND } & 22.48 & 2.28 \\ \text { LSCOND } & 3.14 & 0.10\end{array}$

HIGH RAINFALL FY-1993 CROUCH BR

$\begin{array}{lrr}\text { TSS } & 116.63 & 68.78 \\ \text { LTSS } & 4.18 & 0.65 \\ \text { TURB } & 160.25 & 90.94 \\ \text { LTURB } & 4.51 & 0.66 \\ \text { PTSASH } & 83.53 & 7.82 \\ \text { SCOND } & 52.90 & 11.46 \\ \text { LSCOND } & 3.92 & 0.21\end{array}$

HIGH RAINFALL FY-1993 UTR CK BELOW

$\begin{array}{lrr}\text { TSS } & 66.93 & 53.12 \\ \text { LTSS } & 3.31 & 0.73 \\ \text { TURB } & 83.00 & 65.82 \\ \text { LTURB } & 3.50 & 0.75 \\ \text { PTSASH } & 76.55 & 5.93 \\ \text { SCOND } & 22.00 & 2.12 \\ \text { LSCOND } & 3.12 & 0.09\end{array}$

226.00

5.42

7.37

8.81

2.28

4

3.35

10.00

2.40

3.71

57.60

18.20

2.95

8

84.70
28.00
3.37

313.90
5.75

17.39

2.91

420.00

19.00

3.00

94.60

60.70

32.90

3.52

4

4

4

4

4

4

83.50

4.44

2.12

9.00

2.30

5.64

61.20

18.10

27.00

2.95

4
4
4
4
4
4
4

3.33

4
4
4
4
4
4
4




\section{APPENDIX B}

PROBABILITY LEVELS FOR TESTS OF MAIN EFFECTS

AND INTERACTION EFFECTS IN ANALYSIS OF VARIANCE MODEL 
P VALUES OF ANOVA MAIN EFFECTS

NO RAIN

Log TSS

Log Specific

Main Effect

Conductivity

Log Percent Log Turbidity Ash

$\begin{array}{lllll}\text { Location } & 0.0001 & 0.0001 & 0.0001 & 0.0001 \\ \text { Construction } & 0.0001 & 0.0001 & 0.0001 & 0.0001 \\ \text { Loc }{ }^{\circ} \text { Con } & 0.0001 & 0.03 & 0.0001 & 0.0001 \\ \quad R^{2}= & 0.47 & 0.58 & 0.68 & 0.61\end{array}$

LOW RAIN*

Main Effect

Log TSS

Log Specific

Log Percent

Log Turbidity Conductivity

Ash

\begin{tabular}{lllll} 
Log Rain & 0.48 & 0.003 & 0.61 & 0.0007 \\
Location & 0.10 & 0.0001 & 0.0001 & 0.0004 \\
Construction & 0.84 & 0.03 & 0.39 & 0.07 \\
Log Rain ${ }^{\text {Loc }}$ & 0.12 & 0.99 & 0.73 & 0.21 \\
Log Rain Con & 0.23 & 0.004 & 0.03 & 0.96 \\
Loc*Con & 0.67 & 0.27 & 0.49 & 0.89 \\
\multicolumn{1}{c}{$\mathbf{R}^{2}=$} & 0.45 & 0.78 & 0.78 & 0.62
\end{tabular}

HIGH RAIN*

Main Effect

Log TSS

Log Specific

Log Percent

Ash

Log Turbidity Conductivity

$\begin{array}{lll}0.61 & 0.0001 & 0.0001 \\ 0.0001 & 0.0001 & 0.0001 \\ 0.67 & 0.28 & 0.04 \\ 0.0015 & 0.0001 & 0.01 \\ 0.83 & 0.40 & 0.11 \\ 0.38 & 0.10 & 0.80\end{array}$

Log Rain

0.0001

0.0001

0.08

0.11

0.22

0.64

0.38

0.10

0.80

$\mathrm{R}^{2}=$

0.63

0.70

0.82

0.72

* "RAIN" is for amount of precipitation 24 hours prior to sampling. 
APPENDIX C

DWPF Means of Water Quality Variables for each

Construction Period from FY-1983 to FY-1993

(summarized by rainfall class and location) 
MEANS BY CONSTRUCTION PERIOD

LOCATION

NO RAIN - - TSS - - BEFORE CONSTRUCTION - MEAN

STD. ERR.

$\mathrm{N}$

UTR - ABOVE

3.42

3.82

0.24

33

TINKER CREEK

5.38

0.36

31

MCQUEEN BRANCH

4.04

0.31

22

UTR - MIDDLE

CROUCH BRANCH

$--$

0.42

17

UTR - BELOW

4.10

0.49

LOCATION

NO RAIN

N - PERCEN

T ASH - - BEFORE CONSTRUCTION MEAN STD. ERR.

$\mathrm{N}$

UTR - ABOVE

TINKER CREEK

MCQUEEN BRANCH

UTR - MIDDLE

CROUCH BRANCH

UTR- BELOW
44.18

42.54

58.64

47.39

$-$

45.73
1.53

1.52

1.75

1.13

$-$

2.33
33

$-$

17
OCATION
UTR - ABOVE

TINKER CREEK

MCQUEEN BRANCH

UTR - MIDDLE

CROUCH BRANCH

UTR-BELOW
26.44

49.55

58.89

36.50

36.60
1.04

2.04

3.90

1.99

$-$

2.05
33

31

22

17

17 
MEANS BY CONSTRUCTION PERIOD

\begin{tabular}{|c|c|c|c|}
\hline \multirow{2}{*}{ LOCATION } & \multirow{2}{*}{ TSS - - } & & \multirow{2}{*}{$N$} \\
\hline & & STD. ERR. & \\
\hline $\begin{array}{l}\text { UTR - ABOVE } \\
\text { TINKER CREEK } \\
\text { MCQUEEN BRANCH } \\
\text { UTR-MIDDLE } \\
\text { CROUCH BRANCH } \\
\text { UTR - BELOW }\end{array}$ & $\begin{array}{l}6.01 \\
5.42 \\
18.26 \\
6.25 \\
88.38 \\
6.12\end{array}$ & $\begin{array}{l}0.85 \\
0.61 \\
4.84 \\
0.68 \\
19.83 \\
0.58\end{array}$ & $\begin{array}{l}66 \\
66 \\
48 \\
33 \\
16 \\
33\end{array}$ \\
\hline LOCATION & $\begin{array}{l}\text { RBIDITY } \\
\text { MEAN }\end{array}$ & $\begin{array}{l}\text { CONSTRUC } \\
\text { STD. ERR. }\end{array}$ & $\mathrm{N}$ \\
\hline $\begin{array}{l}\text { UTR - ABOVE } \\
\text { TINKER CREEK } \\
\text { MCQUEEN BRANCH } \\
\text { UTR- MIDDLE } \\
\text { CROUCH BRANCH } \\
\text { UTR - BELOW }\end{array}$ & $\begin{array}{l}3.36 \\
3.62 \\
25.62 \\
3.99 \\
158.22 \\
4.19\end{array}$ & $\begin{array}{l}0.37 \\
0.47 \\
8.58 \\
0.45 \\
34.27 \\
0.40\end{array}$ & $\begin{array}{l}66 \\
66 \\
48 \\
33 \\
16 \\
33\end{array}$ \\
\hline
\end{tabular}

$\begin{array}{llll}\text { LOCATION } & \text { MEAN } & \text { STD. ERR. } & \text { N } \\ & & & 66 \\ \text { UTR - ABOVE } & 46.60 & 1.39 & 66 \\ \text { TINKER CREEK } & 46.82 & 1.13 & 48 \\ \text { MCQUEEN BRANCH } & 74.52 & 1.11 & 33 \\ \text { UTR - MIDDLE } & 50.38 & 1.59 & 16 \\ \text { CROUCH BRANCH } & 86.16 & 1.25 & 33 \\ \text { UTR - BELOW } & 49.77 & 1.47 & \end{array}$

\begin{tabular}{lccc}
\multicolumn{2}{c}{ - - - NO RAIN - - SPECIFIC CONDUCTANCE - - DURING CONSTRUCT } \\
LOCATION & MEAN & STD. ERR. & N \\
& & & 66 \\
UTR - ABOVE & 16.38 & 1.89 & 66 \\
TINKER CREEK & 24.74 & 7.17 & 48 \\
MCQUEEN BRANCH & 55.93 & 1.30 & 33 \\
UTR- MIDDLE & 19.60 & 3.80 & 16 \\
CROUCH BRANCH & 93.37 & 1.07 & 33 \\
UTR - BELOW & 20.31 & &
\end{tabular}


MEANS BY CONSTRUCTION PERIOD

\begin{tabular}{llll} 
& $\ldots$. . NO RAIN - - TSS - & AFTER CONSTRUCTION - - & \\
LOCATION & MEAN & STD. ERR. & N \\
& & & \\
\hline UTR - ABOVE & 4.63 & 0.35 & 30 \\
TINKER CREEK & 7.03 & 0.72 & 30 \\
MCOUEEN BRANCH & 5.99 & 0.96 & 30 \\
UTR - MIDDLE & 5.96 & 0.62 & 15 \\
CROUCH BRANCH & 19.75 & 4.40 & 15 \\
UTR- BELOW & 6.47 & 0.62 & 15
\end{tabular}

$\begin{array}{llll}\text { LOCATION } & \text { - - NO RAIN - - TURBIDITY - A AFTER CONSTRUCTION - - } \\ & \text { MEAN } & \text { STD. ERR. } & \text { N } \\ \text { UTR - ABOVE } & 1.91 & 0.33 & 30 \\ \text { TINKER CREEK } & 2.77 & 0.36 & 30 \\ \text { MCOUEEN BRANCH } & 5.15 & 0.84 & 30 \\ \text { UTR - MIDDLE } & 2.33 & 0.48 & 15 \\ \text { CROUCH BRANCH } & 31.57 & 7.88 & 15 \\ \text { UTR - BELOW } & 2.16 & 0.50 & 15\end{array}$

\begin{tabular}{lccc}
\multicolumn{2}{c}{-- - NO RAIN - - PERCENT ASH - - AFTER CONSTRUCTION - - } \\
LOCATION & MEAN & STD. ERR. & N \\
& & & 30 \\
UTR - ABOVE & 55.05 & 1.51 & 30 \\
TINKER CREEK & 54.04 & 1.28 & 30 \\
MCQUEEN BRANCH & 72.69 & 1.42 & 15 \\
UTR - MIDDLE & 52.79 & 1.94 & 15 \\
CROUCH BRANCH & 80.41 & 1.87 & 15 \\
UTR - BELOW & 57.05 & 1.27 &
\end{tabular}

$\begin{array}{llll}\text { LOCATION } & \text { RAIN - - SPECIFIC CONDUCTANCE - - AFTER CONSTRUCTIO } \\ & \text { MEAN } & \text { STD. ERR. } & \text { N } \\ \text { UTR - ABOVE } & 14.67 & 0.15 & 30 \\ \text { TINKER CREEK } & 29.09 & 0.83 & 30 \\ \text { MCOUEEN BRANCH } & 74.29 & 11.92 & 30 \\ \text { UTR - MIDDLE } & 20.45 & 0.52 & 15 \\ \text { CROUCH BRANCH } & 101.71 & 10.04 & 15 \\ \text { UTR - BELOW } & 21.04 & 0.55 & 15\end{array}$


MEANS BY CONSTRUCTION PERIOD

\begin{tabular}{|c|c|c|c|}
\hline LOCATION & MEAN & STD. ERR. & $\mathbf{N}$ \\
\hline UTR - ABOVE & 4.85 & 0.41 & 10 \\
\hline TINKER CREEK & 5.20 & 0.63 & 8 \\
\hline MCQUEEN BRANCH & 6.93 & 0.75 & 7 \\
\hline UTR - MIDDLE & 6.16 & 0.76 & 5 \\
\hline CROUCH BRANCH & -. & -- & -- \\
\hline UTR - BELOW & 6.15 & 0.73 & 5 \\
\hline
\end{tabular}

\begin{tabular}{lccc}
\multicolumn{2}{c}{ - - - LOW RAIN - - PERCENT ASH - - BEFORE CONSTRUCTION - - } \\
LOCATION & MEAN & STD. ERR. & N \\
& & & 10 \\
UTR - ABOVE & 47.73 & 5.67 & 8 \\
TINKER CREEK & 42.14 & 3.16 & 7 \\
MCOUEEN BRANCH & 61.00 & 3.19 & 5 \\
UTR - MIDDLE & 43.81 & 3.16 & - \\
CROUCH BRANCH & -- & - & 5 \\
UTR - BELOW & 44.41 & 1.73 &
\end{tabular}

- . - LOW RAIN - - SPECIFIC CONDUCTANCE - - BEFORE CONSTRUCTION - LOCATION MEAN STD. ERR.

$\mathbf{N}$

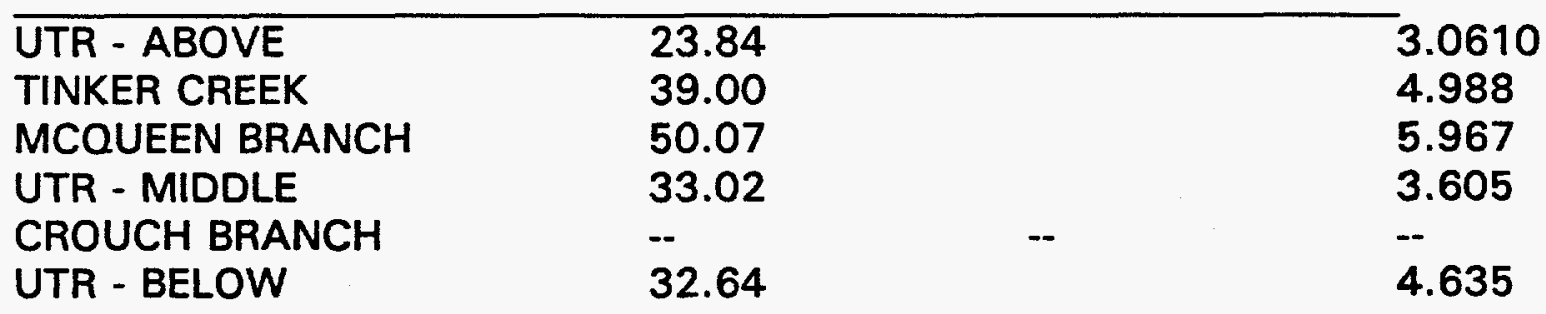


MEANS BY CONSTRUCTION PERIOD

-- - - LOW RAIN - - TSS- - DURING CONSTRUCTION - --

\begin{tabular}{llll} 
LOCATION & MEAN & STD. ERR. & N \\
\cline { 1 - 2 } UTR - ABOVE & & & \\
TINKER CREEK & 6.31 & 0.60 & 16 \\
MCQUEEN BRANCH & 8.53 & 0.96 & 16 \\
UTR- MIDDLE & 29.20 & & 7.6213 \\
CROUCH BRANCH & 8.88 & 1.22 & 8 \\
UTR - BELOW & 50.12 & & 10.886 \\
UT & 8.92 & 1.27 & 8
\end{tabular}

\begin{tabular}{llll}
\multicolumn{2}{c}{- - - LOW RAIN - - TURBIDITY - - DURING CONSTRUCTION - - } \\
LOCATION & MEAN & STD. ERR. & N \\
& & & \\
UTR - ABOVE & 3.27 & 0.37 & 16 \\
TINKER CREEK & 3.71 & 0.30 & 16 \\
MCQUEEN BRANCH & 42.08 & 13.65 & 13 \\
UTR - MIDDLE & 4.13 & 0.45 & 8 \\
CROUCH BRANCH & 98.67 & 23.80 & 6 \\
UTR - BELOW & 4.65 & 0.61 & 8
\end{tabular}

\begin{tabular}{lccc}
\multicolumn{2}{c}{$-\ldots$ - LOW RAIN - - PERCENT ASH - - DURING CONSTRUCTION - . - } \\
LOCATION & MEAN & STD. ERR. & N \\
& & & 16 \\
UTR - ABOVE & 55.09 & 2.36 & 16 \\
TINKER CREEK & 52.75 & 2.51 & 13 \\
MCQUEEN BRANCH & 80.81 & 2.63 & 8 \\
UTR - MIDDLE & 57.29 & 3.03 & 6 \\
CROUCH BRANCH & 87.03 & 1.43 & 8 \\
UTR - BELOW & 58.31 & 2.57 &
\end{tabular}

\begin{tabular}{lllc} 
LOCATION & \multicolumn{3}{c}{ - - LOW RAIN - - SPECIFIC CONDUCTANCE - - DURING CONSTRUC } \\
& MEAN & STD. ERR. & N \\
& & & 16 \\
UTR - ABOVE & 15.79 & 0.44 & 16 \\
TINKER CREEK & 28.42 & 1.48 & 13 \\
MCQUEEN BRANCH & 58.57 & 7.31 & 8 \\
UTR - MIDDLE & 19.87 & 2.00 & 6 \\
CROUCH BRANCH & 90.02 & 7.14 & 8 \\
UTR - BELOW & 20.82 & 2.30 &
\end{tabular}


MEANS BY CONSTRUCTION PERIOD

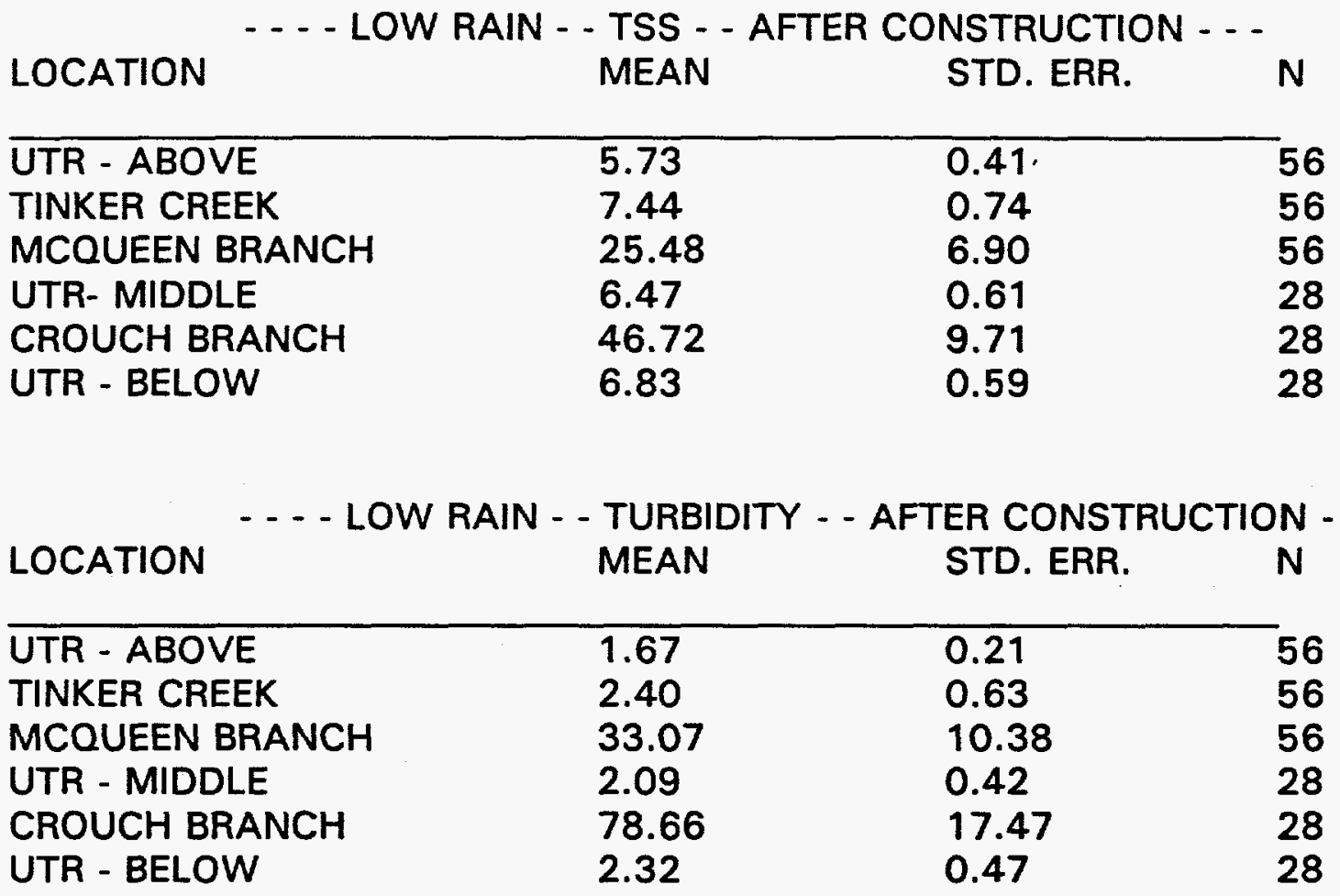

\begin{tabular}{|c|c|c|c|}
\hline LOCATION & MEAN & STD. ERR. & $N$ \\
\hline $\begin{array}{l}\text { UTR - ABOVE } \\
\text { TINKER CREEK } \\
\text { MCQUEEN BRANCH } \\
\text { UTR - MIDDLE } \\
\text { CROUCH BRANCH }\end{array}$ & $\begin{array}{l}53.85 \\
50.81 \\
77.54 \\
54.06 \\
81.55\end{array}$ & $\begin{array}{l}0.61 \\
0.79 \\
1.06 \\
0.88 \\
1.70\end{array}$ & $\begin{array}{l}56 \\
56 \\
56 \\
28 \\
28\end{array}$ \\
\hline UTR - BELOW & 56.12 & 0.77 & 28 \\
\hline
\end{tabular}

- - - LOW RAIN - - SPECIFIC CONDUCTANCE - - AFTER CONSTRUCTION - LOCATION MEAN STD. ERR.

$\mathrm{N}$

\begin{tabular}{llll}
\hline UTR - ABOVE & 16.63 & 0.34 & 56 \\
TINKER CREEK & 28.66 & 0.63 & 56 \\
MCOUEEN BRANCH & 68.59 & 10.50 & 56 \\
UTR - MIDDLE & 21.52 & 0.29 & 28 \\
CROUCH BRANCH & 91.28 & 4.43 & 28 \\
UTR- BELOW & 22.29 & 0.31 & 28
\end{tabular}


MEANS BY CONSTRUCTION PERIOD

LOCATION - - - HIGH RAIN - - TSS - - BEFORE CONSTRUCTION - -

UTR - ABOVE

TINKER CREEK

MCOUEEN BRANCH

UTR - MIDDLE

CROUCH BRANCH

UTR - BELOW MEAN

STD. ERR.
N

$\begin{array}{lll}9.36 & 1.62 & 10 \\ 11.15 & 2.09 & 9 \\ 59.04 & 16.31 & 6 \\ 28.59 & 12.81 & 5 \\ -- & -- & -- \\ 39.01 & 18.60 & 5\end{array}$

LOCATION

- . - HIGH RAIN - PERC

\section{MEAN}

53.04

52.00

76.81

63.25

--

CROUCH BRANCH

UTR - BELOW

UTR - ABOVE
TINKER CREEK
MCQUEEN BRANCH
UTR - MIDDLE
CROUCH BRANCH
UTR - BELOW

UTR - ABOVE

60.64
ASH

(2)

(n)

$\begin{array}{cc}0.74 & 10 \\ 4.96 & 9 \\ 3.06 & 6 \\ 4.27 & 5 \\ --.- & \\ 9.35 & 5\end{array}$

- - - HIGH RAIN - - SPECIFIC
LOCATION

C CONDUCTANCE MEAN STD. ERR.

\begin{tabular}{llll}
\hline UTR - ABOVE & 29.15 & 1.62 & 10 \\
TINKER CREEK & 44.37 & 1.05 & 9 \\
MCQUEEN BRANCH & 51.28 & 6.23 & 6 \\
UTR - MIDDLE & 34.80 & 3.19 & 5 \\
CROUCH BRANCH & -- & -- & - \\
UTR- BELOW & 34.20 & 2.50 & 5
\end{tabular}


MEANS BY CONSTRUCTION PERIOD

\begin{tabular}{llll} 
& $-\ldots$ HIGH RAIN - - TSS - DURING CONSTRUCTION - - \\
LOCATION & MEAN & STD. ERR. & N \\
\hline UTR - ABOVE & 9.73 & 2.09 & 14 \\
TINKER CREEK & 12.12 & 2.35 & 13 \\
MCQUEEN BRANCH & 182.33 & 58.19 & 12 \\
UTR - MIDDLE & 15.27 & 3.32 & 7 \\
CROUCH BRANCH & 99.62 & 26.93 & 6 \\
UTR - BELOW & 17.69 & 3.96 & 7
\end{tabular}

$\begin{array}{ccc} & --- \text { HIGH RAIN - - TURBIDITY - DURING CONSTRUCTION - - } \\ \text { LOCATION } & \text { MEAN } & \text { STD. ERR. }\end{array}$

\begin{tabular}{llll}
\hline UTR - ABOVE & 4.34 & 0.66 & 14 \\
TINKER CREEK & 6.02 & 1.53 & 13 \\
MCQUEEN BRANCH & 236.13 & 62.53 & 12 \\
UTR - MIDDLE & 11.50 & 3.53 & 7 \\
CROUCH BRANCH & 187.83 & 47.98 & 6 \\
UTR - BELOW & 16.16 & 4.90 & 7
\end{tabular}

\begin{tabular}{lccc} 
LOCATION & - HIGH RAIN - PERCENT ASH - DURING CONSTRUCTION \\
& MEAN & STD ERR. & N \\
\hline UTR - ABOVE & 52.62 & 0.80 & 14 \\
TINKER CREEK & 51.23 & 1.62 & 13 \\
MCOUEEN BRANCH & 87.04 & 1.51 & 12 \\
UTR- MIDDLE & 56.04 & 5.23 & 7 \\
CROUCH BRANCH & 88.77 & 0.56 & 6 \\
UTR - BELOW & 63.24 & 3.20 & 7
\end{tabular}

- - - HIGH RAIN - - SPECIFIC CONDUCTANCE - - DURING CONSTRUCTION - LOCATION

UTR - ABOVE

TINKER CREEK

MCQUEEN BRANCH

UTR - MIDDLE

CROUCH BRANCH

UTR- BELOW
MEAN

17.38

32.91

66.80

65.30

108.27

25.32
STD. ERR.

N

$\begin{array}{ll}0.83 & 14 \\ 3.65 & 13 \\ 8.97 & 12 \\ 39.84 & 7 \\ 6.54 & 6 \\ 1.98 & 3\end{array}$


MEANS BY CONSTRUCTION PERIOD

LOCATION

- - - HIGH RAIN - - TSS - - AFTER CONSTRUCTION - MEAN TSS

STD. ERR.

N

\begin{tabular}{llll}
\hline UTR - ABOVE & 11.35 & 1.78 & 42 \\
TINKER CREEK & 15.74 & 1.99 & 42 \\
MCQUEEN BRANCH & 147.74 & 27.80 & 42 \\
UTR - MIDDLE & 21.66 & 4.38 & 21 \\
CROUCH BRANCH & 170.64 & 31.17 & 21 \\
UTR - BELOW & 43.74 & 12.88 & 21
\end{tabular}

LOCATION

- - - HIGH RAIN - - TURBIDITY - - AFTER CONSTRUCTION - MEAN

STD. ERR.

$N$

UTR - ABOVE

TINKER CREEK

MCQUEEN BRANCH

8.36

6.99

172.64

2.92

42

UTR - MIDDLE

24.23

1.23

42

CROUCH BRANCH

259.05

26.24

42

UTR - BELOW

50.82

5.95

21

44.20

21

16.80

21

-- HIGH RAIN - PERCENT ASH -
MEAN A

LOCATION

UTR - ABOVE

TINKER CREEK

MCQUEEN BRANCH

UTR - MIDDLE

CROUCH BRANCH

UTR - BELOW

57.01

53.31

85.87

66.99

87.80

71.54
AFTER STD ERR.

$\begin{array}{ll}1.18 & 42 \\ 1.11 & 42 \\ 0.79 & 42 \\ 2.41 & 21 \\ 1.62 & 21 \\ 2.53 & 21\end{array}$

- - - HIGH RAIN - - SPECIFIC CONDUCTANCE - - AFTER CONSTRUCTION - - LOCATION MEAN STD. ERR.

$N$

\begin{tabular}{llll}
\hline UTR - ABOVE & 17.86 & 0.43 & 42 \\
TINKER CREEK & 30.21 & 1.19 & 42 \\
MCQUEEN BRANCH & 57.68 & 7.81 & 42 \\
UTR - MIDDLE & 27.63 & 2.66 & 21 \\
CROUCH BRANCH & 82.92 & 6.44 & 21 \\
UTR - BELOW & 26.35 & 1.41 & 21
\end{tabular}




\section{AMPHIBIAN USE OF BREEDING SITES CREATED}

\section{AS EXPERIMENTAL MITIGATION FOR WETLAND LOSS}

Joseph H. K. Pechmann, Ruth A. Estes and David E. Scott

\section{INTRODUCTION}

Creation or restoration of wetlands is frequently required in the United States as mitigation for filling wetlands that are legally protected (Salvesen 1990). This mitigation is usually expected to be "in-kind" with respect to wetland type, as well as "on-site". However, there are few data on the extent to which wetlands constructed from uplands are ecological equivalents of those that they replaced (Kusler and Kentula 1990, Kentula et al. 1993). Postconstruction monitoring of these wetlands is rare. Even when monitoring occurs, it is often qualitative and short-term, and fails to consider faunal useage (Kusler and Kentula 1990, Kentula et al. 1993).

Constructed wetlands are typically planted with vegetation but not intentionally stocked with animals. The prevailing wisdom is that if vegetation and an appropriate hydroperiod are provided, fauna will colonize and use the site unless it is located many kilometers from other similar wetlands and has no surface water connections (Brooks 1990, Broome 1990, Erwin 1990, Hammer 1992).

Amphibians are an archtypical component of the fauna of freshwater wetlands, and large and diverge amphibian communities are often found in wetland habitats (Sharitz and Gibbons 1982, Moler and Franz 1988, Pechmann et al. 1989, Dodd 1992, Hammer 1992 ). There has been increased concern about declines and disappearances of amphibian populations worldwide, caused primarily by habitat destruction and modification (Wake and Morowitz 1990 ). It is important to ascertain the extent to which wetlands constructed to replace those lost to human activities maintain comparable populations of amphibians.

A Carolina bay wetland (Sharitz and Gibbons 1982, Ross 1987) called Sun Bay, located on the U.S. Department of Energy'g Savannah River Site in South Carolina, USA, was filled in 1983 for a construction project. Amphibian populations were monitored at sun Bay prior to and during construction. Four 
artificial ponds were built on the periphery of the construction site three montha before construction began, in an experimental attempt to mitigate the loss of amphibian habitat. Amphibian colonization and population dynamics were studied at these "refuge ponds" for eight and one half years to assess the success of the mitigation experiment. We here report the results. Comparisons are also made to studies conducted at a similar "reference wetland", Rainbow Bay, located $4 \mathrm{~km}$ from Sun Bay.

The wetland amphibian community

Most of the amphibian species that were found at sun Bay are primarily terrestrial (often fossorial), but migrate to ponds to breed and have an aquatic larval stage that lasts from two weeks to several months. Breeding seasons vary among species. Among the galamanders, Ambystoma opacum breeds in the autumn and Eurycea quadridigitata in the autumn and winter. Other Ambystoma and the chorus frogs Pseudacris breed in the winter in south Carolina. The spadefoot toad, Scaphiopus holbrooki, and the southern leopard frog, Rana utricularia, may breed after warm rains at any time of the year in this region. All other species that were found at sun Bay breed in the spring and summer. Although the salamander Notophthalmus viridescens breeds in the spring, mature efts and adults migrate to ponds during autumn and winter. Adult $N$. viridescens do not emigrate from ponds on the Savannah River site unless they dry, and then some may remain in the pond basin.

If a pond does not dry, larval Ambystoma talpoideum and Notophthalmus viridescens can delay the onset or completion of metamorphosis and become paedomorphic. That is, they can attain sexual maturity while remaining in the pond and retaining gills and sometimes other larval traits (Semlitsch and Gibbons 1985, Harris 1987, Reilly 1987). Paedomorphs of both species may later metamorphose.

The terrestrial home rangeg of pond-breeding amphibians are usually within a few hundred meters of their breeding pond (e.g., Jameson 1956, Kramer 1973, Williams 1973, Douglas and Monroe 1981, Semlitsch 1981, 1983b), although those of some species of Bufo (true toads) can extend up to $3 \mathrm{~km}$ away (Sinsch 
1990, 1992). The location of terrestrial home ranges has been studied for three salamander species that bred at Sun Bay. Ambystoma opacum migrated to home ranges an average of $193.7 \mathrm{~m}$ from a breeding pond in Indiana, range 0-450 m (Williams 1973). Ambystoma talpoideum adults were found to live 81-261 m from the pond, and juveniles $12-67 \mathrm{~m}$, in a study conducted at sun Bay and Rainbow Bay by semlitsch (1981). A single $A$. tigrinum was followed $162 \mathrm{~m}$ from Rainbow Bay by Semlitsch (1983b). Construction impacts extended at least 300 $\mathrm{m}$ from Sun Bay in all directions, and more than $500 \mathrm{~m}$ in most. Therefore, sun Bay amphibians lost most of their terrestrial habitat as well as their breeding site to construction.

Many amphibian species are philopatric, i.e., individuals usually use the same breeding site every year (e.g., Twitty 1959, Oldham 1966, Whitford and Vinegar 1966, Oldham 1967, Gill, 1978, Semlitsch 1981, Breden 1987, Semlitsch et al. 1988, Berven and Grudzien 1990, Reading et al. 1991, Scott 1994), and the same terrestrial home range (e.g., Haapanen 1970, Williams 1973, Breden 1987). Breeding adults are often philopatric to their natal pond (Breden 1987, Semlitsch et al. 1988, Berven and Grudzien 1990, Reading et al. 1991). Philopatry is ratsen Breden (1987) found that 738 of Bufo woodhousei fowleri used their natal pond for first reproduction, 248 used nearby ponds, and one used a pond $2 \mathrm{~km}$ away. Eightythree percent of adult $B$. $w$. fowleri bred in the same pond in consecutive years, while the other 178 relocated. Similar proportions of philopatry and dispersal have been reported for Bufo bufo, as well as a negative correlation between number relocating and distance between ponds (Reading et al. 1991). Rana sylvatica (Berven and Grudzien 1990) and Notophthalmus viridescens (Gill, 1978) were both found to be 100 faithful to breeding ponds as adults. Eighty-two percent of the R. sylvatica bred at their natal pond, while the other 188 dispersed to ponds an average of $1,169 \mathrm{~m}$ away (maximum 2,530 $\mathrm{m}$ ). Gill (1978) inferred that juvenile dispersal occurred in $N$. viridescens, occasionally to ponds many kilometers away. Marked adult Rana pipiens have been recaptured at wetlands as far as $5.2 \mathrm{~km}$ from their natal pond (Dole 
1971).

Philopatry can also vary with sex. Sinsch (1992) found that most male Bufo calamita were philopatric to their breeding pond, but that female $B$. calamita were not. Although adult philopatry to the natal pond is the rule for Ambystoma talpoideum (Semlitsch 1981, semlitsch et al. 1988) and A. opacum, (Scott 1994), individuals of both species that were marked at Rainbow Bay at metamorphosis have been found breeding at a pond $1 \mathrm{~km}$ distant (personal observations). Acoustic, magnetic, olfactory, and visual cues all appear to play a role in orientation and homing in amphibians (Twitty 1961, 01dham 1967, Landreth and Ferguson 1967, Taylor and Adler 1973, Grubb 1975, Hershey and Forester 1979, Phillips 1986, McGregor and Teska 1989, Sinsch 1990, Rodda and Phillips 1992).

Pond creation, pond 10ss, and amphibians

Amphibians are commonly observed to colonize ponds created intentionally or unintentionally by humans (Fog 1988, Schlupp et al. 1989, Brooks 1990, Laan and Verboom 1990, Adam and Lacki 1993, personal observations). Rates of colonization can be affected by distance from other ponds, characteristics of the intervening terrestrial habitat, dispersal capacity and site fidelity of the species, and size of source populations (Laan and Verboom 1990). In our study, new ponds were built a few months before the nearest natural wetland and much of the surrounding terrestrial amphibian habitat were eliminated. It was clear that significant direct amphibian mortality would result from bulldozing and other construction activities, reducing the number of potential colonizers. A central question was whether surviving individuals would breed at the new refuge ponds or return to sun Bay, even though it had been drained and filled and the surrounding vegetation and topography had been drastically altered.

There have been several reports of amphibians returning to former breeding sites even after they had been filled: Anderson (1954) for Gastrophryne carolinensis, Heusser (1960) for Bufo bufo, Shoop (personal observation, cited in Shoop and Doty 1972) for Ambystoma talpoideum, Uzzell 
(personal communication, cited in Shoop and Doty 1972) for A. laterale, and Frazer (1973) for unspecified frogs and toads. The subsequent fate of these populations was not reported except by Frazer. He noted that a few made their way to an undrained part of the breeding canal, where they became established after being assisted over an embankment.

Schlupp et al. (1989) successfully relocated Bufo bufo to a newlyconstructed breeding site by capturing them on their way to another pond, placing them the new pond, and keeping them there with a fence throughout the breeding season. Within three years, most of the Bufo bred in the new pond of their own accord. Cook and Pinnock (1987), Cook (1989), and Matthews et al. (1991), reported success at establishing amphibian populations in newly-built ponds by stocking eggs, larvae, or adults, mostly from nearby sites threatened by development.

We chose not to stock our ponds, because amphibians and other fauna are not usually stocked in constructed wetlands, and we wanted to study the effectiveness of typical procedures. The philosophy of our study was to simply create ponds, let them fill with rainwater, and allow colonization and succession to take their course. We did not attempt to mitigate for any other functions of the lost wetland other than its role as an amphibian breeding site.

\section{METHOD8 \\ Study site}

The study was conducted in Aiken County, South Carolina, USA on the U.S. Department of Energy's $780-\mathrm{km}^{2}$ Savannah River Site. Sun Bay was a Carolina bay, one of thousands of elliptical depressions of unresolved geological origin common to the southeastern U.S. Atlantic Coastal Plain (Sharitz and Gibbons 1982, Ross 1987). Sun Bay was approximately 1 ha in area, had a maximum depth of about $1 \mathrm{~m}$, and had no surface connection to other bodies of water. It was a temporary pond that usually filled in the winter and dried in the spring or summer. Filling and drying dates varied widely from year to year, depending on temperature and rainfall (Semlitsch 1983a, Semlitsch 1987, 
Caldwell 1987, Pechmann et al. 1989). In 1978, before our study began, a ditch was dug that partially drained the bay for pre-construction surveys. The ditch reduced water volume by about $75 \%$, decreased depth (maximum was 0.35 m during our study), and caused the pond to dry earlier each year (Semlitsch 1983a, 1987, Caldwell 1987, Pechmann et al. 1989). Thus, our pre-construction studies were not pre-disturbance.

In 1979, the center part of sun Bay contained herbaceous vegetation, primarily Panicum sp. (panic grass), Juncus spp. (rushes), Eleocharis spp. (spike rushes), Polygonum sp. (knotweed), and Viola lanceolata (lance-leaved violet). Cephalanthus occidentalus (buttonbush) grew primarily around the outer edge of the herbaceous area, but also in the middle. A forested zone of Liquidamber styraciflua (sweetgum) occupied the periphery of the bay. The composition and extent of these vegetation zones probably varied over time in response to variation in hydroperiod and other factors, as has been observed in other Carolina bays such as Rainbow Bay (personal observations). Approximately 408 of the bay's vegetation was cleared with a bulldozer in 1978 for pre-construction seismic surveys and for the ditch. Weedy pioneer species invaded these bare areas.

Terrestrial habitats within a $1 \mathrm{~km}$ radius of Sun Bay consisted primarily of deep, well-drained sandy soils planted with Pinus taeda (loblolly pine) and P. elliottii (slash pine). These pine stands were of various ages, the oldest having been planted in the early 1950 s on abandoned agricultural fields, and the youngest in 1975 following a clear-cut. Two of the refuge ponds, $B$ and $D$ (Fig. III-1), were located in $P$. elliottii plantations that also contained scattered $P$. taeda. The area around Pond $D$ had a well-developed mixed species understory and shrub layer, and only a thin litter layer, perhaps because of prescribed burning. Pond $B$ was surrounded by disturbed soil in which the trees grew poorly. The understory around Pond B contained only pine.

Other plant communities found within $1 \mathrm{~km}$ of sun bay included Pinus palustris (longleaf pine) stands, a stand of Quercus laevis (turkey oak), mixed upland hardwoods, and bottomland hardwoods. Refuge Pond C (Fig. III-1) 
was located in a longleaf pine stand having a hardwood midstory and a thick litter layer. Pond A (Fig. III-1) was on a slope, and surrounded by a forest dominated by mixed hardwoods on the south and southwest and $p$. taeda elsewhere. The forest on all sides of Pond A was well-stratified, having well-developed hardwood shrub and ground vegetation layers compared to the other refuge pond sites.

Human structures within $1 \mathrm{~km}$ of sun Bay before the new construction included part of an industrial site, a borrow pit, two paved roads, two dirt roads, and a power line (Fig. III-1). These structures were surrounded by mowed grass or herbaceous weeds. Numerous paths were cleared with a bulldozer through the vegetation around Sun Bay during pre-construction surveys that began in 1978 .

Two small streams, McQueen Branch and Crouch Branch, are each located within $1 \mathrm{~km}$ of the site of Sun Bay (Fig. III-1). These streams oupport a different suite of amphibian populations than did the bay, including Amphiuma means (two-toed amphiuma), Desmognathus auriculatus (southern dusky salamander), Pseudotriton ruber (red salamander), $P$. montanus (mud salamander), and Eurycea cirrigera (two-lined salamander). The only other aquatic habitats within $1 \mathrm{~km}$ of sun Bay were roadside ditches and small, temporary puddles, but these habitats are used extensively as breeding sites by species such as Scaphiopus holbrooki (eastern spadefoot toad), Gastrophryne carolinensis (eastern narrow-mouthed toad), and Hyla squirella (gquirrel treefrog). There is a farm pond $1.2 \mathrm{~km}$ east-southeast of the former bay, and four Carolina bays each approximately $2 \mathrm{~km}$ away, two in a northeast and two in a south-southeast direction. Thus, individuals from sun Bay were not the only potential source of colonists for the refuge ponds.

\section{The construction project}

The U.S. Department of Energy Defense Waste Processing Facility and associated structures were built on the site of sun Bay and the surrounding area. Clearing and grading began in september 1983, and encompassed the tract between Savannah River Site Road F on the north, site Road 4 on the northwest, 
the powerline on the west, and the $H$ area industrial site on the south, to beyond McQueen Branch on the southeast (Fig. III-1). Sun Bay was filled with soil in October 1983 while the bay was dry. Standing water collected in a low area on the NW side of the former wetland after rains in mid-November. The water remained until 21 December 1983, when a ditch was dug that permanently drained the site. The site of the former wetland became a parking lot for the facility.

Three sedimentation ponds were built near the perimeter of the cleared and graded area, one on Crouch Branch and two on Mcqueen Branch. These ponds, completed in June 1984, were designed to mitigate the impacts of erosion from the construction site on these streams. We did not monitor amphibian populations at these sedimentation ponds, but they have probably provided suitable breeding sites for some species.

clearing and grading for an associated facility, z-area, built north of Site Road F, began in the summer of 1985 (Fig. III-1). The power line located on the west side of the construction site was moved closer to site Road 4 in the autumn of 1985, disturbing additional terrestrial habitat around one of the refuge ponds (Fig. III-1). Exterior construction work on all facilities was completed by $\mathrm{FY}-1988$, but interior work and testing were still ongoing in FY-1993.

\section{Refuge pond design}

The four refuge ponds (A, B, C, and D) were completed on 20 June 1983 (Fig. III-1). They were built between $300 \mathrm{~m}$ and $600 \mathrm{~m}$ from Sun Bay, which was as close as permitted by construction plans (including $\mathrm{z}$-Area). When possible, sites for ponds were chosen where puddles of water tended to collect naturally. The disturbed soil at the site of Pond B suggested that it may have been an old soil borrow area. A paved two-lane road lies between Sun Bay and three of the refuge ponds and a powerline right-of-way containing a dirt road lies between the bay and the fourth pond (Fig. III-1). The effect of these barriers on amphibian movements is unknown, but it was probably no greater than that of the widespread clearing and grading from construction 
activities.

Each pond is circular, approximately $16 \mathrm{~m}$ in diameter, and has a maximum depth in the middle of approximately $1 \mathrm{~m}$. Each is $200 \mathrm{~m}^{2}$ in area, which is a total of $800 \mathrm{~m}^{2}$ for 4 ponds. The wetland they replaced was approximately $10,000 \mathrm{~m}^{2}$, so our study should be viewed as an experimental "pilot project", not as mitigation per se. Plywood boards were placed around each pond to provide shelter for amphibians until natural vegetation could become established.

Ponds were originally lined with $20-25 \mathrm{~cm}$ of hard-packed clay so that they would collect and hold rainwater. Most Carolina bays are underlaid by an impervious clay lens, and usually receive no water input other than rain (Bryant and MCCracken 1964, Sharitz and Gibbons 1982, Schalles and Shure 1989, Lide 1991). Rainfall was monitored daily with a rain gauge. The water level of each pond and of Rainbow Bay was monitored weekly with a staff gauge located in the middle.

Refuge pond water retention was poor during the first year after they were built, in spite of high rainfall. To rectify this problem fish-grade plastic (CPE) pond liners were installed on 19 November 1984. Because the plastic liners initially provided an inert substrate, terrestrial leaf litter was added to the ponds during February and March 1985. These leaves supplied cover, nutrients, and organic matter for biota.

After installation of the plastic liners the refuge ponds became permanent ponds. Refuge Ponds $A$ and $B$ were each pumped to one-third of their depth from 28-29 September 1987 (Pond A from $63 \mathrm{~cm}$ to $22 \mathrm{~cm}$, Pond B from $89 \mathrm{~cm}$ to $29 \mathrm{~cm})$. Both ponds were dried completely by pumping and hand bailing from 19 October 1987 to 22 October 1987, then allowed to refill with rain beginning 27 October 1987. These manipulations were designed to simulate the annual drying characteristic of Rainbow Bay and the former sun Bay.

At the request of the Department of Energy, Refuge Pond $\mathrm{C}$ was dismantled on 7 June 1985 to accommodate expansion of the planned $\mathrm{z}$-Area. 


\section{Sampling techniques}

We monitored amphibian populations at the refuge ponds using terrestrial drift fences with pitfall traps (Gibbons and Semlitsch 1982). These were installed 20-21 June 1983 and deactivated 9 December 1991. Fences were of 50$\mathrm{cm}$-high aluminum flashing buried $10 \mathrm{~cm}$ in the ground, and completely encircled each pond. Pitfall trapg were 40 liter buckets buried flush against the fence on each side every $10 \mathrm{~m}$. Traps were checked daily, and animals released on the opposite side of the fence, the presumed direction of movement.

Most amphibians captured were marked by toe-clipping. A combination of individual marks, year-pond cohort marks, and general group marks was used. Our sampling techniques provided a census of the numbers of adults that entered each pond to breed each year, as well as the numbers of newlymetamorphosed juveniles that emigrated to surrounding terrestrial habitats.

The capture efficiency of drift fences with pitfall traps differs among species (Gibbons and Semlitsch 1982, Dodd 1991, personal observations). Practically all individuals of some species are captured, for example, ambystomatid salamanders and bufonid toads. Other amphibians such as treefrogs and ranid frogs frequently jump or climb over the fence and out of the traps. Juveniles in these groups are captured more effectively than adults, as the juveniles are less adept at trespass. Analyses of population trends over time make the assumption that there is no trend in the sampling efficiency of the drift fences, which is reasonable if the fences and traps are maintained well. Comparisons among sites assume that the capture efficiency of drift fences and pitfall traps of the same design does not differ among sites, which is most defensible for fences that completely encircle ponds.

A drift fence with pitfall traps was used to monitor amphibian migrations at sun Bay prior to construction from 8 February 1979-24 June 1982, after which sampling was discontinued at the request of the Department of Energy. The fence and traps were of the same design used at the refuge ponds, and most amphibians captured at Sun Bay were also marked by toe-clipping. 
These data are summarized only briefly here for comparative purposes. Further details are provided by SREL (1980), Vitt (1981), Vitt et al. (1982), Semlitsch (1983a, 1987), Caldwell (1987), and Pechmann et al. (1989).

Drift fences with pitfall traps also were used to monitor amphibian breeding migrations to the site of the former sun Bay during construction. Because of the extensive construction activities, these fences were employed intermittently and covered only a small part of the perimeter of the former wetland. Four-and-one-half-liter pitfall traps were used at these fences instead of the 40-liter traps used elsewhere to facilitate frequent installation and removal. These small traps were not as effective as the larger traps.

One 50-m drift fence located on the northwest gide of the former bay was used for sampling from 13 December 1983 to 11 May 1984. Two temporary 50-m fences were employed 17 December 1984-3 July 1985 and 21 November 1985-29 August 1986: one on the northwest side and the other on the northeast. A single fence on the northeast side was used for sampling from 29 August 1986 to 16 september 1987. Monitoring of the Sun Bay site was discontinued after this date because captures of amphibians had dwindled to near zero. Sampling differences cloud among-year comparisons of amphibian populations at sun Bay during construction, as well as comparisons to the refuge ponds, but were unavoidable due to the construction activities.

In addition to the drift fences, the standing water at the edge of the former Sun Bay was sampled with minnow traps from 12 December 1983 until 21 December 1983, after which the site was completely drained by a ditch. We then sampled the drainage ditch with the minnow traps until 31 March 1984. Each refuge pond was sampled with minnow traps from 10 January 1987 to 15 April 1987 in order to determine if paedomorphic salamanders were present. All minnow traps were checked daily and all captured animals released immediately. Adult amphibians and salamander larvae captured in minnow traps were marked by toe-clipping. Each refuge pond was surveyed qualitatively with dip nets and a seine on 17 April 1991 for the presence of adult Notophthalmus 
viridescens.

\section{RESULTS}

Sun Bay

Four to six species of salamanders and seven to 15 species of frogs and toads probably bred at Sun Bay prior to construction (Table III-1). Breeding population sizes varied considerably during the pre-construction survey (19791982). No juvenile recruitment was observed during this period for some species, and for the others recruitment was low and sporadic (Table III-1). Observations indicated that this resulted in part from the fact that the sun Bay usually dried before many larvae could reach the minimum size for metamorphosis. Ambystoma talpoideum and Notophthalmus viridescens were the most commonly captured salamanders before construction, and Bufo terrestris, Gastrophryne carolinensis, Pseudacris crucifer, P. ornata, and Scaphiopus holbrooki the most commonly captured frogs and toads.

Some species continued breeding migrations to the site of Sun Bay for a period of time after it was filled with soil in 1983 (Table III-2). By the 1987 breeding seasons, however, only one breeding adult was captured at sun Bay, a female Rana utricularia.

The refuge pond environment

The hydrologic cycles of the refuge ponds did not match that of the undisturbed reference wetland, Rainbow Bay (Fig. III-2). Rainbow Bay usually filled in the winter and dried in the spring or summer, depending upon rainfall, as had sun Bay. The refuge ponds filled and dried more frequently during the first 17 months after they were built than did Rainbow Bay (Fig III-2). Only ponds $A$ and $B$ held water for more than a few weeks at a time during this period, as they were built on sites where water tended to collect naturally. The refuge ponds became permanent ponds after the pond liners were installed (Fig. III-2). Ponds $A$ and $B$ remained dry for less than a week after the water was pumped out of them in 1987 (Fig. III-2). This was a shorter dry period than exhibited by Rainbow Bay during any year of the study.

Grasses and forbs initially colonized the bare soil that surrounded each 
pond after their construction. These were gradually replaced by volunteer Pinus taeda at Pond $A$ and $P$, elliottii at ponds $B$ and $D$. There were annual blooms of filamentous green algae in the ponds. An emergent sedge (Scirpus cyperinus) became established in the shallow water along the shores. Aquatic insects, including large predaceous odonata nymphs, became common in the ponds. Crows, sandpipers, and other birds often fed in the water around the edges of the ponds.

\section{Frogs and Toads}

The frogs and toads Bufo terrestris, Gastrophryne carolinensis, Pseudacris crucifer, and Rana utricularia colonized the refuge ponds within a year, and breeding populations of these species were present at the ponds during every year of the study (Fig. III-3, Table III-3). Smaller numbers of adults of 9 other species of anurans were captured during their breeding seasons in one or more years at the refuge ponds (Fig. III-3, Table III-3). Numbers must be judged keeping in mind that sampling efficiencies were low for adult Acris, Rana and Hyla. As an extreme example, no adult $H$. gratiosa were ever captured in the refuge pond pitfall traps even though juveniles were produced at all three remaining ponds every year beginning in 1985 (Table III3). More adult Scaphiopus holbrooki, B. quercicus, H. femoralis, H. chrysoscelis, and $R$. clamitans were captured in the firgt part of the study than the latter part, and the reverse was true for A. gryllus, but sample sizes were too small to draw any conclusions about trends. More individuals of all frog and toad species were caught at the refuge ponds than at sun Bay each year that both locations were monitored, except 1984 for $P$. ornata, but sampling efficiency was greater at the refuge ponds (Fig. III-3, Tables III-2 and III-3). Only four B. terrestris, one $G$. carolinensis, and two of the $R$. utricularia captured at the refuge ponds had probably been marked at sun Bay during pre-construction surveys.

No juveniles metamorphosed and emigrated from the refuge ponds in 1983, and only 7 juvenile Hyla chrysoscelis and 1 juvenile Hyla femoralis were observed in 1984 (Fig. III-3, Table III-3). Observations indicated that the 
low juvenile recruitment during these first two yearg was due in part to the fact that the ponds dried frequently, killing the tadpoles that were present. Substantial production of frog and toad juveniles began in 1985 following installation of the pond liners. Juveniles of ten species of frogs and toads metamorphosed and emigrated from the refuge ponds during the study (Fig. III3, Table III-3). The numbers of juveniles produced varied considerably among species, ponds, and years. Pseudacris crucifer and Rana utricularia produced the most frog and toad juveniles at the refuge ponds. The largest cohort of both species was produced in 1985, the first year that the ponds filled with water for an extended period (Figs. III-2 and III-3, Table III-3). The next largest cohort of both species came in 1988, primarily from ponds $A$ and $B$ which had been pumped dry in the autumn of 1987. Nearly all $R$. utricularia juveniles also came from Ponds $A$ and $B$ in other years, whereas Pond $D$ produced the most $P$. crucifer juveniles overall. P. crucifer had been one of the three dominant species at sun Bay in terms of juvenile recruitment during preconstruction studies, but no juvenile recruitment of $R$. utricularia was observed there from 1979-1982 (Table III-1).

Relatively large numbers of juvenile Acris gryllus, Bufo terrestris, and Hyla gratiosa also metamorphosed and emigrated from the refuge ponds (Fig. III-3, Table III-3). The largest number of all three species came from Pond $D$, but nearly as many $H$. gratiosa came from Pond $B$. No juvenile recruitment of A. gryllus was observed at the ponds until 1987, the same year that adults of this species were first captured. After that a cohort of Acris was produced every year, the largest during the last year of the study. The only cohort of Rana clamitans was produced at Pond $A$ during the last year of the study. In contrast to $A$. gryllus and $R$. clamitans, juvenile recruitment of Hyla chrysoscelis at the refuge ponds occurred primarily in the first part of the study. Eighty-six percent of the juvenile $H$. chrysoscelis were collected in 1985, primarily from ponds $A$ and B. No juvenile recruitment of Acris gryllus, Hyla chrysoscelis, or H. gratiosa was observed at Sun Bay from 19791982 . 
Only a few Rana catesbeiana and Pseudacris ornata juveniles and a single Hyla femoralis juvenile were produced at the refuge ponds (Fig. III-3, Table III-3). Most of the $P$. ornata came from Pond $B$ in the year after it was pumped dry. Pseudacris ornata had been one of the dominant frogs and toads at Sun Bay in terms of juvenile recruitment, but no $R$. catesbeiana or $H$. femoralis recruitment was observed there during pre-construction studies (Table III-1). No juvenile Gastrophryne carolinensis or Scaphiopus holbrooki were produced at the refuge ponds, despite the fact that adults of these species were frequently captured there. Scaphiopus holbrooki was the only frog or toad that produced juveniles at sun Bay during pre-construction studies but did not produce any at the refuge ponds.

\section{Salamanders}

More adult Ambystoma talpoideum and Notophthalmus viridescens were caught at Sun Bay than at the refuge ponds during FY-1984, despite the construction and the less efficient sampling at sun Bay (Table III-2, Fig. III-4). Few salamanders were captured at either location in FY-1985, a drought year. More adult $A$. talpoideum and $N$. viridescens were caught at the refuge ponds than at Sun Bay during the next two years, which were the last that Sun Bay was sampled, although numbers are not directly comparable because sampling effort was greater at the refuge ponds (Table III-2, Fig. III-4). The number of these two species that entered the refuge ponds during their FY1986 breeding seasons was higher than in previous years, especially for Ambystoma talpoideum at Pond D (Fig. III-4). These higher numbers of immigrating adult salamanders generally persisted from FY-1987 to FY-1989, and numbers increased sharply again in FY-1990.

Three of the $19 \mathrm{~N}$. viridescens and 46 of the $99 \mathrm{~A}$. talpoideum adults that entered the refuge ponds during the first 4 years of the study had been marked previously at sun Bay. Ambystome talpoideum and $N$. viridescens were the most common salamanders at Sun Bay prior to construction (Table III-1). Three other species of salamanders were collected at the sun Bay drift fence prior to construction: A. opacum, A. tigrinum, and Eurycea quadridigitata 
(Table III-1). Only a few individuals of these species were captured at sun Bay or the refuge ponds after construction began (Tableg III-1 and III-2; Fig. III-4). None of those captured at the refuge ponds had been marked previously at Sun Bay.

Juvenile salamanders were not produced at the refuge ponds until FY1986, the first year of notable colonization by adults (Fig. III-4). Hundreds of Ambystoma talpoideum and Notophthalmus viridescens metamorphosed and emigrated from the refuge ponds from FY-1986 to FY-1991 (Fig. III-4). These are the only two salamander species for which juvenile recruitment has been observed at the refuge ponds, however. Each of the two specles became established primarily at two of the three remaining refuge ponds. Only $10 \mathrm{~A}$. talpoideum have metamorphosed and emigrated from Pond $A$, and only two $N$. viridescens from Pond $B$. Pond $D$ is the only one at which both species have produced cohorts during the last four years of the study.

By the end of the study, most of the adult Ambystoma talpoideum migrating to the refuge ponds to breed had been born at them. Out of a sample of 228 A. talpoideum marked at metamorphosis that subsequently returned, 958 returned to their home refuge pond and 58 returned to a different refuge pond. All but one of the movements among ponds were between ponds $B$ and $D$, the two ponds that most of the metamorphosed $A$. talpoideum have come from. The presence of paedomorphic Ambystoma talpoideum and Notophthalmus viridescens at the refuge ponds was established by their capture in minnow traps during FY-1987 (Table III-3, part II-A). Mature male paedomorphs of each species can be recognized externally during the breeding season by the appearance of their cloaca and the presence of gills. Mature female paedomorphs cannot always be differentiated from overwintering larvae of either sex without dissection. Yolked ova were visible through the venter of some of the gilled $N$. viridescens caught in the minnow traps, indicating that they were mature paedomorphic females. Yolked ova were not observed through the venter of any of the gilled A. talpoideum, but most may have bred before the minnow traps were deployed. One or two gilled males and females of each 
species were dissected to confirm that they were sexually mature. Ambystoma talpoideum paedomorphs were found primarily in ponds $B$ and $D$, and $N$. viridescens paedomorphs were caught only in ponds $A$ and $D$.

Some of the known paedomorphic male and suspected paedomorphic female Ambystoma talpoideum captured in the minnow traps in FY-1987 metamorphosed and emigrated from the refuge ponds during March and April of that year (Table III-3, part II-A). One of these females was dissected and confirmed to be a former paedomorph. A subset of these males and females returned to the refuge ponds to breed as metamorphs in subsequent years.

None of the paedomorphic Notophthalmus viridescens captured in the minnow traps were ever captured as metamorphs at the drift fence (Table III-3, part II-A). Some of the $N$. viridescens captured with netg in April 1991 were adults whose gills were reduced to small nubs, indicating that some paedomorphic individuals metamorphosed but remained in the ponds. Notophthalmus viridescens were captured only at refuge ponds $A$ and $D$ in the April 1991 survey.

Ambystoma talpoideum metamorphosed and emigrated from the refuge ponds throughout the year, although primarily in the spring and autumn. Sex and maturity could not be judged externally in most individuals that metamorphosed. A sample of 23 newly-metamorphosed individuals collected in March and April of 1987 and 1991 was dissected and found to consist of a mixture of juveniles and former paedomorphs of both sexes. There was often a natural break in the migration of newly-metamorphosed $A$. talpoideum from the refuge ponds around the middle of May, just before $A$. talpoideum juveniles begin to metamorphose and emigrate from temporary ponds without paedomorphs such as Rainbow Bay. Individuals that emigrated from the refuge ponds from 20 May-31 December were therefore judged to consist primarily of juvenile youngof-the-year. Ambystoma talpoideum that metamorphosed and emigrated from 1 January to 19 May were considered to be a mix of overwintering juveniles and former paedomorphs. These individuals were added to the juveniles from the previous calendar year, but as a separate category (Fig. III-4). 


\section{DISCUSSION}

During FY-1984, the first complete year of our study and of DWPF construction, salamanders continued to return to sun Bay despite the ongoing construction. The few adult salamanders that entered the refuge ponds during FY-1984 left within a few days (Pechmann et al. 1984). During FY-1985 only one adult salamander was caught at the former site of sun Bay, and three at the refuge ponds. Lack of opportunities to migrate due to low rainfall during FY-1985 probably contributed to the low number of captures both at these sites and at the Rainbow Bay control site (Pechmann et al. 1985, Pechmann and Semlitsch 1986).

Although much of FY-1986 was also comparatively dry, heavy rains during late November and early December provided salamanders with adequate opportunities to migrate to breeding sites. Record numbers of three salamander species entered Rainbow Bay during FY-1986. Large numbers of two of these species, Ambystoma talpoideum and Notophthalmus viridescens, also entered the refuge ponds. Ambystoma talpoideum and $N$. viridescens normally return to breed at the site where they were hatched (Semlitsch 1981, D. E. Gill, personal communication). Apparently some individuals of these species responded to the elimination of sun Bay and other disturbances from construction by migrating to the refuge ponds rather than returning to sun Bay. Ambystoma talpoideum populations philopatric to the individual refuge ponds have now been established from the sun Bay populations.

Preliminary results indicate that the refuge ponds provide adequate salamander breeding habitat. Both $A$. talpoideum and $N$. viridescens have bred in the refuge ponds since FY-1986, and at least some of their larvae successfully developed through metamorphosis each year. The presence of paedomorphic adults provides additional evidence that the refuge ponds provide favorable habitat for salamanders (but see Whiteman 1994).

Several species of frogs and toads had colonized the refuge ponds during the first two years of the study (Pechmann et al. 1984, Pechmann et al. 1985). These anuran species may be less philopatric than the salamander species that 
formerly bred at Sun Bay (personal observations), although differences in speed of travel, response to construction, and other factors might also have contributed to their more rapid colonization.

Lack of seasonal pond drying at the refuge ponds may have reduced or eliminated colonization and juvenile production by some species, and promoted it in others. Pseudacris ornata seem to prefer to breed in newly-filled temporary ponds, and therefore may not have colonized the refuge ponds extensively. Lack of pond drying may have hindered their reproductive success as well as that of Scaphiopus holbrooki and Gastrophyne carolinensis, which also usually breed in newly-filled ponds. The largest total numbers of frog and toad juveniles produced were in FY-1985, the year that the liners were installed and the ponds began to hold water for more than short periods, and FY-1988, the year that two of them were artificially dried. Drying reduces the numbers of insect and salamander predators, and may increase nutrients by allowing soil oxidation. On the other hand, Acris gryllus prefers more permanent ponds and became more common at the refuge ponds than at Rainbow Bay, which usually dries seasonally.

Refuge ponds should have a hydrologic cycle similar to that of the original breeding site for maximal success. Ponds that hold water for a shorter or longer period of time each year on the average, or dry more or less frequently than the breeding aite they replaced, might support a different amphibian community and a lower density and diversity of amphibians (scott et al. 1986). Our experience with the DWPF refuge ponds has demonstrated that building a perched water table system such as that found in Carolina bays (Schalles 1979) is not an easy task. The original pond design did not hold water well enough, but adding pond liners turned them into permanent ponds. Future mitigation efforts should include attempts to mimic more carefully the natural wetland system through construction of larger ponds, alteration of pond depth and configuration, and experimentation with other types of drainage mechanisms. Such approaches must be coupled with continued surveillance of amphibian colonization patterns, as well as the physical and hydrologic 
aspects of the ponds, in order to evaluate the ouccess of this type of mitigation.

Numbers and diversity of amphibians at the Rainbow Bay control site have generally exceeded those at the refuge ponds. Juvenile production at Rainbow Bay in many years has been reduced by early pond drying due to drought (Pechmann et al. 1989, 1991), whereas the refuge ponds have not dried since pond liners were installed in FY-1985.

The refuge pond concept appears to have much potential for mitigating the loss or degradation of amphibian breeding habitat on the sRs as well as at other locations. However, resultg to date indicate that they may provide only partial mitigation. Several species of amphibians that were formerly common at Sun Bay have not yet successfully colonized the refuge ponds, notably Ambystoma opacum and Ambystoma tigrinum. Breeding population sizes at sun Bay of these two unsuccessful salamander species were less than 100, compared to over 1000 for the successful salamanders, so we think that the probability that surviving individuals would find and use the refuge ponds was simply much lower. A few Ambystoma tigrinum were caught at the refuge ponds, but we never got a male and a female in the same pond at the same time. If mitigation was being undertaken primarily for the benefit of ' rare or endangered species, our results suggest that there is no guarantee that they would become established in the new habitat.

Finally, community structure diverged among the three replicate pondg, probably in part due to chance historical effectg during initial colonization. As near as we can tell, these differences among ponds resulted from the stochastic effects of initial colonization. More Notophthalmus than A. talpoideum bred in Pond A initially, while the opposite happened in Pond B. Apparently a sufficient number of both species colonized Pond D for both to become established. Thus, our results suggest that chance alone may result in the establishment of a different amphibian community in artificial wetlands than that which was found in the one they replaced.

Building replacement wetlands as mitigation for the elimination or 
degradation of natural wetlands is required in many areas under certain conditions. However, there are very little data to indicate whether or not this is a useful exercise. Studies such as ours will be useful to the Department of Energy as well as other groups in planning how to better manage wetland ecosystems and minimize the impacts of man upon them. 


\section{IITERATURE CITED}

Adam, M. D. , and M. J. Lacki. 1993. Factors affecting amphibian use of road-rut ponds in Daniel Boone National Forest. Transactions of the Kentucky Academy of Science 54:13-16.

Anderson, P. K. 1954. Studies in the ecology of the narrow-mouthed toad, Microhyla carolinensis carolinensis. Tulane studies in Zoology $2: 15-46$

Berven, K.A., and T.A. Grudzien. 1990. Dispersal in the wood frog (Rana sylvatica): implications for genetic population structure. Evolution 44:2047-2056.

Breden, F. 1987. The effect of post-metamorphic dispersal on the population genetic structure of Fowler's toad, Bufo woodhousei fowleri. Copeia $1987: 386-395$.

Brooks, R.P. 1990. Wetland and waterbody restoration and creation associated with mining. Pages 529-548 in J.A. Kusler and M. E. Kentula, editors. Wetlands creation and restoration: the status of the science. Island Pregs, Washington, D.C., USA.

Broome, S.W. 1990. Creation and restoration of tidal wetlands of the southeastern United States. Pages 37-72 in J.A. Rusler and M. E. Kentula, editors. Wetlands creation and restoration: the status of the science. Island Press, Washington, D.C., USA.

Bryant, J.P., and R.J. McCracken. 1964. Properties of soils and sediments of the Carolina bays. Journal of the Elisha Mitchell Scientific society. $80: 166$. 
Caldwell, J. P. 1987. Demography and life history of two species of chorus frogs (Anura: Hylidae) in South Carolina. Copeia $1987: 114-127$

cook, R. P. 1989. And the voice of the grey tree frog was heard again in the land. Park science $9(3): 6-7$.

Cook, R. P., and C. A. Pinnock. 1987. Recreating a herpetofaunal community at Gateway National Recreation Area, New York. 151-154 in L. W. Adams and D. L. Leedy. Integrating man and nature in the metropolitan environment. Proceedings of the National Symposium on Urban Wildlife, Chevy Chase, Maryland, 4-7 November 1986. National Institute for Urban wildlife, Columbia, Maryland, USA.

Dodd, C. K., Jr. 1991. Drift fence-associated sampling bias of amphibians at a Florida Sandhills temporary pond. Journal of Herpetology $25: 296-301$

Dodd, C.R.,Jr. 1992. Biological diversity of a temporary pond herpetofauna in north Florida sandhills. Biodiversity and Conservation $1: 125-142$.

Dole, J. W. 1971. Dispersal of recently metamorphosed leopard frogs, Rana pipiens. Copeia 1971:221-228.

Douglas, M.E., and B.L. Monroe, Jr. 1981. A comparative study of topographical orientation in Ambystoma (Amphibia: Caudata). Copeia $1981: 460-463$

Erwin, K.L. 1990. Freshwater marsh creation and restoration in the southeast. 
Erwin, K.L. 1990. Freshwater

h creation and restoration in the southeast. Pages 233-2, n J.A. Kusler and M. E. Kentula, editors. Wetlands crition and restoration: the gtatus of the science, Island Press, Washington, D.C., USA.

Fog, K. 1988. Pond restorations on Bornholm. Memoranda Societas Fauna Flora Fennica $64: 143-145$.

Frazer, J. D. 1973. Amphibians. Wykeham Publications, London, England.Gill, D. E. 1978. The metapopulation ecology of the red-spotted newt, Notophthalmus viridescens (Rafinesque). Ecological Monographs $48: 145-166$.

Gibbons, J.W., and R.D. Semlitsch. 1982. Terrestrial drift fences with pitfall traps: an effective technique for quantitative sampling of animal populations. Brimleyana $7: 1-16$.

Gill, D. E. 1978. The metapopulation ecology of the red-spotted newt, Notophthalmus viridescens (Rafinesque). Ecological Monographs 48:145-166.

Grubb, J. C. 1975. Olfactory orientation in southern leopard frogs, Rana utricularia. Herpetologica 31:219-221.

Haapanen, A. 1970. Site tenacity of the common frog (Rana temporaria L.) and the moor frog ( $R$. arvalis Nilss.). Annales Zoologici Fennici $7: 61-66$.

Hammer, D.A. 1992. Creating freshwater wetlands. Lewis Publishers, Boca Raton, Florida, USA. 
Harris, R. N. 1987. Density-dependent paedomorphosis in the salamander Notophthalmus viridescens dorsalis. Ecology 68:705-712.

Hershey, J.I., and D.C. Forester. 1979. Sensory orientation in Notophthalmus v. viridescens (Amphibia: Salamandridae). Canadian Journal of Zoology $58: 266-276$.

Heusser, H. 1960. Über die beziehungen der erdkröte (Bufo bufo L.) zu ihrem laichplatz II. Behaviour 16:93-109.

Jameson, D.I. 1956. Growth, dispersal and survival of the Pacific tree frog. Copeia 1956:25-29.

Kentula, M. E., R. P. Brooks, S. E. Gwin, C. C. Holland, A. D. Sherman, and J. c. Sifneos. 1993. An approach to improved decision-making in wetland restoration and creation. Lewis Publishers, Boca Raton, Florida.

Kramer, D.C. 1973. Movements of western chorus frogs pseudacris triseriata triseriata tagged with $\mathrm{Co}^{\infty}$. Journal of Herpetology 7:231235.

Kusler, J. A., and M. E. Kentula, eds. 1990. Wetlands creation and restoration: the status of the science. Island Press, Washington, D.C., USA.

Laan, R. , and B. Verboom. 1990. Effects of pool size and isolation on amphibian communities. Biological Conservation 54:251-262.

Landreth, H.F., and D.E. Ferguson. 1967. Newt orientation by sun-compass. Nature 215:516-518. 
Lide, R. F. 1991. Hydrology of a Carolina bay located on the Upper Coastal Plain, western South Carolina. Thesis. The University of Georgia, Athens, Georgia, USA.

Madison, D.M. and C.R. Shoop. 1970. Homing behavior, orientation, and home range of salamanders tagged with tantalum-182. Science $168: 1484-1487$.

Matthews, M. J., R. P. Cook, J. T. Tanacredi, and J. J. Pane. 1991. Interagency cooperation in restoring freshwater wetlands in an urban national recreation area. 151-155 in I. W. Adams and $D$. I. Leedy. Wildife conservation in metropolitan environments. National Institute for Urban Wildlife, Columbia, Maryland, USA.

McGregor, J.H., and W.R. Teska. 1989. Olfaction as an orientation mechanism in migrating Ambystoma maculatum. Copeia 1989: 779-781.

Moler, P.E., and R. Franz. 1988. Wildlife values of small, isolated wetlands in the southeastern coastal plain. Pages 234-241 in R.R. Odom, K.A. Riddleberger, and J.C. Ozier, editors. Proceedings of the third nongame and endangered wildlife symposium. Georgia Department of Natural Resources, Social Circle, Georgia, USA.

Oldham, R. S. 1966. Spring movements in the American toad, Bufo americanus. Canadian Journal of Zoology 44:63-100.

Oldham, R.S. 1967. Orienting mechanisms of the green frog, Rana clamitans. Ecology $48: 477-491$. 
Pechmann, J.H.K., D.E. Scott, J.W. Gibbons, and R.D. Semlitsch. 1989. Influence of wetland hydroperiod on diversity and abundance of metamorphosing juvenile amphibians. Wetlands Ecology and Management $1: 3-11$.

Pechmann, J.H.K., R.D. Semlitsch, R.M. Lew, and D.T. Mayack. 1984. Annual Report, FY-1983-84. Ecological studies related to the construction of the Defense Waste Processing Facility on the Savannah River Plant. NTIS Publ. SREL 17 UC-66e.

Pechmann, J.H.K., D.E. Scott, and J.N. Knox. 1985. Annual Report, FY-1985. Ecological studies related to construction of the Defense Waste Processing Facility on the Savannah River Plant. NTIS Publ. SREL 19 UC-66e.

Pechmann, J.H.R. and R.D. Semlitsch. 1986. Diel activity patterns in the breeding migrations of winter-breeding anurans. Can. $J$. 2001. 64: 1116-1120.

Pechmann, J.H.K., D.E. Scott, R.D. Semlitsch, J.P. Caldwell, I. J. Vitt, and J. W. Gibbons. 1991. Declining amphibian populations: the problem of separating human impacts from natural fluctuations. Science 253:892-895.

Phillips, J. B. 1986. Two magnetoreception pathways in a migratory salamander. Science $233: 765-767$.

Reading, C. J., J. Loman, and T. Madsen. 1991. Breeding pond fidelity in the common toad, Bufo bufo. Jounal of zoology, London $225: 201-211$. 
Reilly, S. M. 1987. Ontogeny of the hyobranchial apparatus in the salamanders Ambystoma talpoideum (Ambystomatidae) and Notophthalmus viridescens (Salamandridae): The ecological morphology of two neotenic strategies. Jounal of Morphology 191:205-214.

Rodda, G. H. , and J. B. Phillips. 1992. Navigational systems develop along similar lines in amphibians, reptiles, and birds. Ethology Ecology and Evolution 4:43-51.

Ross, T.E. 1987. A comprehensive bibliography of the Carolina bays literature. The Journal of the Elisha Mitchell scientific society $103: 28-42$.

Salvesen, D. 1990. Wetlands: mitigating and regulating development impacts. The Urban Land Institute, Washington, D.C., USA.

Savannah River Ecology Laboratory. 1980. Annual Report, FY-1980. A biological inventory of the proposed site of the Defense Wagte Processing Facility on the Savannah River Plant in Aiken, South Carolina. NTIS publ. SREL-7 UC-66e. 179 pp.

Schalles, J.F. 1979. Comparative limnology and ecosystem analysis of Carolina bay ponds on the upper coastal plain of south Carolina. Ph.D. Thesis. Emory University, Atlanta, Ga.

Schalleg, J. F., and D. J. Shure. 1989. Hydrology, community gtructure, and productivity patterns of a dystrophic Carolina bay wetland. Ecological Monographs 59:365-385.

Schlupp, I., M. Rietz, R. Podloucky, and F. M. Stolz. 1989. Pilot project Braken: preliminary results from the resettlement of adult 
toads to a substitute breeding site. 127-133 in T. E. S. Langton. Amphibians and roads. Proceedings of the Toad Tunnel Conference, Rendsburg, Federal Republic of Germany, 7-8. January 1989. ACO Polymer Products Itd, Shefford, Bedfordshire, England.

Scott, D.E., J.H.K. Pechmann, J.N. Knox, R.A. Estes, and A.M. Dancewicz. 1986. Annual Report, FY-1986. Ecological studies related to construction of the Defense Waste Processing Facility on the Savannah River Plant. NTIS Publ. SREL-32 UC-66e.

scott, D. E. 1994. The effect of larval density on adult demographic traits in Ambystoma opacum. Ecology 75:1383-1396.

Semlitsch, R.D. 1981. Terregtrial activity and summer home range of the mole salamander (Ambystoma talpoideum). Canadian Journal of Zoology 59:315-322.

Semlitsch, R.D. 1983a. structure and dynamics of twe reeding populations of the eastern tiger salamander, Ambyst a tigrinium. Copeia $1983: 608-616$

Semlitsch, R.D. 1983b. Terrestrial movements of an eastern tiger salamander, Ambystoma tigrinum. Herp Review 14: 112-113.

Semlitsch, R. D. , and J. W. Gibbons. 1985. Phenotypic variation in metamorphosis and padomorphosis in the salamander Ambystoma talpoideum. Ecology $66: 1123-1130$.

Semlitgch, R. D. 1987. Relationship of pond drying to the reproductive success of the salamander Ambystoma talpoideum. Copeia 1987:61-69. 
Semlitsch, R. D., D. E. Scott, and J. H. K. Pechmann. 1988. Time and size at metamorphosis related to adult fitness in Ambystoma talpoideum. Ecology $69: 184-192$.

Shoop, C. R., and T. L. Doty. 1972. Migratory orientation by marbled salamanders (Ambystoma opacum) near a breeding area. Behavioral Biology 7:131-136.

Sharitz, R.R., and J.W. Gibbons. 1982. The ecology of southeastern shrub bogs (pocosins) and Carolina bays: a community profile. FWS/OBS-82/04. U.S. Fish and Wildlife Service, Washington, D.C., USA.

Sinsch, U. 1990. Migration and orientation in anuran amphibians. Ethology Ecology and Evolution 2:65-79.

Sinsch, U. 1992. Structure and dynamic of a natterjack toad metapopulation (Bufo calamita). Oecologia 90:489-499.

Taylor, D.H., and K. Adler. 1973. Spatial orientation by salamanders using plane-polarized light. Science 181:285-287.

Twitty, V.C. 1959. Migration and speciation in newts. Science $130: 1735-1743$.

Twitty, v.C. 1961. Experiments on homing behavior and speciation in Taricha. Pages 415-459 in: W.F. Blair, editor. Vertebrate speciation. University of Texas Press, Austin, Texas, USA.

Vitt, I.J. 1981. Annual Report, FY-1981. A biological inventory of the proposed site of the Defense Waste Processing Facility on 
Vitt, L.J. 1981. Annual Report, FY-1981. A biological inventory of the proposed site of the Defense Waste Processing Facility on the Savannah River Plant in Aiken, south Carolina NTIS publ. SREL-8 UC-66e. $86 \mathrm{pp}$.

Vitt, L.J., R.D. Semlitsch and M.L. Cothran. 1982. Annual Report, FY-1982. A biological inventory of the proposed site of the Defense Waste Processing Facility on the Savannah River Plant in Aiken, South Carolina. NTIS publ. SREL-13 vC-66e. 146 pp.

Wake, D.B., and H.J. Morowitz. 1990. Declining amphibian populations - a global phenomenon? Report to Board on Biology, National Research Council, on workshop in Irvine, California, USA, 19-20 February 1990; reprinted Alytes 9:33-42.

Whiteman, H. H. 1994. Evolution of facultative paedomorphosis in salamanders. The Quarterly Review of Biology 69:205-221.

Williams, P.K. 1973. Seasonal movements and population dynamics of four sympatric mole salamanders, genus Ambystoma. Dissertation. Indiana University, Bloomington, Indiana, USA.

Whitford, W. G. , and A. Vinegar. 1966. Homing, survivorship, and overwintering of larvae in spotted salamanders, Ambystoma maculatum. Copeia 1966:515-519. 
TABLE III-1. Breeding population sizes and juvenile recruitment of amphibians at Sun Bay prior to construction.

1979

1980

1981

1982

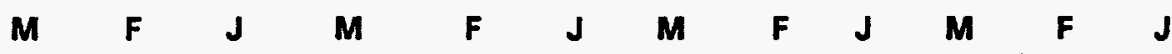

\begin{tabular}{|c|c|c|c|c|c|c|c|c|c|c|c|c|}
\hline \multicolumn{13}{|l|}{ Salamanders } \\
\hline Ambystoma opacum & $\therefore$ & -• & 0 & 11 & 10 & 19 & 27 & 7 & 31 & 8 & 5 & 93 \\
\hline A. talpoideum & $469^{b}$ & 531 & 0 & 3013 & 3300 & 0 & 113 & 53 & 0 & 100 & 974 & 0 \\
\hline A. tigrinum & $20^{b}$ & $25^{\mathrm{b}}$ & 1 & 13 & 8 & 0 & 3 & 0 & 0 & 17 & 9 & 0 \\
\hline Eurycea quadridigitata & $-\cdots$ & $-\bullet$ & 0 & 1 & 4 & 0 & 1 & 0 & 0 & 1 & 1 & 0 \\
\hline Notophthalmus & $--{ }^{*}$ & $-\bullet$ & 23 & 1271 & 1005 & 0 & 431 & 207 & 0 & 179 & 144 & 0 \\
\hline Siren intermedia & $-{ }^{-}$ & $-\circ$ & $-\odot$ & $-{ }^{\circ}$ & $-\circ$ & $-\infty$ & $-c$ & $-\circ$ & $-\infty$ & $\ldots$ & $-\infty$ & $-{ }^{\circ}$ \\
\hline \multicolumn{13}{|l|}{ Frogs and Toads } \\
\hline Acris gryllus & 3 & 0 & 0 & 2 & 7 & 0 & 1 & 1 & 0 & $0^{b}$ & $\sigma^{b}$ & 0 \\
\hline Bufo quercicus & 0 & 1 & 0 & 0 & 0 & 0 & 5 & 3 & 0 & 3 & 0 & 0 \\
\hline B. terrestris & 244 & 61 & 617 & 718 & 99 & 0 & 96 & 18 & 0 & 115 & $70^{\circ}$ & 0 \\
\hline Gastrophryne & 685 & 516 & 0 & 100 & 23 & 0 & 288 & 192 & 0 & $60^{6}$ & $18^{b}$ & 0 \\
\hline Hyla chrysoscelis & 4 & 3 & 0 & 1 & 1 & 0 & 0 & 0 & 0 & $0^{b}$ & $0^{\infty}$ & 0 \\
\hline H. femoralis & 1 & 0 & 0 & 0 & 0 & 0 & 0 & 0 & 0 & $0^{b}$ & $0^{6}$ & 0 \\
\hline H. gratiosa & 1 & 2 & 0 & 0 & 0 & 0 & 0 & 0 & 0 & $0^{b}$ & $0^{b}$ & 0 \\
\hline H. squirella & 0 & 0 & 0 & 0 & 0 & 0 & 0 & 0 & 0 & $0^{b}$ & $0^{b}$ & 0 \\
\hline Pseudacris crucifer & $145^{b}$ & 179 & 50 & 162 & 333 & 166 & 105 & 183 & 75 & 21 & 36 & 377 \\
\hline P. nigrita & $o^{b}$ & $0^{b}$ & 0 & 8 & 6 & 0 & 2 & 2 & 0 & 0 & 0 & 0 \\
\hline P. ornats & $61^{b}$ & $61^{b}$ & 31 & 165 & 138 & 19 & 73 & 45 & 55 & 62 & 55 & 423 \\
\hline Rana catesbeiana & 0 & 0 & 0 & 0 & 2 & 0 & 0 & 0 & 0 & $0^{\circ}$ & $0^{b}$ & 0 \\
\hline R. clamitans & 2 & 15 & 0 & 1 & 4 & 25 & 0 & 0 & 0 & $2^{b}$ & $3^{b}$ & 0 \\
\hline R. utricularia & $26^{b}$ & $68^{b}$ & 0 & 51 & 32 & 0 & 15 & 17 & 0 & $2^{b}$ & $2^{b}$ & 0 \\
\hline Scaphiopus holbrooki & $158^{b}$ & 100 & 58 & 451 & 361 & 0 & 5 & 4 & 0 & $16^{b}$ & $4^{b}$ & 0 \\
\hline
\end{tabular}

- Orift fence and pitfall traps not in place during breeding migration period.

Drift fence and pitfall traps not in place only during part of breeding migration period.

- Fully aquatic species, no estimates available. 
TABLE III-2. Amphibians captured during breeding migrations at sun Bay after it was filled with soil.

\begin{tabular}{|c|c|c|c|c|c|c|c|c|}
\hline & \multicolumn{2}{|c|}{1984} & \multicolumn{2}{|c|}{1985} & \multicolumn{2}{|c|}{1986} & \multicolumn{2}{|c|}{1987} \\
\hline & $\mathbf{M}$ & $\mathbf{F}$ & $\mathbf{M}$ & $\mathbf{F}$ & $\mathbf{M}$ & $\mathbf{F}$ & $\mathbf{M}$ & $\mathbf{F}$ \\
\hline \multicolumn{9}{|l|}{ Salamanders } \\
\hline Ambystoma opacum & $1^{8}$ & $0^{b}$ & $0^{b}$ & $0^{b}$ & $0^{b}$ & $o^{b}$ & 0 & 0 \\
\hline A. talpoideum & $20^{b}$ & $14^{b}$ & $0^{b}$ & $1^{b}$ & 4 & 5 & 0 & 0 \\
\hline A. tigrinum & $0^{b}$ & $0^{b}$ & $0^{b}$ & $0^{b}$ & 0 & 0 & 0 & 0 \\
\hline Eurycea quadridigitata & $--^{\circ}$ & $-\infty$ & $-\infty$ & $\ldots$ & $-\infty$ & $-{ }^{\circ}$ & 0 & 0 \\
\hline Notophthalmus viridescens & $13^{b}$ & $5^{b}$ & $0^{b}$ & $0^{b}$ & $0^{b}$ & $0^{b}$ & 0 & 0 \\
\hline Siren intermedia & $-c$ & $--^{\mathrm{c}}$ & $--c$ & $--^{\circ}$ & $\therefore$ & $--^{c}$ & $--c$ & $-c^{\circ}$ \\
\hline \multicolumn{9}{|l|}{ Frogs and Toads } \\
\hline Acris gryllus & $0^{b}$ & $0^{b}$ & $0^{b}$ & $0^{b}$ & 0 & 0 & 0 & 0 \\
\hline Bufo quercicus & $0^{b}$ & $0^{b}$ & $0^{b}$ & $0^{b}$ & 0 & 0 & 0 & 0 \\
\hline B. terrestris & $9^{b}$ & $3^{b}$ & 3 & 3 & 0 & 0 & 0 & 0 \\
\hline Gastrophryne carolinensis & $2^{b}$ & 96 & $2^{\circ}$ & $2^{b}$ & $0^{\infty}$ & $0^{b}$ & 0 & 0 \\
\hline Hyla chrysoscelis & $O^{b}$ & $0^{b}$ & $0^{b}$ & $0^{b}$ & 0 & 0 & 0 & 0 \\
\hline H. femoralis & $0^{b}$ & $0^{b}$ & $0^{b}$ & $0^{b}$ & 0 & 0 & 0 & 0 \\
\hline H. gratiosa & $0^{b}$ & $0^{b}$ & $o^{b}$ & $0^{b}$ & 0 & 0 & 0 & 0 \\
\hline H. squirella & $O^{b}$ & $0^{b}$ & $0^{b}$ & $0^{b}$ & 0 & 0 & 0 & 0 \\
\hline Pseudacris crucifer & $3^{b}$ & $3^{b}$ & $1^{b}$ & $0^{b}$ & 0 & 0 & 0 & 0 \\
\hline P. nigrita & $0^{b}$ & $0^{b}$ & $0^{b}$ & $0^{b}$ & 0 & 0 & 0 & 0 \\
\hline P. ornata & $8^{b}$ & $2^{b}$ & $0^{b}$ & $0^{b}$ & 0 & 0 & 0 & 0 \\
\hline Rana catesbeiana & $0^{b}$ & $0^{b}$ & $0^{b}$ & $0^{b}$ & 0 & 0 & 0 & 0 \\
\hline R. clamitans & $1^{b}$ & $0^{b}$ & $0^{b}$ & $0^{b}$ & 0 & 0 & 0 & 0 \\
\hline R. utricularia & $10^{b}$ & $3^{b}$ & $0^{b}$ & $0^{b}$ & $0^{b}$ & $1^{b}$ & 0 & 1 \\
\hline Scaphiopus holbrooki & $5^{b}$ & $3^{b}$ & 10 & $0^{\circ}$ & $1^{6}$ & $2^{b}$ & 0 & 0 \\
\hline
\end{tabular}

- Drift fence and pitfall traps not in place during breeding migration period.

'Drift fence and pitfall traps not in place only during part of breeding migration period.

${ }^{\circ}$ Fully aquatic species, no estimates available. 
TABLE III-3. PART IA. Adult Breeding Populations at the Refuge Ponds and Sun Bay.

\begin{tabular}{|c|c|c|c|c|c|c|}
\hline \multirow{2}{*}{$\begin{array}{l} \\
1982-1983 \\
\text { Pond A } \\
\text { Pond B } \\
\text { Pond C } \\
\text { Pond D } \\
\text { Sun Bay } \\
\end{array}$} & \multicolumn{2}{|c|}{ Bufo terrestris } & \multicolumn{2}{|c|}{ Pseudacris ornata } & \multicolumn{2}{|c|}{ Rana utricularia } \\
\hline & $\begin{array}{c}\operatorname{male} \\
1 \\
0 \\
0 \\
0 \\
0\end{array}$ & $\begin{array}{c}\text { female } \\
3 \\
2 \\
2 \\
1 \\
0\end{array}$ & $\begin{array}{c}\text { male } \\
0 \\
0 \\
0 \\
0 \\
0\end{array}$ & $\begin{array}{c}\text { female } \\
0 \\
0 \\
0 \\
0 \\
0\end{array}$ & $\begin{array}{c}\operatorname{male} \\
0 \\
0 \\
0 \\
0 \\
0 \\
\end{array}$ & $\begin{array}{c}\text { female } \\
0 \\
0 \\
0 \\
0 \\
0 \\
\end{array}$ \\
\hline $\begin{array}{l}1983-1984 \\
\text { Pond A } \\
\text { Pond B } \\
\text { Pond C } \\
\text { Pond D } \\
\text { Sun Bay } \\
\end{array}$ & $\begin{array}{c}\operatorname{male} \\
4 \\
6 \\
5 \\
1 \\
9 \\
\end{array}$ & $\begin{array}{c}\text { female } \\
3 \\
1 \\
1 \\
2 \\
3 \\
\end{array}$ & $\begin{array}{c}\text { male } \\
0 \\
1 \\
0 \\
1 \\
8 \\
\end{array}$ & $\begin{array}{c}\text { female } \\
0 \\
0 \\
2 \\
1 \\
2 \\
\end{array}$ & $\begin{array}{c}\text { male } \\
3 \\
7 \\
2 \\
1 \\
10 \\
\end{array}$ & $\begin{array}{c}\text { female } \\
2 \\
8 \\
0 \\
0 \\
3 \\
\end{array}$ \\
\hline $\begin{array}{l}1984-1985 \\
\text { Pond A } \\
\text { Pond B } \\
\text { Pond C } \\
\text { Pond D } \\
\text { Sun Bay }\end{array}$ & $\begin{array}{l}\text { male } \\
27 \\
37 \\
11 \\
28 \\
3\end{array}$ & $\begin{array}{c}\text { female } \\
3 \\
19 \\
6 \\
9 \\
3\end{array}$ & $\begin{array}{c}\text { male } \\
0 \\
0 \\
1 \\
0 \\
0\end{array}$ & $\begin{array}{c}\text { female } \\
0 \\
2 \\
1 \\
1 \\
0\end{array}$ & $\begin{array}{c}\operatorname{male} \\
6 \\
5 \\
2 \\
2 \\
0\end{array}$ & $\begin{array}{c}\text { female } \\
1 \\
1 \\
0 \\
0 \\
0\end{array}$ \\
\hline $\begin{array}{l}1985-1986 \\
\text { Pond A } \\
\text { Pond B } \\
\text { Pond C } \\
\text { Pond D } \\
\text { Sun Bay }\end{array}$ & $\begin{array}{l}\text { male } \\
30 \\
49 \\
-- \\
23 \\
0\end{array}$ & $\begin{array}{c}\text { female } \\
10 \\
22 \\
-1 \\
11 \\
0\end{array}$ & $\begin{array}{c}\text { male } \\
0 \\
3 \\
-- \\
1 \\
0\end{array}$ & $\begin{array}{c}\text { female } \\
0 \\
0 \\
- \\
0 \\
0\end{array}$ & $\begin{array}{l}\text { male } \\
36 \\
36 \\
-13 \\
0\end{array}$ & $\begin{array}{c}\text { female } \\
7 \\
6 \\
-1 \\
3 \\
1\end{array}$ \\
\hline $\begin{array}{l}1986-1987 \\
\text { Pond A } \\
\text { Pond B } \\
\text { Pond C } \\
\text { Pond D } \\
\text { Sun Bay } \\
\end{array}$ & $\begin{array}{l}\text { male } \\
19 \\
12 \\
-- \\
4 \\
0\end{array}$ & $\begin{array}{c}\text { female } \\
4 \\
2 \\
- \\
7 \\
0 \\
\end{array}$ & $\begin{array}{c}\operatorname{male} \\
0 \\
0 \\
- \\
0 \\
0\end{array}$ & $\begin{array}{c}\text { female } \\
0 \\
0 \\
- \\
0 \\
0\end{array}$ & $\begin{array}{c}\text { male } \\
14 \\
1 \\
-1 \\
0 \\
0\end{array}$ & $\begin{array}{c}\text { female } \\
8 \\
2 \\
- \\
0 \\
1\end{array}$ \\
\hline $\begin{array}{l}1987-1988 \\
\text { Pond A } \\
\text { Pond B } \\
\text { Pond C } \\
\text { Pond D } \\
\text { Sun Bay }\end{array}$ & $\begin{array}{c}\operatorname{male} \\
5 \\
11 \\
-- \\
16 \\
--\end{array}$ & $\begin{array}{c}\text { female } \\
8 \\
3 \\
-10 \\
--\end{array}$ & $\begin{array}{c}\text { male } \\
1 \\
4 \\
-\frac{2}{2} \\
--\end{array}$ & $\begin{array}{c}\text { female } \\
0 \\
1 \\
-2 \\
2 \\
--\end{array}$ & $\begin{array}{c}\text { male } \\
13 \\
3 \\
-1 \\
--\end{array}$ & $\begin{array}{c}\text { female } \\
0 \\
1 \\
-0 \\
0 \\
--\end{array}$ \\
\hline $\begin{array}{l}1988-1989 \\
\text { Pond A } \\
\text { Pond B } \\
\text { Pond C } \\
\text { Pond D } \\
\text { Sun Bay } \\
\end{array}$ & $\begin{array}{c}\operatorname{male} \\
9 \\
8 \\
-- \\
18 \\
-- \\
\end{array}$ & $\begin{array}{c}\text { female } \\
4 \\
5 \\
-5 \\
8 \\
--\end{array}$ & $\begin{array}{c}\text { male } \\
0 \\
4 \\
-- \\
0 \\
--\end{array}$ & $\begin{array}{c}\text { female } \\
0 \\
1 \\
- \\
0 \\
--\end{array}$ & $\begin{array}{c}\text { male } \\
21 \\
6 \\
-- \\
--\end{array}$ & $\begin{array}{c}\text { female } \\
1 \\
0 \\
- \\
0 \\
--\end{array}$ \\
\hline $\begin{array}{l}1989-1990 \\
\text { Pond A } \\
\text { Pond B } \\
\text { Pond C } \\
\text { Pond D } \\
\text { Sun Bay }\end{array}$ & $\begin{array}{c}\text { male } \\
9 \\
5 \\
-- \\
26 \\
--\end{array}$ & $\begin{array}{c}\text { female } \\
4 \\
2 \\
-\overline{10} \\
--\end{array}$ & $\begin{array}{c}\text { male } \\
0 \\
0 \\
-0 \\
--\end{array}$ & $\begin{array}{c}\text { female } \\
0 \\
0 \\
- \\
0 \\
--\end{array}$ & $\begin{array}{c}\operatorname{male} \\
4 \\
2 \\
-0 \\
--\end{array}$ & $\begin{array}{c}\text { female } \\
3 \\
2 \\
-- \\
0 \\
--\end{array}$ \\
\hline $\begin{array}{l}1990-1991 \\
\text { Pond A } \\
\text { Pond B } \\
\text { Pond C } \\
\text { Pond D } \\
\text { Sun Bay }\end{array}$ & $\begin{array}{l}\operatorname{male} \\
19 \\
23 \\
-- \\
23 \\
--\end{array}$ & $\begin{array}{c}\text { female } \\
5 \\
11 \\
-5 \\
11 \\
--\end{array}$ & $\begin{array}{c}\operatorname{male} \\
0 \\
0 \\
-- \\
0 \\
--\end{array}$ & $\begin{array}{c}\text { female } \\
0 \\
0 \\
- \\
0 \\
--\end{array}$ & $\begin{array}{c}\operatorname{male} \\
4 \\
2 \\
-1 \\
1 \\
--\end{array}$ & $\begin{array}{c}\text { female } \\
3 \\
1 \\
- \\
0 \\
--\end{array}$ \\
\hline
\end{tabular}


TABLE III-3. PART IB.

\begin{tabular}{|c|c|c|c|c|c|c|}
\hline & \multicolumn{2}{|c|}{ Hyla crucifer } & \multicolumn{2}{|c|}{ Pseudacris nigrita } & \multicolumn{2}{|c|}{ Rana catesbeiana } \\
\hline $\begin{array}{l}\text { 1982-1983 } \\
\text { Pond A } \\
\text { Pond B } \\
\text { Pond C } \\
\text { Pond D } \\
\text { Sun Bay }\end{array}$ & $\begin{array}{c}\operatorname{male} \\
0 \\
0 \\
0 \\
0 \\
0\end{array}$ & $\begin{array}{c}\text { female } \\
0 \\
0 \\
0 \\
0 \\
0\end{array}$ & $\begin{array}{c}\text { male } \\
0 \\
0 \\
0 \\
0 \\
0\end{array}$ & $\begin{array}{c}\text { female } \\
0 \\
0 \\
0 \\
0 \\
0\end{array}$ & $\begin{array}{c}\text { male } \\
0 \\
0 \\
0 \\
0 \\
0\end{array}$ & $\begin{array}{c}\text { female } \\
0 \\
0 \\
0 \\
0 \\
0\end{array}$ \\
\hline $\begin{array}{l}1983-1984 \\
\text { Pond A } \\
\text { Pond B } \\
\text { Pond C } \\
\text { Pond D } \\
\text { Sun Bay }\end{array}$ & $\begin{array}{c}\text { male } \\
1 \\
5 \\
1 \\
0 \\
3\end{array}$ & $\begin{array}{c}\text { female } \\
1 \\
8 \\
3 \\
1 \\
3 \\
\end{array}$ & $\begin{array}{c}\text { male } \\
0 \\
0 \\
0 \\
0 \\
0 \\
\end{array}$ & $\begin{array}{c}\text { female } \\
0 \\
0 \\
0 \\
0 \\
0\end{array}$ & $\begin{array}{c}\text { male } \\
0 \\
0 \\
0 \\
0 \\
0\end{array}$ & $\begin{array}{c}\text { female } \\
0 \\
1 \\
0 \\
0 \\
0\end{array}$ \\
\hline $\begin{array}{l}1984-1985 \\
\text { Pond A } \\
\text { Pond B } \\
\text { Pond C } \\
\text { Pond D } \\
\text { Sun Bay }\end{array}$ & $\begin{array}{c}\text { male } \\
1 \\
2 \\
6 \\
5 \\
1\end{array}$ & $\begin{array}{c}\text { female } \\
2 \\
5 \\
9 \\
5 \\
0\end{array}$ & $\begin{array}{c}\text { male } \\
0 \\
1 \\
0 \\
0 \\
0\end{array}$ & $\begin{array}{c}\text { female } \\
0 \\
0 \\
0 \\
1 \\
0\end{array}$ & $\begin{array}{c}\operatorname{male} \\
0 \\
0 \\
0 \\
0 \\
0\end{array}$ & $\begin{array}{c}\text { female } \\
0 \\
0 \\
0 \\
0 \\
0\end{array}$ \\
\hline $\begin{array}{l}1985-1986 \\
\text { Pond A } \\
\text { Pond B } \\
\text { Pond C } \\
\text { Pond D } \\
\text { Sun Bay } \\
\end{array}$ & $\begin{array}{l}\text { male } \\
12 \\
54 \\
-19 \\
19 \\
0\end{array}$ & $\begin{array}{c}\text { female } \\
13 \\
61 \\
-0 \\
15 \\
0 \\
\end{array}$ & $\begin{array}{c}\text { male } \\
0 \\
1 \\
-- \\
0 \\
0 \\
\end{array}$ & $\begin{array}{c}\text { female } \\
0 \\
0 \\
-- \\
2 \\
0 \\
\end{array}$ & $\begin{array}{c}\text { male } \\
2 \\
0 \\
-0 \\
0 \\
0\end{array}$ & $\begin{array}{c}\text { female } \\
1 \\
0 \\
-- \\
0 \\
0\end{array}$ \\
\hline $\begin{array}{l}\text { 1986-1987 } \\
\text { Pond A } \\
\text { Pond B } \\
\text { Pond C } \\
\text { Pond D } \\
\text { Sun Bay } \\
\end{array}$ & $\begin{array}{c}\operatorname{male} \\
2 \\
2 \\
-- \\
3 \\
--\end{array}$ & $\begin{array}{c}\text { female } \\
1 \\
0 \\
-- \\
2 \\
- \\
\end{array}$ & $\begin{array}{c}\text { male } \\
0 \\
0 \\
-- \\
0 \\
-- \\
\end{array}$ & $\begin{array}{c}\text { female } \\
0 \\
0 \\
- \\
0 \\
-- \\
\end{array}$ & $\begin{array}{c}\operatorname{male} \\
0 \\
0 \\
-- \\
0 \\
-- \\
\end{array}$ & $\begin{array}{c}\text { female } \\
1 \\
0 \\
-0 \\
0 \\
-\end{array}$ \\
\hline $\begin{array}{l}1987-1988 \\
\text { Pond A } \\
\text { Pond B } \\
\text { Pond C } \\
\text { Pond D } \\
\text { Sun Bay }\end{array}$ & $\begin{array}{c}\text { male } \\
10 \\
3 \\
-- \\
0 \\
--\end{array}$ & $\begin{array}{c}\text { female } \\
12 \\
5 \\
- \\
0 \\
--\end{array}$ & $\begin{array}{c}\text { male } \\
0 \\
0 \\
-- \\
0 \\
-- \\
\end{array}$ & $\begin{array}{c}\text { female } \\
0 \\
0 \\
- \\
0 \\
--\end{array}$ & $\begin{array}{c}\text { male } \\
0 \\
0 \\
--1 \\
--\end{array}$ & $\begin{array}{c}\text { female } \\
0 \\
0 \\
-- \\
0 \\
--\end{array}$ \\
\hline $\begin{array}{l}1988-1989 \\
\text { Pond A } \\
\text { Pond B } \\
\text { Pond C } \\
\text { Pond D } \\
\text { Sun Bay }\end{array}$ & $\begin{array}{c}\operatorname{male} \\
14 \\
2 \\
-- \\
-3 \\
--\end{array}$ & $\begin{array}{c}\text { female } \\
15 \\
5 \\
-- \\
3 \\
--\end{array}$ & $\begin{array}{c}\text { male } \\
0 \\
0 \\
-- \\
0 \\
-- \\
\end{array}$ & $\begin{array}{c}\text { female } \\
0 \\
0 \\
-- \\
0 \\
-- \\
\end{array}$ & $\begin{array}{c}\operatorname{male} \\
0 \\
0 \\
-- \\
0 \\
--\end{array}$ & $\begin{array}{c}\text { female } \\
0 \\
0 \\
-- \\
0 \\
-- \\
\end{array}$ \\
\hline $\begin{array}{l}1989-1990 \\
\text { Pond A } \\
\text { Pond B } \\
\text { Pond C } \\
\text { Pond D } \\
\text { Sun Bay } \\
\end{array}$ & $\begin{array}{c}\operatorname{male} \\
10 \\
0 \\
-- \\
5 \\
--\end{array}$ & $\begin{array}{c}\text { female } \\
9 \\
5 \\
-5 \\
-- \\
\end{array}$ & $\begin{array}{c}\text { male } \\
0 \\
0 \\
-- \\
0 \\
-- \\
\end{array}$ & $\begin{array}{c}\text { female } \\
0 \\
0 \\
-0 \\
0 \\
-- \\
\end{array}$ & $\begin{array}{c}\operatorname{male} \\
0 \\
0 \\
-- \\
0 \\
--\end{array}$ & $\begin{array}{c}\text { female } \\
1 \\
0 \\
-- \\
0 \\
--\end{array}$ \\
\hline $\begin{array}{l}1990-1991 \\
\text { Pond A } \\
\text { Pond B } \\
\text { Pond C } \\
\text { Pond D } \\
\text { Sun Bay }\end{array}$ & $\begin{array}{c}\operatorname{male} \\
0 \\
0 \\
-- \\
-3 \\
--\end{array}$ & $\begin{array}{c}\text { female } \\
0 \\
1 \\
-- \\
-3 \\
--\end{array}$ & $\begin{array}{c}\text { male } \\
0 \\
0 \\
-- \\
0 \\
--\end{array}$ & $\begin{array}{c}\text { female } \\
0 \\
0 \\
-- \\
0 \\
--\end{array}$ & $\begin{array}{c}\operatorname{male} \\
0 \\
0 \\
-- \\
0 \\
--\end{array}$ & $\begin{array}{c}\text { female } \\
0 \\
0 \\
- \\
0 \\
--\end{array}$ \\
\hline
\end{tabular}


TABLE III-3. PART IC.

\begin{tabular}{|c|c|c|c|c|c|c|}
\hline \multirow[b]{2}{*}{$\begin{array}{l}1982-1983 \\
\text { Pond A } \\
\text { Pond B } \\
\text { Pond C } \\
\text { Pond D } \\
\text { Sun Bay }\end{array}$} & \multicolumn{2}{|c|}{$\begin{array}{l}\text { Gastrophryne } \\
\text { carolinensis }\end{array}$} & \multicolumn{2}{|c|}{$\begin{array}{l}\text { Rana } \\
\text { clamitans }\end{array}$} & \multicolumn{2}{|c|}{$\begin{array}{l}\text { Scaphiopus } \\
\text { holbrooki }\end{array}$} \\
\hline & $\begin{array}{c}\text { male } \\
6 \\
11 \\
7 \\
5 \\
0 \\
\end{array}$ & $\begin{array}{c}\text { female } \\
5 \\
8 \\
1 \\
4 \\
0 \\
\end{array}$ & $\begin{array}{c}\text { male } \\
0 \\
0 \\
0 \\
0 \\
0 \\
\end{array}$ & $\begin{array}{c}\text { female } \\
0 \\
0 \\
0 \\
0 \\
0 \\
\end{array}$ & $\begin{array}{c}\text { male } \\
0 \\
0 \\
2 \\
0 \\
0 \\
\end{array}$ & $\begin{array}{c}\text { female } \\
0 \\
1 \\
0 \\
3 \\
0 \\
\end{array}$ \\
\hline $\begin{array}{l}\text { 1983-1984 } \\
\text { Pond A } \\
\text { Pond B } \\
\text { Pond C } \\
\text { Pond D } \\
\text { Sun Bay } \\
\end{array}$ & $\begin{array}{c}\text { male } \\
17 \\
8 \\
2 \\
12 \\
2 \\
\end{array}$ & $\begin{array}{c}\text { female } \\
7 \\
9 \\
2 \\
9 \\
1 \\
\end{array}$ & $\begin{array}{c}\operatorname{male} \\
0 \\
1 \\
2 \\
1 \\
1 \\
\end{array}$ & $\begin{array}{c}\text { female } \\
0 \\
3 \\
1 \\
1 \\
0 \\
\end{array}$ & $\begin{array}{c}\text { male } \\
0 \\
5 \\
8 \\
1 \\
5 \\
\end{array}$ & $\begin{array}{c}\text { female } \\
1 \\
1 \\
5 \\
1 \\
3 \\
\end{array}$ \\
\hline $\begin{array}{l}\text { 1984-1985 } \\
\text { Pond A } \\
\text { Pond B } \\
\text { Pond C } \\
\text { Pond D } \\
\text { Sun Bay } \\
\end{array}$ & $\begin{array}{c}\text { male } \\
14 \\
5 \\
5 \\
11 \\
2 \\
\end{array}$ & $\begin{array}{c}\text { female } \\
19 \\
3 \\
3 \\
5 \\
2 \\
\end{array}$ & $\begin{array}{c}\text { male } \\
5 \\
2 \\
1 \\
0 \\
0 \\
\end{array}$ & $\begin{array}{c}\text { female } \\
0 \\
2 \\
0 \\
0 \\
0 \\
\end{array}$ & $\begin{array}{c}\operatorname{male} \\
0 \\
4 \\
1 \\
2 \\
1\end{array}$ & $\begin{array}{c}\text { female } \\
0 \\
2 \\
0 \\
2 \\
0\end{array}$ \\
\hline $\begin{array}{l}1985-1986 \\
\text { Pond A } \\
\text { Pond B } \\
\text { Pond C } \\
\text { Pond D } \\
\text { Sun Bay } \\
\end{array}$ & $\begin{array}{c}\operatorname{male} \\
12 \\
6 \\
-- \\
2 \\
0\end{array}$ & $\begin{array}{c}\text { female } \\
17 \\
4 \\
- \\
0 \\
0 \\
\end{array}$ & $\begin{array}{c}\text { male } \\
1 \\
2 \\
-0 \\
0 \\
0\end{array}$ & $\begin{array}{c}\text { female } \\
2 \\
2 \\
-1 \\
1 \\
0 \\
\end{array}$ & $\begin{array}{c}\operatorname{male} \\
0 \\
2 \\
-1 \\
1 \\
1\end{array}$ & $\begin{array}{c}\text { female } \\
0 \\
3 \\
-2 \\
1 \\
2 \\
\end{array}$ \\
\hline $\begin{array}{l}\text { 1986-1987 } \\
\text { Pond A } \\
\text { Pond B } \\
\text { Pond C } \\
\text { Pond D } \\
\text { Sun Bay } \\
\end{array}$ & $\begin{array}{c}\operatorname{male} \\
7 \\
2 \\
-- \\
7 \\
-- \\
\end{array}$ & $\begin{array}{c}\text { female } \\
11 \\
4 \\
-- \\
2 \\
-- \\
\end{array}$ & $\begin{array}{c}\text { male } \\
0 \\
0 \\
-- \\
0 \\
--\end{array}$ & $\begin{array}{c}\text { female } \\
0 \\
0 \\
-- \\
0 \\
--\end{array}$ & $\begin{array}{c}\operatorname{male} \\
4 \\
4 \\
-- \\
0 \\
--\end{array}$ & $\begin{array}{c}\text { female } \\
1 \\
3 \\
-1 \\
0 \\
--\end{array}$ \\
\hline $\begin{array}{l}1987-1988 \\
\text { Pond A } \\
\text { Pond B } \\
\text { Pond C } \\
\text { Pond D } \\
\text { Sun Bay } \\
\end{array}$ & $\begin{array}{c}\operatorname{male} \\
8 \\
9 \\
-- \\
-2 \\
--\end{array}$ & $\begin{array}{c}\text { female } \\
5 \\
2 \\
-- \\
5 \\
-- \\
\end{array}$ & $\begin{array}{c}\operatorname{male} \\
0 \\
0 \\
-- \\
0 \\
--\end{array}$ & $\begin{array}{c}\text { female } \\
0 \\
1 \\
-- \\
0 \\
--\end{array}$ & $\begin{array}{c}\operatorname{male} \\
0 \\
2 \\
- \\
0 \\
--\end{array}$ & $\begin{array}{c}\text { Eemale } \\
2 \\
2 \\
- \\
0 \\
--\end{array}$ \\
\hline $\begin{array}{l}1988-1989 \\
\text { Pond A } \\
\text { Pond B } \\
\text { Pond C } \\
\text { Pond D } \\
\text { Sun Bay } \\
\end{array}$ & $\begin{array}{c}\text { male } \\
8 \\
1 \\
-- \\
17 \\
-- \\
\end{array}$ & $\begin{array}{c}\text { female } \\
4 \\
3 \\
-- \\
18 \\
-- \\
\end{array}$ & $\begin{array}{c}\operatorname{male} \\
0 \\
0 \\
-- \\
0 \\
-- \\
\end{array}$ & $\begin{array}{c}\text { female } \\
0 \\
0 \\
- \\
2 \\
-- \\
\end{array}$ & $\begin{array}{c}\operatorname{male} \\
1 \\
0 \\
-- \\
0 \\
-- \\
\end{array}$ & $\begin{array}{c}\text { female } \\
0 \\
1 \\
-- \\
0 \\
-- \\
\end{array}$ \\
\hline $\begin{array}{l}1989-1990 \\
\text { Pond A } \\
\text { Pond B } \\
\text { Pond C } \\
\text { Pond D } \\
\text { Sun Bay }\end{array}$ & $\begin{array}{c}\operatorname{male}_{10} \\
4 \\
-- \\
2 \\
--\end{array}$ & $\begin{array}{c}\text { female } \\
\frac{1}{3} \\
-\frac{8}{8} \\
--\end{array}$ & $\begin{array}{c}\operatorname{male} \\
0 \\
0 \\
-1 \\
0 \\
--\end{array}$ & $\begin{array}{c}\text { female } \\
1 \\
0 \\
-0 \\
0 \\
--\end{array}$ & $\begin{array}{c}\operatorname{male} \\
0 \\
0 \\
-- \\
0 \\
--\end{array}$ & $\begin{array}{c}\text { female } \\
0 \\
1 \\
-- \\
0 \\
--\end{array}$ \\
\hline $\begin{array}{l}1990-1991 \\
\text { Pond A } \\
\text { Pond B } \\
\text { Pond C } \\
\text { Pond D } \\
\text { Sun Bay }\end{array}$ & $\begin{array}{l}\text { male } \\
11 \\
7 \\
-- \\
33 \\
--\end{array}$ & $\begin{array}{c}\text { female } \\
6 \\
14 \\
-- \\
49 \\
--\end{array}$ & $\begin{array}{c}\operatorname{male} \\
0 \\
0 \\
-- \\
1 \\
--\end{array}$ & $\begin{array}{c}\text { female } \\
0 \\
0 \\
-- \\
0 \\
--\end{array}$ & $\begin{array}{c}\text { male } \\
0 \\
0 \\
-- \\
0 \\
--\end{array}$ & $\begin{array}{c}\text { female } \\
0 \\
2 \\
-- \\
0 \\
--\end{array}$ \\
\hline
\end{tabular}


TABLE III-3. PART ID.

\begin{tabular}{|c|c|c|c|c|c|c|}
\hline \multirow{2}{*}{$\begin{array}{l}\text { 1982-1983 } \\
\text { Pond A } \\
\text { Pond B } \\
\text { Pond C } \\
\text { Pond D } \\
\text { Sun Bay } \\
\end{array}$} & \multicolumn{2}{|c|}{ Hyla cinerea } & \multicolumn{2}{|c|}{ Hyla gratiosa } & \multicolumn{2}{|c|}{ Byla femoralis } \\
\hline & $\begin{array}{c}\operatorname{male} \\
0 \\
0 \\
0 \\
0 \\
0\end{array}$ & $\begin{array}{c}\text { female } \\
0 \\
0 \\
0 \\
0 \\
0\end{array}$ & $\begin{array}{c}\operatorname{male} \\
0 \\
0 \\
0 \\
0 \\
0\end{array}$ & $\begin{array}{c}\text { female } \\
0 \\
0 \\
0 \\
0 \\
0\end{array}$ & $\begin{array}{c}\text { male } \\
0 \\
3 \\
0 \\
0 \\
0\end{array}$ & $\begin{array}{c}\text { female } \\
0 \\
7 \\
0 \\
0 \\
0\end{array}$ \\
\hline $\begin{array}{l}\text { 1983-1984 } \\
\text { Pond A } \\
\text { Pond B } \\
\text { Pond C } \\
\text { Pond D } \\
\text { Sun Bay }\end{array}$ & $\begin{array}{c}\text { male } \\
0 \\
0 \\
0 \\
0 \\
0\end{array}$ & $\begin{array}{c}\text { female } \\
0 \\
0 \\
0 \\
0 \\
0 \\
\end{array}$ & $\begin{array}{c}\text { male } \\
0 \\
0 \\
0 \\
0 \\
0\end{array}$ & $\begin{array}{c}\text { female } \\
0 \\
0 \\
0 \\
0 \\
0\end{array}$ & $\begin{array}{c}\operatorname{male} \\
0 \\
1 \\
0 \\
0 \\
0\end{array}$ & $\begin{array}{c}\text { female } \\
0 \\
1 \\
0 \\
1 \\
0\end{array}$ \\
\hline $\begin{array}{l}\text { 1984-1985 } \\
\text { Pond A } \\
\text { Pond B } \\
\text { Pond C } \\
\text { Pond D } \\
\text { Sun Bay }\end{array}$ & $\begin{array}{c}\operatorname{male} \\
0 \\
0 \\
0 \\
0 \\
0\end{array}$ & $\begin{array}{c}\text { female } \\
0 \\
0 \\
0 \\
0 \\
0 \\
\end{array}$ & $\begin{array}{c}\operatorname{male} \\
0 \\
0 \\
0 \\
0 \\
0\end{array}$ & $\begin{array}{c}\text { female } \\
0 \\
0 \\
0 \\
0 \\
0\end{array}$ & $\begin{array}{c}\operatorname{male} \\
0 \\
1 \\
0 \\
0 \\
0\end{array}$ & $\begin{array}{c}\text { female } \\
1 \\
1 \\
0 \\
0 \\
0\end{array}$ \\
\hline $\begin{array}{l}1985-1986 \\
\text { Pond A } \\
\text { Pond B } \\
\text { Pond C } \\
\text { Pond D } \\
\text { Sun Bay } \\
\end{array}$ & $\begin{array}{c}\text { male } \\
0 \\
0 \\
0 \\
0 \\
0 \\
\end{array}$ & $\begin{array}{c}\text { female } \\
0 \\
0 \\
0 \\
0 \\
0 \\
\end{array}$ & $\begin{array}{c}\text { male } \\
0 \\
0 \\
0 \\
0 \\
0\end{array}$ & $\begin{array}{c}\text { female } \\
0 \\
0 \\
0 \\
0 \\
0 \\
\end{array}$ & $\begin{array}{c}\operatorname{male} \\
0 \\
0 \\
0 \\
1 \\
0\end{array}$ & $\begin{array}{c}\text { female } \\
0 \\
0 \\
0 \\
0 \\
0 \\
\end{array}$ \\
\hline $\begin{array}{l}\text { 1986-1987 } \\
\text { Pond A } \\
\text { Pond B } \\
\text { Pond C } \\
\text { Pond D } \\
\text { Sun Bay }\end{array}$ & $\begin{array}{c}\operatorname{male} \\
0 \\
0 \\
-- \\
0 \\
--\end{array}$ & $\begin{array}{c}\text { female } \\
0 \\
0 \\
- \\
0 \\
--\end{array}$ & $\begin{array}{c}\operatorname{male} \\
0 \\
0 \\
-- \\
0 \\
--\end{array}$ & $\begin{array}{c}\text { female } \\
0 \\
0 \\
- \\
0 \\
-\end{array}$ & $\begin{array}{c}\operatorname{male} \\
0 \\
0 \\
-- \\
0 \\
--\end{array}$ & $\begin{array}{c}\text { female } \\
0 \\
0 \\
-- \\
0 \\
--\end{array}$ \\
\hline $\begin{array}{l}\text { 1987-1988 } \\
\text { Pond A } \\
\text { Pond B } \\
\text { Pond C } \\
\text { Pond D } \\
\text { Sun Bay }\end{array}$ & $\begin{array}{c}\operatorname{male} \\
0 \\
0 \\
-- \\
0 \\
--\end{array}$ & $\begin{array}{c}\text { female } \\
0 \\
0 \\
-- \\
0 \\
--\end{array}$ & $\begin{array}{c}\operatorname{male} \\
0 \\
0 \\
-- \\
0 \\
--\end{array}$ & $\begin{array}{c}\text { female } \\
0 \\
0 \\
- \\
0 \\
--\end{array}$ & $\begin{array}{c}\operatorname{male} \\
0 \\
0 \\
-- \\
0 \\
--\end{array}$ & $\begin{array}{c}\text { female } \\
0 \\
0 \\
-- \\
0 \\
--\end{array}$ \\
\hline $\begin{array}{l}1988-1989 \\
\text { Pond A } \\
\text { Pond B } \\
\text { Pond C } \\
\text { Pond D } \\
\text { Sun Bay } \\
\end{array}$ & $\begin{array}{c}\operatorname{male} \\
0 \\
0 \\
-- \\
0 \\
--\end{array}$ & $\begin{array}{c}\text { female } \\
0 \\
0 \\
- \\
0 \\
--\end{array}$ & $\begin{array}{c}\text { male } \\
0 \\
0 \\
-- \\
0 \\
--\end{array}$ & $\begin{array}{c}\text { female } \\
0 \\
0 \\
- \\
0 \\
--\end{array}$ & $\begin{array}{c}\operatorname{male} \\
0 \\
0 \\
-- \\
0 \\
-- \\
\end{array}$ & $\begin{array}{c}\text { female } \\
0 \\
0 \\
- \\
0 \\
--\end{array}$ \\
\hline $\begin{array}{l}1989-1990 \\
\text { Pond A } \\
\text { Pond B } \\
\text { Pond C } \\
\text { Pond D } \\
\text { Sun Bay } \\
\end{array}$ & $\begin{array}{c}\operatorname{male} \\
0 \\
0 \\
- \\
0 \\
-- \\
\end{array}$ & $\begin{array}{c}\text { female } \\
0 \\
0 \\
-- \\
0 \\
-- \\
\end{array}$ & $\begin{array}{c}\operatorname{male} \\
0 \\
0 \\
-- \\
0 \\
-- \\
\end{array}$ & $\begin{array}{c}\text { female } \\
0 \\
0 \\
-- \\
0 \\
-- \\
\end{array}$ & $\begin{array}{c}\operatorname{male} \\
0 \\
0 \\
-- \\
0 \\
-- \\
\end{array}$ & $\begin{array}{c}\text { female } \\
0 \\
0 \\
-- \\
0 \\
--\end{array}$ \\
\hline $\begin{array}{l}1990-1991 \\
\text { Pond A } \\
\text { Pond B } \\
\text { Pond C } \\
\text { Pond D } \\
\text { Sun Bay }\end{array}$ & $\begin{array}{c}\operatorname{male} \\
0 \\
0 \\
-- \\
0 \\
--\end{array}$ & $\begin{array}{c}\text { female } \\
0 \\
0 \\
- \\
0 \\
--\end{array}$ & $\begin{array}{c}\text { male } \\
0 \\
0 \\
-- \\
0 \\
--\end{array}$ & $\begin{array}{c}\text { female } \\
0 \\
0 \\
-- \\
0 \\
--\end{array}$ & $\begin{array}{c}\operatorname{male} \\
0 \\
0 \\
-- \\
0 \\
--\end{array}$ & $\begin{array}{c}\text { female } \\
0 \\
0 \\
-- \\
0 \\
--\end{array}$ \\
\hline
\end{tabular}


TABLE III-3. PART IE.

\begin{tabular}{|c|c|c|c|c|c|c|}
\hline & \multicolumn{2}{|c|}{ Hyla squirella } & \multicolumn{2}{|c|}{ Hyla versicolor } & \multicolumn{2}{|c|}{ Bufo quercicus } \\
\hline $\begin{array}{l}1982-1983 \\
\text { Pond A } \\
\text { Pond B } \\
\text { Pond C } \\
\text { Pond D } \\
\text { Sun Bay }\end{array}$ & $\begin{array}{c}\operatorname{male} \\
0 \\
0 \\
0 \\
0 \\
0\end{array}$ & $\begin{array}{c}\text { female } \\
0 \\
0 \\
0 \\
0 \\
0\end{array}$ & $\begin{array}{c}\text { male } \\
0 \\
0 \\
0 \\
0 \\
0\end{array}$ & $\begin{array}{c}\text { female } \\
0 \\
0 \\
0 \\
0 \\
0\end{array}$ & $\begin{array}{c}\operatorname{male} \\
0 \\
0 \\
0 \\
0 \\
0\end{array}$ & $\begin{array}{c}\text { female } \\
0 \\
0 \\
0 \\
0 \\
0\end{array}$ \\
\hline $\begin{array}{l}\text { 1983-1984 } \\
\text { Pond A } \\
\text { Pond B } \\
\text { Pond C } \\
\text { Pond D } \\
\text { Sun Bay }\end{array}$ & $\begin{array}{c}\text { male } \\
0 \\
0 \\
0 \\
0 \\
0\end{array}$ & $\begin{array}{c}\text { female } \\
0 \\
0 \\
0 \\
0 \\
0 \\
\end{array}$ & $\begin{array}{c}\text { male } \\
0 \\
1 \\
0 \\
0 \\
0 \\
\end{array}$ & $\begin{array}{c}\text { female } \\
2 \\
1 \\
0 \\
0 \\
0 \\
\end{array}$ & $\begin{array}{c}\operatorname{male} \\
0 \\
3 \\
0 \\
0 \\
0 \\
\end{array}$ & $\begin{array}{c}\text { female } \\
0 \\
1 \\
0 \\
0 \\
0\end{array}$ \\
\hline $\begin{array}{l}\text { 1984-1985 } \\
\text { Pond A } \\
\text { Pond B } \\
\text { Pond C } \\
\text { Pond D } \\
\text { Sun Bay } \\
\end{array}$ & $\begin{array}{c}\text { male } \\
0 \\
0 \\
0 \\
0 \\
0 \\
\end{array}$ & $\begin{array}{c}\text { female } \\
0 \\
0 \\
0 \\
0 \\
0 \\
\end{array}$ & $\begin{array}{c}\text { male } \\
1 \\
0 \\
0 \\
0 \\
0 \\
\end{array}$ & $\begin{array}{c}\text { female } \\
0 \\
1 \\
0 \\
1 \\
0 \\
\end{array}$ & $\begin{array}{c}\text { male } \\
0 \\
0 \\
0 \\
0 \\
0 \\
\end{array}$ & $\begin{array}{c}\text { female } \\
0 \\
0 \\
0 \\
0 \\
0 \\
\end{array}$ \\
\hline $\begin{array}{l}1985-1986 \\
\text { Pond A } \\
\text { Pond B } \\
\text { Pond C } \\
\text { Pond D } \\
\text { Sun Bay }\end{array}$ & $\begin{array}{c}\operatorname{male} \\
0 \\
0 \\
0 \\
0 \\
0\end{array}$ & $\begin{array}{c}\text { female } \\
0 \\
0 \\
0 \\
0 \\
0\end{array}$ & $\begin{array}{c}\text { male } \\
0 \\
0 \\
0 \\
0 \\
0\end{array}$ & $\begin{array}{c}\text { female } \\
0 \\
1 \\
0 \\
0 \\
0\end{array}$ & $\begin{array}{c}\text { male } \\
0 \\
0 \\
0 \\
0 \\
0\end{array}$ & $\begin{array}{c}\text { female } \\
0 \\
0 \\
0 \\
0 \\
0\end{array}$ \\
\hline $\begin{array}{l}1986-1987 \\
\text { Pond A } \\
\text { Pond B } \\
\text { Pond C } \\
\text { Pond D } \\
\text { Sun Bay } \\
\end{array}$ & $\begin{array}{c}\operatorname{male} \\
0 \\
0 \\
-- \\
0 \\
--\end{array}$ & $\begin{array}{c}\text { female } \\
0 \\
0 \\
-- \\
0 \\
--\end{array}$ & $\begin{array}{c}\text { male } \\
0 \\
0 \\
-- \\
1 \\
--\end{array}$ & $\begin{array}{c}\text { female } \\
0 \\
1 \\
-1 \\
-1 \\
-\end{array}$ & $\begin{array}{c}\operatorname{male} \\
0 \\
0 \\
-- \\
0 \\
--\end{array}$ & $\begin{array}{c}\text { female } \\
0 \\
0 \\
-- \\
0 \\
--\end{array}$ \\
\hline $\begin{array}{l}1987-1988 \\
\text { Pond A } \\
\text { Pond B } \\
\text { Pond C } \\
\text { Pond D } \\
\text { Sun Bay } \\
\end{array}$ & $\begin{array}{c}\operatorname{male} \\
0 \\
0 \\
-- \\
0 \\
--\end{array}$ & $\begin{array}{c}\text { female } \\
0 \\
0 \\
- \\
0 \\
--\end{array}$ & $\begin{array}{c}\text { male } \\
1 \\
0 \\
-- \\
0 \\
--\end{array}$ & $\begin{array}{c}\text { female } \\
0 \\
0 \\
-- \\
0 \\
--\end{array}$ & $\begin{array}{c}\operatorname{male} \\
0 \\
0 \\
-- \\
0 \\
--\end{array}$ & $\begin{array}{c}\text { female } \\
0 \\
0 \\
-- \\
0 \\
--\end{array}$ \\
\hline $\begin{array}{l}1988-1989 \\
\text { Pond A } \\
\text { Pond B } \\
\text { Pond C } \\
\text { Pond D } \\
\text { Sun Bay } \\
\end{array}$ & $\begin{array}{c}\operatorname{male} \\
0 \\
0 \\
-- \\
0 \\
- \\
\end{array}$ & $\begin{array}{c}\text { female } \\
0 \\
0 \\
- \\
0 \\
--\end{array}$ & $\begin{array}{c}\operatorname{male} \\
0 \\
0 \\
-0 \\
0 \\
-- \\
\end{array}$ & $\begin{array}{c}\text { female } \\
0 \\
0 \\
-- \\
0 \\
-- \\
\end{array}$ & $\begin{array}{c}\operatorname{male} \\
0 \\
0 \\
-- \\
0 \\
- \\
\end{array}$ & $\begin{array}{c}\text { female } \\
0 \\
0 \\
- \\
0 \\
-- \\
\end{array}$ \\
\hline $\begin{array}{l}1989-1990 \\
\text { Pond A } \\
\text { Pond B } \\
\text { Pond C } \\
\text { Pond D } \\
\text { Sun Bay } \\
\end{array}$ & $\begin{array}{c}\operatorname{male} \\
0 \\
0 \\
-- \\
0 \\
-- \\
\end{array}$ & $\begin{array}{c}\text { female } \\
0 \\
0 \\
-- \\
0 \\
-- \\
\end{array}$ & $\begin{array}{c}\operatorname{male} \\
0 \\
0 \\
-- \\
0 \\
-- \\
\end{array}$ & $\begin{array}{c}\text { female } \\
0 \\
0 \\
- \\
0 \\
-- \\
\end{array}$ & $\begin{array}{c}\operatorname{male} \\
0 \\
0 \\
-- \\
0 \\
-- \\
\end{array}$ & $\begin{array}{c}\text { female } \\
0 \\
0 \\
- \\
0 \\
-- \\
\end{array}$ \\
\hline $\begin{array}{l}1990-1991 \\
\text { Pond A } \\
\text { Pond B } \\
\text { Pond C } \\
\text { Pond D } \\
\text { Sun Bay } \\
\end{array}$ & $\begin{array}{c}\text { male } \\
0 \\
0 \\
-- \\
0 \\
--\end{array}$ & $\begin{array}{c}\text { female } \\
0 \\
0 \\
-- \\
0 \\
--\end{array}$ & $\begin{array}{c}\operatorname{male} \\
0 \\
1 \\
-1 \\
0 \\
--\end{array}$ & $\begin{array}{c}\text { Eemale } \\
0 \\
0 \\
-- \\
0 \\
- \\
\end{array}$ & $\begin{array}{c}\operatorname{male} \\
0 \\
0 \\
-- \\
0 \\
-- \\
\end{array}$ & $\begin{array}{c}\text { female } \\
0 \\
0 \\
- \\
0 \\
-\end{array}$ \\
\hline
\end{tabular}


TABLE III-3. PART IF.

\begin{tabular}{|c|c|c|c|c|c|c|}
\hline \multirow[b]{2}{*}{$\begin{array}{l}\text { 1982-1983 } \\
\text { Pond A } \\
\text { Pond B } \\
\text { Pond C } \\
\text { Pond D } \\
\text { Sun Bay }\end{array}$} & \multicolumn{2}{|c|}{$\begin{array}{l}\text { Ambystoma } \\
\text { talpoideum }\end{array}$} & \multicolumn{2}{|c|}{$\begin{array}{l}\text { Ambystoma } \\
\text { opacum }\end{array}$} & \multicolumn{2}{|c|}{$\begin{array}{l}\text { Ambystoma } \\
\text { tigrinum }\end{array}$} \\
\hline & $\begin{array}{c}\operatorname{male} \\
0 \\
0 \\
0 \\
0 \\
0\end{array}$ & $\begin{array}{c}\text { female } \\
0 \\
0 \\
0 \\
0 \\
0\end{array}$ & $\begin{array}{c}\text { male } \\
0 \\
0 \\
0 \\
0 \\
0\end{array}$ & $\begin{array}{c}\text { female } \\
0 \\
0 \\
0 \\
0 \\
0 \\
\end{array}$ & $\begin{array}{c}\text { male } \\
0 \\
0 \\
0 \\
0 \\
0\end{array}$ & $\begin{array}{c}\text { female } \\
0 \\
0 \\
0 \\
0 \\
0 \\
\end{array}$ \\
\hline $\begin{array}{l}1983-1984 \\
\text { Pond A } \\
\text { Pond B } \\
\text { Pond C } \\
\text { Pond D } \\
\text { Sun Bay }\end{array}$ & $\begin{array}{c}\text { male } \\
0 \\
0 \\
4 \\
2 \\
20\end{array}$ & $\begin{array}{c}\text { female } \\
0 \\
0 \\
2 \\
1 \\
14\end{array}$ & $\begin{array}{c}\text { male } \\
0 \\
0 \\
0 \\
0 \\
1\end{array}$ & $\begin{array}{c}\text { female } \\
0 \\
0 \\
0 \\
0 \\
0\end{array}$ & $\begin{array}{c}\text { male } \\
0 \\
0 \\
0 \\
0 \\
0\end{array}$ & $\begin{array}{c}\text { female } \\
0 \\
0 \\
0 \\
0 \\
0\end{array}$ \\
\hline $\begin{array}{l}1984-1985 \\
\text { Pond A } \\
\text { Pond B } \\
\text { Pond C } \\
\text { Pond D } \\
\text { Sun Bay }\end{array}$ & $\begin{array}{c}\text { male } \\
0 \\
1 \\
0 \\
0 \\
0 \\
\end{array}$ & $\begin{array}{c}\text { female } \\
0 \\
0 \\
2 \\
0 \\
1 \\
\end{array}$ & $\begin{array}{l}\text { male } \\
0 \\
0 \\
0 \\
0 \\
0\end{array}$ & $\begin{array}{c}\text { female } \\
0 \\
0 \\
0 \\
0 \\
0 \\
\end{array}$ & $\begin{array}{c}\text { male } \\
0 \\
0 \\
0 \\
0 \\
0 \\
\end{array}$ & $\begin{array}{c}\text { female } \\
0 \\
0 \\
0 \\
0 \\
0 \\
\end{array}$ \\
\hline $\begin{array}{l}1985-1986 \\
\text { Pond A } \\
\text { Pond B } \\
\text { Pond C } \\
\text { Pond D } \\
\text { Sun Bay } \\
\end{array}$ & $\begin{array}{c}\text { male } \\
5 \\
6 \\
0 \\
23 \\
4 \\
\end{array}$ & $\begin{array}{c}\text { female } \\
3 \\
3 \\
0 \\
22 \\
5 \\
\end{array}$ & $\begin{array}{c}\text { male } \\
0 \\
0 \\
0 \\
0 \\
0 \\
\end{array}$ & $\begin{array}{c}\text { female } \\
0 \\
0 \\
0 \\
0 \\
0 \\
\end{array}$ & $\begin{array}{c}\text { male } \\
0 \\
0 \\
0 \\
0 \\
0 \\
\end{array}$ & $\begin{array}{c}\text { female } \\
0 \\
1 \\
0 \\
0 \\
0 \\
\end{array}$ \\
\hline $\begin{array}{l}1986-1987 \\
\text { Pond A } \\
\text { Pond B } \\
\text { Pond C } \\
\text { Pond D } \\
\text { Sun Bay }\end{array}$ & $\begin{array}{c}\operatorname{male} \\
5 \\
7 \\
-- \\
21 \\
--\end{array}$ & $\begin{array}{c}\text { female } \\
0 \\
4 \\
-14 \\
--\end{array}$ & $\begin{array}{c}\text { male } \\
0 \\
0 \\
-0 \\
0 \\
--\end{array}$ & $\begin{array}{c}\text { female } \\
0 \\
0 \\
-0 \\
0 \\
--\end{array}$ & $\begin{array}{c}\operatorname{male} \\
0 \\
1 \\
-- \\
0 \\
--\end{array}$ & $\begin{array}{c}\text { female } \\
0 \\
0 \\
-0 \\
0 \\
--\end{array}$ \\
\hline $\begin{array}{l}1987-1988 \\
\text { Pond A } \\
\text { Pond B } \\
\text { Pond C } \\
\text { Pond D } \\
\text { Sun Bay }\end{array}$ & $\begin{array}{c}\operatorname{male} \\
0 \\
6 \\
-- \\
14 \\
--\end{array}$ & $\begin{array}{c}\text { female } \\
1 \\
3 \\
-9 \\
--\end{array}$ & $\begin{array}{c}\operatorname{male} \\
0 \\
0 \\
-- \\
0 \\
--\end{array}$ & $\begin{array}{c}\text { female } \\
0 \\
0 \\
- \\
0 \\
--\end{array}$ & $\begin{array}{c}\operatorname{male}_{0} \\
0 \\
-- \\
0 \\
--\end{array}$ & $\begin{array}{c}\text { female } \\
0 \\
0 \\
- \\
0 \\
--\end{array}$ \\
\hline $\begin{array}{l}1988-1989 \\
\text { Pond A } \\
\text { Pond B } \\
\text { Pond C } \\
\text { Pond D } \\
\text { Sun Bay } \\
\end{array}$ & $\begin{array}{c}\text { male } \\
1 \\
18 \\
-- \\
31 \\
-- \\
\end{array}$ & $\begin{array}{c}\text { female } \\
0 \\
11 \\
-2 \\
20 \\
--\end{array}$ & $\begin{array}{c}\text { male } \\
0 \\
0 \\
-- \\
0 \\
-- \\
\end{array}$ & $\begin{array}{c}\text { female } \\
0 \\
0 \\
- \\
0 \\
-- \\
\end{array}$ & $\begin{array}{c}\operatorname{male} \\
0 \\
1 \\
-- \\
0 \\
-- \\
\end{array}$ & $\begin{array}{c}\text { female } \\
0 \\
0 \\
- \\
0 \\
-- \\
\end{array}$ \\
\hline $\begin{array}{l}1989-1990 \\
\text { Pond A } \\
\text { Pond B } \\
\text { Pond C } \\
\text { Pond D } \\
\text { Sun Bay }\end{array}$ & $\begin{array}{l}\operatorname{male} \\
1 \\
32 \\
-71 \\
--\end{array}$ & $\begin{array}{l}\text { female } \\
\frac{1}{31} \\
-67 \\
--\end{array}$ & $\begin{array}{c}\text { male } \\
0 \\
0 \\
-- \\
0 \\
--\end{array}$ & $\begin{array}{c}\text { female } \\
0 \\
0 \\
-- \\
0 \\
--\end{array}$ & $\begin{array}{c}\text { male } \\
0 \\
1 \\
-0 \\
--\end{array}$ & $\begin{array}{c}\text { female } \\
0 \\
0 \\
-- \\
0 \\
--\end{array}$ \\
\hline $\begin{array}{l}1990-1991 \\
\text { Pond A } \\
\text { Pond B } \\
\text { Pond C } \\
\text { Pond D } \\
\text { Sun Bay }\end{array}$ & $\begin{array}{l}\text { male } \\
1 \\
19 \\
-- \\
59 \\
--\end{array}$ & $\begin{array}{c}\text { female } \\
0 \\
21 \\
-- \\
65 \\
--\end{array}$ & $\begin{array}{c}\text { male } \\
0 \\
0 \\
-- \\
0 \\
--\end{array}$ & $\begin{array}{c}\text { female } \\
0 \\
0 \\
-- \\
0 \\
-\end{array}$ & $\begin{array}{c}\text { male } \\
0 \\
0 \\
-0 \\
--\end{array}$ & $\begin{array}{c}\text { female } \\
0 \\
0 \\
- \\
0 \\
--\end{array}$ \\
\hline
\end{tabular}


TABIE III-3. PART IG.

\begin{tabular}{|c|c|c|c|c|}
\hline & \multicolumn{2}{|c|}{$\begin{array}{l}\text { Acris } \\
\text { grylius }\end{array}$} & \multicolumn{2}{|c|}{$\begin{array}{l}\text { Eurycea } \\
\text { quadridigitata }\end{array}$} \\
\hline $\begin{array}{l}\text { 1982-1983 } \\
\text { Pond A } \\
\text { Pond B } \\
\text { Pond C } \\
\text { Pond D } \\
\text { Sun Bay } \\
\end{array}$ & $\begin{array}{c}\text { male } \\
0 \\
0 \\
0 \\
0 \\
0\end{array}$ & $\begin{array}{c}\text { female } \\
0 \\
0 \\
0 \\
0 \\
0\end{array}$ & $\begin{array}{c}\text { male } \\
0 \\
0 \\
0 \\
0 \\
0 \\
\end{array}$ & $\begin{array}{c}\text { female } \\
0 \\
0 \\
0 \\
0 \\
0\end{array}$ \\
\hline $\begin{array}{l}1983-1984 \\
\text { Pond A } \\
\text { Pond B } \\
\text { Pond C } \\
\text { Pond D } \\
\text { Sun Bay }\end{array}$ & $\begin{array}{c}\text { male } \\
0 \\
0 \\
0 \\
0 \\
0\end{array}$ & $\begin{array}{c}\text { female } \\
0 \\
0 \\
0 \\
0 \\
0\end{array}$ & $\begin{array}{c}\text { male } \\
1 \\
0 \\
0 \\
0 \\
0\end{array}$ & $\begin{array}{c}\text { female } \\
0 \\
0 \\
0 \\
0 \\
0\end{array}$ \\
\hline $\begin{array}{l}\text { 1984-1985 } \\
\text { Pond A } \\
\text { Pond B } \\
\text { Pond C } \\
\text { Pond D } \\
\text { Sun Bay }\end{array}$ & $\begin{array}{c}\text { male } \\
0 \\
0 \\
0 \\
0 \\
0\end{array}$ & $\begin{array}{c}\text { female } \\
0 \\
0 \\
0 \\
0 \\
0\end{array}$ & $\begin{array}{c}\text { male } \\
0 \\
0 \\
0 \\
1 \\
0\end{array}$ & $\begin{array}{c}\text { female } \\
0 \\
0 \\
0 \\
0 \\
0\end{array}$ \\
\hline $\begin{array}{l}\text { 1985-1986 } \\
\text { Pond A } \\
\text { Pond B } \\
\text { Pond C } \\
\text { Pond D } \\
\text { Sun Bay }\end{array}$ & $\begin{array}{c}\text { male } \\
0 \\
0 \\
0 \\
0 \\
0\end{array}$ & $\begin{array}{c}\text { female } \\
0 \\
0 \\
0 \\
0 \\
0\end{array}$ & $\begin{array}{c}\text { male } \\
0 \\
0 \\
0 \\
0 \\
0 \\
\end{array}$ & $\begin{array}{c}\text { female } \\
0 \\
0 \\
0 \\
0 \\
0\end{array}$ \\
\hline $\begin{array}{l}\text { 1986-1987 } \\
\text { Pond A } \\
\text { Pond B } \\
\text { Pond C } \\
\text { Pond D } \\
\text { Sun Bay }\end{array}$ & $\begin{array}{c}\operatorname{male} \\
1 \\
0 \\
-- \\
1 \\
--\end{array}$ & $\begin{array}{c}\text { female } \\
1 \\
0 \\
-- \\
3 \\
--\end{array}$ & $\begin{array}{c}\operatorname{male} \\
0 \\
0 \\
- \\
0 \\
--\end{array}$ & $\begin{array}{c}\text { female } \\
0 \\
0 \\
-- \\
0 \\
--\end{array}$ \\
\hline $\begin{array}{l}1987-1988 \\
\text { Pond A } \\
\text { Pond B } \\
\text { Pond C } \\
\text { Pond D } \\
\text { Sun Bay }\end{array}$ & $\begin{array}{c}\text { male } \\
1 \\
0 \\
-- \\
0 \\
--\end{array}$ & $\begin{array}{c}\text { female } \\
0 \\
0 \\
-0 \\
-\end{array}$ & $\begin{array}{c}\text { male } \\
0 \\
0 \\
-- \\
0 \\
--\end{array}$ & $\begin{array}{c}\text { female } \\
0 \\
0 \\
-- \\
0 \\
--\end{array}$ \\
\hline $\begin{array}{l}\text { 1988-1989 } \\
\text { Pond A } \\
\text { Pond B } \\
\text { Pond C } \\
\text { Pond D } \\
\text { Sun Bay }\end{array}$ & $\begin{array}{c}\text { male } \\
0 \\
0 \\
-- \\
0 \\
--\end{array}$ & $\begin{array}{c}\text { female } \\
0 \\
1 \\
-\frac{1}{6} \\
--\end{array}$ & $\begin{array}{c}\operatorname{male} \\
0 \\
0 \\
-- \\
0 \\
--\end{array}$ & $\begin{array}{c}\text { female } \\
0 \\
0 \\
-- \\
0 \\
-=\end{array}$ \\
\hline $\begin{array}{l}1989-1990 \\
\text { Pond A } \\
\text { Pond B } \\
\text { Pond C } \\
\text { Pond D } \\
\text { Sun Bay }\end{array}$ & $\begin{array}{c}\text { male } \\
0 \\
1 \\
-- \\
9 \\
--\end{array}$ & $\begin{array}{c}\text { female } \\
0 \\
1 \\
-5 \\
-\end{array}$ & $\begin{array}{c}\text { male } \\
0 \\
0 \\
-- \\
0 \\
--\end{array}$ & $\begin{array}{c}\text { female } \\
0 \\
0 \\
-- \\
0 \\
-\end{array}$ \\
\hline $\begin{array}{l}1990-1991 \\
\text { Pond A } \\
\text { Pond B } \\
\text { Pond C } \\
\text { Pond D } \\
\text { Sun Bay }\end{array}$ & $\begin{array}{c}\text { male } \\
0 \\
1 \\
-- \\
8 \\
--\end{array}$ & $\begin{array}{c}\text { female } \\
0 \\
1 \\
-- \\
14 \\
--\end{array}$ & $\begin{array}{c}\text { male } \\
0 \\
0 \\
-- \\
0 \\
--\end{array}$ & $\begin{array}{c}\text { female } \\
0 \\
0 \\
- \\
0 \\
--\end{array}$ \\
\hline
\end{tabular}


TABLE III-3. PART IH. Efts and Adults of Notophthalmus viridescens (breeding populations). "U" indicates unsexable.

\begin{tabular}{|c|c|c|c|c|c|}
\hline & \multicolumn{5}{|c|}{$\begin{array}{l}\text { Notophthalmus viridescens } \\
\text { male/female/male eft/female eft/ "u" eft }\end{array}$} \\
\hline $\begin{array}{l}1982-1983 \\
\text { Pond A } \\
\text { Pond B } \\
\text { Pond C } \\
\text { Pond D } \\
\text { Sun Bay } \\
\end{array}$ & $\begin{array}{l}0 \\
0 \\
0 \\
1 \\
0 \\
\end{array}$ & $\begin{array}{l}0 \\
0 \\
0 \\
0 \\
0 \\
\end{array}$ & $\begin{array}{l}0 \\
0 \\
0 \\
0 \\
0\end{array}$ & $\begin{array}{l}0 \\
0 \\
0 \\
0 \\
0\end{array}$ & $\begin{array}{l}0 \\
0 \\
0 \\
0 \\
0 \\
\end{array}$ \\
\hline $\begin{array}{l}1983-1984 \\
\text { Pond A } \\
\text { Pond B } \\
\text { Pond C } \\
\text { Pond D } \\
\text { Sun Bay } \\
\end{array}$ & $\begin{array}{r}0 \\
0 \\
0 \\
0 \\
13 \\
\end{array}$ & $\begin{array}{l}0 \\
0 \\
0 \\
1 \\
5 \\
\end{array}$ & $\begin{array}{l}1 \\
0 \\
0 \\
0 \\
0 \\
\end{array}$ & $\begin{array}{l}0 \\
0 \\
1 \\
0 \\
0\end{array}$ & $\begin{array}{l}0 \\
0 \\
0 \\
0 \\
0 \\
\end{array}$ \\
\hline $\begin{array}{l}1984-1985 \\
\text { Pond A } \\
\text { Pond B } \\
\text { Pond C } \\
\text { Pond D } \\
\text { Sun Bay }\end{array}$ & $\begin{array}{l}0 \\
0 \\
0 \\
0 \\
0\end{array}$ & $\begin{array}{l}0 \\
0 \\
0 \\
0 \\
0\end{array}$ & $\begin{array}{l}0 \\
0 \\
0 \\
0 \\
0\end{array}$ & $\begin{array}{l}0 \\
0 \\
0 \\
0 \\
0 \\
\end{array}$ & $\begin{array}{l}0 \\
0 \\
0 \\
0 \\
0 \\
\end{array}$ \\
\hline $\begin{array}{l}1985-1986 \\
\text { Pond A } \\
\text { Pond B } \\
\text { Pond C } \\
\text { Pond D } \\
\text { Sun Bay }\end{array}$ & $\begin{array}{l}0 \\
0 \\
0 \\
0 \\
0\end{array}$ & $\begin{array}{l}2 \\
1 \\
0 \\
0 \\
0\end{array}$ & $\begin{array}{l}0 \\
0 \\
0 \\
2 \\
0\end{array}$ & $\begin{array}{l}2 \\
1 \\
0 \\
3 \\
0\end{array}$ & $\begin{array}{l}0 \\
0 \\
0 \\
0 \\
0\end{array}$ \\
\hline $\begin{array}{l}1986-1987 \\
\text { Pond A } \\
\text { Pond B } \\
\text { Pond C } \\
\text { Pond D } \\
\text { Sun Bay }\end{array}$ & $\begin{array}{r}1 \\
0 \\
-1 \\
0\end{array}$ & $\begin{array}{r}2 \\
1 \\
-- \\
3 \\
0\end{array}$ & $\begin{array}{r}0 \\
0 \\
-0 \\
0 \\
0\end{array}$ & $\begin{array}{r}0 \\
0 \\
-- \\
1 \\
0\end{array}$ & $\begin{array}{r}0 \\
0 \\
-1 \\
0 \\
0\end{array}$ \\
\hline $\begin{array}{l}1987-1988 \\
\text { Pond A } \\
\text { Pond B } \\
\text { Pond C } \\
\text { Pond D } \\
\text { Sun Bay }\end{array}$ & $\begin{array}{r}3 \\
0 \\
-0 \\
--\end{array}$ & $\begin{array}{r}8 \\
1 \\
-0 \\
--\end{array}$ & $\begin{array}{r}0 \\
0 \\
-0 \\
--\end{array}$ & $\begin{array}{r}0 \\
1 \\
-1 \\
-1 \\
-\end{array}$ & $\begin{array}{r}3 \\
1 \\
-1 \\
0 \\
--\end{array}$ \\
\hline $\begin{array}{l}1988-1989 \\
\text { Pond A } \\
\text { Pond B } \\
\text { Pond C } \\
\text { Pond D } \\
\text { Sun Bay }\end{array}$ & $\begin{array}{r}1 \\
0 \\
-1 \\
--\end{array}$ & $\begin{array}{r}1 \\
0 \\
-2 \\
--\end{array}$ & $\begin{array}{r}1 \\
0 \\
-0 \\
--\end{array}$ & $\begin{array}{r}0 \\
0 \\
-0 \\
--\end{array}$ & $\begin{array}{r}0 \\
0 \\
-2 \\
-2\end{array}$ \\
\hline
\end{tabular}




\begin{tabular}{||r|rrrrr||}
\hline $\begin{array}{l}1989-1990 \\
\text { Pond A }\end{array}$ & 0 & 0 & 1 & 1 & 0 \\
Pond B & 2 & 0 & 0 & 1 & 0 \\
Pond C & -- & -- & -- & -- & -- \\
Pond D & 14 & 14 & 6 & 8 & -1 \\
Sun Bay & -- & -- & -- & - & \\
\hline $1990-1991$ & & & & & 1 \\
Pond A & 1 & 0 & 1 & 2 & 0 \\
Pond B & 0 & 0 & 0 & 0 & -- \\
Pond C & -- & -- & -- & -- & 1 \\
Pond D & 5 & 5 & 10 & 17 & -1 \\
Sun Bay & -- & -- & -- & -- & \\
\hline
\end{tabular}

TABLE III-3. PART IIA. Gilled, probably paedomorphic Notophthalmus viridescens caught in minnow traps and subset later recaptured at the drift fence.

\begin{tabular}{||c|ccc||}
\hline & \multicolumn{3}{|c|}{ Minnow Trap Captures } \\
\hline $1986-1987$ & male & female & recaptures \\
Pond A & 7 & 11 & 0 \\
Pond B & 0 & 0 & 0 \\
Pond D & 8 & 14 & 0 \\
\hline
\end{tabular}

Gilled, probably paedomorphic Ambystoma talpoideum caught in minnow traps and subset later recaptured at the drift fence.

\begin{tabular}{|c|cccc||}
\hline & \multicolumn{4}{|c|}{ Minnow } \\
\hline & Trap Captures \\
\hline $1986-1987$ & male & female & Mrecap & Frecap \\
Pond A & 0 & 1 & 0 & 0 \\
Pond B & 12 & 15 & 4 & 3 \\
Pond D & 5 & 7 & 4 & 3 \\
\hline
\end{tabular}


Ambystoma talpoideum juveniles mid-May through 31 December. Sexes have been pooled.

\begin{tabular}{|c|r|}
\hline 1986 & \\
Pond A & 5 \\
Pond B & 32 \\
Pond D & 5 \\
\hline 1987 & \\
Pond A & 1 \\
Pond B & 21 \\
Pond D & 196 \\
\hline 1988 & \\
Pond A & 0 \\
Pond B & 69 \\
Pond D & 95 \\
\hline 1989 & \\
Pond A & 0 \\
Pond B & 34 \\
Pond D & 82 \\
\hline 1990 & \\
Pond A & 0 \\
Pond B & 39 \\
Pond D & 5 \\
\hline 1991 & \\
Pond A & 0 \\
Pond B & 78 \\
Pond D & 176 \\
\hline
\end{tabular}

Ex-paedomorphic Ambystoma talpoideum and juveniles that overwintered. The sexes have been pooled.

\begin{tabular}{|c|r|}
\hline $1986-1987$ & \\
Pond A & 3 \\
Pond B & 68 \\
Pond D & 30 \\
\hline $1987-1988$ & \\
Pond A & 0 \\
Pond B & 0 \\
Pond D & 116 \\
\hline $1988-1989$ & \\
Pond A & 0 \\
Pond B & 76 \\
Pond D & 43 \\
\hline $1989-1990$ & \\
Pond A & 0 \\
Pond B & 55 \\
Pond D & 121 \\
\hline 1990-1991 & \\
Pond A & 0 \\
Pond B & 64 \\
Pond D & 20 \\
\hline
\end{tabular}


Juvenile Ambystoma talpoideum Produced at the Refuge Ponda. This table includes juveniles that overwintered and ex-paedomorphs, except for 1991 when these were not sampled.

\begin{tabular}{|c|r|r|r|r|r|}
\hline & Pond A & Pond B & Pond C & Pond D & Totals \\
\hline 1984 & 0 & 0 & 0 & 0 & 0 \\
\hline 1985 & 0 & 0 & 0 & 0 & 0 \\
\hline 1986 & 9 & 100 & -- & 35 & 144 \\
\hline 1987 & 1 & 21 & -- & 312 & 334 \\
\hline 1988 & 0 & 145 & -- & 138 & 283 \\
\hline 1989 & 0 & 89 & -- & 203 & 292 \\
\hline 1990 & 0 & 103 & -- & 25 & 128 \\
\hline 1991 & 0 & 78 & -- & 176 & 254 \\
\hline Totals & 10 & 536 & 0 & 889 & 1435 \\
\hline
\end{tabular}

TABLE III-3. PART IIB. Juvenile Notopthalmus viridescens produced at the Refuge Ponds.

\begin{tabular}{|c|r|r|r|r|r|}
\hline & Pond A & Pond B & Pond C & Pond D & Totals \\
\hline 1984 & 0 & 0 & 0 & 0 & 0 \\
\hline 1985 & 0 & 0 & 0 & 0 & 0 \\
\hline 1986 & 11 & 0 & -- & 8 & 19 \\
\hline 1987 & 41 & 2 & -- & 1 & 44 \\
\hline 1988 & 88 & 0 & -- & 58 & 146 \\
\hline 1989 & 329 & 0 & -- & 45 & 374 \\
\hline 1990 & 56 & 0 & -- & 67 & 123 \\
\hline 1991 & 183 & 0 & -- & 7 & 190 \\
\hline Totals & 708 & 2 & 0 & 186 & 896 \\
\hline
\end{tabular}


TABLE III-3. PART IIC. Juvenile Bufo terrestris produced at the Refuge Ponds.

\begin{tabular}{|r|r|r|r|r|r|}
\hline & Pond A & Pond B & Pond C & Pond D & Totals \\
\hline 1984 & 0 & 0 & 0 & 0 & 0 \\
\hline 1985 & 50 & 16 & 0 & 0 & 66 \\
\hline 1986 & 1 & 1 & -- & 14 & 16 \\
\hline 1987 & 0 & 0 & -- & 0 & 0 \\
\hline 1988 & 3 & 0 & -- & 298 & 301 \\
\hline 1989 & 0 & 0 & -- & 210 & 210 \\
\hline 1990 & 2 & 0 & -- & 19 & 21 \\
\hline 1991 & 2 & 0 & -- & 144 & 146 \\
\hline Totals & 58 & 17 & 0 & 685 & 760 \\
\hline
\end{tabular}

TABLE III-3. PART IID. Juvenile Acris gryllus produced at the Refuge Ponds.

\begin{tabular}{|c|r|r|r|r|r|}
\hline & Pond A & Pond B & Pond C & Pond D & Totals \\
\hline 1984 & 0 & 0 & 0 & 0 & 0 \\
\hline 1985 & 0 & 0 & 0 & 0 & 0 \\
\hline 1986 & 0 & 0 & -- & 0 & 0 \\
\hline 1987 & 5 & 0 & -- & 13 & 18 \\
\hline 1988 & 0 & 0 & -- & 31 & 31 \\
\hline 1989 & 1 & 15 & -- & 54 & 70 \\
\hline 1990 & 0 & 6 & -- & 49 & 55 \\
\hline 1991 & 3 & 10 & -- & 207 & 220 \\
\hline Totals & 9 & 31 & 0 & 354 & 394 \\
\hline
\end{tabular}


TABLE III-3. PART IIE. Juvenile Pseudacris crucifer produced at the Refuge Ponds.

\begin{tabular}{|r|r|r|r|r|r|}
\hline & Pond A & Pond B & Pond C & Pond D & Totals \\
\hline 1984 & 0 & 0 & 0 & 0 & 0 \\
\hline 1985 & 306 & 85 & 313 & 640 & 1344 \\
\hline 1986 & 0 & 3 & -- & 1 & 4 \\
\hline 1987 & 0 & 0 & -- & 1 & 1 \\
\hline 1988 & 97 & 147 & -- & 8 & 252 \\
\hline 1989 & 0 & 6 & -- & 3 & 9 \\
\hline 1990 & 1 & 0 & -- & 54 & 55 \\
\hline 1991 & 0 & 2 & -- & 53 & 55 \\
\hline Totals & 404 & 243 & 313 & 760 & 1720 \\
\hline
\end{tabular}

TABLE III-3. PART IIF. Juvenile Hyla gratiosa produced at the Refuge Ponds.

\begin{tabular}{|c|r|r|r|r|r|}
\hline & Pond A & Pond B & Pond C & Pond D & Totals \\
\hline 1984 & 0 & 0 & 0 & 0 & 0 \\
\hline 1985 & 24 & 100 & 0 & 41 & 165 \\
\hline 1986 & 10 & 15 & -- & 98 & 123 \\
\hline 1987 & 32 & 17 & -- & 5 & 54 \\
\hline 1988 & 2 & 16 & -- & 65 & 83 \\
\hline 1989 & 3 & 138 & -- & 23 & 164 \\
\hline 1990 & 6 & 35 & -- & 126 & 167 \\
\hline 1991 & 1 & 69 & -- & 45 & 115 \\
\hline Totals & 78 & 390 & 0 & 403 & 871 \\
\hline
\end{tabular}


TABLE III-3. PART IIG. Juvenile Hyla femoralis produced at the Refuge Ponds.

\begin{tabular}{|c|r|r|r|r|r|}
\hline & Pond A & Pond B & Pond C & Pond D & Totals \\
\hline 1984 & 1 & 0 & 0 & 0 & 1 \\
\hline 1985 & 0 & 0 & 0 & 0 & 0 \\
\hline 1986 & 0 & 0 & -- & 0 & 0 \\
\hline 1987 & 0 & 0 & -- & 0 & 0 \\
\hline 1988 & 0 & 0 & -- & 0 & 0 \\
\hline 1989 & 0 & 0 & -- & 0 & 0 \\
\hline 1990 & 0 & 0 & -- & 0 & 0 \\
\hline 1991 & 0 & 0 & -- & 0 & 0 \\
\hline Totals & 1 & 0 & 0 & 0 & 1 \\
\hline
\end{tabular}

TABLE III-3. PART IIH. Juvenile Hyla chrysoscelis produced at the Refuge Ponds.

\begin{tabular}{|r|r|r|r|r|r|}
\hline & Pond A & Pond B & Pond C & Pond D & Totals \\
\hline 1984 & 7 & 0 & 0 & 0 & 7 \\
\hline 1985 & 59 & 77 & 0 & 6 & 142 \\
\hline 1986 & 0 & 0 & -- & 1 & 1 \\
\hline 1987 & 2 & 2 & -- & 0 & 4 \\
\hline 1988 & 0 & 0 & -- & 1 & 1 \\
\hline 1989 & 0 & 0 & -- & 1 & 1 \\
\hline 1990 & 1 & 0 & -- & 6 & 7 \\
\hline 1991 & 0 & 3 & -- & 0 & 3 \\
\hline Totals & 69 & 82 & 0 & 15 & 166 \\
\hline
\end{tabular}


TABLE III-3. PART IIi. Juvenile Pseudacris ornata produced at the Refuge Ponds.

\begin{tabular}{|c|r|r|r|r|r|}
\hline & Pond A & Pond B & Pond C & Pond D & Totals \\
\hline 1984 & 0 & 0 & 0 & 0 & 0 \\
\hline 1985 & 0 & 0 & 0 & 0 & 0 \\
\hline 1986 & 0 & 0 & -- & 0 & 0 \\
\hline 1987 & 0 & 0 & -- & 0 & 0 \\
\hline 1988 & 1 & 6 & -- & 0 & 7 \\
\hline 1989 & 0 & 1 & -- & 1 & 2 \\
\hline 1990 & 0 & 0 & -- & 0 & 0 \\
\hline 1991 & 0 & 0 & -- & 0 & 0 \\
\hline Totals & 1 & 7 & 0 & 1 & 9 \\
\hline
\end{tabular}

TABLE III-3. PART IIJ. Juvenile Rana catesbeiana produced at the Refuge Ponds.

\begin{tabular}{|c|r|r|r|r|r|}
\hline & Pond A & Pond B & Pond C & Pond D & Totals \\
\hline 1984 & 0 & 0 & 0 & 0 & 0 \\
\hline 1985 & 0 & 0 & 0 & 0 & 0 \\
\hline 1986 & 0 & 0 & -- & 0 & 0 \\
\hline 1987 & 0 & 9 & -- & 0 & 9 \\
\hline 1988 & 0 & 0 & -- & 0 & 0 \\
\hline 1989 & 0 & 0 & -- & 0 & 0 \\
\hline 1990 & 0 & 0 & -- & 0 & 0 \\
\hline 1991 & 6 & 0 & -- & 0 & 6 \\
\hline Totals & 6 & 9 & 0 & 0 & 15 \\
\hline
\end{tabular}


TABLE III-3. PART IIR. Juvenile Rana clamitans produced at the Refuge Ponds.

\begin{tabular}{|c|r|r|r|r|r||}
\hline & Pond A & Pond B & Pond C & Pond D & Totals \\
\hline 1984 & 0 & 0 & 0 & 0 & 0 \\
\hline 1985 & 0 & 0 & 0 & 0 & 0 \\
\hline 1986 & 0 & 0 & -- & 0 & 0 \\
\hline 1987 & 0 & 0 & -- & 0 & 0 \\
\hline 1988 & 0 & 0 & -- & 0 & 0 \\
\hline 1989 & 0 & 0 & - & 0 & 0 \\
\hline 1990 & 0 & 0 & -- & 0 & 0 \\
\hline 1991 & 88 & 0 & -- & 0 & 88 \\
\hline Totals & 88 & 0 & 0 & 0 & 88 \\
\hline
\end{tabular}

TABLE III-3. PART IIL. Juvenile Rana utricularia produced at the Refuge Ponds.

\begin{tabular}{|r|r|r|r|r|r||}
\hline & Pond A & Pond B & Pond C & Pond D & Totals \\
\hline 1984 & 0 & 0 & 0 & 0 & 0 \\
\hline 1985 & 19 & 646 & 0 & 0 & 665 \\
\hline 1986 & 1 & 6 & -- & 7 & 14 \\
\hline 1987 & 21 & 0 & -- & 0 & 21 \\
\hline 1988 & 287 & 72 & -- & 0 & 359 \\
\hline 1989 & 18 & 5 & -- & 0 & 23 \\
\hline 1990 & 76 & 177 & -- & 0 & 253 \\
\hline 1991 & 176 & 3 & -- & 3 & 182 \\
\hline Totals & 598 & 909 & 0 & 10 & 1517 \\
\hline
\end{tabular}




\section{LOCATION OF REFUGE PONDS}

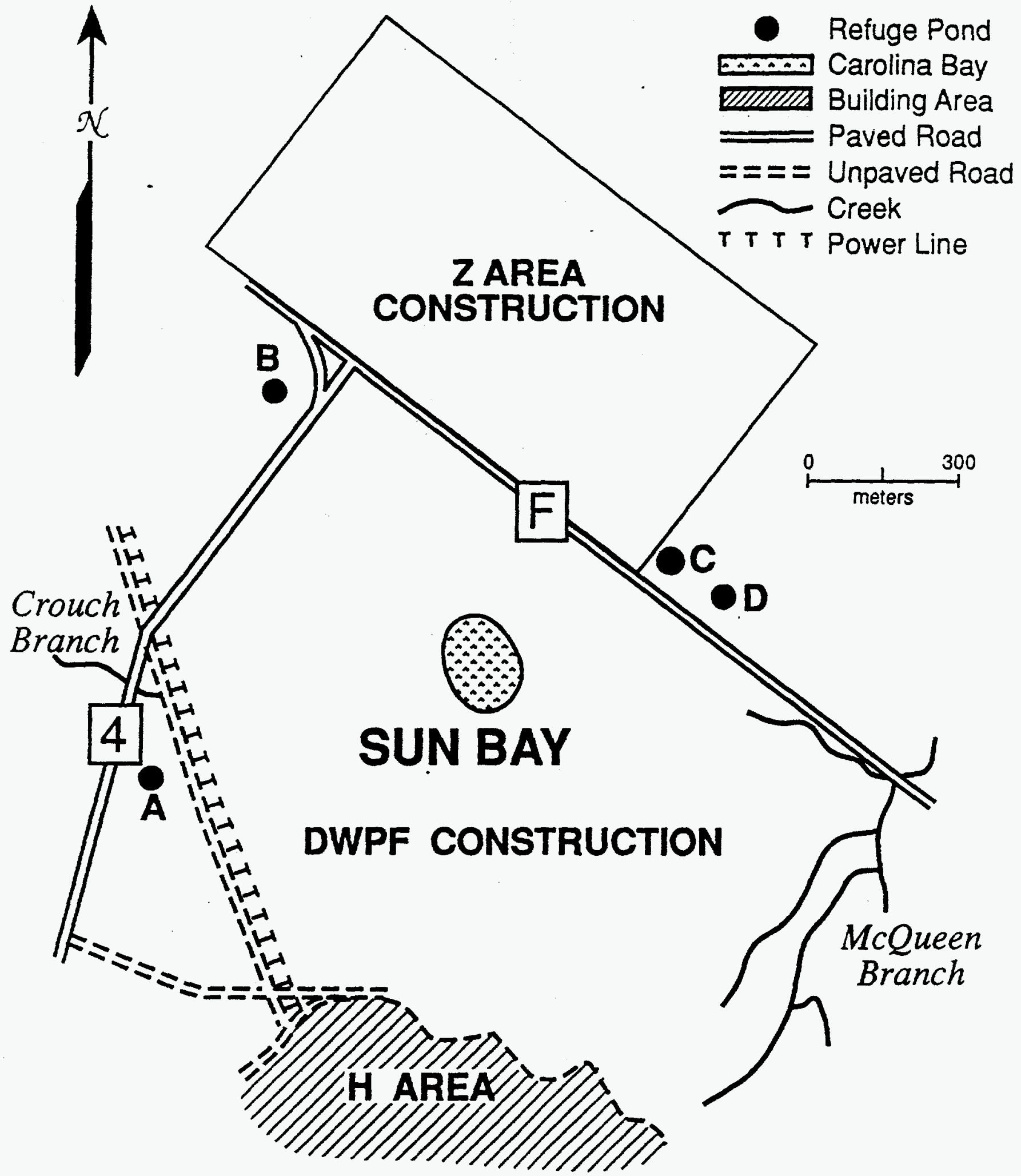

Fig. III-1. Location of refuge ponds in relation to DWPF construction. 


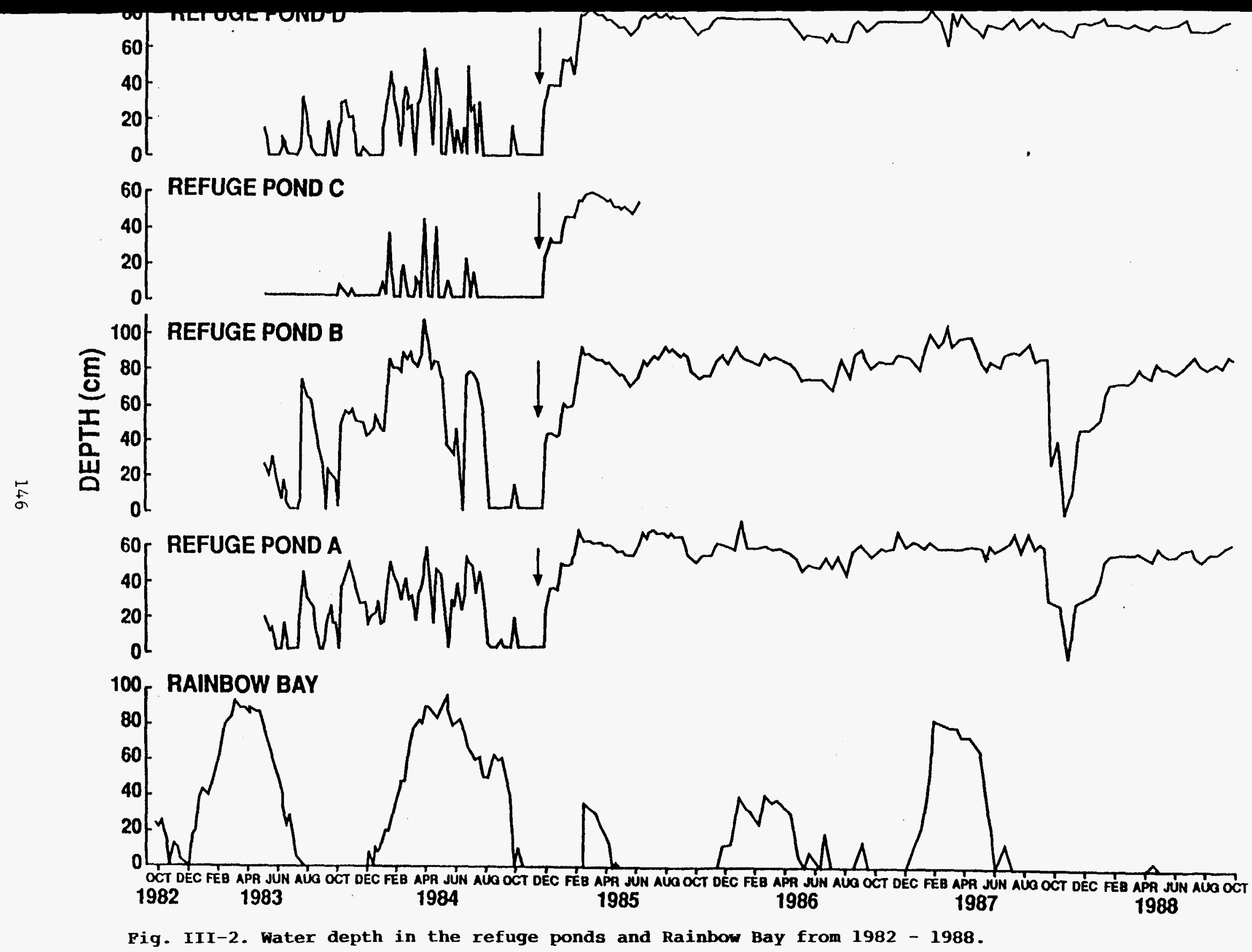




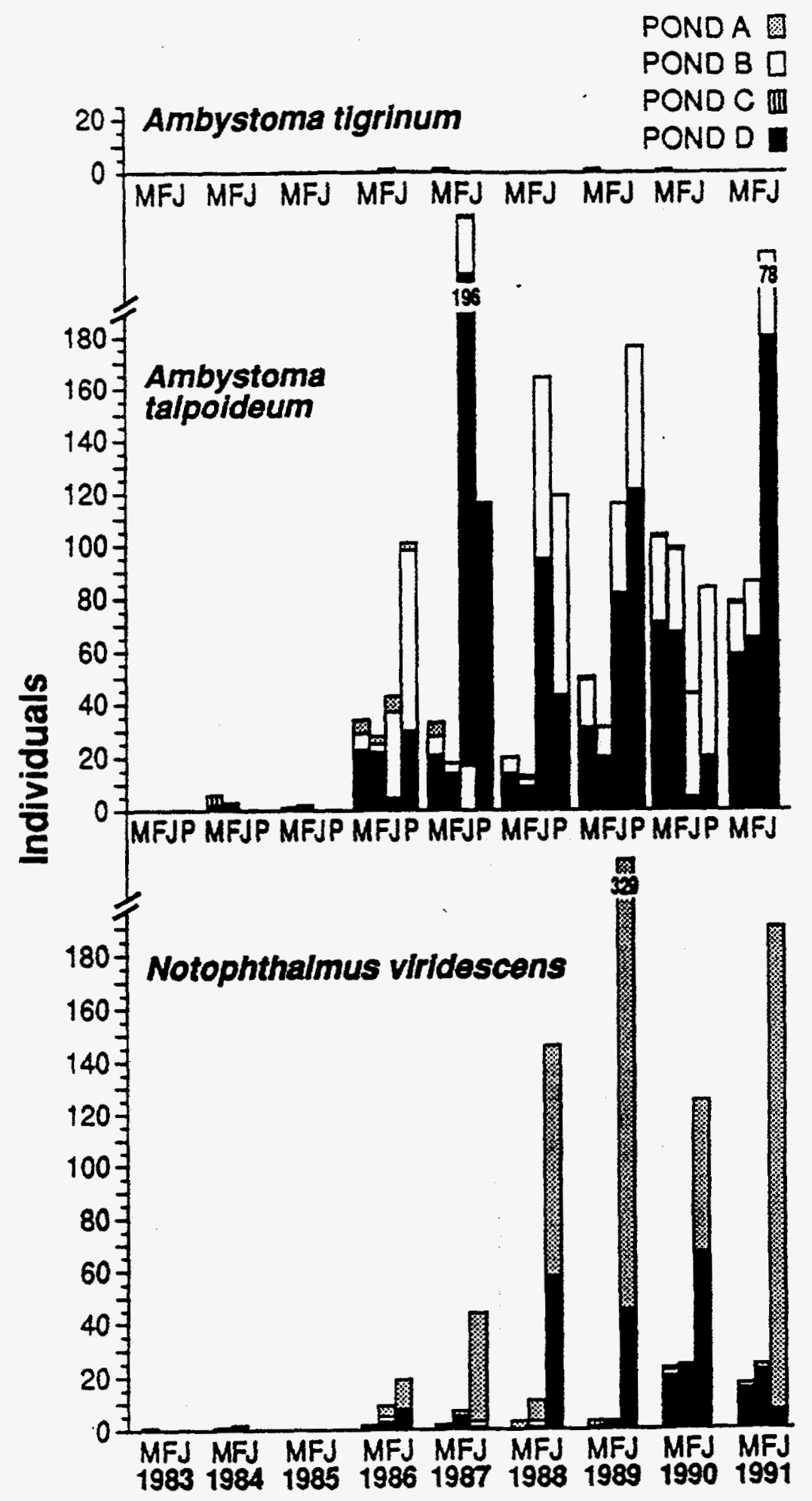

Fig. III-4. Number of breeding adult and metamorphic salamanders in refuge ponds

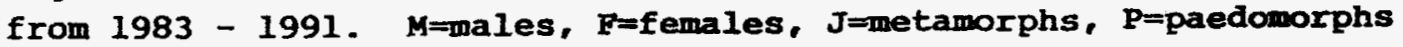




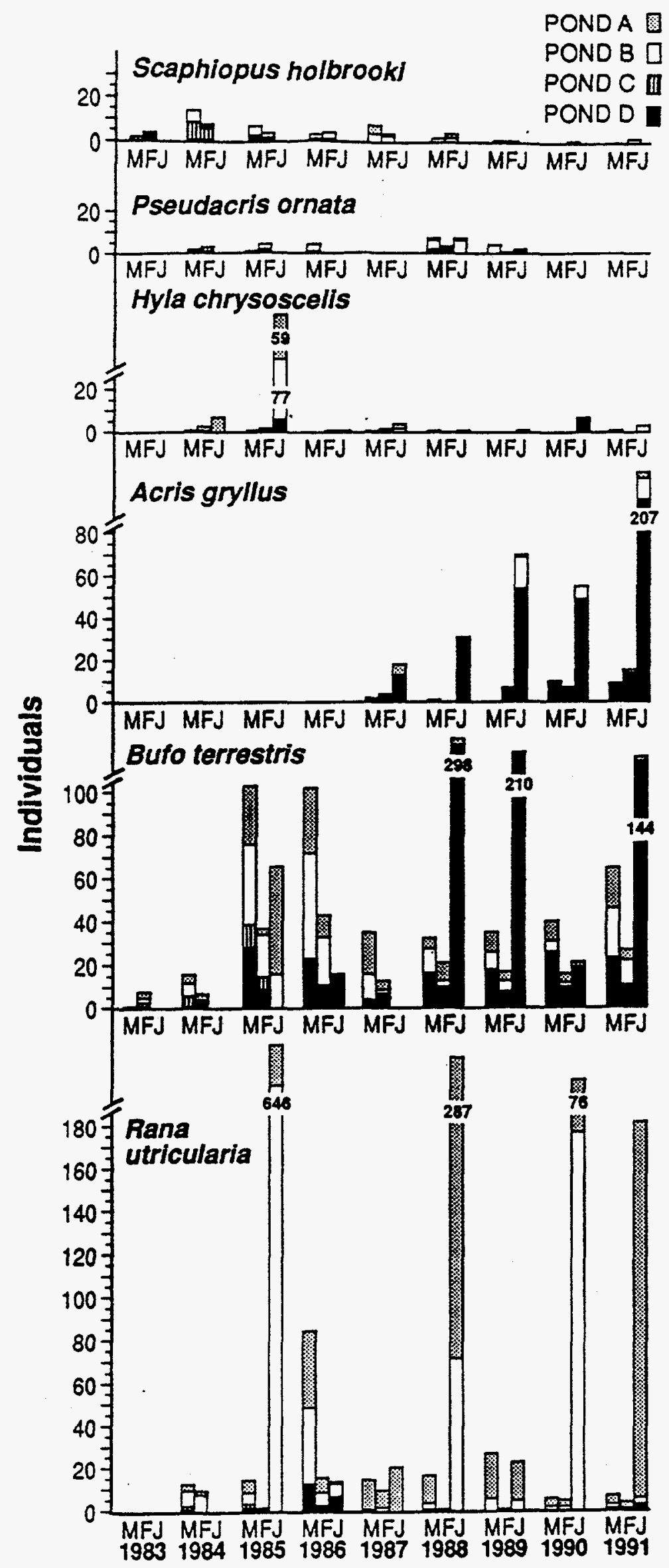

g. III-3. Number of breeding adult and metamorphic anurans in refuge ponds from 83 - 1991. M-males, F=females, J-metamorphs 